\title{
CALCULATING ALPHA EIGENVALUES AND EIGENFUNCTIONS WITH A MARKOV TRANSITION RATE MATRIX MONTE CARLO METHOD
}

\author{
by \\ Benjamin Robert Betzler
A dissertation submitted in partial fulfillment of the requirements for the degree of Doctor of Philosophy (Nuclear Engineering and Radiological Sciences) in the University of Michigan
2014

\section{Doctoral Committee:}

Professor William R. Martin, Chair

Associate Professor Brian Denton

Professor Thomas Downar

Research Scientist Brian Kiedrowski, Los Alamos National Laboratory Professor John C. Lee 
Benjamin R. Betzler, 2014

(C)

All Rights Reserved 
In memory of my brother Michael, who I miss every day 


\section{ACKNOWLEDGEMENTS}

To Professor William Martin, my advisor, for his profound wisdom, guidance, patience, and support,

To Dr. Brian Kiedrowski, my de-facto co-chair, for helping me formulate this thesis topic, and for his invaluable advice,

To Professor Thomas Downar and Professor John C. Lee, for their teachings, mentorship, and input on this thesis,

To Professor Brian Denton, for broadening my knowledge of Markov processes,

To Kaushik Banerjee and Gokhan Yesilyurt, for setting a shining example for any graduate student,

To Eva Sunny and Andrew Pavlou, for their collaborations during the FSV project,

To Tim Burke and Sean Carney, for the time spent in Los Alamos, and for deep discussions,

To my old man, my mother, and my sister Kathy, for their delightfully dysfunctional love,

To Eric Miller and Jacob Faust, for making graduate school fun, and for not kicking me out of the house,

To Andrew Kim and Rachel Lee, for keeping me accountable, and for the occasional dinner,

To all my friends, for being my family,

And to Song, my muse, my love, for everything short of the stars, for her patience, understanding, and unwavering support, without whom I would undoubtedly be somewhere very, very far from here,

thank you. For all these good people, I consider myself one of the luckiest individuals on this green earth. 


\section{TABLE OF CONTENTS}

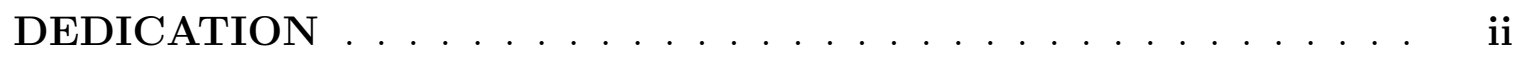

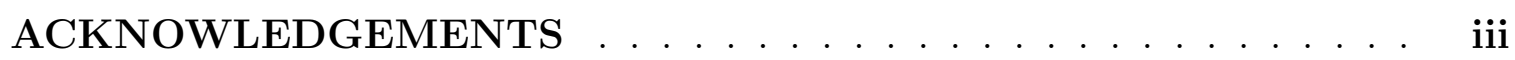

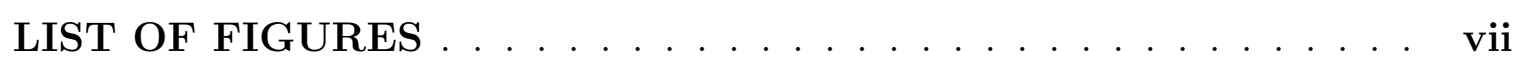

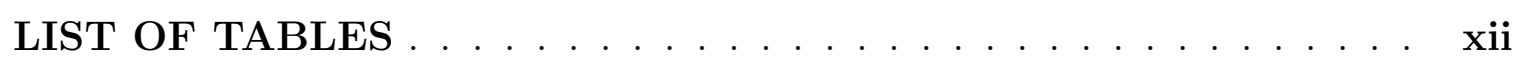

LIST OF ABBREVIATIONS . . . . . . . . . . . . . . xiv

Chapter 1 Introduction . . . . . . . . . . . . . . . . . 1

1.1 Neutron Transport . . . . . . . . . . . . . . . . . 2

1.2 The Monte Carlo Method . . . . . . . . . . . . . . . . 3

1.3 Applications for the Transition Rate Matrix Method . . . . . . . . . 4

1.3.1 Active Nondestructive Assay . . . . . . . . . . . . . . . 5

1.3.2 Pulsed-Neutron Experiments . . . . . . . . . . . . . . . 5

1.3.3 Accelerator-Driven Subcritical Systems . . . . . . . . . . 7

1.3.4 Fast-Burst Reactors . . . . . . . . . . . . . . . 8

1.4 Thesis Objective and Outline . . . . . . . . . . . . . . . 9

References . . . . . . . . . . . . . . . . . . . 10

Chapter 2 Time-Dependent Neutron Transport . . . . . . . . . . . 13

2.1 Time-Dependent Equations . . . . . . . . . . . . . . . . . . . 14

2.2 Solutions to the Neutron Balance Equation . . . . . . . . . . . 16

2.2 .1 Direct Methods . . . . . . . . . . . . . . . . . . . 17

2.2.2 Space-Time Factorization Methods . . . . . . . . . . . 18

2.2 .3 Expansion Methods . . . . . . . . . . . . . . . . . . . . . 21

2.2 .4 Time-Dependent Monte Carlo . . . . . . . . . . . . . . . 22

2.3 The $\alpha$ Eigenvalue . . . . . . . . . . . . . . . . . . . . . . . . 26

2.3.1 The Linear Transport Operator . . . . . . . . . . . . . 27

2.3.2 Relation to Other Eigenvalue Problems . . . . . . . . . . . . 28

2.3 .3 Spectrum . . . . . . . . . . . . . . . . . . 34

2.3.4 Eigenfunction Expansion . . . . . . . . . . . . . . . . . . . 38

2.4 Measuring $\alpha$ Eigenvalues . . . . . . . . . . . . . . . . . . . 43

2.4.1 Pulsed-Neutron Experiments . . . . . . . . . . . . . . . . . 44

2.4 .2 Rossi- $\alpha$ Experiments . . . . . . . . . . . . . . . . . . 47

2.5 Calculating $\alpha$ Eigenvalues $\ldots \ldots \ldots \ldots \ldots \ldots$ 
2.5.1 Fixed-Source Calculations . . . . . . . . . . . . . . . . . . 48

2.5.2 Diffusion Theory Methods . . . . . . . . . . . . . . . . . 51

2.5.3 Transport Theory Methods . . . . . . . . . . . . . 55

2.5.4 Monte Carlo Methods . . . . . . . . . . . . . . . . . . 59

2.5 .5 Summary . . . . . . . . . . . . . . . . . . . 61

References ........................... 63

Chapter 3 The Markov Transition Rate Matrix Method . . . . . . 68

3.1 Theoretical Formulation . . . . . . . . . . . . . . . . . . . . . . 69

3.1.1 Markov Processes . . . . . . . . . . . . . . . . . 69

3.1.2 The Time-Dependent Neutron Importance Equation . . . . . . 70

3.1.3 The Transition Rate Matrix . . . . . . . . . . . . . . . . 73

3.1.4 The Forward and Adjoint $\alpha$-Eigenvalue Problems . . . . . . 79

3.1.5 Corollary to the Fission Matrix . . . . . . . . . . . . . 82

3.2 Derivations of Transition Rate Matrices . . . . . . . . . . . . . . 83

$3.2 .1 \quad$ Infinite Medium . . . . . . . . . . . . . . . . . . . . . 84

3.2 .2 One-Dimensional Media . . . . . . . . . . . . . . . . . . . . 89

3.2 .3 Multi-Dimensional Media . . . . . . . . . . . . . . . 96

3.3 Transition Rate Calculations . . . . . . . . . . . . . . . . . . . 97

3.3.1 Rate Approximations . . . . . . . . . . . . . . . . . . . . 9 98

3.3 .2 Monte Carlo Tallies . . . . . . . . . . . . . . . . . . . . . . 101

3.4 Eigenvalue Algorithms . . . . . . . . . . . . . . . . . . . . . . 104

3.4.1 Eigenvalue-Revealing Factorizations . . . . . . . . . . . . 105

3.4 .2 Iterative Methods . . . . . . . . . . . . . . . . . . 107

References . . . . . . . . . . . . . . . . . . . . . . . 109

Chapter 4 Eigenvalues and Eigenfunctions of Infinite Media . . . . 111

4.1 Code (TORTE) . . . . . . . . . . . . . . . . . . . . . . . 111

4.1.1 Tallying the Transition Rate Matrix . . . . . . . . . . . . . . 112

4.1.2 Eigenvalues and Eigenfunctions . . . . . . . . . . . . . . 113

4.2 Multigroup Verification . . . . . . . . . . . . . . . . . . . 114

4.2 .1 Few-Group Media . . . . . . . . . . . . . . . . . . . . 115

4.2 .2 81-Group Media . . . . . . . . . . . . . . . . . . . . 117

4.3 Continuous-Energy Media . . . . . . . . . . . . . . . . . . . 121

4.3 .1 Eigenvalue Spectrum . . . . . . . . . . . . . . . . . 122

4.3 .2 Energy Intervals . . . . . . . . . . . . . . . . . . . . 125

4.3 .3 Eigenfunctions . . . . . . . . . . . . . . . . . . . . 130

4.3.4 Eigenvalue Convergence . . . . . . . . . . . . . . . . . 133

4.3.5 Comparison to Time-Dependent Monte Carlo . . . . . . . . 138

4.3.6 Quantification of the Residual . . . . . . . . . . . . . . . 141

4.4 Discussion . . . . . . . . . . . . . . . . . . . . . . . . 145

References . . . . . . . . . . . . . . . . . . 145

Chapter 5 Eigenvalues and Eigenfunctions of Slabs $\ldots \ldots \ldots$. . . . 147

5.1 Codes (TORTE-1D and TORTB-1D) . . . . . . . . . . 148 
5.1.1 Tallying the Transition Rate Matrix . . . . . . . . . . . . . . . 149

5.1 .2 Eigenvalues and Eigenfunctions . . . . . . . . . . . . . 150

5.1 .3 Visualization of Flux Solutions . . . . . . . . . . . . . 151

5.2 One-Speed Verification . . . . . . . . . . . . . . . . 151

5.2 .1 Non-Multiplying Media . . . . . . . . . . . . . . . . . . . . 152

5.2 .2 Multiplying Media . . . . . . . . . . . . . . . . . 158

5.2 .3 Mixed Media . . . . . . . . . . . . . . . . 161

5.2.4 Position and Direction Intervals . . . . . . . . . . . . . . 174

5.2 .5 On the Residual . . . . . . . . . . . . . . . . . . . . . 179

5.3 Continuous-Energy Slabs . . . . . . . . . . . . . . . . . 180

5.3.1 Eigenvalue Spectrum . . . . . . . . . . . . . . . . . . . . 182

5.3.2 Discretization of the Phase Space . . . . . . . . . . . . . . . 184

5.3 .3 Shape Eigenfunctions . . . . . . . . . . . . . . . . 186

5.3.4 Flux Transients . . . . . . . . . . . . . . . . . . . . . 189

5.4 Discussion . . . . . . . . . . . . . . . . . . . 191

References ............................. 192

Chapter 6 Three-Dimensional Eigenvalues and Eigenfunctions . . . 194

6.1 Code $($ OpenTRMM) . . . . . . . . . . . . . . . . 195

6.2 The Hydrogenous Cube . . . . . . . . . . . . . . . . . . 196

6.2.1 Eigenvalue Spectrum . . . . . . . . . . . . . . . 196

6.2.2 Shape Eigenfunctions . . . . . . . . . . . . . . . . . . . . 198

6.2.3 Eigenfunction Expansion . . . . . . . . . . . . . . . . . 202

6.3 The Fast Burst Reactor CALIBAN . . . . . . . . . . . . . . . . 205

6.3.1 Eigenvalue Spectrum . . . . . . . . . . . . . . . . 207

6.3.2 Shape Eigenfunctions . . . . . . . . . . . . . . . . . . 211

6.4 The Fort St. Vrain Reactor . . . . . . . . . . . . . . . . . . 215

6.4.1 OpenMC Model . . . . . . . . . . . . . . . . 215

6.4 .2 OpenTRMM Results . . . . . . . . . . . . . . 217

6.5 Discussion . . . . . . . . . . . . . . . . . . . . . 222

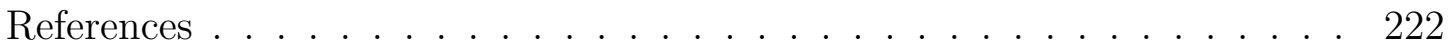

Chapter 7 Summary and Future Work . . . . . . . . . . . . . . . . 224

7.1 Calculated $\alpha$ Eigenvalues and Eigenfunctions . . . . . . . . . . . 225

7.2 Future Work . . . . . . . . . . . . . . . . . . . 226

References ....................... . . 227 


\section{LIST OF FIGURES}

1.1 Measured time-dependent responses of three different detectors for a subcritical control rod configuration of Fort St. Vrain demonstrate the strong spatial dependence of the neutron flux for large systems. . . .

2.1 This idealized illustration of the spectrum of the linear transport operator plotted in the complex plane shows the different features present in the spectrum. . . . . . . . . . . . . . . . .

2.2 The measured detector response to a neutron pulse plotted on the appropriate time scale shows the three characteristic time intervals dominated by the different kinetic modes. . . . . . . . . . . . . .

2.3 MCNP5-calculated prompt detector responses for a pulsed subcritical configuration of FSV yields very few counts in interval III due to the exponentially decaying prompt fundamental mode. . . . . . . . . . .

2.4 MCNP5-calculated detector responses with delayed neutron precursors shows an increased number of counts in interval III due to the contribution of delayed neutron emission. . . . . . . . . . . . . . .

3.1 A diagram of the slab geometry shows neutrons streaming through slab $n$ with direction $\mu \ldots \ldots . \ldots . \ldots . . . \ldots$

4.1 The TORTE free-gas scattering outgoing energy spectrum and prompt fission emission spectrum $\chi_{p}(E)$ agree with expected analytic distributions: the piecewise free-gas scattering kernel and Watt fission spectrum for thermal neutron-induced fission. . . . . . . . . . . . . . . 113

4.2 The $81 \alpha$ eigenvalues of Problem 3 form a circle in the complex plane. 118

4.3 Snapshots of the Problem 3 eigenfunction-expanded flux solution demonstrate the difference between the full solution using all 81 modes and the solution using only the fundamental mode. . . . . . . . . . .

4.4 The calculated 81-group variant $\alpha$-eigenvalue spectra with different group speeds (Problem 4) and downscattering over up to 5 groups (Problem 5) converge to analytic solutions at different rates. . . . . .

4.5 The $\alpha$ eigenvalue spectra of the 80 energy interval Problem 6 calculated using two random number seeds only differs for some higher complex eigenvalues. . . . . . . . . . . . . . . . . .

4.6 The $\alpha$-eigenvalue spectrum of the 600 energy interval Problem 6 has some similar spectral behavior as the 81-group problems, with some eigenvalues aligning along ellipses and curves. . . . . . . . . . . . . . 124 
4.7 The $\alpha$-eigenvalue spectrum of the 640 energy interval Problem 8 is completely negative and has a similar extent as the prompt supercritical problem but with fewer spectral features and more eigenvalues shifted towards the imaginary axis. . . . . . . . . . . . . . . . . .

4.8 The $\alpha$-eigenvalue spectra of Problem 6 calculated with different numbers of energy intervals agree for the first few eigenvalues but differ on the shape of the higher $\alpha$-eigenvalue spectrum. . . . . . . . . . . .

4.9 Two eigenvalues calculated with increasing numbers of energy intervals for Problem 6 shows the prompt eigenvalues asymptotically approaching a value, while the delayed eigenvalues remain the same. . . . . . .

4.10 The first calculated complex eigenvalue of Problem 6 approaches a value as the number of energy intervals increases. . . . . . . . . . .

4.11 Selected eigenfunctions of the 640 energy interval Problem 6 show the hard energy spectrum of the asymptotic solution, kinetic distortion of the delayed eigenfunction, and the importance of high-energy neutrons in a fast, prompt supercritical, infinite medium. . . . . . . . . .

4.12 An alternate visualization of the eigenfunctions of the 640 energy interval Problem 6 show the effect of the resonances on the forward and adjoint eigenfunctions. . . . . . . . . . . . . . .

4.13 The fundamental forward and adjoint eigenfunctions of the 3000 energy interval Problem 8 show the soft spectrum of the asymptotic solution and the importance of thermal neutrons in a thermal, graphitemoderated, subcritical infinite medium. . . . . . . . . . . . .

4.14 The next highest all-real, prompt forward eigenfunctions of the 3000 energy interval Problem 8 have similar shapes with positive and negative parts. . . . . . . . . . . . . . . . . .

4.15 Four of the first ten eigenvalues calculated after each active cycle converge as the number of active histories increases for the 320 energy interval Problem 7. . . . . . . . . . . . . . . .

4.17 Snapshots of the Problem 7 expanded flux solutions using all calculated modes show the agreement of the short-time evolution of the flux using different numbers of energy intervals during the TRM calculation. . .

4.18 Snapshots comparing the expanded flux solution to the TDMC solution for Problem 8 shows agreement throughout the transient for a graphitemoderated, subcritical problem reacting to a $14.1 \mathrm{MeV}$ pulse without initial precursor concentrations. . . . . . . . . . . .

4.19 Initial $t=0$ fluxes from the 3000 energy interval Problem 8 eigenfunction expansion show the magnitude of the missing residual term. . . .

4.20 The absolute value of the residual terms of the 3000 energy interval Problem 8 expanded flux solutions using two different initial sources have nearly identical shapes and the same magnitude relative to the initial source. . . . . . . . . . . . . . . . .

4.21 The absolute value of the initial residual terms for the three continuousenergy media have different shapes due to the material definitions. . . 
5.1 Snapshots compare the expanded flux solution to the TDMC solution, showing agreement throughout a transient in a scattering slab of thickness $\Delta=25.0 \mathrm{mfp}$.

5.2 The multi-region slab consists of several subregions of alternating ma-

5.3 Snapshots comparing the expanded flux solution to the TDMC solution agree throughout a transient in a multi-region slab with ten homogeneous 1-mfp subregions of alternating materials.

5.4 The TORTE-calculated fundamental shape eigenfunction of the tworegion problem matches that calculated at exact points by the GFM.

5.5 The five-region slab has loosely coupled fuel regions due to the large central absorber subregion. . . . . . . . . . . . . . .

5.6 The TORTE-calculated first two real shape eigenfunctions of the $\nu \Sigma_{f}=0.3$ five-region slab show the different scales of the fundamental and first shape eigenfunctions and the large effect of a small increase in the right fuel region thickness. . . . . . . . . . . . . . . .

5.7 The TORTE-calculated first two real shape eigenfunctions of the symmetric and asymmetric $\nu \Sigma_{f}=0.7$ five-region slabs match those calculated by the GFM. . . . . . . . . . . . . . . . . . . . . . . .

5.8 Snapshots of the expanded flux and TDMC solutions agree for a incident pulse on the subcritical, symmetric, $\nu \Sigma_{f}=0.7$ five-region slab. .

5.9 An alternate visualization of the expanded flux solution shows agreement with the TDMC solution for the ideal detector response at a point in the right fuel region. . . . . . . . . . . . . . . .

5.10 Snapshots of the expanded flux and TDMC solutions agree for an incident pulse on the supercritical, asymmetric, $\nu \Sigma_{f}=0.7$ five-region slab. . . . . . . . . . . . . . . . . . .

5.11 Snapshots of the expanded flux and TDMC solutions agree for a constant incident source on the very subcritical, symmetric, $\nu \Sigma_{f}=0.3$ five-region slab. . . . . . . . . . . . . . . . . . . . . . .

5.12 Snapshots of the expanded flux and TDMC solutions agree until oscillations appear at later times for a incident pulse on the very subcritical, symmetric, $\nu \Sigma_{f}=0.3$ five-region slab with a central void region. . . .

5.13 The size of the circular shape of the TORTE-calculated $\alpha$-eigenvalue spectrum of the $\Delta=5.0 \mathrm{mfp}$ thick scattering slab increases with the number of position intervals. . . . . . . . . . . . . .

5.14 There are $M / 2$ rings of eigenvalues in the TORTE-calculated $\alpha$ eigenvalue spectra of the $\Delta=5.0 \mathrm{mfp}$ thick scattering slab. . . . . . .

5.15 As the number of direction intervals increases to $M=64$, TORTE calculates additional rings of $\alpha$ eigenvalues that fill in a circle limited by the number of position intervals. . . . . . . . . . . . .

5.16 The first two TORTE-calculated eigenvalues of the $\Delta=5.0 \mathrm{mfp}$ thick scattering slab approach values as the number of position intervals increases. 
5.17 The first two TORTE-calculated eigenvalues of the $\Delta=5.0 \mathrm{mfp}$ thick scattering slab converge to values as the number of angular intervals increases.

5.18 The hydrogenous, seven-region slab consists of equal-sized homogeneous subregions of fuel and reflector materials. . . . . . . . . . . 181

5.19 The graphite-moderated, fifteen-region slab consists of homogeneous, equal-sized fuel subregions and larger outer and inner reflectors. . . .

5.20 The whole $\alpha$-eigenvalue spectrum of the graphite-moderated slab has different features that exist on very different scales. . . . . . . . . .

5.21 Snapshots comparing the spectra of the hydrogenous slab using different phase space discretizations show the effects of a change in the position, energy, or direction intervals on the TORTE-calculated $\alpha$ eigenvalue spectrum. . . . . . . . . . . . .

5.22 The fundamental forward and adjoint shape eigenfunctions of the hydrogenous slab all peak in the center of the fuel. . . . . . . . . . .

5.23 A comparison of the prompt fundamental and delayed fundamental shape eigenfunctions shows the small effect of kinetic distortion in the hydrogenous slab. . . . . . . . . . . . . . .

5.24 The first four thermal shape eigenfunctions $\phi_{n}$ of the graphitemoderated slab cross the $x$-axis $n$ times. . . . . . . . . . . .

5.25 Snapshots of the expanded flux solution show the propagation of the initial high-energy pulse and the increase in the thermal flux as these high-energy neutrons slow down in the hydrogenous slab. . . . . . . .

5.26 Responses of an ideal detector in the right-most fuel region shows a typical detector response obtained during a pulsed-neutron experiment (left) and one scaled to show the short-time effect of the high-energy pulse (right). . . . . . . . . . . . . . . . . . . . . . 191

6.1 Diffusion theory predicts the two-dimensional, prompt fundamental shape eigenfunctions of the hydrogenous cube. . . . . . . . . . .

6.2 The two-dimensional, prompt fundamental shape eigenfunction of the hydrogenous cube has a smooth, symmetric cosine shape. . . . . . . . 200

6.3 As OpenTRMM runs more particle histories for the hydrogenous cube, the first pair of multiple $\alpha$ eigenvalues converges as their corresponding shape eigenfunctions are still approaching the symmetric shapes predicted by diffusion theory. . . . . . . . . . . . . . . . .

6.4 Selected shape eigenfunctions of the hydrogenous cube demonstrate the progression of the first few prompt shape eigenfunctions. . . . . . 202

6.5 Snapshots of the relative detector response shape calculated from the expanded flux solution for the hydrogenous cube demonstrate the application of the TRMM in simulating time-dependent solutions. . . .

6.6 Responses of ideal detectors in two locations of the hydrogenous cube show the strong spatial dependence of the flux. . . . . . . . . . . 205 
6.7 A radial slice at the center of the approximate OpenMC model of the fast burst reactor CALIBAN shows the three control and excursion rods (light gray), four metal binding screws (dark gray), and the central irradiation channel. . . . . . . . . . . . . . . .

6.8 The trend of the largest delayed and prompt $\alpha$ eigenvalues with additional insertions of the BC3 control rod show the transition point at which the TRMM predicts the CALIBAN model switching from a subcritical to delayed supercritical state. . . . . . . . . . . . . .

6.9 The trend of the largest delayed and prompt $\alpha$ eigenvalue with additional insertion of the $\mathrm{BC} 2$ control rod show the transition point at which the TRMM predicts the CALIBAN model switching from a delayed supercritical to prompt supercritical state. . . . . . . . . .

6.10 An $r-\theta$ plot of the calculated fundamental prompt shape eigenfunction of the OpenMC CALIBAN model shows the Bessel function shape with no azimuthal variation. . . . . . . . . . . . . . . . . .

6.11 Plots in the $r-z$ space of the calculated fundamental and first prompt shape eigenfunctions shows the axial sine shape of the eigenfunctions corresponding to the $i_{z}=1$ an $i_{z}=2$ triplets. . . . . . . . . . .

6.12 Plots in the $r-\theta$ space of selected calculated prompt shape eigenfunctions of the OpenMC CALIBAN model show the different identifiable characteristics. . . . . . . . . . . . . . . .

6.13 Thirteen fuel compositions of the FSV initial core are arranged asymmetrically throughout the 37 fuel regions in an effort to better approximate the equilibrium core. . . . . . . . . . . . . .

6.14 The 37 control rod pairs at the center of each fuel regions fall into thirteen symmetric groups: twelve groups each have three control rod pairs, while the control rod pair in the first fuel region is in its own separate group. . . . . . . . . . . . . . . .

6.15 The OpenTRMM-calculated fundamental shape eigenfunction of the fully-rodded FSV reactor matches that calculated by General Atomics to help design the experiments. . . . . . . . . . . . . . .

6.16 Selected OpenTRMM-calculated thermal shape eigenfunctions of the FSV reactor show the progression of the oscillatory shapes as $n$ increases. 219

6.17 Selected OpenTRMM-calculated fast shape eigenfunctions of the FSV reactor show the progression of the oscillatory shapes as $n$ increases within the core. . . . . . . . . . . . . . . . .

6.18 The OpenTRMM-calculated detector responses show the strong spatial dependence between the three adjacent regions selected to analyze the pulsed neutron experiments. . . . . . . . . . . . . . . 


\section{LIST OF TABLES}

2.1 Six group delayed neutron data for ${ }^{235} \mathrm{U}$. . . . . . . . . . . . . 15

2.2 A summary of calculation procedures for space-time factorization methods. . . . . . . . . . . . . . . . . . 21

2.3 Comparisons of measured and MCNP5-calculated results for one subcritical configuration of the FSV PNE. . . . . . . . . . . . . . . 51

2.4 Summary of methods for calculating $\alpha$ eigenvalues, eigenfunctions, and kinetic modes.

3.1 True weighted constants of the discretized forward and adjoint $\alpha$ eigenvalue infinite medium problems. . . . . . . . . . . . . . . .

3.2 Approximated forward-weighted constants for the discretized adjoint $\alpha$-eigenvalue infinite medium problems. . . . . . . . . . . . . . . . . 100

3.3 Monte Carlo transition rate estimates for the general multidimensional problem. . . . . . . . . . . . . . 103

4.1 Problem 1 nuclear data. Cross sections are in $\mathrm{cm}^{-1}$. . . . . . . . . . 115

4.2 Problem 2 precursor data. . . . . . . . . . . . . . . . . 116

4.3 Problem 3 nuclear data. Cross sections are in $\mathrm{cm}^{-1}$. . . . . . . . . . 117

4.4 Nuclear data for the continuous-energy problems. . . . . . . . . . . . 122

4.5 A comparison of calculated delayed $\alpha$ eigenvalues. Units are $\mathrm{s}^{-1} \ldots . .125$

4.6 Problem 8 energy intervals and calculated prompt fundamental $\alpha$ eigenvalues. Units are $\mathrm{s}^{-1}$. . . . . . . . . . . . . . . . . . . . 128

4.7 Problem 7 energy intervals, calculated fundamental $\alpha$ eigenvalue, and first five prompt $\alpha$ eigenvalues. Units are $\mathrm{s}^{-1}$. . . . . . . . . . . . . . 129

4.8 Problem 7 calculated delayed $\alpha$ eigenvalues. Units are $\mathrm{s}^{-1}$. . . . . . . 129

4.9 A comparison of calculated neutron generation times of the three continuous-energy media. . . . . . . . . . . . . . . . . . . . 144

5.1 Simulated cross sections for obtaining the ideal detector response. . . 151

5.2 Comparison of TORTE- and GFM-calculated $\alpha$ eigenvalues for a homogeneous scattering slab. . . . . . . . . . . . . . . . . . . 153

5.3 TORTE- and GFM-calculated $\alpha$ eigenvalues for the multi-region slab. 156

5.4 Comparison of TORTE- and GFM-calculated $\alpha$ eigenvalues for small homogeneous multiplying slabs. . . . . . . . . . . . . . . . . . 159

5.5 Comparison of TORTE- and GFM-calculated $\alpha$ eigenvalues for large homogeneous multiplying slabs. . . . . . . . . . . . . . . . 160

5.6 Summary of the TORTE and GFM results for the five-region slab. . . 163 
5.7 The TORTE-calculated eigenvalues of the five-region slab with a central moderator replacing the absorber. . . . . . . . . . . . . 171

5.8 The largest TORTE-calculated $\alpha$ eigenvalues of the five-region slab with a central void replacing the absorber. . . . . . . . . . . . . . 172

5.9 Nuclear data for the continuous-energy slabs. . . . . . . . . . . . 180

5.10 Calculated neutron generation times of the two continuous-energy slabs. 182

5.11 Phase space discretization and calculated fundamental $\alpha$ eigenvalues of the hydrogenous continuous-energy slabs. . . . . . . . . . . . 184

5.12 Simulated cross sections for obtaining the ideal detector response of the hydrogenous slab. . . . . . . . . . . . . . . . . . . . . 191

6.1 Nuclear data for the hydrogenous cube. . . . . . . . . . . . . . 196

6.2 Position phase space discretization and calculated prompt fundamental $\alpha$ eigenvalues of the hydrogenous cube runs. . . . . . . . . . . . 197

6.3 The first few calculated prompt $\alpha$ eigenvalues of the hydrogenous cube. 198

6.4 Simulated cross sections for the hydrogenous cube. . . . . . . . . 203

6.5 Nuclear data for CALIBAN. . . . . . . . . . . . . . . . . . . 206

6.6 The first few calculated prompt $\alpha$ eigenvalues of the OpenMC CALIBAN model. . . . . . . . . . . . . . . . . . . . . . . 210

6.7 Total weight of uranium and thorium in the FSV fuel compositions. . 215

6.8 FSV pulsed-neutron experimental parameters. . . . . . . . . . . 217

6.9 FSV pulsed-neutron experiment results. . . . . . . . . . . . . 218 


\title{
LIST OF ABBREVIATIONS
}

\author{
ADS - accelerator-driven system \\ ANDA - active nondestructive assay \\ CCS - compressed column storage \\ CRS - compressed row storage \\ ENDF - evaluated nuclear data file \\ EPK - exact point kinetics \\ FOM - figure of merit \\ FP - fission product \\ FS - fixed source \\ FSV - Fort St. Vrain \\ GFM - Green's function method \\ HEU - high-enriched uranium \\ IQM - improved quasi-static method \\ IRAM - implicit-restarted Arnoldi method \\ LEU - low-enriched uranium \\ LWR - light water reactor \\ MC - Monte Carlo \\ MFP - mean free path \\ PBMR - Peach Bottom modular reactor \\ PCM - per cent mille \\ PCQM - predictor-corrector quasi-static method \\ PDF - probability density function \\ PK $\quad-$ point kinetics \\ PNE - pulsed neutron experiments \\ PPM - part per million \\ QM - quasi-static method \\ SNM - special nuclear material \\ SSI - sub-space iteration \\ TDMC - time-dependent Monte Carlo
}


TORTB - to obtain real time behavior

TORTE - to obtain real time eigenvalues

TRM - transition rate matrix

TRMM - transition rate matrix method 


\section{CHAPTER 1}

\section{Introduction}

Since the last half of the twentieth century, following Otto Hahn's discovery of fission in 1939 and the first experimental reactor built in 1942, nuclear science has grown to include various applications including agriculture, medicine, and engineering, producing ground-breaking technologies that greatly enhance quality of life [1]. Nuclear technologies, however, are subject to controversy and criticism ranging from irrational fears of radioactivity and cancer rates to legitimate concerns about the safety of nuclear systems and reactors, proliferation of nuclear weapons materials and methods, and handling nuclear reactor waste. The health of the industry relies on efforts to create safe practices and forward-thinking measures that benefit future generations for which these issues only become more important.

Fortunately, advancements in nuclear technology over the past fifty years provide engineers tools to eliminate or address many of these problems. Approximations

found in the earliest analysis tools for nuclear systems were a necessity because of the limited computational power in the mid-twentieth century. Increasingly sophisticated numerical methods developed over the years take advantage of improvements in nuclear and material data, computing power and memory, and the understanding of neutron behavior, producing general-purpose computer codes that are reliable and accurate [2].

This thesis describes a new numerical method in the field of neutron transport called the Transition Rate Matrix Method (TRMM). It uses solutions of the $\alpha$ - or time-eigenvalue problem obtained via a Monte Carlo matrix method for solving timedependent neutron transport problems. This introductory chapter discusses the general concepts of neutron transport, Monte Carlo methods, and describes engineering problems for which the TRMM is useful. 


\subsection{Neutron Transport}

Neutron transport is the motion of neutrons as they stream through material, frequently interacting with nuclei and eventually being absorbed or leaking out through a boundary. The fundamental equation describing this physical process is the linear Boltzmann transport equation, used in solving many nuclear engineering problems [3]. Assumptions made during its derivation are true for most applications and are omitted from this discussion. Many nuclear engineering problems fall into one of three categories: (i) deep penetration problems for designing shielding from a radioactive source; (ii) source-detector problems for designing devices to detect or measure output from a radioactive source; and (iii) criticality problems for nuclear reactor design.

In a transport problem, neutrons are born from sources and travel stochastically through the defined geometry. The life of a neutron consists of several events: (i) birth from a source or fission; (ii) collision events, in which the neutron interacts with a nucleus and either scatters to another direction and energy or is absorbed; (iii) leakage out of the material; and (iv) streaming between collisions. The transport equation has terms describing all of these physical phenomena. Numerically solving the transport equation with an appropriate initial condition and boundary conditions yields expected particle distributions within the geometry.

There are two classes of computational methods for solving the transport equation: deterministic and Monte Carlo. Deterministic methods discretize the position-energydirection-time phase space, building a system of equations that is solved iteratively. This discretization introduces truncation errors and is unable to treat irregular geometries or model small geometric details within a problem. Memory and time limitations sometimes require the representation of three-dimensional configurations as simplified two-dimensional models, introducing more sources of error. The generation of multigroup cross sections and appropriate homogenized cross sections complicates the process, and results in additional approximations. Monte Carlo methods treat the position-energy-direction-time phase space as continuous, providing for the treatment of complex, three-dimensional geometries and the elimination of truncation errors and multigroup approximations. Instead of attempting to solve the transport equation, Monte Carlo simulates the particle transport of a finite number of neutrons moving about the problem geometry. After generating enough stochastic particle trajectories, it extracts statistically significant quantities correct only within some uncertainty. Thus, Monte Carlo stochastic uncertainties replace the deterministic truncation errors [2]. Hybrid methods combine the best of both deterministic and Monte Carlo 
methods to improve performance and accuracy, and decrease calculation times.

For either class of method, solving the transport equation is computationally costly. A common simplification that reduces this cost is the steady-state assumption. It eliminates the temporal phase space, negating the time-dependent term and the time dependence of the neutron flux [3]. This assumption is valid for many problems already introduced: deep penetration problems, criticality calculations, and many source-detector problems. For some fixed-source problems, the desired solutions are essentially probabilities independent of time. In reactor design, the steady-state assumption is only natural: nuclear power reactors operate at steady-state, with a sustained nuclear chain reaction. Under normal operating conditions, changes in reactors occur over weeks and months, timescales for which solutions to the time-dependent transport equation are unnecessary.

But, there are many nuclear applications where relatively short time-dependent phenomena are of particular interest. For these problems, the steady-state assumption is invalid and the time-dependent form of the transport equation yields the appropriate solution. This time-dependent behavior is important for analysis of pulsedneutron experiments and nuclear reactor kinetics. Kinetics is the study of nuclear reactor transients, and is important for analysis of accident scenarios. Obtaining solutions of the time-dependent transport equation are even more computationally demanding. The characteristic times at which nuclear systems evolve are on the order of a mean neutron life time, $10^{-8}$ to $10^{-4}$ seconds depending on the problem materials. With time steps relative to this scale, even lengthy computations only calculate a solution representing a fraction of a second. Thus, approximations are necessary to asses many of these problems [2]. The next chapter discusses some of these approximate methods in depth. For now, it is worth introducing the Monte Carlo method.

\subsection{The Monte Carlo Method}

Modern applications of the Monte Carlo method for particle transport began in the 1940's, facilitated by the development of the first digital computers [4]. The method applies to both photons and neutrons, but here the discussion focuses on neutron transport. It is unique for the use of random sampling to construct the solution to the transport equation, as opposed to discretizing it to approximate a numerical solution. Though computationally intensive, with ongoing advancements in processors, memory, and parallel architecture, the Monte Carlo method is one of the more ac- 
curate tools in reactor physics; often Monte Carlo solutions serve as a reference for validation of new deterministic tools.

The Monte Carlo method uses computer algorithms that generate a sequence of pseudo-random numbers. These pseudo-random number generators compute a unique sequence for a given random number seed: to repeat a sequence, the algorithm uses the same seed. Pseudo-random numbers are sampled uniformly $\in(0,1)$ and are denoted mathematically as $\xi$. Though these numbers are only pseudo-random, algorithms available today generate a sequence that is sufficiently random for most applications [5]. The Monte Carlo method uses these random numbers in simulating the sequence of events in the life of a neutron.

Neutron transport is a stochastic process in which a neutron undergoes a series of random events from birth to destruction or leakage from a problem. Probabilities determine this sequence of events, known as the neutron history. During the simulation of neutron histories, Monte Carlo tallies extract information, generating averaged results close to true solutions within some statistical uncertainty.

To implement the TRMM, this thesis uses the Monte Carlo method because of its ability to describe the behavior of neutrons moving through any problem. Though the TRMM discretizes the phase space when performing matrix calculations, it otherwise maintains a continuous space and stochastic approach that is characteristic of a Monte Carlo random walk. The discretization applies only to tallies. This treatment allows the TRMM to change the discretization of the phase space to fit the intended purpose.

For the first two parts of the thesis, a simplified, research Monte Carlo code implements the TRMM for infinite and one-dimensional media for observing trends, convergence behavior, and accuracy. The final part of this thesis incorporates the TRMM into a documented Monte Carlo code to study the applicability, viability, and accuracy of the method using full three-dimensional models and measured data.

\subsection{Applications for the Transition Rate Matrix Method}

The TRMM applies to problems where the time-dependence of the neutron flux is of interest. Many of these are source-detector problems, where the desired solution is the time-dependent detector or flux response to some arbitrary source in the system. These source-detector problems are subcritical, meaning that the neutron flux decreases with passing time. Conversely, the neutron flux increases with time in supercritical problems. The TRMM also applies to these problems because it calculates $\alpha$ eigenvalues [6], but this thesis is limited to subcritical and near-critical systems where 
data and benchmark calculations are available. This section focuses on a few of the potential applications of this research: active nondestructive assay, pulsed-neutron experiments, accelerator-driven subcritical systems, and fast-burst reactors.

\subsubsection{Active Nondestructive Assay}

Nuclear materials emit characteristic radiation both spontaneously and when irradiated by neutrons or $\gamma$-rays. Passive or active nondestructive assay is the measurement of this spontaneous or induced radiation to determine the amount or type of material in a sample without affecting its physical form [7]. Recent research on active nondestructive assay (ANDA) focuses on its applicability to nuclear material accounting, that is, safeguards against the diversion of special nuclear material (SNM), like high enriched ${ }^{235} \mathrm{U}$ and ${ }^{233} \mathrm{U}$, and plutonium. In such security applications, a conservative scenario assumes that SNM is sufficiently shielded to prevent passive detection [8].

In a safeguards application, a source with a selected strength and energy irradiates an unknown material and configuration. Detectors placed about the configuration record the response to the source: the analysis of this response reveals the presence or absence of SNM by detecting induced fissions. Analysis techniques fall into two categories: detection of delayed or prompt radiation [7]. Induced fissions in SNM emit prompt neutrons and $\gamma$-rays immediately after irradiation. It is important to differentiate this prompt radiation from the initial source. Delayed neutrons and $\gamma$ rays come from short-lived fission products: these nuclei decay at a much later time than prompt radiation emission, but are produced in much smaller quantities.

For either technique, ANDA is a time-dependent source-detector problem to which the TRMM is applicable. The TRMM accounts for delayed fission and distinguishes between delayed neutron release and prompt neutron effects. It is also able to capture very short-time phenomena useful in differentiating between prompt radiation from fission and radiation from the initial source.

\subsubsection{Pulsed-Neutron Experiments}

Pulsed-neutron [9] and Rossi- $\alpha$ [10] experiments are similar problems that measure the decay time of neutrons within a system. These experiments provide a way of measuring the reactivity of subcritical systems, studying the time-dependent behavior of the flux, verifying calculated physics behavior in commercial reactors [11], and measuring certain kinetics parameters [12]. Pulsed-source experiments have many other applications: evaluating cross sections $[13,14]$, studying neutron slowing down 
in moderators [15, 16], measuring control rod worth [17], measuring diffusion parameters $[18,19]$, and obtaining the prompt decay mode of reactors or other assemblies [20]. Even ANDA problems are pulsed-source systems. This section focuses on pulsed-source experiments on reactor configurations.

During or after the initial fuel loading of a reactor, pulsed-neutron experiments (PNE) measure the subcriticality of a partially- or fully-loaded core with a given control rod configuration. The configurations chosen reflect possible scenarios during normal operation or those with safety implications [21]. These measurements assess that the fuel is properly loaded and that computer models have accurately predicted, among many things, the core reactivity, control rod worths, and shutdown margins. This allows for proper adjustments to the nuclear design or operational procedures in case of a severely inaccurate model.

Several power reactors used PNE for these purposes [22, 23]. General Atomics performed PNE on the Peach Bottom Modular Reactor (PBMR) [24] and the Fort St. Vrain (FSV) [25] reactor after the initial fuel was loaded into the core. These are both graphite-moderated, gas-cooled reactor designs: the PBMR was a proof-ofconcept for the larger FSV reactor, which had a mixed operational history after first delivering commercial power in Colorado in the late 1970's [26].

In a PNE, a source instantaneously inserts a large number of neutrons into the reactor and detectors placed in different regions measure the flux time response (Figure 1.1). Analysis of the detector response curve reveals the level of criticality of the core [11]. Depending on the size of the reactor, the spatial dependence of the flux may vary greatly, even though it is reacting to the same pulsed source. For example, detectors in different regions of FSV experience greatly different responses (Figure 1.1) due to a pulsed source. Both the sheer size of FSV as well as the heterogeneity of the core contribute to these differences. In smaller systems, such as the PBMR, the flux varies less spatially [27]. Note also that the responses of each of the detectors exhibit a similar, exponential time-dependent trend.

Regardless of the application, pulsed-source experiments are time-dependent source-detector problems to which the TRMM is applicable. For PNE, the TRMM calculates the space-time dependence of the neutron flux. Several methods that calculate the reactivity from the detector response attempt to account for these spatial differences [28]. The TRMM calculates the spatial information necessary to predict these differences. The next chapter has an in-depth discussion on the technical aspects of PNE. 


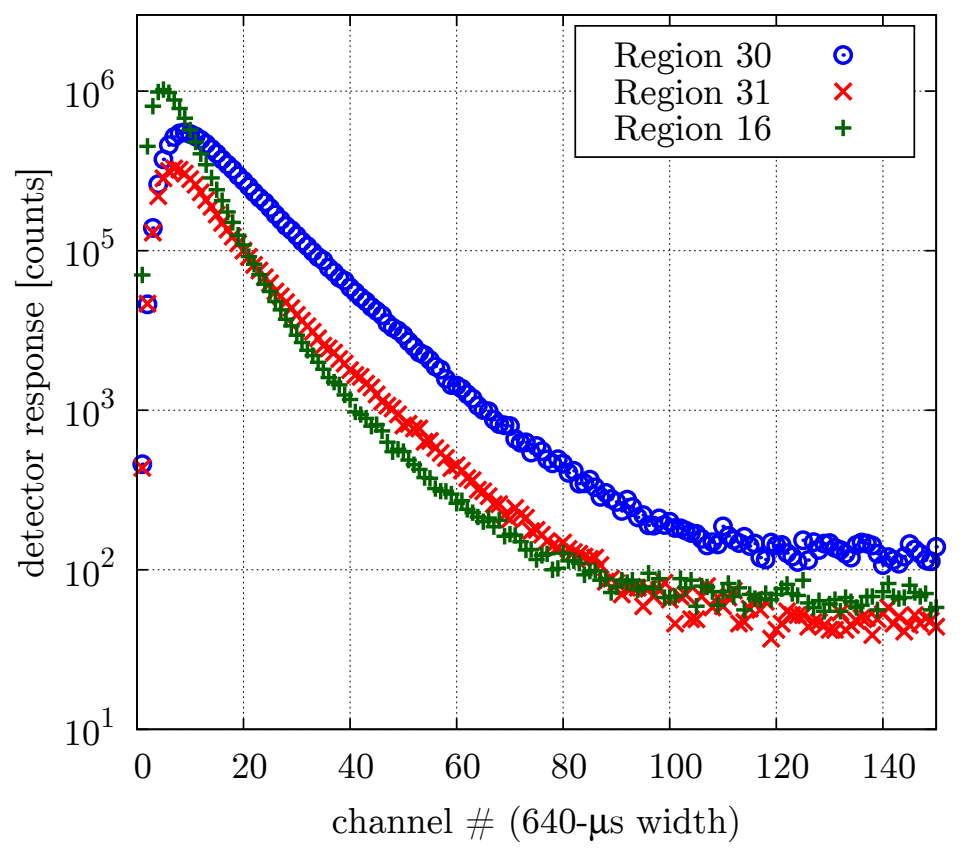

Figure 1.1: Measured time-dependent responses of three different detectors for a subcritical control rod configuration of Fort St. Vrain demonstrate the strong spatial dependence of the neutron flux for large systems.

\subsubsection{Accelerator-Driven Subcritical Systems}

Accelerator-driven systems (ADS) are subcritical configurations of multiplying material pulsed with large numbers of neutrons generated by an accelerator imparting protons or ions onto a spallation target. There is renewed interest in ADS due to nuclear reactor waste disposal concerns: these systems transmute radioactive nuclei to other isotopes that are stable or have shorter half lives, providing an alternative to geologic, ice sheet, or outer space disposal. An ADS generates enough high-energy neutrons for the transmutation of most long-lived actinides and fission products, as well as enough power to supply the accelerator [29]. Also, supercritical excursions, like that at the Chernobyl reactor, are impossible in ADS.

The ADS fits into a more complete approach to the treatment of nuclear waste. Currently, the direct core-to-storage fuel cycle has commercial light water reactors (LWR) producing waste requiring storage for at least several hundred years. A more complete fuel cycle reduces this storage time by transmuting actinides in spent fuel with fast reactors or ADS, with the latter having some neutronic advantages [30].

Current LWR and most other power reactors operate at a critical condition; many reactor physics tools serve the purpose of analyzing these steady-state systems. These tools are insufficient for treating ADS: in subcritical configurations, the neutron flux 
will always vary with time except in the presence of a constant external source. Characterizing this space-time dependence of the flux is important to design calculations and requires solutions to the time-dependent transport equation. Recent ADS experimental programs in Europe demonstrate the interest in ADS and their potential for use in future nuclear waste cycles: the MUltiplication avec Source Externe (MUSE) experimental program in France [31] and the YALINA experimental program in the former Soviet Union [32].

The ADS is a source-driven problem similar to the PNE described in the previous section to which the TRMM is well-suited. The difference is that the overall spatial flux response in an ADS is of interest. Still, detector responses from a pulse on an ADS reveals spatial dependences and are important for monitoring the reactivity of the configuration [33]. The TRMM obtains these spatial dependences and calculates the overall space-time response of the neutron flux for an ADS with the appropriate source. Also, analysis of transients in an ADS is important in assessing reactor safety: the TRMM provides information desirable in quantifying rapid changes in the system [34].

\subsubsection{Fast-Burst Reactors}

Fast-burst reactors generate short bursts of radiation used for testing and experimentation. Many of these systems are made up of several pieces of HEU alloy that are placed together to cause a rapid increase in the neutron flux, generating very high-flux neutron pulses. The system returns to a subcritical state due to thermal and geometric feedback. For specific experiments, some fast-burst reactors are able to operate in continuous mode: at subcritical steady-state or at delayed critical [35]. Some reactors have holes for sample irradiation. Examples of these assemblies include CALIBAN [36], Molly-G, SFR-I and SFR-II.

Worldwide, an estimated 61 tons of HEU is under civilian use, most in reactor applications. This presents a safeguards concern: much of the HEU is in facilities with inadequate security and only $25 \mathrm{~kg}$ of ${ }^{235} \mathrm{U}$ is necessary to produce a nuclear weapon. For these reasons, national agencies encourage the conversion of HEU reactors to lowenriched uranium (LEU) [37]. In the United States, regulation of HEU is stringent: armed personnel are present during experiments and carefully monitor the storage and handling of the material. Thus, in the US, this conversion has an additional costreduction benefit and is less cumbersome to researchers working with the material, assuming that LEU is a sufficient substitute for the particular application.

For any system, a HEU-LEU conversion requires many design changes to accom- 
modate the lower enrichment. The immediate effect for fast-burst reactors is an increase in the size of the system. The amount of fissile ${ }^{235} \mathrm{U}$ must be preserved, so more LEU is necessary to obtain criticality. In these bare systems, the uranium is already in a dense metallic form, so one of the few design changes possible is to make the LEU assembly physically larger. For the larger system, the spatial time-dependence of the neutron flux within the reactor becomes more important, much like in the PBMR-FSV comparison. The TRMM calculates this time dependence and the time it takes for the neutron flux within the reactor to achieve its asymptotic behavior. Also, fast burst reactors are geometrically simple problems: analytical back-of-theenvelope calculations provide some solutions to give validity to the TRMM.

\subsection{Thesis Objective and Outline}

The objective of this thesis is to demonstrate the Transition Rate Matrix Method for calculating $\alpha$ eigenvalues and eigenfunctions of a nuclear system using Monte Carlo, while identifying its strengths and weaknesses. Preceding material in this introduction provides discussions on neutron transport, the Monte Carlo method, and potential applications for this thesis work. The remainder of the thesis proceeds as follows:

- Chapter 2 discusses topics important to time-dependent neutron transport theory: current methods for time-dependent reactor analysis, such as point kinetics, quasi-static treatments, modal expansion, and time-dependent Monte Carlo; the $k$-, $c$-, and $\alpha$-eigenvalue problems, with derivations and discussions of characteristics of the $\alpha$-eigenvalue spectrum and eigenfunction expansion; and known methods for measuring and calculating $\alpha$ eigenvalues and eigenfunctions.

- Chapter 3 explains the new transition rate matrix tally approach developed to calculate $\alpha$ eigenvalues and eigenfunctions with Monte Carlo, starting from theory: introduction to Markov processes; the time-dependent neutron importance equation; the theoretical basis of the transition rate matrix; similarities to the fission matrix method; derivations of the transition rate matrix for infinite and one-dimensional media; estimation of the matrix elements with Monte Carlo tallies; and a brief discussion on eigenvalue solvers.

- Chapter 4 presents TRMM results from calculating the $\alpha$-eigenvalue spectrum for infinite, i.e., 0-D, media with a simplified research Monte Carlo transport code, covering several topics: details of the Monte Carlo code physics; verifications for multigroup problems; continuous-energy problems of hydrogenous and graphite multiplying media with comparisons to time-dependent Monte Carlo 
solutions; discussion on the effect of discretization of the phase space; and convergence observations of the $\alpha$-eigenvalue spectrum.

- Chapter 5 shows the implementation of the TRMM for one-dimensional media, including several topics: details of the Monte Carlo code physics; verifications to the Green's Function Method of calculating $\alpha$ eigenvalues and eigenfunctions; comparisons to time-dependent Monte Carlo solutions; comments on the behavior of the $\alpha$-eigenvalue spectrum; discussions on choice of discretization of the phase space; and convergence observations.

- Chapter 6 demonstrates the application of the TRMM to a physical problem, with additional discussions: the implementation of the TRMM into OpenMC; comparisons to $\alpha$ eigenvalues and eigenfunctions obtained with diffusion theory; verifications to measured data for pulsed reactor systems and fast-burst reactors; and discussions on runtime and convergence of the $\alpha$ eigenvalues and eigenfunctions.

- Chapter 7 summarizes the important contributions achieved in this thesis, and makes closing comments on performance, usability, and future directions for this method.

\section{References}

[1] J. K. Shultis and R. E. Faw, Fundamentals of Nuclear Science and Engineering. New York: Marcel Dekker, 2002.

[2] E. E. Lewis and W. F. Miller, Computational Methods of Neutron Transport. New York: John Wiley \& Sons, 1984.

[3] J. J. Duderstadt and L. J. Hamilton, Nuclear Reactor Analysis. New York: John Wiley \& Sons, 1976.

[4] N. Metropolis, "The Beginning of the Monte Carlo Method," Los Alamos Science, vol. Special Issue, pp. 125-130, 1987.

[5] Y. Nagaya and F. B. Brown, "Testing MCNP Random Number Generators," Tech. Rep. LA-UR-11-04858, Los Alamos National Laboratory, 2011.

[6] D. Brockway, P. Soran, and P. Whalen, "Monte-Carlo Eigenvalue Calculation." Los Alamos National Laboratory, 1985.

[7] T. Gozani, Active Nondestructive Assay of Nuclear Materials. Washington, D. C.: U. S. Nuclear Regulatory Commission, 1981.

[8] H. Yang, Active Interrogation Methods for Detection of Special Nuclear Material. PhD thesis, University of Michigan, 2009.

[9] N. G. Sjöstrand, "Measurements on a Subcritical Reactor Using a Pulse Neutron Source," Arkiv Fysik, vol. 11, p. 223, 1956.

[10] G. E. Hansen, "The Rossi Alpha Method," Tech. Rep. LA-UR-85-4176, Los Alamos National Laboratory, 1985. 
[11] W. Pfeiffer, J. R. Brown, and A. C. Marshall, "Fort St. Vrain Startup Test A-3: Pulsed-Neutron Experiments," Tech. Rep. GA-A13079, General Atomic, 1974.

[12] G. D. Spriggs, "Two Rossi- $\alpha$ Techniques for Measuring the Effective Delayed Neutron Fraction," Nuclear Science and Engineering, vol. 113, pp. 161-172, 1993.

[13] J. W. Meadows and J. F. Whalen, "Thermal Neutron Absorption Cross Sections by the Pulsed Source Method," Nuclear Science and Engineering, vol. 9, pp. 132$136,1961$.

[14] I. E. Beghian, N. V. Rasmussen, R. Thews, and J. Weber, "The investigation of Neutron Kinetics and Cross Sections in Fast Non Moderating Assemblies by the Nanosecond Pulsed Neutron Source Technique," Nuclear Science and Engineering, vol. 15, pp. 375-381, 1963.

[15] E. Friedman, "Studies of Neutron Thermalization in $\mathrm{H}_{2} \mathrm{O}$ by the Pulsed Source and "Non $\frac{1}{v "}$ Absorber Method," Nuclear Science and Engineering, vol. 19, pp. 203-208, 1964.

[16] A. E. Profio and J. D. Eckard, "Investigation of Neutron Moderation with a Pulsed Source," Nuclear Science and Engineering, vol. 19, pp. 321-328, 1964.

[17] O. C. Kolar and F. A. Kloverstrom, "Pulsed Neutron Measurement of Control Rod Worths," Nuclear Science and Engineering, vol. 10, pp. 25-52, 1961.

[18] W. M. Lopez and J. R. Beyester, "Measurement of Neutron Diffusion Parameters in Water by the Pulsed Neutron Method," Nuclear Science and Engineering, vol. 12, pp. 190-202, 1961.

[19] W. W. Clendenin, "Relation of the Neutron Diffusion Length to Neutron-Pulse Parameters in $\mathrm{H}_{2} \mathrm{O}$," Nuclear Science and Engineering, vol. 20, pp. 248-249, 1964.

[20] C. Sastre and E. V. Weinstock, "A Note on Delayed-Neutron Effects in PulsedNeutron Measurements on Multiplying Assemblies," Nuclear Science and Engineering, vol. 20, pp. 359-364, 1964.

[21] J. R. Brown, "Pulsed Source Measurements of Control Rod Worth in Peach Bottom," Tech. Rep. GAMD-7352, General Atomic, 1966.

[22] S. Fuster, A. Tarabella, and H. Tellier, "Experiences de Pulsations Neutroniques sur le Reacteur a Graphite EDF 3," Tech. Rep. CEA-R 3162, Commissariat à l'Energie Atomique, Saclay, France, 1967.

[23] I. Brittain, "Analysis of Rod Drop and Pulsed Source Measurements of Reactivity in the Winfrith SGHWR," Tech. Rep. AEEW-R 640, Atomic Energy Establishment, Winfrith, England, 1970.

[24] C. A. Preskitt, E. A. Nephew, J. R. Brown, and K. R. V. Howe, "Interpretation of Pulsed-Source Experiments in the Peach Bottom HTGR," Nuclear Science and Engineering, vol. 29, pp. 283-295, 1967.

[25] S. R. Ross, J. R. Brown, and R. J. Nirschl, "Startup Physics Tests at Fort St. Vrain," Tech. Rep. GA-A13487, General Atomic, 1975.

[26] B. R. Betzler, J. C. Lee, and W. R. Martin, "MCNP5 Analysis of Fort St. Vrain High-Temperature Gas-Cooled Reactor," in Trans. Am. Nucl. Soc., vol. 102, p. 515, 2010.

[27] W. Pfeiffer, J. R. Brown, and A. C. Marshall, "Analysis and Results of PulsedNeutron Experiments on the Fort St. Vrain HTGR," Tech. Rep. GA-A13342, 
General Atomic, 1975.

[28] Y. Cao and J. C. Lee, "Spatial Corrections for Pulsed-Neutron Reactivity Measurements," Nuclear Science and Engineering, vol. 165, pp. 270-282, 2010.

[29] R. G. Cochran and N. Tsoulfanidis, The Nuclear Fuel Cycle: Analysis and Management. La Grange Park, Illinois: American Nuclear Society, 1999.

[30] M. Salvatores, I. Slessarev, and M. Uematsu, "A Global Physics Approach to Transmutation of Radioactive Nuclei," Nuclear Science and Engineering, vol. 116, pp. 1-18, 1994.

[31] Nuclear Energy Agency, "Benchmark on Computer Simulation of MASURCA Critical and Subcritical Experiments: MUSE-4 Benchmark, Final Report," in NEA/NSC/DOC(2005)23, OECD, 2006.

[32] Y. Gohar and D. L. Smith, "YALINA Facility A Sub-Critical Accelerator-Driven System (ADS) for Nuclear-Energy Research Facility Description and an Overview of the Research Program (1997-2008)," Tech. Rep. ANL-10/05, Argonne National Laboratory, 2010.

[33] C. M. Persson, "Pulsed Neutron Source Measurements in the Subcritical ADS Experiment YALINA-Booster," Annals of Nuclear Energy, vol. 35, p. 2357, 2008.

[34] F. Wols, "Transient Analyses of Accelerator Driven Systems Using Modal Expansion Techniques," Master's thesis, Delft University of Technology, PNR-1312010-005, 2010.

[35] P. Humbert and B. Méchitoua, "Numerical Simulation of CALIBAN Reactivity Perturbation Experiments Using Neptunium-237 Samples," in Proc. Nuclear Mathematical and Computational Sciences: A Century in Review, A Century Anew, (Gatlinburg, Tennessee), April 2003.

[36] N. Authier and B. Méchitoua, "Bare, Highly Enriched Uranium Fast Burst Reactor CALIBAN," in NEA/NSC/DOC/(95)03/II, vol. II, HEU-MET-FAST-080, ICSBEP, 2007.

[37] James Martin Center for Nonproliferation Studies, "Civilian HEU Reduction and Elimination Resource Collection," Nuclear Treat Initiative, 2013. 


\section{CHAPTER 2}

\section{Time-Dependent Neutron Transport}

Methods for solving the steady-state transport equation predict critical configurations, spatial flux distributions, and reaction rates for time-independent problems [1]. The steady-state treatment serves well for these problems, however, this assumption is invalid for non-critical systems where the neutron production and losses are unbalanced. For example, in a nuclear reactor where the combined neutron absorption and leakage rate is greater than the fission source rate, the neutron population decreases with passing time. These unbalanced states occur during normal operations: startup, shutdown, fuel loading, and changing power levels. They also occur during unexpected transients: rod ejections and coolant pump failures [2]. In general, the study of these time-dependent problems is known as kinetics. Reactor dynamics is distinguishable from kinetics because it includes feedback mechanisms omitted from this discussion.

Because direct solutions to the time-dependent transport equation are prohibitively costly, many kinetics methods factorize the flux solution into a timedependent amplitude function and a position-energy-direction-time-dependent shape function. The amplitude function describes the majority of the time variation of the flux, while the shape function describes the spatial, energy, and angular dependences and varies very little in time. Using this separation simplifies computation because the amplitude function is much easier to obtain than the shape function. With additional approximations, these methods are applicable for describing a range of reactor kinetics problems, particularly those where the flux variation in time consists primarily of flux amplitude changes [3]. For some applications, these methods are unable to appropriately describe the space-time evolution of the flux. In contrast to these methods is time-dependent Monte Carlo, which is incredibly costly computationally, but is able to accurately describe the flux-time behavior with a sufficient number of neutron histories. 
The $\alpha$, or time, eigenvalue is an important parameter for describing timedependent neutron transport. Knowledge of the entire $\alpha$-eigenvalue spectrum, that is, all the $\alpha$ 's that balance the transport equation, provides the time-dependent flux response to any arbitrary source via eigenfunction expansion [4]. Higher $\alpha$ eigenfunctions describe the spatial differences in the flux response for a configuration subject to an external source.

The TRMM uses the concepts introduced above to solve time-dependent neutron transport problems. It calculates $\alpha$ eigenvalues and eigenfunctions using Monte Carlo, and uses eigenfunction expansion to solve for the time-dependent flux in kinetics problems. Therefore, it is useful to discuss some of these concepts in depth: the timedependent transport equation, current methods for solving kinetics problems, the $\alpha$ eigenvalue problem, and current methods for calculating and measuring $\alpha$ eigenvalues.

\subsection{Time-Dependent Equations}

The time-dependent neutron transport and precursor equations [1] are

$$
\begin{aligned}
\frac{1}{v} \frac{\partial \psi}{\partial t}+\hat{\mathbf{\Omega}} \cdot \nabla \psi+ & \Sigma \psi(\mathbf{r}, E, \hat{\mathbf{\Omega}}, t) \\
= & \iint \Sigma_{s}\left(E^{\prime}, \hat{\mathbf{\Omega}}^{\prime} \rightarrow E, \hat{\mathbf{\Omega}}\right) \psi^{\prime} d E^{\prime} d \mathbf{\Omega}^{\prime} \\
& +\iint \frac{\chi_{p}}{4 \pi}(1-\beta) \bar{\nu} \Sigma_{f} \psi^{\prime} d E^{\prime} d \mathbf{\Omega}^{\prime}+\sum_{j}^{J} \frac{\chi_{j}}{4 \pi} \lambda_{j} C_{j}+\frac{S}{4 \pi}, \\
\frac{\partial C_{j}}{\partial t}+\lambda_{j} C_{j}(\mathbf{r}, t)= & \iint \beta_{j} \bar{\nu} \Sigma_{f} \psi^{\prime} d E^{\prime} d \mathbf{\Omega}^{\prime}, \quad \text { for } \quad j=1, \ldots, J,
\end{aligned}
$$

where $\psi$ is the angular flux, $C_{j}$ is the precursor concentration, $j$ denotes the precursor group, and $\mathbf{r}, E, \hat{\Omega}, t$ describes the position-energy-direction-time phase space. The $v$ is the neutron speed, $\Sigma$ is the total macroscopic cross section, $\Sigma_{s}$ is the scattering cross section from $E^{\prime}, \hat{\Omega}^{\prime}$ to $E, \hat{\Omega}, \chi_{p}$ is the prompt fission emission spectrum, $\beta$ is the total delayed neutron fraction, $\bar{\nu}$ is the average neutrons released per fission, $\Sigma_{f}$ is the fission cross section, $\chi_{j}$ is the delayed neutron emission spectrum, $\lambda_{j}$ is the delayed precursor decay constant, $S$ is the isotropic external source, and $\beta_{j}$ is the group delayed neutron fraction.

The first term of Eq. (2.1) is the time-dependent variation of the flux, normally omitted in the steady-state formulation. The next six terms describe neutron interactions with material: leakage from the phase space, collisions, in-scatter, prompt fission, delayed fission, and external source. For simplicity, this formulation uses 
precursor density equations to describe delayed neutron emission. Accounting for delayed neutron emission within Eq. (2.1) requires delta functions and an integral over all past times, making it less convenient. For each group j, Eq. (2.2) describes the time-dependent variation of the precursor density with two terms: precursor loss due to decay, and precursor source from fission.

Delayed neutron emission occurs as a result of typically less than $1 \%$ of fissions. When a heavy nucleus fissions, it splits into two or more fission products (FP) and neutrons. A small percentage of these fissions release a FP known as a delayed neutron precursor. Delayed neutron precursors are radioactive, and upon decaying release a neutron known as a delayed neutron. The release time of a delayed neutron depends on the half-life of the delayed neutron precursor. The energy of a delayed neutron is often much less than a prompt fission neutron and depends on the excited state of the delayed neutron precursor [3]. For ${ }^{235} \mathrm{U}$, nuclear data sheets categorize the approximately 40 delayed neutron precursors into $J=6$ groups according to their half-lives [5]. Each group has its own characteristic energy distribution, decay constant, and emission probability (Table 2.1). Most formulations in this thesis use the unnormalized $\beta_{j}$.

Delayed neutron emission strongly influences the time-dependence of the flux even though they only account for a small percentage of neutrons born from fission. This is because their emission time, ranging from a few seconds to several minutes after the initial fission, is much longer than the prompt neutron lifetime of $10^{-8}$ to $10^{-4}$ seconds [6]. This wide disparity in characteristic times complicates computation, but is very important for reactor control. With only prompt neutrons, a slight change in the problem has a nearly immediate effect on the flux. Delayed neutrons provide a time bottleneck, causing a lagged response in the flux on the order of a few seconds, enough time to incorporate desired control mechanisms [7]. For example, the prompt neutron chain reaction ceases after reactor shutdown, but, there is a time-dependent neutron source that remains in the reactor: the decay of the neutron precursors

Table 2.1: Six group delayed neutron data for ${ }^{235} \mathrm{U}$.

\begin{tabular}{ccc}
\hline$j$ & $\lambda_{j}\left[\mathrm{~s}^{-1}\right]$ & $\beta_{j}$ [normalized] \\
\hline 1 & 0.0125 & 0.0320 \\
2 & 0.0318 & 0.1664 \\
3 & 0.109 & 0.1613 \\
4 & 0.317 & 0.4596 \\
5 & 1.35 & 0.1335 \\
6 & 8.64 & 0.0472 \\
\hline
\end{tabular}


accumulated during reactor operation. Therefore, a reactor shuts down only as fast as the decay of the longest-lived precursors.

\subsection{Solutions to the Neutron Balance Equation}

To simplify the notation in Eqs. (2.1) and (2.2), introduce the operator notations

$$
\begin{aligned}
\mathbf{L} \psi(\mathbf{r}, E, \hat{\mathbf{\Omega}}, t) & =\hat{\mathbf{\Omega}} \cdot \nabla \psi, \\
\mathbf{R} \psi & =\Sigma(\mathbf{r}, E) \psi, \\
\mathbf{S} \psi & =\iint \Sigma_{s}\left(\mathbf{r} ; E^{\prime}, \hat{\mathbf{\Omega}}^{\prime} \rightarrow E, \hat{\mathbf{\Omega}}\right) \psi\left(\mathbf{r}, E^{\prime}, \hat{\mathbf{\Omega}}^{\prime}, t\right) d E^{\prime} d \mathbf{\Omega}^{\prime}, \\
\mathbf{M} \psi & =\mathbf{L} \psi+\mathbf{R} \psi-\mathbf{S} \psi, \\
\mathbf{F}_{\mathbf{p}} \psi & =\iint \frac{\chi_{p}(E)}{4 \pi}(1-\beta) \bar{\nu}\left(E^{\prime}\right) \Sigma_{f}\left(\mathbf{r}, E^{\prime}\right) \psi\left(\mathbf{r}, E^{\prime}, \hat{\mathbf{\Omega}}^{\prime}, t\right) d E^{\prime} d \mathbf{\Omega}^{\prime}, \\
\mathbf{F}_{\mathbf{d} j} \psi & =\iint \beta_{j} \bar{\nu}\left(E^{\prime}\right) \Sigma_{f}\left(\mathbf{r}, E^{\prime}\right) \psi\left(\mathbf{r}, E^{\prime}, \hat{\mathbf{\Omega}}^{\prime}, t\right) d E^{\prime} d \mathbf{\Omega}^{\prime} .
\end{aligned}
$$

where the $\mathbf{M}$ operator combines the leakage $\mathbf{L}$, collision $\mathbf{R}$, and scattering $\mathbf{S}$ terms, and the prompt and group $j$ delayed fission operators are $\mathbf{F}_{\mathbf{p}}$ and $\mathbf{F}_{\mathbf{d} j}$, respectively. For many reactor applications, the angular details of the flux are unnecessary, and the scalar flux

$$
\phi(\mathbf{r}, E, t)=\int_{4 \pi} \psi(\mathbf{r}, E, \hat{\mathbf{\Omega}}, t) d \mathbf{\Omega},
$$

is sufficient for calculating reaction rates and determining critical configurations. Integrating Eqs. (2.1) and (2.2) over all angles $\int_{4 \pi}(\cdot) d \boldsymbol{\Omega}$ and introducing operator notation yields the time-dependent neutron continuity or diffusion equations [2] with precursors

$$
\begin{aligned}
\frac{1}{v} \frac{\partial \phi}{\partial t}+\mathbf{M} \phi(\mathbf{r}, E, t) & =\mathbf{F}_{\mathbf{p}} \phi+\sum_{j}^{J} \chi_{j} \lambda_{j} C_{j}+S, \\
\frac{\partial C_{j}}{\partial t}+\lambda_{j} C_{j}(\mathbf{r}, t) & =\mathbf{F}_{\mathbf{d} j} \phi, \quad \text { for } \quad j=1, \ldots, J,
\end{aligned}
$$

Unless otherwise noted, the solutions derived in the remainder of this chapter use the neutron continuity notation, but the principles are applicable to the full neutron transport equation.

For some applications, delayed neutrons are unimportant to the solution. In this case, the delayed fission term replaces the precursor term in Eq. (2.10) and is grouped 
with the prompt fission term, yielding

$$
\frac{1}{v} \frac{\partial \phi}{\partial t}+\mathbf{M} \phi(\mathbf{r}, E, t)=\mathbf{F} \phi+S(\mathbf{r}, E, t)
$$

where $\mathbf{F}$ is the combined fission operator

$$
\mathbf{F} \psi(\mathbf{r}, E, \hat{\mathbf{\Omega}}, t)=\iint \frac{\chi}{4 \pi} \bar{\nu} \Sigma_{f} \psi^{\prime} d E^{\prime} d \mathbf{\Omega}^{\prime}
$$

For steady-state problems, the time derivative term is zero, and the time variable is eliminated from Eq. (2.12). This section provides overviews on methods for solving the time-dependent set of equations in Eqs. (2.10) and (2.11) [8].

\subsubsection{Direct Methods}

The most direct way for solving Eqs. (2.10) and (2.11) is to discretize the positionenergy-time phase space. First, divide the energy space to form the multigroup diffusion equations. With the finite difference method, divide the position space into a set of finite volumes $i, j, k$. Then, formulate a set of spatially-discretized equations for the time-dependence of the scalar fluxes $\phi_{g}^{i, j, k}(t)$ and precursor densities $C_{l}^{i, j, k}(t)$, and advance over time steps to generate time-dependent solutions [9],

$$
\frac{\partial \phi_{g}^{i, j, k}(t)}{\partial t}=\frac{\phi_{g}^{i, j, k}\left(t_{n+1}\right)-\phi_{g}^{i, j, k}\left(t_{n}\right)}{t_{n+1}-t_{n}} .
$$

Solving the time-dependent multigroup diffusion equations in this way is possible, but impractical. For large, three-dimensional problems, the spatial mesh is often impossibly large. Coarse-mesh or nodal methods simplify computational tasks, but introduce other approximations to the solution [10]. On top of the spatial and energy discretizations, a large number of very small time steps is required to capture the appropriate flux behavior: time steps must be small enough to capture prompt fission neutron effects, on the order of $\mu \mathrm{s}$, and the number of time steps must be large enough to insure that the delayed neutron effects are captured, on the order of seconds to minutes. For direct methods, including angular dependences for large problems is difficult.

Due to these high computational costs, direct methods are normally reserved for reference calculations [9] or finding solutions for transport in simplified problems [11]; many applications prefer space-time factorization methods over these direct solutions. More elegant analytical methods exist $[12,13]$, but apply only to simple infinite or 
one-dimensional media.

\subsubsection{Space-Time Factorization Methods}

Space-time factorization is a blanket term for several methods built upon splitting the time-dependent neutron flux into an amplitude and shape function [14],

$$
\phi(\mathbf{r}, E, t)=p(t) \cdot \psi(\mathbf{r}, E, t)
$$

with the additional requirement that

$$
\int_{V} \int_{0}^{\infty} \frac{w(\mathbf{r}, E) \psi(\mathbf{r}, E, t)}{v(E)} d E d V=\text { constant }
$$

where $w(\mathbf{r}, E)$ is an arbitrary weight function. This requirement makes the flux factorization unique and constrains the time-variation of the shape function $\psi(\mathbf{r}, E, t)$. The effect of this constraint is that the major time dependence shifts to the amplitude function $p(t)$. Most often, the weight function is taken to be the solution of the static adjoint problem for the initial state $\phi_{0}^{\dagger}(\mathbf{r}, E)$, because it fulfils the requirements for this condition,

$$
\int_{V} \int_{0}^{\infty} \frac{\phi_{0}^{\dagger}(\mathbf{r}, E) \psi(\mathbf{r}, E, t)}{v(E)} d E d V=K_{0}
$$

Splitting the neutron flux into two functions leads to the reformulation of Eqs. (2.10) and (2.11) into equations for the shape and amplitude functions [10]. Inserting the factorization into Eq. (2.10) yields

$$
\frac{1}{v} \frac{\partial p(t) \psi(\mathbf{r}, E, t)}{\partial t}+\mathbf{M} p \psi=\mathbf{F}_{\mathbf{p}} p \psi+\sum_{j} \chi_{j} \lambda_{j} C_{j}+S
$$

Performing the chain rule on the first term, rearranging the result, and dividing by the amplitude function yields the shape equation,

$$
\left[-\mathbf{M}+\mathbf{F}_{\mathbf{p}}\right] \psi+\frac{1}{p} \sum_{j} \frac{\chi_{j}}{4 \pi} \lambda_{j} C_{j}+\frac{1}{p} S=\frac{1}{v}\left[\frac{d p(t)}{d t} \frac{\psi}{p}+\frac{\partial \psi(\mathbf{r}, E, t)}{\partial t}\right]
$$

This yields the shape function if the amplitude function is known. Derivation of the amplitude equations requires a few more steps: (i) add and subtract the total quasi-static delayed neutron source $\sum_{j} \chi_{j} \mathbf{F}_{\mathbf{d} j}$ from the right hand side of Eq. (2.10) to form the total fission operator $\mathbf{F}$; (ii) insert the flux factorization into the result and Eq. (2.11), and for the former, perform the chain rule on the time derivative; (iii) 
multiply the resulting two equations by the solution to the static adjoint problem, multiply the precursor equation by the precursor group $j$ emission spectrum $\chi_{j}$, and integrate both over all space $\iint(\cdot) d E d V$; (iv) divide the continuity equation by the importance-weighted quasi-stationary source of fission neutrons as produced by the shape function, $F(t) ;(\mathrm{v})$ divide the precursor equation by the constant $K_{0}$; and (vi) rearrange terms and make definitions for the integral parameters. This yields equations for the amplitude $p(t)$ and neutron precursor concentrations $c_{k}(t)$ known as the exact point kinetics (EPK) equations

$$
\begin{aligned}
\frac{d p(t)}{d t} & =\frac{\rho(t)-\bar{\beta}(t)}{\Lambda(t)} p(t)+\sum_{k} \lambda_{k} c_{k}(t)+s(t), \\
\frac{d c_{k}(t)}{d t} & =-\lambda_{k} c_{k}(t)+\frac{\bar{\beta}_{k}(t)}{\Lambda(t)} p(t),
\end{aligned}
$$

with the definitions

$$
\begin{aligned}
\rho(t) & =\frac{1}{F(t)} \int_{V} \int_{0}^{\infty} \phi_{0}^{\dagger}(\mathbf{r}, E)(-\mathbf{M}+\mathbf{F}) \psi(\mathbf{r}, E, t) d E d V, \\
c_{k}(t) & =\frac{1}{F(t) \Lambda(t)} \int_{V} \int_{0}^{\infty} \phi_{0}^{\dagger}(\mathbf{r}, E) \chi_{j}(E) C_{j}(\mathbf{r}, t) d E d V, \\
\bar{\beta}_{k}(t) & =\frac{1}{F(t)} \int_{V} \int_{0}^{\infty} \phi_{0}^{\dagger}(\mathbf{r}, E) \chi_{j} \mathbf{F}_{\mathbf{d} j} \psi(\mathbf{r}, E, t) d E d V, \\
\bar{\beta}(t) & =\sum_{k} \beta_{k}(t), \\
\Lambda(t) & =\frac{K_{0}}{F(t)}, \\
s(t) & =\frac{1}{F(t) \Lambda(t)} \int_{V} \int_{0}^{\infty} \phi_{0}^{\dagger}(\mathbf{r}, E) S(\mathbf{r}, E, t) d E d V, \\
F(t) & =\int_{V} \int_{0}^{\infty} \phi_{0}^{\dagger}(\mathbf{r}, E) \mathbf{F} \psi(\mathbf{r}, E, t) d E d V,
\end{aligned}
$$

for the kinetic parameters: reactivity $\rho(t)$, group $k$ effective delayed neutron fractions $\bar{\beta}_{k}$, and prompt neutron lifetime $\Lambda(t)$. This factorization approach does not introduce an approximation [3].

Together, the EPK and shape equations form a coupled set of nonlinear equations: the solution of Eq. (2.19) implies that the amplitude function is known, and the kinetic parameters in Eqs. (2.20) and (2.21) require the shape function. These equations make up the basis for space-time factorization methods. The straightforward solution to these equations is an iterative procedure that calculates the amplitude function for 
small time steps $\delta t$ and updates the shape function for larger time steps $\Delta t$. The ratio of the time steps $\Delta t / \delta t$ is large for ideal problems where the shape function has little time variation. But, for some transients, like those in a loosely coupled reactor, the ratio is smaller due to persistent flux distortions [15].

The method for updating the shape function distinguishes the different spacetime factorization methods. With a reduction in the complexity of these methods, the accuracy of the solutions decrease and computation eases. Quasi-static Methods (QM) have the most sophisticated approach in calculating the shape functions. The Predictor-Corrector QM (PCQM) uses the EPK equations and Eqs. (2.10) and (2.11), excluding the shape equation from the iteration. Shape functions obtained from the original continuity and precursor equations provide better kinetic parameters, and the PCQM shows improvements over other QM [15]. The Improved QM (IQM) uses the EPK and shape equations, but approximates the derivative term in Eq. (2.19) by backward differencing,

$$
\frac{\partial}{\partial t} \psi(\mathbf{r}, E, t)=\frac{[\psi(\mathbf{r}, E, t)-\psi(\mathbf{r}, E, t-\Delta t)]}{\Delta t},
$$

chosen for its simplicity and stability with a large step length [16]. This is the most common method used in spatial kinetics codes [10]. The original Quasi-static Method (QM) differs from the IQM in that it excludes the time derivative term in Eq. (2.19) entirely, citing that the time variation of the shape function is of much less importance than that of the amplitude function [14]. The Adiabatic Method uses shape functions that are the solution to the steady-state continuity equation computed at different times during the transient. This effectively negates the right-hand side of Eq. (2.19) as well as groups the precursor term into the fission operator [16]. Finally, the most simplified method assumes complete separability of time from the rest of the phase space,

$$
\phi(\mathbf{r}, E, t)=p(t) \cdot \psi(\mathbf{r}, E)
$$

This assumption leads to the reformulation of the EPK equations into the point kinetics $(\mathrm{PK})$ equations,

$$
\begin{aligned}
\frac{d p(t)}{d t} & =\frac{\rho(t)-\bar{\beta}}{\Lambda} p(t)+\sum_{k} \lambda_{k} c_{k}(t)+s(t), \\
\frac{d c_{k}(t)}{d t} & =-\lambda_{k} c_{k}(t)+\frac{\bar{\beta}_{k}}{\Lambda} p(t),
\end{aligned}
$$

where the initial flux shape $\phi_{0}(\mathbf{r}, E)$ replaces the time-dependent shape function in 
the calculation of the point kinetics parameters [3]. A quick comparison of these space-time factorization methods (Table 2.2) shows the differences in updating the shape function.

Table 2.2: A summary of calculation procedures for space-time factorization methods.

\begin{tabular}{llll}
\hline Method & $\begin{array}{l}\text { Amplitude function } \\
\text { calculation }\end{array}$ & Shape function calculation & Refs. \\
\hline $\begin{array}{l}\text { Predictor-Corrected } \\
\text { Quasi-static }\end{array}$ & EPK Eqs. & $\begin{array}{l}\text { Neutron continuity and } \\
\text { precursor equations }\end{array}$ & {$[15]$} \\
$\begin{array}{l}\text { Improved } \\
\text { Quasi-static }\end{array}$ & EPK Eqs. & $\begin{array}{l}\text { Shape equation with } \\
\text { backward differencing for } \\
\frac{\partial}{\partial t} \psi(\mathbf{r}, E, t)\end{array}$ & {$[16,15,10]$} \\
Quasi-static & EPK Eqs. & $\begin{array}{l}\text { Shape equation excluding } \\
\text { St } \psi(\mathbf{r}, E, t)\end{array}$ & {$[14,16,10]$} \\
& & $\begin{array}{l}\text { Steady-state eigenvalue } \\
\text { problem }\end{array}$ & {$[16]$} \\
Adiabatic & EPK Eqs. & Initial flux shape $\phi_{0}(\mathbf{r}, E)$ & {$[3,10]$} \\
& & & \\
\hline
\end{tabular}

\subsubsection{Expansion Methods}

Expansion methods include both synthesis and modal methods. These methods expand the time-dependent flux into a linear combination of a time function $T_{i}(t)$ multiplied by a shape function $\phi_{i}(\mathbf{r}, E)$,

$$
\phi(\mathbf{r}, E, t)=\sum_{i=0}^{N} T_{i}(t) \phi_{i}(\mathbf{r}, E)
$$

where $N$ is the desired number of summation elements. While the $i$ th element of the summation in Eq. (2.33) represents a complete space-time separation, the summed result of these functions is inseparable in space and time.

The difference between synthesis and modal methods is the shape functions $\phi_{i}(\mathbf{r}, E)$, as both use similar procedures in determining the expansion coefficients and time function [10]. For synthesis methods, the shape functions are solutions to steady-state problems for two or more conditions expected during the transient. A good choice of these functions requires judgement and intuition. Synthesis methods have good accuracy and flexibility, but they also encounter some numerical difficulties in calculating the expansion coefficients and lack of meaningful error bounds [17]. For 
modal methods, the shape functions are the eigenfunctions from one of the several eigenvalue problems of the neutron continuity and precursor equations. Thus, this method is also known as eigenfunction expansion. For time-dependent problems, the $\alpha$ eigenfunctions yield the best solutions for the space-time dependent flux. Each $\alpha$ eigenfunction describes a unique spatial flux dependence while the $\alpha$ eigenvalues describe the time behavior of that unique eigenfunction. The adjoint eigenfunctions are also necessary for calculating expansion coefficients. The next section provides an in-depth discussion on the $\alpha$ eigenvalue problem and eigenfunction expansion.

With the proper shape functions, synthesis methods offer better accuracy than other factorization methods [18], while needing fewer expansion functions than modal methods. The disadvantage of synthesis methods is the need to choose good shape functions to insure the summed result is non-negative. For modal methods, a large

number of modes is necessary for sufficient accuracy on the time scales of $\mu \mathrm{s}$ [9]. For these reasons, modal methods are seen as prohibitively costly, and together, synthesis and modal methods have been largely abandoned for the purpose of solving kinetics problems [10].

\subsubsection{Time-Dependent Monte Carlo}

The previous sections discuss deterministic methods for solving time-dependent problems. Time-dependent Monte Carlo (TDMC) is an extension of the Monte Carlo method described in $\S 1.2$. By directly simulating neutron histories and tracking the time of events, TDMC generates solutions with some associated statistical error. With each neutron individually tracked, TDMC is a natural extension of steady-state Monte Carlo, with three additional concepts to discuss: tracking time, censusing, and delayed neutron emission.

\section{The Monte Carlo Method}

The following is a simple example of the simulation of a single history with the Monte Carlo method. First, generate initial information about the neutron from an appropriate source distribution: position, direction of travel, energy, and occasionally time. Then, determine the distance to collision with the random number $\xi_{1}$. The probability $p(x) d x$ of a first collision between $x$ and $x+d x$ is

$$
p(x) d x=\exp \left(-\Sigma_{t} x\right) \Sigma_{t} d x
$$


where $\Sigma_{t}$ is the total macroscopic cross section of the medium and $p(x)$ is known as the probability density function (PDF) [19]. For a continuous PDF $p(t)$, the integral

$$
\xi=\int_{0}^{x} p(t) d t
$$

determines $x$ uniquely as a function of $\xi$. Integration of the PDF in Eq. (2.34) as shown in Eq. (2.35) and rearranging terms yields

$$
x=-\frac{1}{\Sigma_{t}} \ln \left(1-\xi_{1}\right)
$$

Due to the uniform sampling of $\xi \in(0,1)$, the quantity $1-\xi$ is also uniformly sampled $\in(0,1)$. The distance to collision simplifies to

$$
x=-\frac{1}{\Sigma_{t}} \ln \xi_{1}
$$

Next, determine the nuclide with which the neutron collides using the next random number $\xi_{2}$. A given material is usually made up of several nuclides, and the total macroscopic cross sections of each determine the interaction probabilities. The probability of interaction with nuclide $i$ is

$$
p_{i}=\frac{\Sigma_{t i}}{\sum_{j=1}^{N} \Sigma_{t j}}=\frac{\Sigma_{t i}}{\Sigma_{t}}
$$

where $N$ is the total number of nuclides in the material. Comparing the random number $\xi_{2}$ to the probabilities, the collision nuclide $i$ is that for which

$$
\sum_{j=1}^{i-1} p_{j} \leq \xi_{2}<\sum_{j=1}^{i} p_{j}
$$

is true. Next, determine the collision type with the next random number $\xi_{3}$. The cross sections of each collision type determine the probabilities; e.g., the radiative capture probability is

$$
p_{\gamma}=\frac{\Sigma_{\gamma i}}{\Sigma_{t i}}
$$

where $\Sigma_{\gamma i}$ is the capture cross section of nuclide $i$. Comparison of the random number $\xi_{3}$ to the probabilities as in Eq. (2.39) yields the collision type. Next, take the appropriate action according to the collision type. If the nuclide absorbs the neutron, the history ends. If the neutron scatters off the nuclide, perform the kinematics and 
emit the neutron at a new energy and direction.

This short example of a single neutron event only uses a few random numbers $\xi$, but in some materials, neutrons undergo many collision events before being destroyed. Also, Monte Carlo methods use random numbers in determining the source characteristics and reemission energy and direction after a neutron collision. Monte Carlo accounts for the problem geometry while determining the distance to next collision. A sequence of particle events is known as a random walk.

While simulating neutron histories, Monte Carlo tallies extract desired information. Tallies accumulate scores $x_{n}$ for specific events during each neutron history, such as collisions with a given nuclide, crossing specific boundaries, or distance traveled in a given volume. After the simulation, dividing the scores by the total number of neutron histories $N$ yields average probabilities $\bar{x}$ of given events [6],

$$
\bar{x}=\frac{1}{N} \sum_{n=1}^{N} x_{n} .
$$

Common types of Monte Carlo tallies include volume-averaged flux in a region, fission heating, and leakage rates. Criticality calculations also use Monte Carlo tallies to compute the multiplication of neutrons in a problem with fissile material.

With a very large number of particle histories, often millions or more, the averaged results in Eq. (2.41) are close to the true solution. With accurate cross sections and a detailed geometry, Monte Carlo solutions avoid truncation errors, but have random statistical errors that trend to zero in the limit of an infinite number of particle histories. In practice, Monte Carlo simulations have a finite number of histories $N$, leaving an associated statistical error in extracted results. For very large $N$, the statistical error $\epsilon$ in some estimated average quantity is a function of the standard deviation $\sigma$ of the quantity and the number of particle histories [20],

$$
\epsilon \approx \frac{\sigma}{\sqrt{N}}
$$

To decrease $\epsilon$, either reduce $\sigma$ or increase $N$. Methods of reducing $\sigma$ are known as variance reduction techniques, such as implicit capture and geometry splitting with Russian roulette. Overall, the statistical error decreases relatively slowly, as $1 / \sqrt{N}$. This slow convergence often limits Monte Carlo methods to solving steadystate problems, and though time-dependent Monte Carlo was conceptualized for the first Monte Carlo codes, its application to reactor transients has only been studied in more recent years [21]. 
Many variance reduction techniques increase runtime, trading time for accuracy. A useful parameter for quantifying this tradeoff is the Figure of Merit (FOM) [6]

$$
F O M=\frac{1}{\sigma^{2} T}
$$

where $T$ is the mean time per history. The FOM determines the gain in accuracy for a given computation time, with a larger FOM identifying a advantageous technique. Some techniques actually cause an increase in $\sigma^{2}$, but reduce computation time, resulting in a larger FOM.

\section{Additional Considerations for Tracking Time Dependence}

Along with neutron position, direction, and energy, TDMC keeps track of an additional variable: time. As with the other variables, TDMC treats time as continuous. Consider a neutron with time $t=t_{0}$ and energy $E$. After sampling the distance to collision, TDMC calculates the neutron speed,

$$
v=\sqrt{\frac{2 E}{m_{n}}},
$$

where $m_{n}$ is the mass of a neutron, and calculates the time to collision,

$$
\Delta t=\frac{x}{v}=-\sqrt{\frac{m_{n}}{2 E}} \frac{1}{\Sigma_{t}} \ln \xi
$$

where $x$ is the distance to next collision sampled from random number $\xi$ and the total macroscopic cross section $\Sigma_{t}$. Finally, TDMC advances the neutron time,

$$
t=t_{0}+\Delta t
$$

and performs the appropriate actions for the collision type. For collisions and prompt fission, TDMC assumes the time between the start of the collision and neutron reemission is zero because it is so small. For delayed neutron emission, TDMC samples an emission time from the decay constant of the appropriate precursor group. The initial time of the neutron $t_{0}$ depends on the source time characteristics.

Though TDMC treats the time space as continuous, its extracts information at specific times or within time intervals. One way of extracting fluxes or reaction rates is to divide the time space into small increments and perform tallies on neutrons that fall in each of these time increments. This is similar to how a multichannel 
analyzer works for recording detector response: it registers total counts in a given time interval for each channel. Another way is to extract the distribution of the flux at an exact point in time with censusing. This involves using specific times $t_{c}$ as boundaries. When a neutron crosses this boundary, i.e., $t>t_{c}$, TDMC stores the neutron position, direction, and energy at $t=t_{c}$ in a bank. After all $N$ neutrons reach this boundary, the next cycle begins by emitting and tracking the neutrons from the bank, and the sequence repeats until neutrons reach the last census. With additional techniques to ensure enough neutrons survive to the next census, this method greatly increases the number of neutrons reaching each boundary, improving confidence in statistical solutions.

The treatment of delayed neutron emission poses potential statistical troubles for TDMC, due to low emission probability of less than $1 \%$ and the number of groups representing delayed neutron precursors. These factors lead to low probabilities for delayed neutron emission during a Monte Carlo run. A direct treatment of delayed neutron emission generates an insufficient delayed neutron source, and for many transients, this causes significant errors in the solution. For example, consider a problem where the desired solution is the time-dependent flux up to a few seconds. Then, sampling a delayed neutron after this time contributes nothing to the solution. A remedy for this is restricting the sampling of emission time to the window of interest, and applying proper weights to correct for any overestimation in the delayed neutron source. An extension of this is forcing precursors to decay over every time interval of interest [21]. These approaches provide better statistics for the delayed neutron source, which only becomes more difficult to characterize with the newest delayed neutron data $[22]$.

For transients in simple systems, TDMC demonstrates good accuracy in calculating the dynamic behavior of the flux [23]. But, the slow decrease of statistical errors in Monte Carlo limits the applicability of TDMC to simple problems: the number of neutron histories required for converged solutions of transients in large, three-dimensional problems is prohibitively large. Some Monte Carlo calculations provide kinetic parameters useful for characterizing time-dependent problems, such as the prompt neutron lifetime [6].

\subsection{The $\alpha$ Eigenvalue}

For most systems with or without fissile material, there exists a constant or set of constants $\alpha$ that represent the exponential time-dependent behavior of the neutron 
flux [24]. These constants are the eigenvalues of the linear or neutron transport operator and are known as $\alpha$ eigenvalues [4], and have units inverse time. In some literature, these are known as $\lambda[2]$, natural [10], or time [25] eigenvalues. The $\alpha$ eigenvalues identify the condition of a system and the $\alpha$ eigenfunctions $\phi_{\alpha}$ describe the phase space dependence for obtaining time-dependent solutions via modal methods. Together, an eigenvalue and its eigenfunction are known as an eigenpair.

\subsubsection{The Linear Transport Operator}

Formulating the $\alpha$ eigenvalue problem begins with the separation of the timedependent neutron flux and precursor concentrations into shape functions and time functions [1]. Describing the time function as an exponential yields

$$
\begin{aligned}
\phi(\mathbf{r}, E, t) & =\phi(\mathbf{r}, E) \exp (\alpha t), \\
C_{j}(\mathbf{r}, t) & =C_{j}(\mathbf{r}) \exp (\alpha t), \quad \text { for } \quad j=1, \ldots, J
\end{aligned}
$$

whose derivatives with respect to time are the those of the exponential function. Inserting these factorizations and their derivatives into Eqs. (2.10) and (2.11), excluding the external source, and dividing out the exponential yields

$$
\begin{aligned}
\frac{\alpha}{v} \phi+\mathbf{M} \phi(\mathbf{r}, E) & =\mathbf{F}_{\mathbf{p}} \phi+\sum_{j}^{J} \chi_{j} \lambda_{j} C_{j}, \\
\alpha C_{j}+\lambda_{j} C_{j}(\mathbf{r}) & =\mathbf{F}_{\mathbf{d} j} \phi, \quad \text { for } \quad j=1, \ldots, J
\end{aligned}
$$

the full $\alpha$-eigenvalue problem. These equations are similar to the steady-state equations except for the additional terms containing the $\alpha$ eigenvalue. The $\alpha / v$ term is known as the time-absorption term because it augments the collision cross section in the operator M. Similarly, the $\alpha$ term in Eq. (2.50) augments the decay constant $\lambda_{j}$. For some problems, delayed neutron emission is unimportant. Ignoring the delayed neutron precursors yields

$$
\frac{\alpha}{v} \phi+\mathbf{M} \phi(\mathbf{r}, E)=\mathbf{F}_{\mathbf{p}} \phi
$$

the prompt $\alpha$-eigenvalue problem. The solutions of Eq. (2.51) are known as prompt eigenvalues $\alpha_{p}$ and eigenfunctions.

It is sometimes more convenient to view the eigenvalue problems in matrix form. 
Rearranging the terms in Eqs. (2.49) and (2.50) yields

$$
\left[\begin{array}{ll}
v & 0 \\
0 & I
\end{array}\right]\left[\begin{array}{cc}
-\mathbf{M}+\mathbf{F}_{\mathbf{p}} & \boldsymbol{\chi} \boldsymbol{\lambda} \\
\mathbf{F}_{\mathbf{d}} & -\boldsymbol{\lambda}
\end{array}\right]\left[\begin{array}{c}
\phi \\
\boldsymbol{C}
\end{array}\right]=\alpha\left[\begin{array}{c}
\phi(\mathbf{r}, E) \\
\boldsymbol{C}(\mathbf{r})
\end{array}\right]
$$

the matrix form of the full $\alpha$-eigenvalue problem where the left matrix is the speed matrix and $I$ is the identity matrix. Additional definitions simplify the inclusion of $J$ precursor groups in the matrix formulation: $\boldsymbol{C}$ is a length $J$ column vector of the precursor group concentrations $C_{j}, \mathbf{F}_{\mathbf{d}}$ is a length $J$ column vector of group delayed fission operators $\mathbf{F}_{\mathbf{d} j}, \boldsymbol{\chi} \boldsymbol{\lambda}$ is a length $J$ row vector of the emission spectrum multiplied by the decay constant $\chi_{j} \lambda_{j}$, and $\boldsymbol{\lambda}$ is a size $J \times J$ diagonal matrix with decay constants $\lambda_{j}$ on the diagonal. The combination of the two matrices on the left hand side of this equation is the linear transport operator

$$
\mathbf{A}(\mathbf{r}, E)=\left[\begin{array}{ll}
v & 0 \\
0 & I
\end{array}\right]\left[\begin{array}{cc}
-\mathbf{M}+\mathbf{F}_{\mathbf{p}} & \chi \boldsymbol{\lambda} \\
\mathbf{F}_{\mathbf{d}} & -\boldsymbol{\lambda}
\end{array}\right]
$$

Likewise, rearranging terms in Eq. (2.51) yields the prompt matrix problem

$$
v\left[-\mathbf{M}+\mathbf{F}_{\mathbf{p}}\right] \phi=\alpha_{p} \phi(\mathbf{r}, E)
$$

where the linear transport operator excluding delayed neutron precursors is

$$
\mathbf{A}_{\mathbf{p}}(\mathbf{r}, E)=v\left[-\mathbf{M}+\mathbf{F}_{\mathbf{p}}\right]
$$

\subsubsection{Relation to Other Eigenvalue Problems}

Knowing the relationship between the $\alpha$ and other eigenvalues of the neutron transport equation aids in the discussion of the behavior of the $\alpha$-eigenvalue spectrum and the identification of system criticality. The $k, c$, and $\delta$ eigenvalues represent the effective fission multiplication factor, effective collision multiplication factor, and effective density factor, respectively [26]. Unlike $\alpha$, these eigenvalues are dimensionless and represent an imbalance between specific terms of the steady-state neutron transport equation [27]. The $\delta$ eigenvalue is relatively obscure and is left from the discussion. The TRMM uses the $k$ and $c$ eigenvalues in calculating transition rates in multiplying and non-multiplying media, respectively. Monte Carlo codes calculate these eigenvalues with a power iteration method [28]. 


\section{The $k$ Eigenvalue}

The $k$ eigenvalue represents the ratio between the fission source and losses due to collision and leakage. It has the additional physical interpretation as the effective neutron multiplication factor per neutron generation: it is the ratio of neutrons in the next generation to those in the current generation [29]. Due to this interpretation, the $k$ eigenvalue is a measure of the criticality of the system.

To obtain the $k$-eigenvalue problem, scale the fission operator in Eq. (2.12) by $1 / k$. Eliminating the time dependent term and assuming no external source yields

$$
\mathbf{M} \phi(\mathbf{r}, E)=\frac{1}{k} \mathbf{F} \phi
$$

the $k$-eigenvalue problem [1]. This effectively varies the number of neutrons emitted per fission by the energy-independent factor of $1 / k$ in order to achieve neutron balance.

For any multiplying system, there exists a unique positive $k$ eigenvalue corresponding to an all-real and non-negative eigenfunction $\phi_{k}(\mathbf{r}, E)$. It is the largest eigenvalue that satisfies Eq. (2.56), $k_{0}$ or $k_{\text {eff }}$, and it fulfills the physical interpretation of the $k$ eigenvalue as a measure of criticality. The criticality of a system defines the behavior of the neutron population in the absence of an external source: in a critical system, the neutron population remains unchanged with time; in a subcritical system, the neutron population decreases with time; and in a supercritical system, the neutron population increases with time [2]. The $k_{0}$ determines the condition by following the trend:

$$
k_{0}\left\{\begin{array}{lll}
>1 & \text { if } & \text { supercritical } \\
=1 & \text { if } & \text { critical } \\
<1 & \text { if } & \text { subcritical. }
\end{array}\right.
$$

For a non-multiplying system, $k_{0}=0$. Numerical criticality searches determine the $k_{0}$ of a given problem.

Solutions to the $k$-eigenvalue problem are useful for reactor physics applications because much of the analysis is done for systems designed to be critical, $k_{0}=1$. At critical, the eigenfunction corresponding to the $k_{0}$ of the system is the flux shape within the reactor, and near-critical, this eigenfunction is a good approximation of this shape. This is somewhat counterintuitive because if $k_{0}<1$ or $k_{0}>1$, the neutron population changes with time. But, the calculation of $k_{0}$ balances Eq. (2.56), yielding an eigenfunction calculated at a system condition that has been made artificially critical using the $1 / k$ factor. While this hardens or softens the energy spectrum in 
subcritical or supercritical systems [26], it still serves as a suitable approximation of the steady-state flux.

The other $k$ eigenvalues that satisfy Eq. (2.56) are known as higher $k$ eigenvalues and together with $k_{0}$ make up the $k$-eigenvalue spectrum

$$
k_{0}>k_{1}>k_{2}>\cdots k_{i}
$$

where the $k_{i}$ are real and positive. While the physical meaning of $k_{0}$ and $\phi_{0}$ makes them useful for reactor design, the higher $k$ eigenpairs have more indirect uses. The dominance ratio, the ratio $k_{1} / k_{0}$, provides some measure of numerical convergence [30]. Some other applications of higher $k$ eigenvalues include perturbation theory or calculating flux tilts in loosely-coupled cores [31]. Many of these applications only need a few of the next higher eigenpairs.

For simple problems, the entire $k$-eigenvalue spectrum is readily obtainable. But, for more complex problems, finding many higher $k$ eigenpairs is more difficult and often carried out using iterative methods [30]. Recent research in approximating $k$ eigenpairs with matrix methods similar to the TRMM show that realistic problems have a set of positive, real $k$ eigenvalues as expected [32].

The $\alpha$ - and $k$-eigenvalue problems are equal for an exactly critical system [27]. In this case, $k_{0}=1$ and $\alpha_{0}=0$, and the eigenfunctions $\phi_{k}$ and $\phi_{\alpha}$ corresponding to these eigenvalues are equal. Still, both problems contain a separate set of higher eigenvalues. For an infinite medium, the one-group $k$ - and $\alpha$-eigenvalue problems are

$$
\begin{aligned}
\Sigma_{t} \phi & =\Sigma_{s} \phi+\frac{\bar{\nu} \Sigma_{f}}{k_{\infty}} \phi, \\
\frac{\alpha_{\infty}}{v} \phi & =\Sigma_{s} \phi+\bar{\nu} \Sigma_{f} \phi-\Sigma_{t} \phi .
\end{aligned}
$$

Dividing out the fluxes and combining these two equations yields a relationship between the two infinite eigenvalues [33]

$$
\frac{\alpha_{\infty}}{v \Sigma_{t}}=\left(k_{\infty}-1\right)\left(1-\frac{\Sigma_{s}}{\Sigma_{t}}\right) .
$$

A Monte Carlo $k$-eigenvalue calculation begins with initial guesses for the eigenvalue $k_{0}^{(0)}$ and a source. With these guesses, Monte Carlo generates particle histories to solve for $\phi_{k}^{(1)}$ using the $k$-eigenvalue problem for iteration $n$,

$$
\mathbf{M} \phi_{k}^{(n+1)}=\frac{1}{k_{0}^{(n)}} \mathbf{F} \phi_{k}^{(n)}
$$


a reformulation of Eq. (2.56) for updating $\phi_{k}$. During the random walk, Monte Carlo estimates $k_{0}^{(1)}$ by tracking the neutrons emerging in the next generation. Rearranging Eq. (2.56), multiplying by the fission operator $\mathbf{F}$, integrating over all space $\int_{V}(\cdot) d V$, and solving for $k$ yields

$$
k_{0}^{(n+1)}=\frac{\int_{V} \mathbf{F M}^{-1} \mathbf{F} \phi_{k}^{(n)} d V}{\int_{V} \mathbf{F} \phi_{k}^{(n)} d V},
$$

where the superscripts are introduced to estimate the $k$ at the end of the current generation. Performing the same operations on Eq. (2.62) and combining these two results yields an equation to update $k_{0}$

$$
k_{0}^{(n+1)}=k_{0}^{(n)} \frac{\int_{V} \mathbf{F} \phi_{k}^{(n+1)} d V}{\int_{V} \mathbf{F} \phi_{k}^{(n)} d V},
$$

which is the ratio of the old and new fission sources. With the new estimates $k_{0}^{(1)}$ and $\phi_{k}^{(1)}$, Monte Carlo generates a new set of particle histories and the process repeats until $k_{0}^{(n)}$ and $\phi_{k}^{(n)}$ satisfy desired convergence criteria [28]. At this point, Monte Carlo continues to run particle histories to extract meaningful tallies.

Each iteration is known as a batch and the number of particle histories per iteration $N$ is referred to as the batch size. During each batch, Monte Carlo stores the fission sites and uses them as source points for the next batch: each batch is a fixedsource calculation where the source is the initial guess or updated fission source. At a fission site, Monte Carlo stores a number of neutrons depending on the probability of fission at that location

$$
\left\lfloor\frac{W \bar{\nu} \sigma_{f}}{\sigma_{t} k_{0}^{(n)}}+\xi\right\rfloor,
$$

where $W$ is the particle weight, $\sigma_{f}$ and $\sigma_{t}$ are the fission and total microscopic cross sections, and $\xi$ is a random number $\in(0,1)$. Note that this quantity is floored to obtain an integer. Collision, absorption, and track length tally estimators provide 
alternatives to Eq. (2.64) for obtaining values for $k_{0}^{(n+1)}$ during the random walk:

$$
\begin{aligned}
k_{0}^{C} & =\frac{1}{N} \sum_{i}^{\text {collisions }} W_{i}\left(\frac{\sum_{j} f_{j} \bar{\nu}_{j} \sigma_{f_{j}}}{\sum_{j} f_{j} \sigma_{t_{j}}}\right), \\
k_{0}^{A} & =\frac{1}{N} \sum_{i}^{\text {absorptions }} W_{i} \bar{\nu}_{j}\left(\frac{\sigma_{f_{j}}}{\sigma_{c_{j}}+\sigma_{f_{j}}}\right), \\
k_{0}^{T L} & =\frac{1}{N} \sum_{i}^{\text {trajectories }} W_{i} \rho d \sum_{j} f_{j} \bar{\nu}_{j} \sigma_{f_{j}},
\end{aligned}
$$

where ${ }_{j}$ designates the nuclides of the material, $f$ is the atomic fraction of a nuclide, $\sigma_{c}$ is the capture cross section, $\rho$ is the atomic density of the material, and $d$ is the trajectory track length from the last event [34].

\section{The c Eigenvalue}

The $c$ eigenvalue represents the ratio of the combined fission and inscattering source to losses due to collisions and leakage. It has the physical interpretation as the number of secondary neutrons, or the multiplication factor, per collision event. In some literature it is denoted as $\lambda[27]$ or $\gamma[29]$.

Formulation of the $c$-eigenvalue problem requires the operator $\mathbf{M}$ in Eq. (2.12) be split into the operators $\mathbf{L}, \mathbf{R}$, and $\mathbf{S}$. Scaling the combined inscatter and fission operators by $1 / c$, eliminating the time dependent term, and assuming no external source yields

$$
[\mathbf{L}+\mathbf{R}] \phi(\mathbf{r}, E)=\frac{1}{c}[\mathbf{S}+\mathbf{F}] \phi,
$$

the $c$-eigenvalue problem. This effectively varies the number of neutrons exiting collision events by the factor $1 / c$. The TRMM uses the collision eigenvalue for nonmultiplying media because the $k$ eigenvalue is zero; in this case, the $1 / c$ factor only acts upon the inscatter source term.

With respect to the $k$ eigenvalue, the $c$ eigenvalue follows the same trend in Eq. (2.57), affects the energy spectrum similarly, and exhibits similar eigenvalue spectral properties [27]. But, it varies quite differently for subcritical and supercritical media: in some problems, the $k$ eigenvalue deviates from unity more than the $c$ eigenvalue. The magnitude of the effect of the $c$ eigenvalue on the energy spectrum is suppressed because the eigenvalue is also operating upon the inscatter source, which has a softer spectrum than the fission operator. In a supercritical system, the spectrum of the $k$ solution is softer than the spectrum of the $c$ solution, and the opposite 
is true in a subcritical system. For some problems, the $c$ eigenvalue is a more efficient quantity for criticality searches [35].

The $\alpha$ - and $c$-eigenvalue problems are equal for a critical system where $\alpha_{0}=0$ and $c_{0}=1$, and the eigenfunctions $\phi_{\alpha}$ and $\phi_{c}$ corresponding to these eigenvalues are equal. In this case, the $k_{0}=1$ and its eigenfunction $\phi_{k}$ are also equal to those of the $\alpha$ - and $c$-eigenvalue problems.

A Monte Carlo c-eigenvalue calculation is similar to a $k$-eigenvalue calculation. With the initial guess $c_{0}^{(0)}$ and a source, Monte Carlo generates particle histories to solve for $\phi_{c}^{(1)}$ using the $c$-eigenvalue problem for iteration $n$,

$$
[\mathbf{L}+\mathbf{R}] \phi_{c}^{(n+1)}=\frac{1}{c_{0}^{(n)}}[\mathbf{S}+\mathbf{F}] \phi_{c}^{(n)}
$$

During the random walk, Monte Carlo estimates $c_{0}^{(1)}$ by tracking neutrons emerging from collisions. Following the same steps as to obtain Eq. (2.64) yields an equation to update $c_{0}$,

$$
c_{0}^{(n+1)}=c_{0}^{(n)} \frac{\int_{V}(\mathbf{S}+\mathbf{F}) \phi_{c}^{(n+1)} d V}{\int_{V}(\mathbf{S}+\mathbf{F}) \phi_{c}^{(n)} d V},
$$

which is the ratio of the old and new collision sources. The process repeats until $c_{0}^{(n)}$ and $\phi_{c}^{(n)}$ satisfy the desired convergence criteria.

During each batch, Monte Carlo stores collision sites and uses them as source points for the next batch. In determining the number of neutrons to store at each site, it is useful to define the secondary-production cross section as

$$
\sigma_{P}=\bar{\nu} \sigma_{f}+\sum_{x=1}^{\infty} x \sigma_{n, x n}
$$

where $x$ is the multiplicity of the reaction and $\sigma_{n, x n}$ is the multiplicity reaction cross section [35]. Then, the number of secondary neutrons produced at a given collision site is

$$
\left\lfloor\frac{W \sigma_{P}}{\sigma_{t} c_{0}^{(n)}}+\xi\right\rfloor
$$

and the collision estimator for $c_{0}^{(n+1)}$ is

$$
c_{0}=\frac{1}{N} \sum_{i}^{\text {collisions }} W_{i}\left(\frac{\sum_{k} f_{k} \sigma_{P}}{\sum_{k} f_{k} \sigma_{t_{k}}}\right) .
$$




\subsubsection{Spectrum}

The set of $\alpha$ eigenvalues that satisfy Eq. (2.52) are known as the $\alpha$-eigenvalue spectrum. The eigenvalue with the largest real part, $\alpha_{0}$, is the fundamental eigenvalue. It corresponds to the asymptotic solution of the system as $t \rightarrow \infty$ and follows the trend:

$$
\alpha_{0}\left\{\begin{array}{lll}
>0 & \text { if } & \text { supercritical, } \\
=0 & \text { if } & \text { critical, } \\
<0 & \text { if } & \text { subcritical. }
\end{array}\right.
$$

The eigenfunction corresponding to the fundamental eigenvalue, $\phi_{0}(\mathbf{r}, E)$, contains the spatial and energy dependence of the asymptotic solution [36]

$$
\phi_{\text {asym }}(\mathbf{r}, E, t) \propto \phi_{0}(\mathbf{r}, E) \exp \left(\alpha_{0} t\right)
$$

While the fundamental eigenpair is all real, any of the remaining higher $\alpha$ eigenpairs may be negative and complex. The $\alpha$ eigenvalues are ordered by their real parts

$$
\alpha_{0}>\operatorname{Re}\left(\alpha_{1}\right)>\operatorname{Re}\left(\alpha_{2}\right)>\cdots>\operatorname{Re}\left(\alpha_{m}\right)
$$

where, if a complex eigenvalue exists in the spectrum, than its complex conjugate is also a part of the spectrum. This is because the linear transport operator is all real, so its complex eigenvalues always occur in complex conjugate pairs and the eigenfunctions corresponding to pair of these eigenvalues are also complex conjugates. Consider the operator $\mathbf{A} \in \mathbb{R}$. If $\lambda \in \mathbb{C}$ is a complex eigenvalue of $\mathbf{A}$, with a non-zero eigenfunction $v \in \mathbb{C}^{n}$, by definition this means that

$$
\mathbf{A} v=\lambda v
$$

Taking the complex conjugate $(\bar{\cdot})$ of this equation yields

$$
\overline{\mathbf{A}} \bar{v}=\mathbf{A} \bar{v}=\bar{\lambda} \bar{v}
$$

Thus, if $\lambda$ and $v$ are a complex eigenpair of the matrix $\mathbf{A}$, then so is $\bar{\lambda}$ and $\bar{v}$. Most often, the highest eigenvalues have very large and negative real parts. A plot in the complex plane (Figure 2.1) is the best way of visualizing the entire $\alpha$-eigenvalue spectrum.

The existence of a fundamental eigenvalue is not guaranteed for all media, particularly optically thin slabs [33]. Some very subcritical media have no experimentally 
observable fundamental eigenvalue. Also, the existence of a sole dominant eigenvalue, one that has a greater real part than any other eigenvalue, has yet to be proven. For small one-speed slabs, the spectrum only consists of a few discrete eigenvalues, but for realistic continuous-energy problems, the spectrum is extensive and includes several features.

\section{Points, Lines, and the Continuum}

The initial study of the spectrum of linear transport operator $\mathbf{A}$ used the one-speed, one-dimensional form [37]

$$
\mathbf{A}(x, \mu)=-\mu \frac{\partial(\cdot)}{\partial x}+\frac{c}{2} \int_{-1}^{1}(\cdot) d \mu^{\prime},
$$

where

$$
c=\frac{\bar{\nu} \Sigma_{f}+\Sigma_{s}}{\Sigma_{t}}
$$

Since then, studies focus on building upon assertions gained for these simple problems, expanding the spectral analysis to multiple-speed media [38] and more general geometries [4]. The spectrum of $\mathbf{A}$ for more general problems consist of points, lines, and in some cases, a continuum (Figure 2.1).

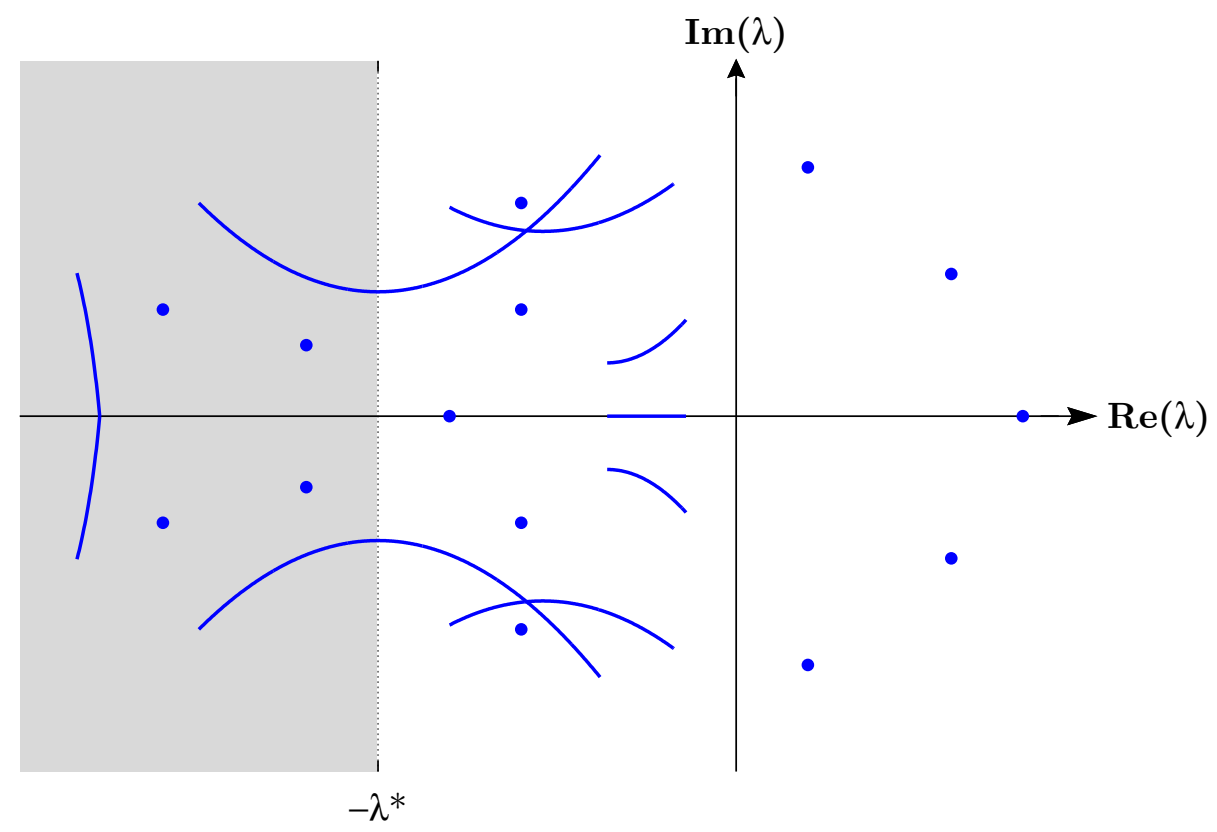

Figure 2.1: This idealized illustration of the spectrum of the linear transport operator plotted in the complex plane shows the different features present in the spectrum. 
The spectral analysis of $\mathbf{A}$ is an exercise in functional analysis. The spectrum of $\mathbf{A}$ contains three groups: continuous spectrum, residual spectrum, and point spectrum. It is important to note that this spectrum includes more scalars $\lambda$ than just the $\alpha$ eigenvalues: the $\alpha$ eigenvalues are the point and line spectrum of $\mathbf{A}$ [39]. Further discussion on spectral analysis is available in the references: this section focuses on results of this analysis, as they are important to understanding $\alpha$ eigenvalues.

The decomposition of the spectral plane is best understood for the simple operator in Eq. (2.80). In this case, there are two parts of the spectrum: point spectra and the continuum. The point spectrum is a finite, all-real set lying in the positive half-plane $\lambda>-\lambda^{*}$ (Figure 2.1), where $\lambda^{*}$ is the minimum value of $v \Sigma_{t}(v)$. The continuous spectrum contains the negative half-plane $\operatorname{Re}(\lambda) \leq-\lambda^{*}$ (Figure 2.1), where there are no discrete eigenvalues [40]. This dividing value, $-\lambda^{*}$, is sometimes called the Corngold limit and marks the physically minimum possible $\alpha$ eigenvalue. For a one-speed infinite medium, Eq. (2.61) shows this relationship when $k_{\infty}$ and $\Sigma_{s}$ are zero [33]. These unique behaviors are in part due to the slab geometry, in which it is possible for a particle traveling in a direction parallel to one of the faces to stream for an arbitrarily long time before suffering a collision or leaving the slab [40]. This complicates extending some of these observations to more complex media.

Further studies show that for finite media where the minimum velocity $v_{\min }>0$, the continuum disappears and the spectrum of $\mathbf{A}$ contains only the point spectrum, the $\alpha$ eigenvalues [38]. Point and line spectra fill the half-space of the continuum (Figure 2.1). The study asserts that the spectrum of $\mathbf{A}$ contains the continuum only if neutrons are allowed to exist at arbitrarily small speeds, $v_{\min }=0$. The lines are traces resulting from considering multiple neutron speeds: $\alpha$ eigenvalues depend on the speed, and as the speed varies from $v$ to $v_{\min }$, some eigenvalues remain stationary and others trace out curves or loops in the complex plane [4].

There is some experimental support that the $v_{\min }>0$ case is a more accurate representation of physical reality. This is expected, as the transport equation treats neutrons as classical particles and does not account for the quantum mechanical effects that a neutron encounters when its wavelength is comparable to a mean free path. The transport equation is invalid for such low energies, and another equation is necessary to study the effect on the spectrum [4]. In a Monte Carlo random walk, an energy cutoff is set such that when neutrons reach very low energies, the history is terminated. This is in part due to the absence of cross section data at very low neutron energies. As the TRMM uses Monte Carlo methods, calculated results consist only of the point and line spectra. 


\section{Delayed and Prompt Eigenvalues and Eigenfunctions}

The $\alpha$ eigenvalues fall into two categories: delayed and prompt [41]. Delayed $\alpha$ eigenvalues are close to the decay constants of the neutron precursor groups $\lambda_{j}, j=1$, $2, \cdots, J$. Prompt $\alpha$ eigenvalues are much greater in magnitude than the decay constants $\left|\alpha_{i}\right| \gg \lambda_{j}$. This difference is due to the slow precursor decay rate relative to the rate at which neutrons interact: prompt effects decay much faster than the decay of delayed neutron precursors.

The largest prompt eigenvalue, i.e., the largest $\alpha$ eigenvalue that satisfies Eq. (2.54), is known as the prompt fundamental eigenvalue $\alpha_{p 0}$. In many applications for subcritical problems, this is the eigenvalue of interest because of its use in calculating the subcriticality of the problem. In the subcritical case, a delayed eigenvalue serves as the true fundamental eigenvalue because the prompt fundamental eigenvalue is more negative. For supercritical problems, the prompt fundamental eigenvalue is always the true fundamental eigenvalue.

Accounting for delayed neutron precursors limits the fundamental eigenvalue in subcritical systems. Combining Eqs. (2.49) and (2.50) yields

$$
\frac{\alpha}{v} \phi+\mathbf{M} \phi(\mathbf{r}, E)=\mathbf{F}_{\mathbf{p}} \phi+\sum_{j=1}^{J} \frac{\lambda_{j}}{\alpha+\lambda_{j}} \chi_{j} \mathbf{F}_{\mathbf{d} j} \phi,
$$

introducing $J$ discontinuities at $\alpha=-\lambda_{j}$. When $\alpha \rightarrow-\lambda_{j}$, the last term in Eq. (2.82) either increases considerably if $\alpha>-\lambda_{j}$ (approaching from $-\lambda_{j}^{+}$) or decreases considerably if $\alpha<-\lambda_{j}$ (approaching from $-\lambda_{j}^{-}$). This introduces $J$ more $\alpha$ 's that balance Eq. (2.82): the delayed eigenvalues $\alpha_{d}$. If the system is very subcritical, $\alpha_{d j} \approx \lambda_{j}$, and the precursor decay constant that is smallest in magnitude is the fundamental eigenvalue: it is useless in determining the reactivity of the system. This is the condition shortly after a reactor is shut down, where the delayed neutron precursors present in the fuel continue to decay and emit delayed neutrons according to the form dictated by the delayed $\alpha$ eigenpairs.

Relative to the prompt eigenfunctions $\phi_{p}$, the $J$ delayed eigenfunctions $\phi_{d}$ are simpler. Delayed eigenvalues are all-real, as are the eigenfunctions. Spatially, the delayed eigenfunctions are similar to one another because the precursor concentrations depend on the fuel location. This is less true for the energy phase space, as each precursor emits neutrons with a different characteristic energy spectrum. For very subcritical systems with stationary fuel, the delayed eigenfunctions are very close to the prompt fundamental eigenfunction, and for some analysis methods they are 
assumed to be the same [41]. If the fuel is mobile, then the spatial variation of the delayed eigenfunctions changes because precursors migrate with the fuel.

With delayed neutron precursors, there are two additional classifications for the critical case in Eq. (2.57) for slightly supercritical systems where $k \cong 1^{+}$. Define the static reactivity insertion as [2]

$$
\rho_{0}=\frac{k-1}{k} .
$$

With reactivity insertions in the range $0<\rho_{0}<\beta$, the system is in a delayed or subprompt critical condition. This is because the delayed neutron precursor source is making the system critical: in their absence, the system would be subcritical. The fundamental eigenvalue $\alpha_{0}$ is positive, but on the order of the delayed neutron precursor decay constants, so the increase of the neutron population occurs relatively slowly. This provides time for implementing control measures in a delayed critical reactor as discussed in $\S 2.1$. With reactivity insertions in the range $\rho_{0}>\beta$, the system is in a prompt or superprompt critical condition [3]. Here, the system is supercritical due to prompt neutron effects alone. The fundamental eigenvalue $\alpha_{0}$ is a positive prompt eigenvalue much larger than a delayed eigenvalue: the neutron population increases very quickly. Without delayed neutron precursors, reactors would have to operate near this precarious state: the value of $\beta$ acts as forgiving reactivity buffer.

\subsubsection{Eigenfunction Expansion}

The functional time expansion approximation [9] of the neutron flux and precursor concentration in matrix form is

$$
\left[\begin{array}{c}
\phi(\mathbf{r}, E, t) \\
\boldsymbol{C}(\mathbf{r}, t)
\end{array}\right] \cong \sum_{i=0}^{m} T_{i}(t)\left[\begin{array}{c}
\phi_{i}(\mathbf{r}, E) \\
\boldsymbol{C}_{i}(\mathbf{r})
\end{array}\right],
$$

where $C$ is a length $J$ vector of the precursor group concentrations, $m$ is the total number of eigenvalues, and the time function $T_{i}(t)$ is left undefined. The spatial functions, $\left[\phi_{i}(\mathbf{r}, E) \boldsymbol{C}_{i}(\mathbf{r})\right]$, depend on the system configuration, while the time function also depends on source characteristics. Substituting this expansion into Eqs. (2.10) and (2.11) yields the matrix problem in terms of the new expanded flux and precursor 
concentration

$$
\begin{aligned}
\sum_{i=0}^{m} \frac{d T_{i}(t)}{d t} & {\left[\begin{array}{cc}
v^{-1} & 0 \\
0 & I
\end{array}\right]\left[\begin{array}{c}
\phi_{i}(\mathbf{r}, E) \\
\boldsymbol{C}_{i}(\mathbf{r})
\end{array}\right] } \\
& =\sum_{i=0}^{m} T_{i}(t)\left[\begin{array}{cc}
-\mathbf{M}+\mathbf{F}_{\mathbf{p}} & \boldsymbol{\chi} \boldsymbol{\lambda} \\
\mathbf{F}_{\mathbf{d}} & -\boldsymbol{\lambda}
\end{array}\right]\left[\begin{array}{c}
\phi_{i}(\mathbf{r}, E) \\
\boldsymbol{C}_{i}(\mathbf{r})
\end{array}\right]+S(\mathbf{r}, E, t) .
\end{aligned}
$$

Multiplying the first term on the right side of the equation by the speed matrix and its inverse yields the forward $\alpha$-eigenvalue problem defined in Eq. (2.52). Replacing the matrix problem with the $\alpha$ eigenvalue, multiplying by the adjoint eigenfunction $\left[\phi_{n}^{\dagger}(\mathbf{r}, E) \boldsymbol{C}_{n}^{\dagger}(\mathbf{r})\right]$, and integrating over space and energy yields

$$
\sum_{i=0}^{m} \gamma_{n i} \frac{d T_{i}(t)}{d t}=\sum_{i=0}^{m} \alpha_{i} \gamma_{n i} T_{i}(t)+\left\langle\phi_{n}^{\dagger}, S(\mathbf{r}, E, t)\right\rangle_{\mathbf{r}, E},
$$

where

$$
\gamma_{n i}=\left\langle\phi_{n}^{\dagger}, v^{-1} \phi_{i}\right\rangle_{\mathbf{r}, E}+\left\langle\boldsymbol{C}_{n}^{\dagger}, \boldsymbol{C}_{i}\right\rangle_{\mathbf{r}},
$$

and the integral notation is

$$
\left\langle f^{\dagger}(\mathbf{r}, E), f(\mathbf{r}, E)\right\rangle_{\mathbf{r}, E}=\int_{V} \int_{0}^{\infty} f^{\dagger}(\mathbf{r}, E) f(\mathbf{r}, E) d E d V .
$$

The quantity $\gamma_{n i}$ is zero unless $n=i$ : this is known as the bi-orthogonality condition of the forward and adjoint functions,

$$
\gamma_{n i}=\gamma_{i i} \delta_{n i}=\gamma_{i} \delta_{n i} .
$$

Applying this condition yields $i$ first-order differential equations describing the behavior of the time expansion functions, $T_{i}(t)$, each satisfying the equation

$$
\frac{d T_{i}(t)}{d t}=\alpha_{i} T_{i}(t)+\frac{\left\langle\phi_{i}^{\dagger}, S(\mathbf{r}, E, t)\right\rangle_{\mathbf{r}, E}}{\gamma_{i}},
$$

where the external source $S(\mathbf{r}, E, t)$ is arbitrarily chosen after computation of the $\alpha$ eigenpairs. 


\section{Time Function Solutions}

A common assumption is that there is no external source after $t=0$, such as a short pulse, or some given flux distribution at time $t=0^{-}$. For both of these cases, $S(\mathbf{r}, E, t)=0$ for the times of interest, $t>0$, and Eq. (2.90) has the exponential time-dependent solution

$$
T_{i}(t)=A_{i} \exp \left(\alpha_{i} t\right),
$$

where the coefficients, $A_{i}$, depend on the source characteristics. In the case of a pulsed system, the source is mathematically described as a delta function in time, $S_{0}(\mathbf{r}, E) \delta(t)$. Inserting this into Eq. (2.90) and operating by $\int_{-\epsilon}^{\epsilon}(\cdot) d t$ yields the coefficients

$$
A_{i}=\frac{\left\langle\phi_{i}^{\dagger}, S_{0}(\mathbf{r}, E)\right\rangle_{\mathbf{r}, E}}{\gamma_{i}},
$$

for the eigenfunction expansion of a system reacting to a single pulse.

After several pulses, delayed precursors build up in the fuel: there are two ways of accounting for these concentrations. Superposition of the single pulse solution with a time delay yields an accurate result as long as the delayed neutron data is sufficiently accurate. The alternative solution is to describe the delayed precursor concentrations and include them while calculating the expansion coefficients. Assume that the spacedependent delayed neutron precursor concentrations at time $t=0^{-}$is $S_{j}(\mathbf{r})$. With this initial condition, the coefficients become

$$
A_{i}=\frac{\left\langle\phi_{i}^{\dagger}, S_{0}(\mathbf{r}, E)\right\rangle_{\mathbf{r}, E}+\left\langle\boldsymbol{C}_{i}^{\dagger}, S_{j}(\mathbf{r})\right\rangle_{\mathbf{r}}}{\gamma_{i}} .
$$

But, knowing the delayed neutron precursor concentrations requires some sort of a priori approximation, so it is perhaps better to use superposition or both methods for verification.

A similar approach is used when considering some initial flux distribution, where $S_{0}(\mathbf{r}, E)$ in the previous equation is recast as the appropriate initial condition describing the flux at $t=0^{-}$. Such a problem is useful in describing cases where the flux response to a sudden change in materials, densities, or geometries is of interest. In this case, the $\alpha$ eigenpairs are those of the "changed" system, and the initial conditions for the flux and precursor concentrations are solutions to the steady-state problem considering some time of operation.

The time functions for systems with an external source for times $t>0$ are solutions 
to the first-order inhomogeneous equation

$$
\frac{d T_{i}(t)}{d t}-\alpha_{i} T_{i}(t)=\frac{\left\langle\phi_{i}^{\dagger}, S(\mathbf{r}, E, t)\right\rangle_{\mathbf{r}, E}}{\gamma_{i}}
$$

Multiplying this equation by the integrating factor $\exp \left(-\alpha_{i} t\right)$ and solving yields

$$
T_{i}(t)=\left[\frac{1}{\gamma_{i}} \int^{t}\left\langle\phi_{i}^{\dagger}, S\left(\mathbf{r}, E, t^{\prime}\right)\right\rangle_{\mathbf{r}, E} \exp \left(-\alpha_{i} t^{\prime}\right) d t^{\prime}+C\right] \exp \left(\alpha_{i} t\right),
$$

which is Eq. (2.91) with an extra integral term. The $S\left(\mathbf{r}, E, t^{\prime}\right)$ term in the integral has some arbitrary time dependence, the least complex of these being a constant source turned on at $t=0$, considering nothing beforehand, $T_{i}(0)=0$. In this case, the time function is

$$
T_{i}(t)=\frac{\left\langle\phi_{i}^{\dagger}, S(\mathbf{r}, E)\right\rangle_{\mathbf{r}, E}}{\alpha_{i} \gamma_{i}}\left[\exp \left(\alpha_{i} t\right)-1\right]
$$

More complex time-dependent sources are left from discussion.

In summary, the eigenfunction expansion of the time-dependent flux and precursor concentrations are

$$
\begin{gathered}
\phi(\mathbf{r}, E, t)=\sum_{i=0}^{M} A_{i} \phi_{i}(\mathbf{r}, E) \exp \left(\alpha_{i} t\right) \\
\boldsymbol{C}(\mathbf{r}, t)=\sum_{i=0}^{M} A_{i} \boldsymbol{C}_{i}(\mathbf{r}) \exp \left(\alpha_{i} t\right)
\end{gathered}
$$

for no source $t>0$ and

$$
\begin{aligned}
\phi(\mathbf{r}, E, t) & =\sum_{i=0}^{M} \alpha_{i}^{-1} A_{i} \phi_{i}(\mathbf{r}, E)\left[\exp \left(\alpha_{i} t\right)-1\right], \\
\boldsymbol{C}(\mathbf{r}, t) & =\sum_{i=0}^{M} \alpha_{i}^{-1} A_{i} \boldsymbol{C}_{i}(\mathbf{r})\left[\exp \left(\alpha_{i} t\right)-1\right]
\end{aligned}
$$

for a fixed constant source, where $M \leq m$ is the number of desired eigenfunctions in the expansion. As $M$ becomes large, the eigenfunction expansion accurately captures shorter and shorter time fluctuations. For many applications, only the time-dependent flux is of interest, and it is unnecessary to track the precursor concentrations after their use in determining the expansion coefficients. 
The $M$ must be chosen such that, if the expansion includes a complex eigenvalue, then it also includes its complex conjugate. Complex eigenvalues only have a physical meaning when used as a pair. Mathematically, without complex eigenvalues, the flux contribution of every higher mode only decreases. But in a physical system, neutrons slow down and induce fission, emitting neutrons at higher energies. Thus, the flux at a higher energies experiences a decrease as neutrons slow down and an increase as thermal neutrons fission. This oscillatory motion is described by complex $\alpha$ eigenvalues. Consider the sum of the two elements $i$ and $i^{\prime}$ of Eq. (2.97), where $\alpha_{i}$ and $\alpha_{i^{\prime}}$ are conjugate pairs

$$
\phi_{i}(\mathbf{r}, E, t)+\phi_{i^{\prime}}(\mathbf{r}, E, t)=A_{i} \phi_{i}(\mathbf{r}, E) \exp \left(\alpha_{i} t\right)+A_{i^{\prime}} \phi_{i^{\prime}}(\mathbf{r}, E) \exp \left(\alpha_{i^{\prime}} t\right) .
$$

The flux solution takes the form

$$
\begin{aligned}
& \phi_{i}(\mathbf{r}, E, t)+\phi_{i^{\prime}}(\mathbf{r}, E, t) \\
& \quad=\left[A_{i} \phi_{i}(\mathbf{r}, E) \exp \left(\operatorname{Im}\left(\alpha_{i}\right) t\right)+A_{i^{\prime}} \phi_{i^{\prime}}(\mathbf{r}, E) \exp \left(\operatorname{Im}\left(\alpha_{i^{\prime}}\right) t\right)\right] \exp \left(\operatorname{Re}\left(\alpha_{i}\right) t\right) .
\end{aligned}
$$

The combination of the two terms in the brackets describes an oscillation dictated by the imaginary part of the eigenvalues. An overall exponential decay dictated by the real part of the eigenvalues damps the oscillation, where $\operatorname{Re}\left(\alpha_{i}\right)=\operatorname{Re}\left(\alpha_{i^{\prime}}\right)<0$. Both the coefficients and eigenfunctions have imaginary parts that cancel out inside the parenthesis.

Only the coefficients depend on source characteristics: the $\alpha$-eigenpair calculations need no information about the initial source. This allows for generating timedependent solutions for any arbitrary initial source for the same system. Direct solution methods define the source before calculating solutions: using a different source requires running the analysis again with the updated source characteristics, which is costly computationally, but does allow for accommodating of temperature feedback.

The coefficients determine the contribution of each eigenfunction to the total solution. For example, in the case that the fundamental eigenfunction is all-negative, the coefficient corresponding to that eigenfunction is also negative. In the case that the solution behaves symmetrically, the coefficients for asymmetric eigenfunctions are very small relative to coefficients of symmetrical eigenfunctions. Together, the coefficients and eigenfunctions are known as the kinetic modes, $A_{i} \phi_{i}(\mathbf{r}, E)$, of the problem. With the solutions to the forward and adjoint $\alpha$-eigenvalue problems, this formulation presents a complete approach to approximate the time-dependent neutron flux and precursor concentrations. The importance equation and the adjoint $\alpha$-eigenvalue 
problem are discussed in $\S 3.1 .2$.

\section{Completeness}

The functional time expansion in Eq. (2.84) is an approximation dependent on the size of $m$. It was initially thought that $\mathbf{A}$ possesses a complete set of eigenfunctions, i.e., $m \rightarrow \infty$, in which case the expansion is equivalent to the time-dependent solution [40]. But, it has been shown that one-dimensional media have a finite number of eigenvalues, in which case the flux expansion is better described as

$$
\phi(\mathbf{r}, E, t)=\sum_{i=0}^{m} T_{i}(t) \phi_{i}(\mathbf{r}, E)+\zeta(\mathbf{r}, E, t),
$$

where the residual term $\zeta(\mathbf{r}, E, t)$ is an inseparable part of the flux solution and is small compared to the other expansion terms. The residual term goes to zero as $t \rightarrow \infty$ : despite incompleteness, it is possible to obtain an approximate eigenfunction expansion with a small error term [42]. For example, consider a small slab with only $m=2$ eigenvalues. Without the residual term, the description of the solution with only two eigenfunctions is unable to describe sharp flux shapes in the case of an asymmetric plane source for the earliest times.

This assertion of incompleteness hinges on two factors: the slab geometry and the existence of the continuum without point spectrum. Physical situations unique to planar geometry are noted in $\S 2.3 .3$. The existence of the continuum limits the $\alpha$ eigenvalues to the half-space to the right of $-\lambda^{*}$ (Figure 2.1). But, in cases with more physical geometries where the minimum neutron speed is positive, there is no continuum, and $\alpha$ eigenvalues fill the half-space to the left of $-\lambda^{*}$ : this makes the expansion more complete and decreases the residual term. While the completeness of the eigenfunctions has not been rigorously proven, this expansion has empirically shown to be accurate for more realistic problems: it is at least safe to assume that $m$ is large for physical problems. This reflects the case for computational methods, as the number of eigenfunctions calculated are ideally large, but always finite. Thus, there will always be some residual term representing an inseparable part of the flux solution.

\subsection{Measuring $\alpha$ Eigenvalues}

Pulsed-neutron and Rossi- $\alpha$ experiments measure the prompt fundamental neutron decay constant, the eigenvalue $\alpha_{p 0}$, for its use in determining the subcriticality or 
kinetic parameters of a system. It is sometimes possible to measure one or two higher prompt eigenvalues, but the validity and accuracy of these higher eigenvalues is questionable: determination of the eigenvalues are based on only a few measurements due to the short decay time of higher kinetic modes and the interference of the prompt fundamental mode. Measuring delayed eigenvalues is easier due to their

long decay time, but these are generally of much less interest either because in they are unimportant or their behavior is already known.

Experimentally, the eigenfunctions always appear in the form of the kinetic modes $A_{i} \phi_{i}(\mathbf{r}, E)$. While it is possible to spatially measure the entire prompt fundamental kinetic mode $A_{p 0} \phi_{p 0}(\mathbf{r})$, it requires an unreasonable number of detectors. Measuring any higher kinetic mode is incredibly difficult. Thus, measurements from a few detectors determine the spatial variation of the flux isolated to a few locations, and inferences about the prompt and delayed kinetic modes are made from these.

\subsubsection{Pulsed-Neutron Experiments}

The procedure of a PNE is to instantaneously insert a large number of neutrons into a subcritical system and measure the flux time response following this pulse with detectors. From the time response, the reactivity of the system is inferred using one of several methods, two of which are the inhour and area-ratio method. Near critical, both methods are of comparable accuracy, but further from critical, the inhour method is less sensitive to calculation errors and is more accurate [41].

An ideal detector response (Figure 2.2) is characterized by three distinct time intervals: (I) a short interval following the pulse in which fast source neutrons slow down and higher prompt modes decay, (II) an intermediate interval in which the detector response decays exponentially according to the fundamental decay constant, and (III) a long interval in which delayed modes dominate the detector response. Because delayed modes decay slowly, the behavior of interval III is dependent on the number of previous pulses. With one pulse, interval III will be a result of delayed fissions propagating from that single pulse. With many pulses, each successive pulse contributes some delayed neutrons to interval III, albeit in lesser and lesser amounts as time progresses. The system will reach "constant delayed neutron background" with enough pulses, characterized by the detector response being nearly identical from pulse to pulse. Because of the short time scale of a quarter second (Figure 2.2), the delayed neutron contributions look constant: they are in fact decaying very slowly.

With the fundamental prompt mode decay constant taken from interval II of the 


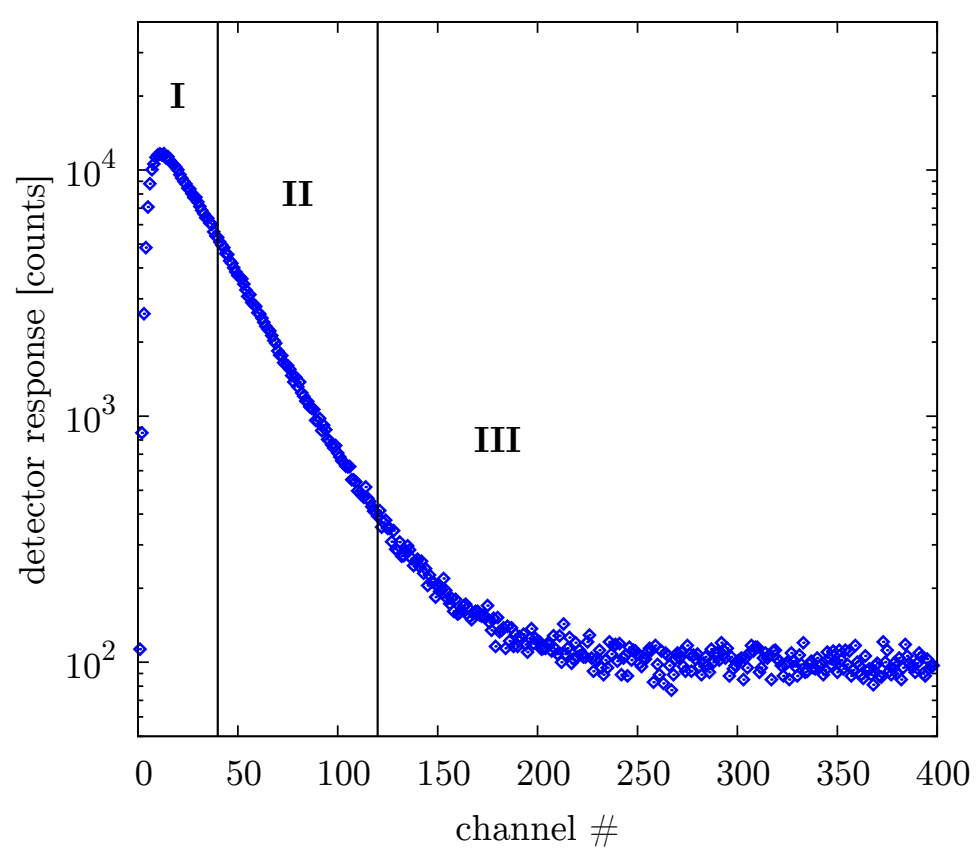

Figure 2.2: The measured detector response to a neutron pulse plotted on the appropriate time scale shows the three characteristic time intervals dominated by the different kinetic modes.

measured detector response, the reactivity is inferred using the inhour equation

$$
\rho=\alpha_{p 0} \Lambda+\bar{\beta}
$$

given the effective delayed neutron fraction $\bar{\beta}$ and a calculated neutron generation time $\Lambda$. The accuracy of this method depends on finding a proper exponential fit for the measured detector response in interval II and the calculation of a proper neutron generation time, which varies with system parameters. For large reactors with little leakage, the effective delayed neutron fraction is nearly the same as the total delayed neutron fraction.

An alternative way of calculating the reactivity of the system uses integrals of the detector responses, which is the area under the curves. This method depends on the system reaching constant delayed neutron background to provide the best statistics and an assumption in the derivation of the reactivity-area relation. Derivation of this method starts from point kinetics with Eqs. (2.31) and (2.32). Describe the source as a delta function at $t=0$ with strength $S_{0}$. Rename the amplitude function to $P(t)$ and split it into prompt and delayed contributions to the time response. For the prompt amplitude, ignore the delayed neutron precursors because the prompt modes 
decay very quickly. The point kinetics equations for the prompt contribution simplify to

$$
\frac{d P_{p}(t)}{d t}=\frac{\rho-\beta}{\Lambda} P_{p}(t)+S_{0} \delta(t)
$$

Because the prompt contribution is identical for each pulse, the initial condition is $P_{p}(0)=P_{p}(T)$. Integrating this equation over the time between pulses, $0<t<T$, and rearranging the result yields the prompt area

$$
A_{p}=\int_{0}^{T} P_{p}(t) d t=\frac{S_{0} \Lambda}{\beta-\rho} .
$$

Obtain the total neutron area in a similar manner, except include the delayed neutron precursors in the formulation. Provided that the delayed neutron background is constant, each pulse is identical and the initial conditions $T_{t}(0)=T_{t}(T)$ and $c(0)=$ $c(T)$ hold. Integrate Eqs. (2.31) and (2.32) over the time between pulses and combine the resulting equations to eliminate the precursors. Rearranging yields the total area

$$
A_{t}=\int_{0}^{T} P_{t}(t) d t=-\frac{S_{0} \Lambda}{\rho}
$$

The ratio of the prompt to delayed neutron area yields the reactivity of the system in dollars

$$
\frac{A_{p}}{A_{t}-A_{p}}=-\frac{\rho}{\beta}
$$

Unfortunately, point kinetics is only approximate for a subcritical reactor because the shape function never follows the exact shape of the fundamental mode. Furthermore, the area-ratio method is spatially dependent because the detector is measuring the local variation of the neutron flux. A number of adjusted area-ratio methods have been developed to account for these spatial effects, such as the extrapolated area-ratio method and those with spatial correction factors [43]. The former method uses fitted equations to extrapolate the neutron flux back to $t=0$ in calculating the prompt and delayed neutron areas, while the spatial correction factors $F(\mathbf{r})$ adjust for variations brought about by the local measurement of the neutron flux [44]. Applying the correction to the area ratios yields

$$
\frac{\rho}{\beta}=-\frac{A_{p}}{A_{t}-A_{p}} F(\mathbf{r}) .
$$


For example, the correction factor

$$
F(\mathbf{r})=\frac{\phi_{d 0}(\mathbf{r}, E)}{\phi_{p 0}(\mathbf{r}, E)}
$$

corrects for kinetic distortion: the spatial-dependent difference between the prompt and delayed kinetic modes. This is an approximation considering higher kinetic modes as negligible.

\subsubsection{Rossi- $\alpha$ Experiments}

The Rossi- $\alpha$ formula gives the expected number of neutrons counted in an interval $d t$ at time $t$ after a neutron count at $t=0$ :

$$
R(t) d t=C d t+A \exp (\alpha t) d t
$$

where $C$ is the uncorrelated counting rate and $A \exp (\alpha t)$ represents the exponential time-dependence of the prompt fission chain caused by the original neutron [45]. The value describing this time-dependence is the prompt neutron decay constant, the eigenvalue $\alpha_{p 0}$, and $A$ is the correlation amplitude.

The uncorrelated counting rate is given by

$$
C=\frac{\epsilon S}{\nu_{c r}-\bar{\nu}}
$$

where $\epsilon$ is the efficiency of the detector in counts per fission, $S$ is the source strength in neutrons per second, and $\nu_{c r}$ is a measure of criticality defined as the adjusted value of $\bar{\nu}$ at which the assembly is critical. The correlated amplitude is given by

$$
A=\frac{\epsilon X_{2}}{2 \bar{\nu}^{2} \tau\left(1-k_{p}\right)},
$$

where $X_{2}$ is a measure of the variation of $\bar{\nu}$ from one fission to another, $\tau$ is the mean neutron lifetime, and $k_{p}$ is the prompt multiplication factor [45]. While the correlated part of the Rossi- $\alpha$ formula is a sum of all kinetic modes, most measurements are unable to observe the contributions of these modes. Thus, calculations neglect these higher modes and use the fundamental mode approximation, where the prompt fundamental decay constant is

$$
\alpha=\alpha_{p 0}=\frac{1-k_{p}}{\tau}
$$


Detailed derivations of these equations are found in the references [46].

Necessary equipment for a Rossi- $\alpha$ experiment include an assembly, detector, and a source. The procedures include a few steps: (i) measure the counting rate $R(t)$ following the detection of one neutron at time $t=0$, (ii) build the curve and subtract the uncorrelated contribution, and (iii) perform a linear regression of the logarithm of the correlated contribution to obtain the prompt fundamental eigenvalue and the correlation amplitude [46]. Rossi- $\alpha$ experiments are suited for fast, small assemblies where the delayed neutrons are unimportant, like fast-burst reactors. In these reactors, the higher prompt kinetic modes decay very quickly and have little effect on the measurement. These experiments are useful in calculating kinetics parameters, such as the prompt neutron generation time or the effective delayed neutron fraction [47].

\subsection{Calculating $\alpha$ Eigenvalues}

Numerical methods developed for calculating $\alpha$ eigenvalues fall under a broad range of methodologies due to varying intents and purposes. Some methods calculate only the prompt fundamental eigenvalue $\alpha_{p 0}$, while others calculate delayed or higher prompt eigenvalues or the fundamental eigenfunction as well. Others calculate a number of higher prompt eigenpairs for modal analyses or calculation of correction factors. In any case, the calculated spectra of $\alpha$ eigenvalues are always finite. It is important to discuss these existing methods to find where the TRMM fits amongst them.

The list of methods detailed in this section are not all-inclusive, but are representative of the research performed on $\alpha$-eigenvalue calculations over the years. This section categorizes methods into four groups: fixed-source calculations, diffusion theory methods, transport theory methods, and Monte Carlo methods. Each review is clearly marked for easy reference, and a summary follows the individual discussions.

\subsubsection{Fixed-Source Calculations}

While these methods are able to appropriately account for temperature feedback, variations on time-dependent fixed-source calculations represent the most straightforward and least elegant way of obtaining the $\alpha$ eigenvalue of a system. In these calculations, deterministic or Monte Carlo methods provide the time-dependent flux shape, or flux at a point, of a system reacting to some initial external source. Exponential fits on the time-dependent fluxes yield the prompt fundamental eigenvalue $\alpha_{p 0}$ of the system. This is equivalent to the direct simulation of a PNE within a code; therefore, these methods have all the pitfalls of a PNE, including difficulty finding 
higher eigenvalues and determination of the kinetic modes.

Most of the literature on these calculations use Monte Carlo codes to determine the time-dependence of the flux. These include standard production codes able to handle time dependence [48] and codes specifically written to handle time-dependent problems [49]. In the former study, the continuous time space is split into channels of width $\Delta t$ and the code tracks the time-dependent flux among those channels. In the latter study, the code generates the time-dependent flux via a censusing technique. For both studies, the calculated prompt fundamental eigenvalue compares well to measured data, but neither yields higher eigenpairs. The following example problem uses the standard production code MCNP5 [34] to calculate the flux time-dependence in the FSV reactor to obtain the prompt fundamental eigenvalue and reactivity of the configuration.

Method 1: With proper tallies and source definitions, MCNP5 accurately simulates the time-dependent flux in a PNE. A detailed FSV MCNP5 model with homogenized fuel rods [50] is subject to a pulsed source. Due to the computational cost of modeling several hundred pulses, MCNP5 simulates the response to a single pulse, and superposition yields the constant delayed neutron background. The neutron flux tally divides the flux into time bins to simulate the multichannel analyzer used in the experiment, minus the dead time losses. This flux tally is weighted by the ${ }^{10} \mathrm{~B}$ absorption cross section to simulate the boron-lined detector response, without efficiency losses. MCNP5 tallies this weighted flux in three regions: 16, 30, and 31. Additional variance reduction techniques increase flux tally statistics: increasing the number of delayed neutrons released per fission and geometry splitting with Russian roulette.

A least-squares analysis [51] of the calculated detector response data yields a fit to the specific form dictated by the series of exponentials in the eigenfunction expansion. The inhour method yields the reactivity using the prompt fundamental eigenvalue of the fit. For the area-ratio method, the integrals of the fitted solutions yield the prompt and delayed areas.

MCNP5 calculates detector responses without and with delayed neutron emission (Figures 2.3 and 2.4). Without delayed neutrons, the emission probability is set to zero, yielding only the prompt neutron contribution to the detector response. By channel 200, the response is very noisy due to the fundamental prompt mode decreasing several orders of magnitude. With delayed neutrons, counts after channel 200 increase considerably, but still have a low yield: one benefit of having constant delayed neutron background is an increased number of counts in interval III. Detector 


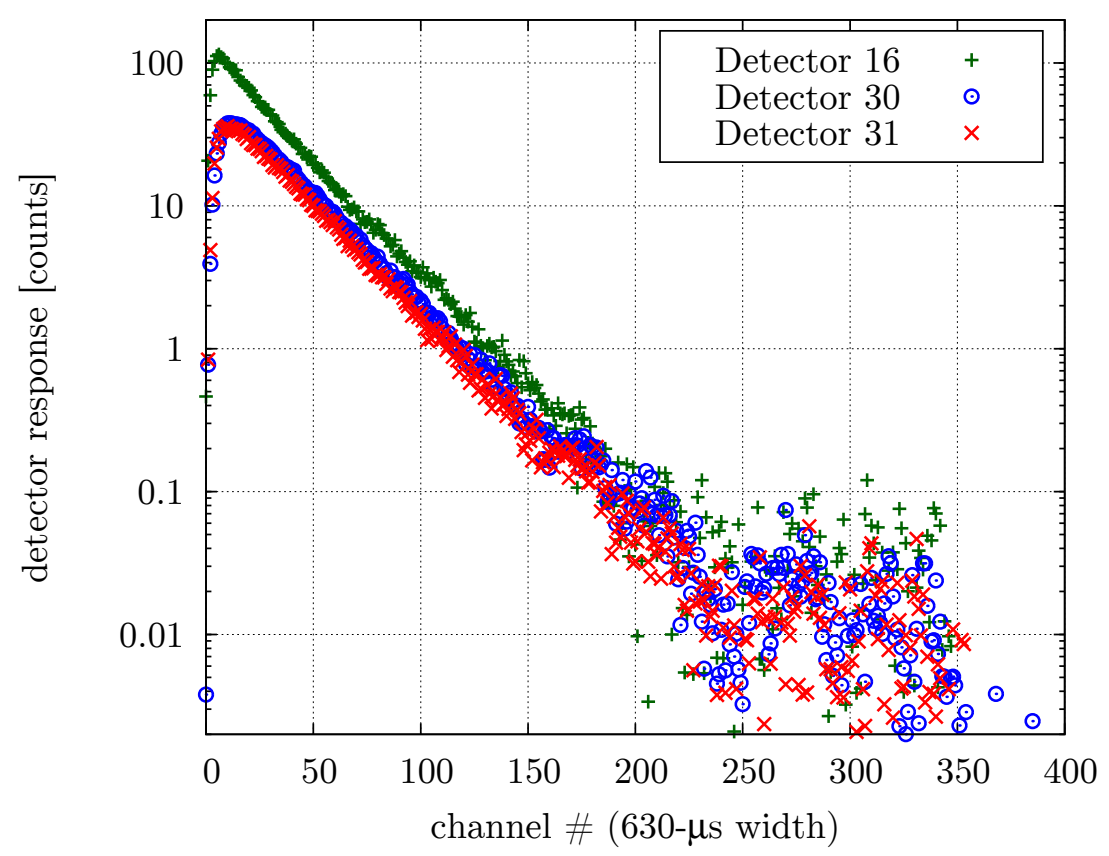

Figure 2.3: MCNP5-calculated prompt detector responses for a pulsed subcritical configuration of FSV yields very few counts in interval III due to the exponentially decaying prompt fundamental mode.

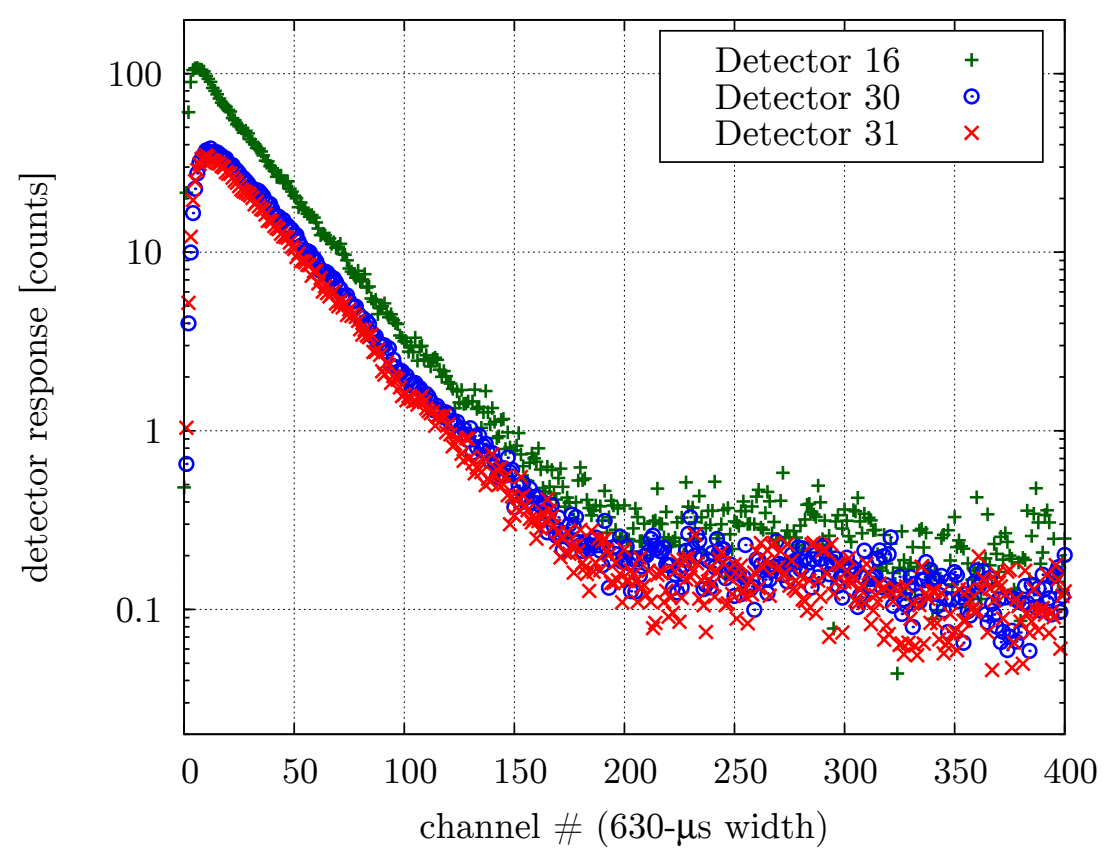

Figure 2.4: MCNP5-calculated detector responses with delayed neutron precursors shows an increased number of counts in interval III due to the contribution of delayed neutron emission. 
16 has the largest in amplitude response because it is closest to the source: detectors 30 and 31 are in adjacent regions nearly equidistant from the source.

To provide a fair comparison, the same least-squares analysis and reactivity calculations performed on the calculated detector responses are repeated on the measured results. Decay constants and areas are averaged values from the three detectors (Table 2.3). This particular configuration is relatively further from critical, so the inhour method is expected to return more accurate results than the area-ratio method.

The inhour method yields the best $k_{\text {eff }}$ for both measured and MCNP5 results, while the extrapolated area-ratio method produces more inaccurate results stemming from a pair of issues: inadequate spatial correction factors and poor statistics in the delayed neutron region. Monte Carlo simulations calculating the detector responses (Figure 2.4) already use long runtimes and several variance reduction techniques, so a better representation of delayed neutron emission is necessary to yield more counts in interval III. Still, the fitted fundamental decay constant matches very closely to the measured constant.

Table 2.3: Comparisons of measured and MCNP5-calculated results for one subcritical configuration of the FSV PNE.

\begin{tabular}{cccccc}
\hline Data & \multicolumn{2}{c}{ Area-ratio } & \multicolumn{2}{c}{ Inhour } & Calculated \\
source & $\rho / \beta$ & $k_{\text {eff }}$ & $\alpha$ & $k_{\text {eff }}$ & $k_{\text {eff }}$ \\
\hline Measured & -2.490 & 0.984 & -57.522 & 0.9826 & 0.981 \\
MCNP5 & -3.206 & 0.980 & -56.396 & 0.9830 & 0.9825 \\
\hline
\end{tabular}

\subsubsection{Diffusion Theory Methods}

The following methods use the multigroup diffusion equations. Different procedures for calculating eigenpairs use iterative, elimination, or matrix methods.

Method 2: One study demonstrates the ability to calculate the delayed fundamental eigenvalue $\alpha_{d 0}$ of a subcritical system in the context of a standard criticality code [52]. This is an iterative method:

- Start with an estimate of the delayed eigenfunction $\phi_{d 0}^{(n)}$ and delayed eigenvalue $\alpha_{d 0}^{(n)}$.

- Apply this eigenvalue estimate to Eq. (2.82): add the time-absorption term $\alpha / v$ to the absorption cross section and add the eigenvalue to the denominator of the delayed fission emission spectrum.

- Run a criticality, or $k$-eigenvalue, calculation to update the delayed eigenfunction $\phi_{d 0}^{(n+1)}$. 
- The largest root of the equation

$$
\rho^{*}=\alpha \Lambda^{*}+\alpha \sum_{j} \frac{\beta_{j}}{\alpha+\lambda_{j}},
$$

is the new delayed fundamental eigenvalue $\alpha_{d 0}^{(n+1)}$, where

$$
\begin{aligned}
\rho^{*} & =1-\frac{\iint \Sigma_{a} \phi_{d}\left(\mathbf{r}, E^{\prime}\right) d E^{\prime} d V+\iint n \cdot J\left(\mathbf{r}, E^{\prime}\right) d E^{\prime} d S}{\iint \bar{\nu} \Sigma_{f}\left(\mathbf{r}, E^{\prime}\right) \phi_{d}\left(\mathbf{r}, E^{\prime}\right) d E^{\prime} d V}, \\
\Lambda^{*} & =\frac{\iint \frac{1}{v} \phi_{d}\left(\mathbf{r}, E^{\prime}\right) d E^{\prime} d V}{\iint \bar{\nu} \Sigma_{f}\left(\mathbf{r}, E^{\prime}\right) \phi_{d}\left(\mathbf{r}, E^{\prime}\right) d E^{\prime} d V},
\end{aligned}
$$

represent quantities similar to the reactivity and neutron generation time as given in Eqs. (2.22) and (2.26), respectively.

- Apply the new eigenvalue and repeat the criticality calculation until convergence, $k=1$.

This is known as a $k$ - $\alpha$ iteration. Numerical results show good agreement to analytical solutions for multigroup slab problems. This iterative method applies equally to the multigroup transport equation. Only the delayed eigenvalue is calculated.

Method 3: Another study applies a $k-\alpha$ iteration to calculate the prompt fundamental and all delayed eigenpairs. It uses an additional elimination method to calculate higher eigenpairs [53]. The iterative method starts calculating the fundamental eigenvalues, $i=0$ :

- Make the initial guess for $\alpha^{(n)}$ using a root of the inhour equation

$$
\rho_{i}=\alpha \Lambda_{0}+\alpha \sum_{j}^{J} \frac{\beta_{j}}{\alpha+\lambda_{j}},
$$

where $J$ is the total number of delayed neutron precursor groups. The reactivity $\rho_{i}$ and neutron generation time $\Lambda_{0}$ are calculated with the $i$ th and fundamental solutions of the $k$-eigenvalue problem, respectively. There are $J+1$ roots to this equation: $J$ roots near the delayed constants $\lambda_{j}$ and one much larger in magnitude. These roots are near the delayed and prompt $\alpha$ eigenvalues, respectively: the starting guess determines the calculated eigenvalue.

- Apply this eigenvalue to Eq. (2.82): add the time-absorption term to the absorption cross section and add the eigenvalue to the denominator of the delayed fission emission spectrum.

- If $i=0$, run a standard criticality calculation to update the eigenfunction $\phi^{(n+1)}$. 
- If $i>0$, run a criticality calculation with the elimination method to update the eigenfunction $\phi^{(n+1)}$.

- Update the eigenvalue $\alpha^{(n+1)}$, increasing or decreasing it depending upon the value of $k$.

- Apply the eigenvalue and repeat the criticality calculation until convergence, $k=1$.

- Repeat the process for each root of the inhour equation.

- Recalculate the roots of the inhour equation for $i=i+1$ and repeat the process until obtaining the desired number of higher eigenvalues.

The elimination method provides a way to obtain the $i$ th solution using both the forward and adjoint $k$-eigenvalue problems

$$
\begin{aligned}
\mathbf{M} \phi_{i} & =\frac{1}{k_{i}} \mathbf{F} \phi_{i}, \\
\mathbf{M}^{\dagger} \phi_{i}^{\dagger} & =\frac{1}{k_{i}^{\dagger}} \mathbf{F}^{\dagger} \phi_{i}^{\dagger},
\end{aligned}
$$

by expanding the starting guesses in terms of the $i$ th solutions

$$
\begin{aligned}
& \phi=a_{0} \phi_{0}+a_{1} \phi_{1}+a_{2} \phi_{2}+\cdots, \\
& \phi^{\dagger}=b_{0} \phi_{0}^{\dagger}+b_{1} \phi_{1}^{\dagger}+b_{2} \phi_{2}^{\dagger}+\cdots,
\end{aligned}
$$

where the coefficients are

$$
\begin{array}{r}
a_{i}=\frac{\left\langle\phi_{i}^{\dagger}, \mathbf{F} \phi\right\rangle}{\left\langle\phi_{i}^{\dagger}, \mathbf{F} \phi_{i}\right\rangle}, \\
b_{i}=\frac{\left\langle\phi, \mathbf{F} \phi_{i}^{\dagger}\right\rangle}{\left\langle\phi_{i}, \mathbf{F} \phi_{i}^{\dagger}\right\rangle} .
\end{array}
$$

A criticality calculation with the elimination method starts with $i=1$, with the known fundamental solutions $\phi_{0}$ and $\phi_{0}^{\dagger}$ :

- Starting with the guess $\phi$, subtract the component along the fundamental solution $\phi-a_{0} \phi_{0}$. Perform the criticality calculation, periodically filtering out the fundamental solution to obtain $\phi_{1}$.

- Starting with the guess $\phi^{\dagger}$, subtract the component along the fundamental solution $\phi^{\dagger}-b_{0} \phi_{0}^{\dagger}$. Perform the adjoint criticality calculation, periodically filtering out the adjoint fundamental solution to obtain $\phi_{1}^{\dagger}$. 
- Starting with the guess $\phi$, subtract the components along the previously determined solutions $\phi-a_{0} \phi_{0}-a_{1} \phi_{1}$. Perform the criticality calculation, periodically filtering out the higher solutions to obtain $\phi_{2}$.

- Starting with the guess $\phi^{\dagger}$, subtract the components along the previously determined solutions $\phi^{\dagger}-b_{0} \phi_{0}^{\dagger}-b_{1} \phi_{1}^{\dagger}$. Perform the criticality calculation, periodically filtering out the higher solutions to obtain $\phi_{2}^{\dagger}$.

- Repeat the last two steps for each higher solution.

This elimination method applies to the standard criticality calculation, to obtain higher $k$ eigenpairs, and the $\alpha$-adjusted criticality calculation, to obtain higher $\alpha$ eigenpairs. Results from this method agree well with analytical slab solutions as well as two-dimensional few-group problems. For most problems, the higher delayed eigenpairs are unimportant because the fuel is stationary: they are only useful in a case where the fuel changes drastically.

Method 4: Another study formulates a matrix problem to compute the delayed and many prompt eigenpairs of the FSV reactor for PNE analysis. This represents an application of a method to an actual problem, with comparisons to measured results. The overall calculation procedure is extensive [54], so this section focuses on the modal method followed by the RZMODE and RTMODE codes for calculating the kinetic modes [55]. Of the diffusion methods, this is most similar to the TRMM due to the matrix formulation.

RZMODE and RTMODE are two-group diffusion codes with $r-z$ and $r-\theta$ geometry, respectively. They use the method of harmonics, expanding the group fluxes, $\phi^{1}$ and $\phi^{2}$, and precursor concentrations as the sums

$$
\begin{aligned}
\phi^{1}(\mathbf{r}, t) & =\sum_{\nu=1}^{N} W_{\nu}(\mathbf{r}) T_{\nu}^{1}(t), \\
\phi^{2}(\mathbf{r}, t) & =\sum_{\nu=1}^{N} W_{\nu}(\mathbf{r}) T_{\nu}^{2}(t), \\
C(\mathbf{r}, t) & =\sum_{\nu=1}^{N} W_{\nu}(\mathbf{r}) T_{\nu}^{c}(t),
\end{aligned}
$$

where the homogeneous reactor modes $W_{\nu}(\mathbf{r})$ are the solutions to the equation

$$
\nabla^{2} W_{\nu}(\mathbf{r})+B_{\nu}^{2} W_{\nu}(\mathbf{r})=0
$$


with the boundary condition

$$
W_{\nu}(\mathbf{r})=0,
$$

where $B_{\nu}$ is the geometric buckling. These homogeneous modes are combinations of sine or cosine and Bessel functions and are normalized such that

$$
\left\langle W_{\mu}(\mathbf{r}), W_{\nu}(\mathbf{r})\right\rangle=\delta_{\mu \nu} .
$$

Substituting these expansions into the two-group diffusion and precursor equations, multiplying by $W_{\mu}(\mathbf{r})$, and integrating analytically or numerically over the size $N$ spatial domain yields the full $3 N \times 3 N$ matrix problem. But, RZMODE and RTMODE only calculate the thermal and delayed kinetic modes because the fast kinetic modes decay very quickly: the full problem reduces to two $N \times N$ matrix problems for the prompt thermal and delayed kinetic modes with additional approximations. The last steps are to find the forward and adjoint eigenpairs of the two matrices, and calculate expansion coefficients for a given source. Two r- $\theta$ geometry calculations are necessary to account for the axial asymmetry in the FSV reactor.

There are several limitations of this method other than the diffusion approximation. The spatial representations are two-dimensional, it uses only two groups, and ignores fast modes. Additionally, it uses inadequate expansion modes: the homogeneous reactor modes are too simple to capture the thermal flux peaks in the reflector. Still, the calculated eigenvalues agree within $10 \%$ of measurements and the eigenfunctions are able to capture some of the flux variations [44].

\subsubsection{Transport Theory Methods}

The following methods use some form of the multigroup neutron transport equations to treat problems ranging from one-dimensional slabs and spheres to a complex ADS.

Method 5: One study uses an iterative method to find the eigenvalues of onespeed, one-dimensional slabs and spheres [56] by formulating a relation between the $\alpha$ - and $c$-eigenvalue transport problems

$$
\begin{aligned}
& \left(\alpha+\Sigma_{s}+\mu \frac{\partial}{\partial x}\right) \phi(x, \mu)=\frac{\Sigma_{s}}{2} \int_{-1}^{1} d \mu^{\prime} \phi\left(x, \mu^{\prime}\right) ; \quad x \in\left[-\frac{a}{\Sigma_{s}}, \frac{a}{\Sigma_{s}}\right], \\
& \left(\tilde{\Sigma}_{t}+\mu \frac{\partial}{\partial x}\right) \phi(x, \mu)=\frac{c \tilde{\Sigma}_{t}}{2} \int_{-1}^{1} d \mu^{\prime} \phi\left(x, \mu^{\prime}\right) ; \quad x \in\left[-\frac{\tilde{a}}{\tilde{\Sigma}_{t}}, \frac{\tilde{a}}{\tilde{\Sigma}_{t}}\right],
\end{aligned}
$$

where $a$ and $\tilde{a}$ are the the half-thicknesses or -radii of the $\alpha$ - and $c$-eigenvalue problems, respectively, and isotropic scattering is assumed. The neutron speed, omitted from 
the formulation, is set to unity $v=1$. These two eigenvalue problems are equal when $c$ and $\tilde{\Sigma}_{t}$ meet the conditions

$$
c=\frac{\Sigma_{s}}{\tilde{\Sigma}_{t}}=\frac{\Sigma_{s}}{\Sigma_{s}+\alpha}
$$

or, in terms of the problem geometry,

$$
c=\frac{a}{\tilde{a}}
$$

The method makes an additional assumption that $\tilde{\Sigma}_{t}=\Sigma_{s}=1$ : the units of the thicknesses are mean free paths. The iterative method repeats a $c$-eigenvalue calculation for different problem thicknesses $\tilde{a}$ :

- Choose the dimension of the $\alpha$-eigenvalue problem $a$ whose eigenvalues are desired.

- Guess an eigenvalue $\alpha$ close to the desired $i$ th eigenvalue.

- Calculate the initial critical problem dimension $\tilde{a}^{(0)}$ with Eqs. (2.133) and (2.134).

- Find $c_{i}^{(n)}$ numerically for a problem with this dimension $\tilde{a}^{(n)}$.

- Update the critical problem dimension $\tilde{a}^{(n+1)}$ using Eq. (2.134) or another method.

- Repeat the last two steps until convergence, $\left(\tilde{a} c_{i}\right)^{(N)}=a$.

- Transform the final value $c_{i}$ to $\alpha_{i}$ using Eq. (2.133).

Repeating the calculation with the next guess yields the higher $\alpha$ eigenvalues, until the entire physical search space above the continuum is interrogated. While the highly simplified medium facilitates the usage of this method, this method provides a benchmark used for verifications of other methods discussed here. It is able to obtain the majority of the eigenvalues for slabs and spheres of a range of sizes. Eigenvalues close to unity are difficult to obtain numerically because $c \rightarrow \infty$ for these cases.

Method 6: Another study uses Green's functions to model one-speed, multiplying, multi-region slabs and obtain boundary flux values for an eigenvalue search [33]. This is known as the Green's Function Method (GFM). The one-dimensional $\alpha$ eigenvalue equation with fission is

$$
\left[\frac{\alpha}{v}+\Sigma_{t}+\mu \frac{\partial}{\partial x}\right] \phi(x, \mu)=\frac{\Sigma_{s}}{2} \int_{-1}^{1} d \mu^{\prime} \phi\left(x, \mu^{\prime}\right)+\frac{\bar{\nu} \Sigma_{f}}{2} \int_{-1}^{1} d \mu^{\prime} \phi\left(x, \mu^{\prime}\right) .
$$

Application of the Green's function yields equations describing the interactions of the 
boundary angular fluxes. The GFM forms a matrix problem from these equations. It obtains the $\alpha$ eigenvalues from a search routine: it determines a search space and interrogates all values of $\alpha$ within that space. It only searches for eigenvalues above the continuum. After determining an $\alpha$ eigenvalue, the GFM calculates the boundary angular fluxes using an iterative process: this yields the eigenfunctions.

The GFM delivers benchmark-quality calculations for five multiple-part test problems, from purely-scattering, homogeneous slabs to multiplying, multi-region slabs. Results for eigenvalues and eigenfunctions for three problems match results obtained with other methods. Two additional problems provide results for multiplying slabs, including a loosely-coupled reactor problem.

Method 7: Another study forms a matrix problem from a discretized form of the one-speed, one-dimensional transport equation [57] using discrete ordinates for angular space and finite difference for position space. The $S_{n}$ method divides the angular variable $\mu$ into $M$ directions $\mu_{1}, \mu_{2}, \cdots, \mu_{M}$, each with weight $w_{m}$. Finite difference divides the spatial variable $x$ into $N$ meshes of uniform width $h$. Rearranging the $\alpha$-eigenvalue problem in Eq. (2.131) and introducing these discretizations yields $N M$ equations

$$
\mu_{m} \frac{\phi_{i+1 / 2, m}-\phi_{i-1 / 2, m}}{h}+\Sigma_{t} \phi_{i, m}-\Sigma_{s} \sum_{m=1}^{M} w_{m} \phi_{i, m}=\alpha \phi_{i, m} .
$$

where $\phi_{i, m}$ represents the direction $m$ flux in the center of mesh $i$ and $\phi_{i \pm 1 / 2, m}$ represents the direction $m$ flux on the edges of mesh $i$. Eliminating the center fluxes with the diamond difference relation

$$
\phi_{i, m}=\frac{\phi_{i+1 / 2, m}+\phi_{i-1 / 2, m}}{2}
$$

yields the generalized eigenvalue problem of the form

$$
A \vec{\Phi}=\alpha B \vec{\Phi}
$$

where $\vec{\Phi}$ contains the mesh-edge directional fluxes. Solving this problem with standard algorithms yields $N M$ prompt eigenpairs. Due to the discretization of the phase space in these models, $N M$ often greatly exceeds the number of real eigenvalues expected from theory. In this case, the method calculates the real eigenvalues along with an additional spectrum, most of which consists of points within the continuum that are determined by the nature of the discretization. The study shows this method 
is accurate for homogeneous and multi-region slabs with isotropic scattering. It notes that the method is less accurate than semi-analytical methods, but is more versatile for extension to more realistic media. Still, the analysis becomes more complicated with anisotropic scattering, multiple energy groups, and complex geometries.

Method 8: Another study forms a matrix problem from a discretized form of the multigroup, one-dimensional $P_{n}$ equations [58]. It uses a least-squares functional for the first order equation, a spherical harmonics expansion to treat the angular space, and a finite element discretization for position space. The result of these approximations is the discrete $\alpha$-eigenvalue problem

$$
\mathbf{L} \vec{\Phi}+\alpha \mathbf{V} \vec{\Phi}=(\mathbf{S}+\mathbf{F}) \vec{\Phi}
$$

where $\mathbf{M}, \mathbf{V}, \mathbf{S}$, and $\mathbf{F}$ are numerical approximations to the streaming, timeabsorption, scattering, and fission operators, and $\vec{\Phi}$ contains the directional fluxes at nodes. This is similar to an eigenvalue problem using the $S_{n}$ method. To solve for the eigenvalues, the study rearranges the above problem into a standard problem for the inverse eigenvalue

$$
-(\mathbf{L}-\mathbf{S}-\mathbf{F})^{-1} \mathbf{V} \vec{\Phi}=\mathbf{A} \vec{\Phi}=\alpha^{-1} \vec{\Phi}
$$

After finding the eigenvalues of the left product matrices, they are inverted to yield the $\alpha$ eigenvalues. In this formulation, there are two steps to applying the operator $\mathbf{A}$ : multiplying by $\mathbf{V}$ and applying the operator $(\mathbf{L}-\mathbf{S}-\mathbf{F})^{-1}$. The former is just multiplication by a scalar, but the latter is a fixed source problem. The difficulty of solving this problem increases with multigroup and upscattering, leading to slow convergence with a Gauss-Seidel procedure. Also, due to the need to solve a fixedsource problem, the method is applicable only to subcritical systems. The Implicitly Restarted Arnoldi Method (IRAM) determines a fixed number of dominant eigenpairs of $\mathbf{A}$. The result is a set of the desired number of largest $\alpha$ eigenvalues of the system. The study applies this method to homogeneous slabs for verification and to a multigroup slab with ADS geometry. Both applications yield accurate results.

Method 9: This scheme is similar to the previous but is notable for its application to a three-dimensional simplified model of an ADS [59]. The method uses the three-dimensional $S_{n}$ equations with the scatter and fission terms expanded in spherical harmonics and the discontinuous finite element method for position space. This results in a discrete $\alpha$-eigenvalue problem like that in Eq. (2.139), which is subsequently transformed into Eq. (2.140). The IRAM and Sub-space Iteration (SSI) 
methods yield a selected number of dominant eigenvalues and eigenfunctions of the problem. Eigenvalues for a three-dimensional one-group square compare well to analytical solutions and the method successfully finds eigenpairs for the Venus ADS. Due to the similarities to Method 8 and the inversion in Eq. (2.140), it shares most of its characteristics.

\subsubsection{Monte Carlo Methods}

The following methods use Monte Carlo codes but differ slightly in finding eigenpairs. The fixed-source calculation discussed in $\S 2.5 .1$ also uses Monte Carlo codes.

Method 10: The method included in older MCNP versions is a $k$ - $\alpha$ iteration on the prompt $\alpha$ eigenvalue problem given in Eq. (2.51). This iterative method starts with a standard $k$-eigenvalue power iteration [24]:

- Make an initial guess $\alpha^{(0)}$, where usually $\alpha^{(0)}=0$.

- Obtain $k^{(0)}$ and $\phi^{(0)}$ via Monte Carlo power iteration, adjusting the absorption cross section by $\alpha^{(0)} / v$.

- Make a second guess with the eigenvalue modifier $\alpha^{(1)}=\alpha^{(0)}+E V M$.

- Obtain $k^{(n)}$ and $\phi^{(n)}$ via Monte Carlo power iteration, adjusting the absorption cross section by $\alpha^{(n)} / v$.

- Plot the points $\left(k^{(n-1)}, \alpha^{(n-1)}\right)$ and $\left(k^{(n)}, \alpha^{(n)}\right)$ and perform a linear or quadratic extrapolation to determine $\alpha^{(n+1)}$ such that $k(\alpha)=1$.

- Repeat the last two steps until convergence, $k^{(n)}=1$.

The results of this iteration is the prompt fundamental eigenpair because the method ignores delayed neutron precursors. Poor selections of the initial guess and eigenvalue modifier slow the search procedure, and cause failure in some cases for subcritical systems. For early iterations, the root finding procedure for updating the eigenvalue guess causes the search procedure to flounder about. Due to the large computational demand of the Monte Carlo power iteration, some studies developed additional procedures to accelerate the convergence of this $k$ - $\alpha$ iteration [25].

Method 11: The last study presents results using a Monte Carlo power iteration like those described in Eqs. (2.62) and (2.70) for determining the $k$ and $c$ eigenvalues [60]. Based on Eq. (2.51), the prompt $\alpha$-eigenvalue problem for iteration $n$ is

$$
\mathbf{M} \phi_{\alpha}^{(n+1)}=\left(\mathbf{F}-\frac{\alpha_{0}^{(n)}}{v}\right) \phi_{\alpha}^{(n)}
$$

The method must account for the time-absorption term during the Monte Carlo 
random walk [61]: it does so by adjusting the particle weights. This weight change $d W$ of a neutron particle over a flight length $d s$ is

$$
d W=-\frac{\alpha}{v} W d s
$$

Integrating this over the entire path length, 0 to $s$, yields

$$
W(s)=W_{0} \exp \left(-\frac{\alpha}{v} s\right)
$$

where $W_{0}$ is the initial particle weight. Because the weight constantly changes with distance traveled, the product $T_{i}$ of the track length $s_{i}$ and the weight $W(s)$ is an integral of this equation $\int_{0}^{s_{i}}(\cdot) d s$, yielding

$$
T_{i}=W_{0} \frac{v_{i}}{\alpha}\left[1-\exp \left(-\frac{\alpha}{v_{i}} s_{i}\right)\right] .
$$

A track length estimator obtains $\alpha^{(n+1)}$ during the random walk

$$
\alpha_{0}^{(n+1)}=\alpha_{0}^{(n)}\left[1+\frac{1}{\sum_{i} \Delta W_{i}}\left(N-\sum_{i} \bar{\nu}_{p} \Sigma_{f} T_{i}\right)\right]
$$

where the sums are over all trajectories, and

$$
\Delta W_{i}=W_{0}\left[1-\exp \left(-\frac{\alpha}{v_{i}} s_{i}\right)\right]
$$

Additionally, at each fission site, the number of neutrons stored changes to

$$
\left\lfloor\frac{W \bar{\nu}_{p} \sigma_{f}}{\sigma_{t}}+\xi\right\rfloor
$$

where $\bar{\nu}_{p}$ is the average number of prompt neutrons released per fission. This is Eq. (2.65) without the $k_{0}^{(n)}$. The final result is the prompt fundamental eigenpair. This method uses a weight cancellation scheme to obtain the next eigenpair and a elimination method to obtain the third and fourth eigenpairs. The latter method is 
similar to that described in Method 3, except the coefficients are

$$
\begin{array}{r}
a_{i}=\frac{\left\langle\phi_{i}^{\dagger}, v^{-1} \phi\right\rangle}{\left\langle\phi_{i}^{\dagger}, v^{-1} \phi_{i}\right\rangle}, \\
b_{i}=\frac{\left\langle\phi, v^{-1} \phi_{i}^{\dagger}\right\rangle}{\left\langle\phi_{i}, v^{-1} \phi_{i}^{\dagger}\right\rangle} .
\end{array}
$$

The first three calculated eigenvalues compare well to fixed-source calculations of the decay constants. For higher modes, the fixed-source calculations define geometries and sources such that the higher modes are excited for an extended period of time.

For very subcritical systems, $\alpha<0$, where the time-absorption term dominates the fission source, the method subtracts a $\lambda \alpha / v$ term from both sides of the equation. The additional term on the left side of the equation increases the total cross section, leading to more absorptions. To perform the power iteration with this adjustment, replace $\alpha$ with

$$
\alpha^{\prime}=(1+\lambda) \alpha
$$

in the previous formulations. This shows better numerical stability for subcritical problems.

Upon close inspection, this method is very much like the $k$ - $\alpha$ iteration. The number of neutrons stored at each fission site described in Eq. (2.147) is the same as those stored for a critical $k$-eigenvalue calculation, where $k_{0}^{(n)}=1$. The weight adjustment described in Eq. (2.143) is just implicit absorption along a flight path, where $\alpha / v$ is the absorption cross section. These procedures are similar to a $k$ eigenvalue Monte Carlo calculation, with a tally to update $\alpha$ for the next cycle.

\subsubsection{Summary}

These eleven methods provide a general overview of the basic principles and results yielded from current techniques for calculating $\alpha$ eigenvalues (Table 2.4). These methods are classifiable in a number of ways. Some methods use iterative procedures $(2,3,5,10$, and 11), while some are based on a matrix formulation $(4,6,7,8$, and 9$)$. Some treat extremely simplified systems to obtain a better theoretical understanding of the spectrum of the linear transport operator $(5,6$, and 7$)$, while others pursue schemes that obtain dominant kinetic modes of multi-dimensional reactor models (4 and 9). Some are designed specifically to treat purely-scattering media (5 and 7), 
Table 2.4: Summary of methods for calculating $\alpha$ eigenvalues, eigenfunctions, and kinetic modes.

\begin{tabular}{|c|c|c|}
\hline Method & Type & Results \\
\hline 1-FS & $\begin{array}{l}\text { MC Pulsed-Source Calculation } \\
\text { with Flux Time Response Fit }\end{array}$ & $\begin{array}{l}\text { Prompt Fundamental Eigenvalue, } \\
\text { Limited Kinetic Mode Information }\end{array}$ \\
\hline 2-Diff. & $\begin{array}{l}k \text { - } \alpha \text { Iteration with Modified } \\
\text { Inhour Eq. }\end{array}$ & Delayed Fundamental Eigenpair \\
\hline 3-Diff. & $\begin{array}{l}k \text { - } \alpha \text { Iteration with Elimination } \\
\text { Method }\end{array}$ & $\begin{array}{l}\text { Several Higher Delayed and Prompt } \\
\text { Eigenpairs }\end{array}$ \\
\hline 4-Diff. & $\begin{array}{l}\text { Homogeneous Reactor Mode } \\
\text { Expansion Matrix Method }\end{array}$ & $\begin{array}{l}J \text { Delayed and } 60-100 \text { Prompt } \\
\text { Eigenpairs of FSV }\end{array}$ \\
\hline 5-Trans. & $\begin{array}{l}c \text {-Eigenvalue Critical Thickness } \\
\text { Iteration }\end{array}$ & $\begin{array}{l}\text { Real Eigenvalue Spectrum of } \\
\text { One-Speed, Homogeneous Slabs and } \\
\text { Spheres }\end{array}$ \\
\hline 6-Trans. & Green's Function Method & $\begin{array}{l}\text { Real Eigenpairs of One-Speed, } \\
\text { Multi-Region Slabs }\end{array}$ \\
\hline 7-Trans. & $\begin{array}{l}\text { Discrete Ordinates, Finite } \\
\text { Difference Matrix Method }\end{array}$ & $\begin{array}{l}\text { Real and Complex Eigenpairs of } \\
\text { One-Speed, Multi-Region Slabs }\end{array}$ \\
\hline 8-Trans. & $\begin{array}{l}\text { Spherical Harmonic, Finite } \\
\text { Element Matrix Method }\end{array}$ & $\begin{array}{l}\text { Dominant Prompt Eigenpairs of } \\
\text { Multigroup Slabs }\end{array}$ \\
\hline 9-Trans. & $\begin{array}{l}\text { Discrete Ordinates, Finite } \\
\text { Element Matrix Method }\end{array}$ & $\begin{array}{l}\text { Dominant Prompt Eigenpairs of an } \\
\text { ADS }\end{array}$ \\
\hline $10-\mathrm{MC}$ & $k$ - $\alpha$ Iteration & Prompt Fundamental Eigenpair \\
\hline $11-\mathrm{MC}$ & $\begin{array}{l}\text { Power Iteration with Weight } \\
\text { Cancellation and Elimination } \\
\text { Method }\end{array}$ & Few Prompt Eigenpairs \\
\hline
\end{tabular}

while others treat subcritical $(1,8$, and 9) or supercritical (10) media. The TRMM bridges the gap between some of these divisions: it is a Monte Carlo matrix method that treats any scattering or multiplying media, from simplified to complex.

Time-dependent fixed-source calculations (method 1) are inefficient for complex geometries and far subcritical assemblies. The example using MCNP for a subcritical configuration of the FSV reactor shows results achieved with a very long runtime and several variance reduction techniques. Even with these measures, it is still difficult to quantify the delayed neutron background. Furthermore, the best possible results are only the first few eigenvalues and some spatial kinetic mode information.

Iterative techniques (methods $2,3,5,10$, and 11) are easily integrable into exist- 
ing criticality codes: the methods are essentially repeated criticality calculations with an adjusted total cross section and emission spectrum. While the efficiency of the criticality code and the method of updating $\alpha$ determine the overall speed of these methods, the iteration must be repeated to obtain different eigenvalues. It is easy to see cases where obtaining several eigenvalues comes at a large computational cost. Additionally, the treatment of the time-absorption term causes numerical difficulties in some cases. The time-absorption term combines with the total cross section, resulting in an adjusted effective cross section, $\Sigma_{\text {eff }}=\alpha / v+\Sigma$. If $\alpha>0$, this yields a larger effective cross section. If $\alpha<0$ and $|\alpha / v|<\Sigma$, this yields a smaller effective cross section. But, if $\alpha<0$ and $|\alpha / v|>\Sigma$, then the cross section becomes negative, $\Sigma_{\text {eff }}<0$, which is unphysical. The time-absorption term then becomes a source term, and as $\alpha$ becomes increasingly negative, the term dominates the fission source,

causing problems with transport calculations. While the technique of subtracting the extra $\lambda \alpha / v$ term from both sides of the equation attempts to addresses this issue, ultimately, some of these methods operate well for supercritical and near-critical systems, but struggle for far subcritical systems.

The matrix formulations (methods $4,6,7,8,9$ ) are most similar to the TRMM due to the treatment of the phase space and the building of the $\alpha$-eigenvalue matrix problem. The calculated eigenvalue spectra of these methods are similar, including the calculation of point spectra within the continuum. Some of these methods require a solution to a fixed-source problem, which comes at a computational cost and limits their application to certain problems.

\section{References}

[1] G. I. Bell and S. Glasstone, Nuclear Reactor Theory. New York: Van Nostrand Reinhold Company, 1970.

[2] J. J. Duderstadt and L. J. Hamilton, Nuclear Reactor Analysis. New York: John Wiley \& Sons, 1976.

[3] K. O. Ott and D. A. Menely, Introductory Nuclear Reactor Dynamics. La Grange Park, Illinois: American Nuclear Society, 1985.

[4] E. W. Larsen and P. F. Zweifel, "On the Spectrum of the Linear Transport Operator," Journal of Mathematical Physics, vol. 15, pp. 1987-1997, 1974.

[5] M. B. Chadwick, P. Obložinký, M. Herman, et al., "ENDF/B-VII.0: Next Generation Evaluated Nuclear Data Library for Nuclear Science and Technology,", Nucl. Data Sheets. 107, pp. 2931-3060, 2006.

[6] E. E. Lewis and W. F. Miller, Computational Methods of Neutron Transport. New York: John Wiley \& Sons, 1984. 
[7] M. Ash, Nuclear Reactor Kinetics. New York: McGraw-Hill, 1965.

[8] J. J. Duderstadt and W. R. Martin, Transport Theory. New York: John Wiley \& Sons, 1979.

[9] Y. Cao, Space-Time Kinetics and Time-Eigenfunctions. PhD thesis, University of Michigan, 2008.

[10] T. M. Sutton and B. N. Aviles, "Diffusion Theory Methods for Spatial Kinetics Calculations," Progress in Nuclear Energy, vol. 30, pp. 119-182, 1996.

[11] J. B. Andrews II and K. F. Hansen, "Numerical Solution of the Time-Dependent Multigroup Diffusion Equations," Nuclear Science and Engineering, vol. 31, pp. 304-313, 1968.

[12] B. D. Ganapol, "Solution of the One-Group Time-Dependent Neutron Transport Equation in an Infinite Medium by Polynomial Reconstruction," Nuclear Science and Engineering, vol. 92, pp. 272-279, 1986.

[13] N. R. Corngold, "Time-Dependent, One-Speed Transport via Generalized Functions," Nuclear Science and Engineering, vol. 137, pp. 352-358, 2001.

[14] A. F. Henry, "The Application of Reactor Kinetics to the Analysis of Experiments," Nuclear Science and Engineering, vol. 3, pp. 52-70, 1958.

[15] S. Dulla, E. H. Mund, and P. Ravetto, "The Quasi-static Method Revisited," Progress in Nuclear Energy, vol. 50, pp. 908-920, 2008.

[16] K. O. Ott and D. A. Menely, "Accuracy of the Quasistatic Treatment of Spatial Reactor Kinetics," Nuclear Science and Engineering, vol. 36, pp. 402-411, 1969.

[17] S. Kaplan, O. J. Marlowe, and J. Bewick, "Application of Synthesis Techniques to Problems Involving Time Dependence," Nuclear Science and Engineering, vol. 18, pp. 163-176, 1964.

[18] J. B. Yasinsky and A. F. Henry, "Some Numerical Experiments Concerning Space-Time Reactor Kinetics Behavior," Nuclear Science and Engineering, vol. 22, pp. 171-181, 1965.

[19] L. L. Carter and E. D. Cashwell, "Particle-Transport Simulation with the Monte Carlo Method," Tech. Rep. TID-26607, Los Alamos National Laboratory, 1975.

[20] E. W. Larsen, "NERS 543: Advanced Nuclear Reactor Theory." University of Michigan, Lecture Notes, Fall, 2008.

[21] B. L. Sjenitzer and J. E. Hoogenboom, "A Monte Carlo Method for Calculation on the Dynamic Behaviour of Nuclear Reactors," in Proc. Joint International Conference on Supercomputing in Nuclear Applications and Monte Carlo 2010, (Tokyo, Japan), October 2010.

[22] G. W. McKinney, "MCNP6 Enhancements of Delayed-Particle Production," in Proc. PHYSOR 2012 Advances in Reactor Physics Linking Research, Industry, and Education, (Knoxville, Tennessee), April 2012.

[23] B. L. Sjenitzer and J. E. Hoogenboom, "General Purpose Dynamic Monte Carlo with Continuous Energy for Transient Analysis," in Proc. PHYSOR 2012 Advances in Reactor Physics Linking Research, Industry, and Education, (Knoxville, Tennessee), April 2012.

[24] D. Brockway, P. Soran, and P. Whalen, "Monte-Carlo Eigenvalue Calculation." Los Alamos National Laboratory, 1985.

[25] T. R. Hill, "Efficient Methods for Time Absorption $(\alpha)$ Eigenvalue Calculations," 
Tech. Rep. LA-9602-MS, Los Alamos National Laboratory, 1983.

[26] D. G. Cacuci, Y. Ronen, Z. Shayer, J. J. Wagschal, and Y. Yeivin, "EigenvalueDependent Neutron Energy Spectra: Definitions, Analyses, and Applications," Nuclear Science and Engineering, vol. 81, pp. 432-442, 1982.

[27] G. Velarde, C. Ahnert, and J. M. Aragonés, "Analysis of the Eigenvalue Equations in $k, \lambda, \gamma$, and $\alpha$ Applied to Some Fast- and Thermal-Neutron Systems," Nuclear Science and Engineering, vol. 66, pp. 284-294, 1978.

[28] F. Brown, "Fundamentals of Monte Carlo Particle Transport," Tech. Rep. LAUR-05-4983, Los Alamos National Laboratory, 2005.

[29] Y. Ronen, D. Shvarts, and J. J. Wagschal, "A Comparison of Some Eigenvalues in Reactor Theory," Nuclear Science and Engineering, vol. 60, pp. 97-101, 1976.

[30] E. Dumonteil and T. Courau, "Dominance Ratio Assessment and Monte Carlo Criticality Simulations: Dealing with High Dominance Ratio Systems," Nuclear Technology, vol. 172, pp. 120-131, 2009.

[31] M. Andoh, T. Misawa, and K. Nishina, "Measurement of Flux Tilt and Eigenvalue Separation in Axially Decoupled Core," Nuclear Science and Technology, vol. 34, pp. 445-453, 1997.

[32] S. E. Carney, F. B. Brown, B. C. Kiedrowski, and W. R. Martin, "Fission Matrix Capability for MCNP, Part II - Applications," Tech. Rep. LA-UR-13-20454, Los Alamos National Laboratory, 2013.

[33] D. E. Kornreich and D. K. Parsons, "Time-eigenvalue Calculations in Multiregion Cartesian Geometry Using Green's Functions," Annals of Nuclear Energy, vol. 32, pp. 964-985, 2005.

[34] X-5 Monte Carlo Team, "MCNP - A General N-Particle Transport Code, Version 5, Volume I: Overview and Theory," Tech. Rep. LA-UR-03-1987, Los Alamos National Laboratory, 2003.

[35] B. C. Kiedrowski, "Evaluation of Computing c-Eigenvalues with Monte Carlo," Tech. Rep. LA-UR-12-00577, Los Alamos National Laboratory, 2012.

[36] M. Nelkin, "Asymptotic Solutions of the Transport Equation for Thermal Neutrons," Physica, vol. 29, pp. 261-273, 1963.

[37] J. Lehner and G. M. Wing, "On the Spectrum of an Unsymmetric Operator Arising in the Transport Theory of Neutrons," Communications on Pure and Applied Mathematics, vol. 8, pp. 217-234, 1955.

[38] K. Jörgens, "An Asymptotic Expansion in the Theory of Neutron Transport," Communications on Pure and Applied Mathematics, vol. 11, pp. 219-242, 1958.

[39] A. E. Taylor, Introduction to Functional Analysis. New York: John Wiley \& Sons, 1958.

[40] G. M. Wing, An Introduction to Transport Theory. New York: John Wiley \& Sons, 1962.

[41] W. Pfeiffer, J. R. Brown, and A. C. Marshall, "Analysis and Results of PulsedNeutron Experiments on the Fort St. Vrain HTGR," Tech. Rep. GA-A13342, General Atomic, 1975.

[42] J. Lehner and G. M. Wing, "Solution of the Linearized Boltzmann Transport Equation for the Slab Geometry," Duke Mathematical Journal, vol. 23, pp. 125$142,1956$. 
[43] Y. Cao and J. C. Lee, "Spatial Corrections for Pulsed-Neutron Reactivity Measurements," Nuclear Science and Engineering, vol. 165, pp. 270-282, 2010.

[44] W. Pfeiffer, J. R. Brown, and A. C. Marshall, "Fort St. Vrain Startup Test A-3: Pulsed-Neutron Experiments," Tech. Rep. GA-A13079, General Atomic, 1974.

[45] C. P. Baker, "Time Scale Measurements by the Rossi Method," Tech. Rep. LA617, Los Alamos National Laboratory, 1947.

[46] B. Richard, "Neutron Noise Measurements on Fast Burst Reactor CALIBAN Determination of the Core Kinetic Parameters," Nuclear Science and Engineering. Accepted for Publication.

[47] G. D. Spriggs, "Two Rossi- $\alpha$ Techniques for Measuring the Effective Delayed Neutron Fraction," Nuclear Science and Engineering, vol. 113, pp. 161-172, 1993.

[48] M. Sohrabpour and A. O. Ezzati, "Monte Carlo Simulation and Benchmarking of Pulsed Neutron Experiments in Variable Buckling Beo Systems," Annals of Nuclear Energy, vol. 36, pp. 547-549, 2008.

[49] Z. Li, X. Yang, T. Li, G. Yu, and K. Wang, "Monte Carlo Simulations and Experimental Validations of $\alpha$ Eigenvalues," in Proc. Joint International Conference on Supercomputing in Nuclear Applications and Monte Carlo 2010, (Tokyo, Japan), October 2010.

[50] B. R. Betzler, J. C. Lee, and W. R. Martin, "MCNP5 Analysis of Fort St. Vrain High-Temperature Gas-Cooled Reactor," in Trans. Am. Nucl. Soc., vol. 102, p. 515, 2010.

[51] W. Pfeiffer, "FRNTIC - A Computer Code for Weighted Least Squares Analysis of Pulsed-Neutron Data," Tech. Rep. GA-A13077, General Atomic, 1974.

[52] J. E. Hoogenboom, "Numerical Calculation of the Delayed- $\alpha$ Eigenvalue Using a Standard Criticality Code," in Proc. PHYSOR 2002 International Conference on the New Frontiers of Nuclear Technology, (Seoul, South Korea), October 2002.

[53] K. P. Singh, S. B. Degweker, R. S. Modak, and K. Singh, "Iterative Method for Obtaining the Prompt and Delayed Alpha-modes of the Diffusion Equation," Annals of Nuclear Energy, vol. 38, pp. 1996-2004, 2011.

[54] W. Pfeiffer, "Computer Simulation of Pulsed-Neutron Experiments for the Fort St. Vrain HTGR," Tech. Rep. Gulf-GA-B10959, Gulf General Atomic, 1972.

[55] W. Pfeiffer, "RTMODE and RZMODE - Two-Dimensional Computer Codes for Simulating Pulsed-Neutron Experiments," Tech. Rep. Gulf-GA-B10877, Gulf General Atomic, 1971.

[56] E. B. Dahl, V. Protopopescu, and N. G. Sjöstrand, "On the Relation Between Decay Constants and Critical Parameters in Monoenergetic Neutron Transport," Nuclear Science and Engineering, vol. 83, pp. 374-379, 1983.

[57] R. S. Modak and A. Gupta, "A Simple Scheme for the Direct Evaluation of the Time-Eigenvalues of Neutron Transport Equation," Annals of Nuclear Energy, vol. 30, pp. 211-222, 2003.

[58] D. Lathouwers, "Iterative Computation of Time-eigenvalues of the Neutron Transport Equation," Annals of Nuclear Energy, vol. 30, pp. 1793-1806, 2003.

[59] J. Kópházi and D. Lathouwers, "Three-dimensional Transport Calculation of Multiple Alpha Modes in Subcritical Systems," Annals of Nuclear Energy, vol. 50, pp. 167-174, 2012. 
[60] T. Yamamoto, "Higher Order $\alpha$ Mode Eigenvalue Calculation by Monte Carlo Power Iteration," Progress in Nuclear Science and Technology, vol. 2, pp. 826835, 2011.

[61] T. Yamamoto and Y. Miyoshi, "An Algorithm of $\alpha$ - and $\gamma$-Mode eigenvalue Calculations by Monte Carlo Method," Tech. Rep. JAERI-Conf2003-019, Japan Atomic Energy Research Institute, 2003. 


\section{CHAPTER 3}

\section{The Markov Transition Rate Matrix Method}

A convenient way of modeling a stochastic process is to build a mathematical representation of the behavior the system by classifying a collection of states that the system possibly occupies and defining how it moves among these states. Transitions from one state to another define the evolution of the process. Markov processes are a class of stochastic process that satisfy the Markov property: the probability that the physical system is in a given state at time $t_{i+1}$ is deduced from a knowledge of its state at any earlier time $t_{i}$, and does not depend on the history of the system before time $t_{i}[1]$. Examples of Markov processes extend to several fields of study, including biology, social sciences, business, and engineering: for the formulation of the Transition Rate Matrix Method (TRMM), neutron transport is recognized as one of these examples.

The Transition Rate Matrix (TRM) is the operator of the adjoint $\alpha$-eigenvalue equation, obtained from a formulation using the time-dependent neutron importance equation: the equation adjoint to the neutron transport equation. Solutions to the adjoint equation have a physical significance as the importance of neutrons within a the system [2]. The TRM identifies a set of states and defines how transitions occur between the states: it describes a Markov process. A forward Monte Carlo calculation tallies estimates of the elements of the TRM, and eigenvalue solvers determine the eigenpairs of the matrix: these are estimates of the adjoint $\alpha$ eigenpairs of the underlying system.

This chapter describes the Markov TRMM process of approximating $\alpha$ eigenpairs of scattering and multiplying media and the use of this information to build the time-dependent expansion solution to arbitrary sources. Because Monte Carlo treats the phase space as continuous, the angular variable $\hat{\Omega}$ is reintroduced into the following formulations: this variable is important to the functionality of the method. This chapter discusses Markov processes, the neutron importance equation, the the- 
oretical formulation of the TRMM, the relationship between the forward and adjoint $\alpha$-eigenvalue problems, the similarities between the TRMM and the fission matrix method, derivations of the TRMM for simplified media, Monte Carlo transition rate tallies, and eigenvalue solvers.

\subsection{Theoretical Formulation}

The formulation of the TRMM relies on the interpretation that the operator in the adjoint $\alpha$-eigenvalue problem describes a continuous-time Markov process: elements of this operator are rates defining neutrons transitioning among the position-energydirection phase space. After obtaining the adjoint operator, subsequent matrix manipulations yield the forward operator. Together, solutions to these operators provide estimates of the adjoint and forward $\alpha$ eigenpairs: this is all the information necessary to perform eigenfunction expansion.

\subsubsection{Markov Processes}

A Markov process is the set of random variables $\{X(t), t \in T\}$, where $X(t)$ are states, $t$ is the parameter, and $T$ describes the parameter space. Often, the parameter is time, so $X(t)$ denotes the state assumed at time $t$. The nature of the parameter and state spaces define four classifications of Markov processes. If the parameter space is discrete, i.e., $T=\{0,1,2, \cdots\}$, then it is a discrete-parameter Markov

process. If the parameter space is continuous, i.e., $T=\{0 \leq t<\infty\}$, then it is a continuous-parameter Markov process. Likewise, if the state space is discrete, i.e., $X(t)=\left\{X_{0}, X_{1}, X_{2}, \cdots\right\}$, then it is a Markov chain. If the state space is continuous, i.e., $X(t)=\{0 \leq X(t)<\infty\}$, then it is a Markov process [3].

Neutron transport is a Markov process, where the parameter is time and the states are within the position-energy-direction phase space. Both the state and parameter spaces are continuous, so transport is a continuous-time Markov process. But, for the ease of computation, the state space is made finite, and it becomes a continuous-time Markov chain.

Continuous-parameter Markov chains are characterized by the transition intensity, or transition rate, matrix

$$
\mathbf{Q}=\left[\begin{array}{ccc}
-q_{1} & q_{12} & \cdots \\
q_{21} & -q_{2} & \cdots \\
\vdots & \vdots & \ddots
\end{array}\right]
$$


The diagonal quantities, $q_{i}$, are the intensity of passage, given that the Markov process is in state $i$. The off-diagonal quantities, $q_{i j}$ are the intensity of transition to $j$, given that the Markov process is in state $i$ [4]. In relation to the transition probability $p_{i j}$, the intensities are

$$
q_{i j}=\lim _{\Delta t \rightarrow 0}\left\{\frac{p_{i j}(t, t+\Delta t)}{\Delta t}\right\}, \quad \text { for } \quad i \neq j
$$

where $\Delta t$ is the period of observation and $p_{i j}(t, t+\Delta t)$ is the probability that a transition occurs in the interval $[t, t+\Delta t)$. It is common to normalize the transition intensities such that

$$
q_{i}=\sum_{j \neq i} q_{i j}
$$

but this is unnecessary in cases where an absorbing state is left undefined. For a purely absorbing state, $q_{i}=0$. The time that passes while transitioning between states is negligible, i.e., transitions between states occur instantaneously.

In the application to neutron transport, the entrants of the $\mathbf{Q}$ matrix are transition rates between states, where $q_{i}$ is the removal rate from state $i$ and $q_{i, j}$ is the the transition rate from state $i$ to state $j$. By defining discrete states of the physical system as specific conditions that neutrons meet in the position-energy-direction phase space, the $\mathbf{Q}$ matrix characterizes the behavior of neutrons in the system. Monte Carlo is well-suited to obtaining the transition rates in $\mathbf{Q}$ because it generates many individual neutron tracks, tallying average quantities for transition rates out of and between states. Conveniently, this $\mathbf{Q}$ matrix describing neutron transport is the operator of the adjoint $\alpha$-eigenvalue problem. The entries of the operator resemble the transition rates characterizing a continuous-time Markov process: with the $\mathbf{Q}$ matrix, the adjoint $\alpha$-eigenvalue spectrum is known.

\subsubsection{The Time-Dependent Neutron Importance Equation}

The time-dependent neutron transport and precursor equations in operator form are

$$
\begin{aligned}
\frac{1}{v} \frac{\partial \psi}{\partial t}+\mathbf{M} \psi(\mathbf{r}, E, \hat{\mathbf{\Omega}}, t) & =\mathbf{F}_{\mathbf{p}} \psi+\sum_{j}^{J} \frac{\chi_{j}(E)}{4 \pi} \lambda_{j} C_{j}, \\
\frac{\partial C_{j}}{\partial t}+\lambda_{j} C_{j}(\mathbf{r}, t) & =\mathbf{F}_{\mathbf{d} j} \psi, \quad \text { for } \quad j=1, \ldots, J,
\end{aligned}
$$

where the operators are as defined in Eqs. (2.3) through (2.8). These equations describes the balance of neutrons within the system, with the additional $J$ delayed 
precursor equations included to simplify the neutron transport equation.

The time-dependent neutron importance, or fundamental adjoint, equations are

$$
\begin{aligned}
-\frac{1}{v} \frac{\partial \psi^{\dagger}}{\partial t}+ & \mathbf{M}^{\dagger} \psi^{\dagger}(\mathbf{r}, E, \hat{\mathbf{\Omega}}, t)=\mathbf{F}_{\mathbf{p}}^{\dagger} \psi^{\dagger}+\sum_{j}^{J} \mathbf{F}_{\mathbf{d}_{j}^{\dagger} C_{j}^{\dagger}} \\
-\frac{\partial C_{j}^{\dagger}}{\partial t}+\lambda_{j} C_{j}^{\dagger}(\mathbf{r}, t) & =\left(\chi_{j} \lambda_{j}\right)^{\dagger} \psi^{\dagger}, \quad \text { for } \quad j=1, \ldots, J
\end{aligned}
$$

where the adjoint operators are

$$
\begin{aligned}
\mathbf{L}^{\dagger} \psi^{\dagger} & =-\hat{\mathbf{\Omega}} \cdot \nabla \psi^{\dagger} \\
\mathbf{R}^{\dagger} \psi^{\dagger} & =\Sigma(\mathbf{r}, E) \psi^{\dagger} \\
\mathbf{S}^{\dagger} \psi^{\dagger} & =\iint \Sigma_{s}\left(\mathbf{r} ; E, \hat{\mathbf{\Omega}} \rightarrow E^{\prime}, \hat{\mathbf{\Omega}}^{\prime}\right) \psi^{\dagger}\left(\mathbf{r}, E^{\prime}, \hat{\mathbf{\Omega}}^{\prime}, t\right) d E^{\prime} d \mathbf{\Omega}^{\prime}, \\
\mathbf{M}^{\dagger} \psi^{\dagger} & =\mathbf{L}^{\dagger} \psi^{\dagger}+\mathbf{R}^{\dagger} \psi^{\dagger}-\mathbf{S}^{\dagger} \psi^{\dagger} \\
\mathbf{F}_{\mathbf{p}}^{\dagger} \psi^{\dagger} & =\iint(1-\beta) \bar{\nu}(E) \Sigma_{f}(\mathbf{r}, E) \frac{\chi_{p}\left(E^{\prime}\right)}{4 \pi} \psi^{\dagger}\left(\mathbf{r}, E^{\prime}, \hat{\mathbf{\Omega}}^{\prime}, t\right) d E^{\prime} d \mathbf{\Omega}^{\prime}, \\
\mathbf{F}_{\mathbf{d}_{j}^{\dagger} C_{j}^{\dagger}} & =\beta_{j} \bar{\nu}(E) \Sigma_{f}(\mathbf{r}, E) C_{j}^{\dagger}(\mathbf{r}, t), \\
\left(\chi_{j} \lambda_{j}\right)^{\dagger} \psi^{\dagger} & =\iint \frac{\chi_{j}\left(E^{\prime}\right)}{4 \pi} \lambda_{j} \psi^{\dagger}\left(\mathbf{r}, E^{\prime}, \hat{\mathbf{\Omega}}^{\prime}, t\right) d E^{\prime} d \mathbf{\Omega}^{\prime}
\end{aligned}
$$

where $\psi^{\dagger}$ is the adjoint angular flux and $C_{j}^{\dagger}$ is the adjoint precursor concentration, and $\left(\chi_{j} \lambda_{j}\right)^{\dagger}$ is the adjoint precursor emission operator. These equations describe the importance of neutrons within the system, with the adjoint delayed neutron precursor equations serving a similar role as for the forward neutron transport equation. Give extra attention to the differences in the forward and adjoint operators as described in these two formulations: (i) in the adjoint equations, the time derivative term is negative; (ii) in the adjoint equations, the streaming term $\hat{\Omega} \cdot \nabla$ has the opposite sign; (iii) the delayed fission operator has no integral; (iv) and the adjoint formulation swaps the energy and direction variables in the integrals.

\section{Requirement of the Adjoint Operators}

Mathematically, the adjoint operators satisfy the condition

$$
\left\langle\psi^{\dagger}, \mathbf{M} \psi\right\rangle=\left\langle\psi, \mathbf{M}^{\dagger} \psi^{\dagger}\right\rangle
$$


where $\psi(\mathbf{r}, E, \hat{\mathbf{\Omega}})=0$ for all incoming neutron directions on the boundary, $\hat{n} \cdot \hat{\mathbf{\Omega}}<0$, and $\psi^{\dagger}(\mathbf{r}, E, \hat{\Omega})=0$ for all outgoing directions on the boundary, $\hat{n} \cdot \hat{\Omega}>0$ [2]. The operators in Eqs. (3.8) through (3.13) satisfy this requirement. For the collision operator $\mathbf{R}^{\dagger}$, both sides of Eq. (3.15) are identical. For the integral operators, changing variables names reveals equality. For example, inserting the forward and adjoint delayed fission operators into Eq. (3.15) yields

$$
\left\langle C_{j}^{\dagger}, \mathbf{F}_{\mathbf{d} j} \psi\right\rangle=\left\langle\psi, \mathbf{F}_{\mathbf{d}_{j}^{\dagger}}^{\dagger} C_{j}^{\dagger}\right\rangle
$$

Explicitly writing out the operators and integrals yields

$$
\begin{aligned}
\int C_{j}^{\dagger}(\mathbf{r}) d V \iint \beta_{j} \bar{\nu}\left(E^{\prime}\right) \Sigma_{f}\left(\mathbf{r}, E^{\prime}\right) \psi^{\prime} d E^{\prime} d \mathbf{\Omega}^{\prime} & \\
& =\iiint \psi \beta_{j} \bar{\nu}(E) \Sigma_{f}(\mathbf{r}, E) C_{j}^{\dagger}(\mathbf{r}) d E d \boldsymbol{\Omega} d V
\end{aligned}
$$

With some algebra, it is apparent that the integrated quantities are equal. It is less apparent that the leakage operator $\mathbf{L}^{\dagger}$ fulfils this requirement. Moving both terms in Eq. (3.15) to the same side, inserting the operators, and writing out the integrals yields

$$
\iiint \psi^{\dagger} \hat{\boldsymbol{\Omega}} \cdot \nabla \psi d E d \boldsymbol{\Omega} d V+\iiint \psi \hat{\boldsymbol{\Omega}} \cdot \nabla \psi^{\dagger} d E d \boldsymbol{\Omega} d V=0 .
$$

Because the gradient does not act upon direction,

$$
\hat{\Omega} \cdot \nabla \psi=\nabla \cdot \hat{\Omega} \psi
$$

and the integrals are rewritten as

$$
\iiint \nabla \cdot \hat{\mathbf{\Omega}} \psi^{\dagger} \psi d E d \boldsymbol{\Omega} d V=0
$$

Using the divergence theorem, an integral over the surface replaces the volume integral

$$
\int_{\partial V} \iint \hat{n} \cdot \hat{\boldsymbol{\Omega}} \psi^{\dagger} \psi d E d \boldsymbol{\Omega} d S=0
$$

This integral is zero due to the boundary conditions of the forward and adjoint fluxes: the incoming angular and outgoing adjoint angular fluxes are zero, so the multiplicative result $\psi^{\dagger} \psi$ is zero across the surface. 


\section{Physical Interpretation of the Adjoint Flux}

The adjoint angular flux is a measure of neutron importance of arbitrary units. To understand its definition, consider a system with a detector defined by the macroscopic cross section $\sigma_{d}(\mathbf{r}, E, t)$ of the detecting nuclide. Then, the neutron importance $\psi^{\dagger}(\mathbf{r}, E, \hat{\boldsymbol{\Omega}}, t)$ is the future expected response generated in the detector by a neutron at $\mathbf{r}, E, \hat{\boldsymbol{\Omega}}, t$. This includes all secondary neutrons, e.g., from fission or scattering, produced by this neutron [2]. A neutron exiting the boundary of the system does not contribute to the future expected detector response: the importance of outgoing neutrons at a system boundary is zero.

A typical neutron transport problem defines an initial value problem at $t=0$, and uses the transport equation to obtain the angular flux at subsequent times. For neutron importance, this is reversed: a final value problem defined at $t=t_{f}$ uses the fundamental adjoint equation to obtain the importance at earlier times. For example, a change in the detector at a particular time only affects the importance for all previous times: this is opposite to neutron flux. This behavior is due to the opposite sign of the time derivatives in the adjoint equations. Mathematically, this affects the formulation of the adjoint $\alpha$-eigenvalue problem by changing the time dependence in the shape and time function separation. In the forward problem, the time function is a positive exponential. In the adjoint problem, the separations are

$$
\begin{aligned}
\psi^{\dagger}(\mathbf{r}, E, \hat{\mathbf{\Omega}}, t) & =\psi^{\dagger}(\mathbf{r}, E, \hat{\mathbf{\Omega}}) \exp \left(-\alpha^{\dagger} t\right), \\
C_{j}^{\dagger}(\mathbf{r}, t) & =C_{j}^{\dagger}(\mathbf{r}) \exp \left(-\alpha^{\dagger} t\right), \quad \text { for } \quad j=1, \ldots, J,
\end{aligned}
$$

where the adjoint eigenvalues $\alpha^{\dagger}$ follow the trend in Eq. (2.75) because they are the same as the forward eigenvalues $\alpha$. Thus, in a supercritical system, the importance decreases with time, and in a subcritical system, the importance increases with time [2]. This is in line with the physical definition of neutron importance: in a supercritical system, an earlier neutron has more importance because it has additional time to multiply and contribute more to the detector response.

\subsubsection{The Transition Rate Matrix}

The formulation of the adjoint $\alpha$-eigenvalue problem follows the same procedures as the forward formulation described in $\S 2.3 .1$. Following these procedures with Eqs. (3.4) through (3.7) and rearranging terms yields the forward $\alpha$-eigenvalue prob- 
lem

$$
\begin{aligned}
\left(-\mathbf{M}+\mathbf{F}_{\mathbf{p}}\right) \psi+\sum_{j}^{J} \frac{\chi_{j}(E)}{4 \pi} \lambda_{j} C_{j} & =\frac{\alpha}{v} \psi, \\
\mathbf{F}_{\mathbf{d} j} \psi-\lambda_{j} C_{j} & =\alpha C_{j}, \quad \text { for } \quad j=1, \ldots, J,
\end{aligned}
$$

and the adjoint $\alpha$-eigenvalue problem

$$
\begin{aligned}
\left(-\mathbf{M}^{\dagger}+\mathbf{F}_{\mathbf{p}}^{\dagger}\right) \psi^{\dagger}+\sum_{j}^{J} \mathbf{F}_{\mathbf{d}_{j}^{\dagger}} C_{j}^{\dagger} & =\frac{\alpha^{\dagger}}{v} \psi^{\dagger}, \\
\left(\chi_{j} \lambda_{j}\right)^{\dagger} \psi^{\dagger}-\lambda_{j} C_{j}^{\dagger} & =\alpha^{\dagger} C_{j}^{\dagger}, \text { for } j=1, \ldots, J
\end{aligned}
$$

The right side of Eqs. (3.24) and (3.26) of the forward and adjoint problems has a $v^{-1}$ factor important to formulating the transition rate matrix. In the forward problem, multiplying both sides of Eq. (3.24) by the velocity results in a speed $[\mathrm{cm} / \mathrm{s}]$ multiplied by a probability and a decay constant $\left[\mathrm{s}^{-1}\right]$ in the second term. This quantity does not correspond to a physical transition rate and it is unclear how this quantity may be tallied during a Monte Carlo simulation. However, in the adjoint formulation, multiplying both sides of Eq. (3.26) by the velocity results in a speed $[\mathrm{cm} / \mathrm{s}]$ multiplied by a cross section $\left[\mathrm{cm}^{-1}\right]$ in both the first and second terms. These quantities are rates $\left[\mathrm{s}^{-1}\right]$ describing the nature of neutron and precursor behavior within the system.

\section{Interpretation of the Adjoint Terms}

Close examination of each of these rates in the adjoint $\alpha$-eigenvalue equation shows their physical meaning. After multiplying the velocity term, the operators of the adjoint neutron transport equation become

$$
\begin{aligned}
& v \mathbf{M}^{\dagger}=-\underset{\mathbf{\Omega}}{1^{\dagger}} \nabla+v \Sigma(\mathbf{r}, E)-\iint v \Sigma_{s}\left(\mathbf{r} ; E, \hat{\mathbf{\Omega}} \rightarrow E^{\prime}, \hat{\mathbf{\Omega}}^{\prime}\right) d E^{\prime} d \mathbf{\Omega}^{\prime}, \\
& v \mathbf{F}_{\mathbf{p}}^{\dagger}=\iint(1-\beta) \bar{\nu}(E) v \Sigma_{f}(\mathbf{r}, E) \frac{\chi_{p}\left(E^{\prime}\right)}{4 \pi} d E^{\prime} d \mathbf{\Omega}^{\prime}, \\
& v \mathbf{F}_{\mathbf{d}_{j}^{\dagger}}^{\dagger}=\beta_{j} \bar{\nu}(E) v \Sigma_{f}(\mathbf{r}, E),
\end{aligned}
$$


where the speed is energy dependent

$$
v=\sqrt{\frac{2 E}{m}} .
$$

The adjoint precursor concentration equations have no extra speed term, and therefore the operators remain the same

$$
\iint \frac{\chi_{j}\left(E^{\prime}\right)}{4 \pi} \lambda_{j} d E^{\prime} d \Omega^{\prime} \text { and } \quad-\lambda_{j}
$$

The physical meanings of these seven mathematical expressions provides insight into the operator of the adjoint $\alpha$-eigenvalue problem and shows how it describes a TRM. Because these are operators, the integrated quantities are somewhat meaningless without an operand: in this case it is the adjoint angular flux. The shorthand $\mathbf{r}, E, \hat{\Omega}$ means "position $\mathbf{r}$, energy $E$, and direction $\hat{\Omega}$ ".

(1) ${ }^{\dagger}$ This is the reverse leakage rate for a neutron at $\mathbf{r}, E, \hat{\mathbf{\Omega}}$

$$
(1)^{\dagger}=-v \hat{\Omega} \cdot \nabla \text {. }
$$

Expanding the directional derivative in Cartesian coordinates yields

$$
\left(1^{\dagger}=-v\left(\Omega_{x} \frac{\partial}{\partial x}+\Omega_{y} \frac{\partial}{\partial y}+\Omega_{z} \frac{\partial}{\partial z}\right),\right.
$$

where $\Omega_{i}$ is the $i$-direction cosine. In the TRM interpretation, this is the transition rate of a energy $E$ neutron at $\mathbf{r}$ to $\mathbf{r}^{\prime}$ in direction $\hat{\Omega}$. By defining the $i$-direction speed as $v_{i}$, the equation simplifies to

$$
\text { (1) }^{\dagger}=-\left(v_{x} \frac{\partial}{\partial x}+v_{y} \frac{\partial}{\partial y}+v_{z} \frac{\partial}{\partial z}\right) .
$$

(2) ${ }^{\dagger}$ This is the collision rate of neutrons at $\mathbf{r}, E$ traveling in any direction

$$
(2)^{\dagger}=v \Sigma(\mathbf{r}, E)
$$

In the TRM interpretation, this is the transition rate of a neutron at $\mathbf{r}, E$ into another state via a collision. The collision type determines the state to which the neutron transfers. For example, separating the total cross section, $\Sigma(\mathbf{r}, E)$, 
into its scattering and absorption components yields

$$
(2)^{\dagger}=v \Sigma_{s}(\mathbf{r}, E)+v \Sigma_{a}(\mathbf{r}, E)
$$

and the term becomes the transition rate of a neutron at $\mathbf{r}, E, \hat{\Omega}$ to a neutron at $\mathbf{r}, E^{\prime}, \hat{\mathbf{\Omega}}^{\prime}$ plus the absorption rate of neutrons at $\mathbf{r}, E, \hat{\mathbf{\Omega}}$. This example extends to include fission or inelastic scattering.

(3) This is the total scattering rate of neutrons at $\mathbf{r}, E, \hat{\Omega}$ to all resulting energies and angles

$$
3^{\dagger}=\iint v \Sigma_{s}\left(\mathbf{r} ; E, \hat{\mathbf{\Omega}} \rightarrow E^{\prime}, \hat{\mathbf{\Omega}}^{\prime}\right) d E^{\prime} d \mathbf{\Omega}^{\prime} .
$$

This double integral shows the effect of using the adjoint scattering operator. The quantity within the integral is the scattering rate of neutrons at $\mathbf{r}, E, \hat{\mathbf{\Omega}}$ to $\mathbf{r}, E^{\prime}, \hat{\mathbf{\Omega}}^{\prime}$ : this interpretation is permissible because $v$ is dependent on $E$ and not $E^{\prime}$ as in the forward operator. It is integrated over all resulting energies and angles

$$
3^{\dagger}=\int_{4 \pi} d \mathbf{\Omega}^{\prime} \int_{0}^{\infty} d E^{\prime} v \Sigma_{s}\left(\mathbf{r} ; E, \hat{\mathbf{\Omega}} \rightarrow E^{\prime}, \hat{\mathbf{\Omega}}^{\prime}\right) .
$$

In the TRM interpretation, the quantity within the integral is the transition rate of a neutron at $\mathbf{r}, E, \hat{\boldsymbol{\Omega}}$ to $\mathbf{r}, E^{\prime}, \hat{\mathbf{\Omega}}^{\prime}$, while the integrated quantity is the transition rate of a neutron out of $\mathbf{r}, E, \hat{\Omega}$ via a scattering event.

(4) ${ }^{\dagger}$ This is the total prompt neutron emission rate from a fission induced by a neutron at $\mathbf{r}, E, \hat{\boldsymbol{\Omega}}$. The integrated quantity includes all prompt emission energies and angles

$$
\left(4^{\dagger}=\iint(1-\beta) \bar{\nu}(E) v \Sigma_{f}(\mathbf{r}, E) \frac{\chi_{p}\left(E^{\prime}\right)}{4 \pi} d E^{\prime} d \mathbf{\Omega}^{\prime}\right.
$$

where the emission angular distribution is isotropic. The combined first two terms within the integrand, $(1-\beta)$ and $\bar{\nu}(E)$, is the average number of prompt neutrons emitted for an induced fission at $E$. Together, the next two terms, $v \Sigma_{f}(\mathbf{r}, E)$, describe the rate of fission for a neutron at $\mathbf{r}, E, \hat{\mathbf{\Omega}}$ : in the forward formulation $v$ is dependent on the emission energy $E^{\prime}$. Finally, the $\chi_{p}\left(E^{\prime}\right)$ term determines the energy spectrum of the emitted prompt neutrons. In the TRM interpretation, these unintegrated terms are the transition rate of a neutron at $\mathbf{r}, E, \hat{\mathbf{\Omega}}$ to $(1-\beta) \bar{\nu}(E)$ neutrons at $\mathbf{r}, E^{\prime}, \hat{\mathbf{\Omega}}^{\prime}$. Prompt neutrons are emitted immediately after neutron absorption: the time for induced prompt neutron emission is negligible. Removing the terms dependent on $E$ from the integrand 
yields

$$
\text { (4) }^{\dagger}=(1-\beta) \bar{\nu}(E) v \Sigma_{f}(\mathbf{r}, E) \int_{4 \pi} d \boldsymbol{\Omega}^{\prime} \int_{0}^{\infty} d E^{\prime} \frac{\chi_{p}\left(E^{\prime}\right)}{4 \pi},
$$

where the total integrated quantity is the total transition rate of neutrons at $\mathbf{r}, E, \hat{\Omega}$ to $\bar{\nu}(E)$ neutrons at $\mathbf{r}$ in all energies and directions.

(5) ${ }^{\dagger}$ This is the total precursor emission rate into group $j$ from a fission induced by a neutron at $\mathbf{r}, E, \hat{\boldsymbol{\Omega}}$

$$
5^{\dagger}=\beta_{j} \bar{\nu}(E) v \Sigma_{f}(\mathbf{r}, E)
$$

The first term, $\beta_{j}$, is the probability of a group $j$ delayed neutron precursor emission, and the remaining terms are similar to those described for prompt neutron emission. In the TRM interpretation, this quantity is the transition rate of a neutron at $\mathbf{r}, E, \hat{\Omega}$ to $\beta_{j} \bar{\nu}(E)$ group $j$ precursors in at $\mathbf{r}$.

(6) This is the total emission rate of delayed neutrons from a precursor decaying at $\mathbf{r}$

$$
6^{\dagger}=\iint \frac{\chi_{j}\left(E^{\prime}\right)}{4 \pi} \lambda_{j} d E^{\prime} d \boldsymbol{\Omega}^{\prime}
$$

where the emission angular distribution is isotropic. In the TRM interpretation, the quantity within the integrand is the transition rate of a group $j$ precursor at $\mathbf{r}$ to a neutron at $\mathbf{r}, E^{\prime}, \hat{\mathbf{\Omega}}^{\prime}$. The integral is rewritten as

$$
\text { (6) }^{\dagger}=\lambda_{j} \int_{4 \pi} d E^{\prime} \int_{0}^{\infty} d \Omega^{\prime} \frac{\chi_{j}\left(E^{\prime}\right)}{4 \pi} .
$$

(7) This is the decay rate of group $j$ precursors at $\mathbf{r}$, which is equal to the decay constant

$$
(7)^{\dagger}=\lambda_{j},
$$

which is independent of position. In the TRM interpretation, this is the transition rate of a group $j$ precursor at position $\mathbf{r}$ to a neutron of position $\mathbf{r}$ in all energies and directions.

All of these terms describe physical quantities that support the interpretation of the adjoint operator as a TRM describing neutron transport. Furthermore, these quantities are obtainable during a Monte Carlo random walk: this is not the case with the operator of the forward $\alpha$-eigenvalue problem. 


\section{Matrix Formulation}

Replacing $\phi$ with $\psi(\mathbf{r}, E, \hat{\mathbf{\Omega}})$ in Eq. (2.52) gives the matrix form of the forward $\alpha$ eigenvalue problem

$$
\left[\begin{array}{ll}
v & 0 \\
0 & I
\end{array}\right]\left[\begin{array}{cc}
-\mathbf{M}+\mathbf{F}_{\mathbf{p}} & \chi \boldsymbol{\lambda} \\
\mathbf{F}_{\mathbf{d}} & -\boldsymbol{\lambda}
\end{array}\right]\left[\begin{array}{l}
\psi \\
\boldsymbol{C}
\end{array}\right]=\alpha\left[\begin{array}{c}
\psi(\mathbf{r}, E, \hat{\mathbf{\Omega}}) \\
\boldsymbol{C}(\mathbf{r})
\end{array}\right]
$$

rewritten here for ease of reference. Similarly, the adjoint problem is

$$
\left[\begin{array}{ll}
v & 0 \\
0 & I
\end{array}\right]\left[\begin{array}{cc}
-\mathbf{M}^{\dagger}+\mathbf{F}_{\mathbf{p}}^{\dagger} & \mathbf{F}_{\mathbf{d}}^{\dagger} \\
(\boldsymbol{\chi} \boldsymbol{\lambda})^{\dagger} & -\boldsymbol{\lambda}
\end{array}\right]\left[\begin{array}{l}
\psi^{\dagger} \\
\boldsymbol{C}^{\dagger}
\end{array}\right]=\alpha^{\dagger}\left[\begin{array}{c}
\psi^{\dagger}(\mathbf{r}, E, \hat{\boldsymbol{\Omega}}) \\
\boldsymbol{C}^{\dagger}(\mathbf{r})
\end{array}\right]
$$

where $\boldsymbol{C}^{\dagger}$ is a length $J$ column vector of the adjoint precursor group concentrations $C_{j}^{\dagger}, \mathbf{F}_{\mathbf{d}}^{\dagger}$ is a length $J$ row vector of adjoint group delayed fission operators $\mathbf{F}_{\mathbf{d} j}^{\dagger},(\boldsymbol{\chi} \boldsymbol{\lambda})^{\dagger}$ is a length $J$ column vector of the adjoint precursor emission operators $\left(\chi_{j} \lambda_{j}\right)^{\dagger}$, and $\boldsymbol{\lambda}$ is the same size $J \times J$ diagonal matrix with decay constants $\lambda_{j}$ on the diagonal seen in the forward formulation. In this formulation, transposing the matrix of operators in Eq. (3.46) yields the matrix in the adjoint $\alpha$-eigenvalue problem in Eq. (3.47), and vice versa, where the eigenvalue sets $\alpha$ and $\alpha^{\dagger}$ are complex conjugates. This is not readily apparent due to the differences in the forward and adjoint delayed fission operators, but it is shown in the next section. Note that the speed matrix is not transposed in Eq. (3.47) due to the $\alpha$ eigenvalue remaining associated with the neutron speeds in both the forward and adjoint formulations.

The TRMM is able to obtain the product of the speed and adjoint matrices on the left side of Eq. (3.47) due to its natural interpretation as a matrix of transition rates describing neutrons and precursors moving about a system. This is the TRM

$$
\mathbf{Q}=\left[\begin{array}{cc}
v\left(-\mathbf{M}+\mathbf{F}_{\mathbf{p}}\right)^{\dagger} & v \mathbf{F}_{\mathbf{d}}^{\dagger} \\
(\boldsymbol{\chi} \boldsymbol{\lambda})^{\dagger} & -\boldsymbol{\lambda}
\end{array}\right]
$$

With this matrix, the TRMM solves the simple eigenvalue problem for the adjoint eigenpairs, $\alpha_{i}^{\dagger}$ and $\left[\psi_{i}^{\dagger} \boldsymbol{C}_{i}^{\dagger}\right]$. But, the adjoint eigenpairs are not particularly useful without the solutions to the forward problems. Therefore, the TRMM also obtains the speed matrix to calculate the forward problem and solve for the forward eigenpairs, $\alpha_{i}$ and $\left[\psi_{i} \boldsymbol{C}_{i}\right]$, providing the whole solution to the problem, and a direct way of solving for the $\alpha$-eigenvalue spectrum and eigenfunctions. 


\subsubsection{The Forward and Adjoint $\alpha$-Eigenvalue Problems}

The relation between the operator matrices in the forward and adjoint $\alpha$-eigenvalue problems is

$$
\left[\begin{array}{cc}
-\mathbf{M}+\mathbf{F}_{\mathbf{p}} & \chi \boldsymbol{\lambda} \\
\mathbf{F}_{\mathbf{d}} & -\boldsymbol{\lambda}
\end{array}\right]=\left[\begin{array}{cc}
-\mathbf{M}^{\dagger}+\mathbf{F}_{\mathbf{p}}^{\dagger} & \mathbf{F}_{\mathbf{d}}^{\dagger} \\
(\chi \boldsymbol{\lambda})^{\dagger} & -\boldsymbol{\lambda}
\end{array}\right]^{\mathbf{T}}
$$

because the matrix is all-real. There are four sections of these matrices: each follows this relation. Starting with the top left in the forward operator matrix and proceeding clockwise, the sections are prompt neutron effects, precursor emission, precursor decay, and delayed fission. Examining these individually reveals the property in Eq. (3.49).

\section{Transposing the Operator Matrix}

The precursor decay section is the simplest, as it remains unchanged in the forward and adjoint matrices because it is square diagonal:

$$
\left[\begin{array}{ccc}
-\lambda_{1} & & \\
& \ddots & \\
& & -\lambda_{J}
\end{array}\right]
$$

The entries operate on precursor concentrations:

$$
-\lambda_{j} C_{j}(\mathbf{r}) \Rightarrow-\lambda_{j} C_{j}^{\dagger}(\mathbf{r})
$$

Including moving fuel destroys this diagonal structure, and requires an additional term in the precursor equation.

The precursor emission section is rectangular and operates on functions with a different phase space in the forward and adjoint operator matrices:

$$
\chi_{j}(E) \lambda_{j} C_{j}(\mathbf{r}) \Rightarrow \iint \frac{\chi_{j}\left(E^{\prime}\right)}{4 \pi} \lambda_{j} \psi^{\dagger}\left(\mathbf{r}, E^{\prime}, \hat{\mathbf{\Omega}}^{\prime}\right) d E^{\prime} d \mathbf{\Omega}^{\prime} .
$$

In the forward operator, entries distributed in the position-energy-direction phase space operate on the precursor concentrations. In the adjoint operator, entries distributed in the position phase space operate on the adjoint angular flux. This change represents transposing the phase space. The double integral in the adjoint operator is necessary to change from operating on precursor concentrations to operating on adjoint angular flux: integrals in the adjoint formulation yield the same entries as in 
the forward formulation.

Similar to the precursor decay section, the delayed fission section is rectangular:

$$
\iint \beta_{j} \bar{\nu}\left(E^{\prime}\right) \Sigma_{f}\left(\mathbf{r}, E^{\prime}\right) \psi\left(\mathbf{r}, E^{\prime}, \hat{\mathbf{\Omega}}^{\prime}\right) d E^{\prime} d \mathbf{\Omega}^{\prime} \Rightarrow \beta_{j} \bar{\nu}(E) \Sigma_{f}(\mathbf{r}, E) C_{j}^{\dagger}(\mathbf{r}) .
$$

In the forward operator, entries distributed in the position phase space operate on the angular neutron flux. In the adjoint operator, entries distributed in the positionenergy-direction phase space operate on the adjoint precursor concentration. The adjoint operator lacks an integral because they operate on the adjoint precursor concentrations instead of the angular neutron flux.

The prompt neutron effects section has numerous parts due to the complexities of the $\mathbf{M}$ and $\mathbf{F}_{\mathbf{p}}$ operators. This section is a square matrix because the phase spaces of the angular and adjoint angular fluxes are the same. In the forward operator, the entries distributed in the position-energy-direction phase space operate on the angular neutron flux: in the adjoint operator, they operate on the adjoint angular flux. The leakage term changes in sign:

$$
\hat{\boldsymbol{\Omega}} \cdot \nabla \psi(\mathbf{r}, E, \hat{\mathbf{\Omega}}) \Rightarrow-\hat{\mathbf{\Omega}} \cdot \nabla \psi^{\dagger}(\mathbf{r}, E, \hat{\mathbf{\Omega}}),
$$

where the negative implies that the entries distributed in the position-energy-direction phase space operate on the neutron importance at the final location instead of the neutron flux at the current location as in the forward operator. This change represents transposing the entry in the matrix. The collision term is the same in the forward and adjoint operators:

$$
\Sigma(\mathbf{r}, E) \psi(\mathbf{r}, E, \hat{\boldsymbol{\Omega}}) \Rightarrow \Sigma(\mathbf{r}, E) \psi^{\dagger}(\mathbf{r}, E, \hat{\boldsymbol{\Omega}}) .
$$

In either case, entries distributed in the position-energy-direction phase space operate on the same space: these entries are on the diagonal and are stationary during the transpose. The variables of integration in the scattering term change:

$$
\iint \Sigma_{s}\left(\mathbf{r} ; E^{\prime}, \hat{\boldsymbol{\Omega}}^{\prime} \rightarrow E, \hat{\boldsymbol{\Omega}}\right) \psi^{\prime} d E^{\prime} d \boldsymbol{\Omega}^{\prime} \Rightarrow \iint \Sigma_{s}\left(\mathbf{r} ; E, \hat{\boldsymbol{\Omega}} \rightarrow E^{\prime}, \hat{\mathbf{\Omega}}^{\prime}\right) \psi^{\dagger \prime} d E^{\prime} d \boldsymbol{\Omega}^{\prime} .
$$

The integrals in the forward and adjoint operators are over the incoming and outgoing neutron energy and direction, respectively. In the forward operator, entries distributed in the position-energy-direction phase space operate on the incoming neutron flux. In the adjoint operator, entries distributed in the position-energy-direction 
phase space operate on the outgoing neutron importance. This is similar to the leakage term, and is equivalent to transposing the entry. The same reasoning applies to the change in the prompt fission operator:

$$
\begin{aligned}
\iint \frac{\chi_{p}(E)}{4 \pi}(1-\beta) \bar{\nu}\left(E^{\prime}\right) \Sigma_{f}\left(\mathbf{r}, E^{\prime}\right) \psi^{\prime} d E^{\prime} d \mathbf{\Omega}^{\prime} \Rightarrow \\
\iint(1-\beta) \bar{\nu}(E) \Sigma_{f}(\mathbf{r}, E) \frac{\chi_{p}\left(E^{\prime}\right)}{4 \pi} \psi^{\dagger \prime} d E^{\prime} d \mathbf{\Omega}^{\prime}
\end{aligned}
$$

\section{The Adjoint Spectrum}

Properties of the forward $\alpha$-eigenvalue spectrum are discussed in $\S 2.3 .3$. There it is noted that because the forward operator $\mathbf{A}$ is all-real, its complex eigenvalues come in conjugate pairs. This is also true with the adjoint operator $\mathbf{Q}$. Furthermore, the forward and adjoint $\alpha$-eigenvalue spectra are complex conjugates of one another: in a plot of these eigenvalues in the complex plane, they align and fall on top of one another. Thus, it is difficult to distinguish between identical and complex conjugate spectra when the adjoint and forward $\alpha$ eigenvalue spectra contain the same eigenvalues. To show this property of the adjoint and forward spectra, consider the adjoint problem in terms of the tallied TRM

$$
\mathbf{Q} u^{\dagger}=\alpha u^{\dagger}
$$

where $^{\dagger}$ is removed from the eigenvalue and the eigenfunctions are denoted by $u=$ $\vec{u}=\left[\psi C_{j}\right]$ for simplification. Operating by the inverse speed matrix on both sides, multiplying by the conjugate forward eigenfunction $\bar{u}$, and revolving the functions of the scalar products yields the forward problem in Eq. (3.46) in terms of the TRM,

$$
\mathbf{V Q}^{\mathbf{T}} \mathbf{V}^{-1} u=\alpha u
$$

where the eigenvalue is the same as in the adjoint problem and the speed matrix is

$$
\mathbf{V}=\left[\begin{array}{ll}
v & 0 \\
0 & I
\end{array}\right]
$$

Thus, the eigenvalue spectra for the forward and adjoint $\alpha$-eigenvalue problem contain the same sets of eigenvalues, and this same formulation is applicable for a discrete 
problem. This is also shown by comparing the determinants of the matrices

$$
\operatorname{det}[\mathbf{Q}-\lambda I]=\operatorname{det}\left[\mathbf{V Q}^{\mathbf{T}} \mathbf{V}^{-1}-\lambda I\right] .
$$

Equality is shown with some algebra and a few matrix properties: (i) apply the property $\operatorname{det}[A]=\operatorname{det}\left[A^{\mathbf{T}}\right]$, (ii) separate out the speed matrices, (iii) note that $\mathbf{V} I \mathbf{V}^{-1}=I$, (iv) apply the property $\operatorname{det}[A B]=\operatorname{det}[A] \operatorname{det}[B]$, and (v) apply the property $\operatorname{det}\left[A^{-1}\right]=\operatorname{det}[A]^{-1}$.

The forward and adjoint eigenfunctions, $u$ and $u^{\dagger}$, are entirely different. This is no surprise when considering that the forward and adjoint equations describe very different physical mechanisms. With some matrix algebra, the forward and adjoint eigenfunctions are recast as left eigenfunctions

$$
\begin{aligned}
u \mathbf{V}^{-1} \mathbf{Q V} & =\alpha u, \\
u^{\dagger} \mathbf{Q}^{\mathbf{T}} & =\alpha^{\dagger} u^{\dagger},
\end{aligned}
$$

of the transposed matrices. While this is potentially useful because it avoids transposing the TRM, the TRMM does not use these formulas during calculations.

The bi-orthogonality condition of the forward and adjoint $\alpha$ eigenfunctions is important to the derivation of the time expansion functions for the solution to the time-dependent flux. Working the forward and adjoint $\alpha$-eigenvalue problems into similar form an subtracting the two results gives the bi-orthogonality condition of the forward and adjoint $\alpha$ eigenfunctions

$$
0=\left(\alpha_{i}-\alpha_{j}^{\dagger}\right)\left(u_{j}^{\dagger} \mathbf{V}^{-1} u_{i}\right) .
$$

It follows that, in the case when $\alpha_{i} \neq \alpha_{j}^{\dagger}$,

$$
\left(u_{j}^{\dagger} \mathbf{V}^{-1} u_{i}\right)=0 .
$$

Conversely, if this quantity is not zero, then $\alpha_{i}=\alpha_{j}^{\dagger}$.

\subsubsection{Corollary to the Fission Matrix}

The TRM is the continuous-time analog of the fission matrix $\mathbf{F}$ for multiplying media. For the fission matrix, the geometry is divided into a set of regions. Then, an element of the fission matrix $f_{i, j}$ is next-generation fission neutrons produced in region $i$ for each average fission neutron starting in region $j$. While the TRM has unnormal- 
ized rates describing neutrons moving among a discretized position-energy-direction phase space, the fission matrix has normalized probabilities describing induced fissions throughout the discretized position phase space [5]. The eigenpairs of the fission matrix are estimates of the $k$ eigenvalues and eigenfunctions, while the accuracy of the estimate increases with larger numbers of regions. These eigenpairs have several applications, including to source expansions and convergence acceleration.

In the context of Markov processes, the state space of the TRM and fission matrix are both discretized. The TRM has a continuous-time parameter space, while the fission matrix has a discrete-time parameter space. For the fission matrix, neutrons are tracked between fission sites, so the discrete-time parameter space are fission generations $T=\left\{t_{0}, t_{1}, t_{2}, \cdots\right\}$. Thus, the fission matrix describes a discrete-time Markov chain, as the TRM describes a continuous-time Markov chain.

Additionally, the TRMM and the Monte Carlo method for tallying the fission matrix also share similar convergence behavior due to the generation of probabilities and rates via stochastic methods [6]. Both matrices are considerably sparse, while the TRM is considerably larger due to the additional energy-direction phase space. Sparse eigenvalue solvers are important for both methods. Due to the simplicity of the $k$-eigenvalue spectrum, e.g., it has no complex eigenvalues, some solvers tend to work better and more efficiently for finding the eigenvalues of the fission matrix. As supported by theoretical arguments and empirical evidence [5], the eigenvalues of the fission matrix converge to the real $k$ eigenvalues of the system as the number of spatial regions increases, in the order of the most dominant to the least dominant. The TRMM follows similar convergence behavior.

\subsection{Derivations of Transition Rate Matrices}

The TRM as shown in Eq. (3.48) describes a matrix of arbitrary size. In terms of Markov processes, this means that the state space is continuous, where in the case of neutron transport, this is the position-energy-direction phase space. But, to compute eigenpairs of a matrix, it must be finite, where a full-rank matrix of size $n \times n$ yields $n$ eigenpairs. Thus, the size of the TRM is made finite by defining the states of the Markov process as a neutron meeting certain conditions in the phase space $\mathbf{r}_{i}, E_{b}, \hat{\Omega}_{k} \in \mathbf{r}, E, \hat{\Omega}$. In doing this, the continuous state space reduces to a discrete state space, and a Markov chain approximates the behavior of a Markov process. If the definition of the states of the Markov process is fine enough, or the TRM is large enough, this solution is an adequate approximation of the true continuous solution. 
The same is said for the calculated $\alpha$-eigenvalue spectrum: in limiting the state space, the TRMM calculates a finite number of $\alpha$ eigenvalues that serve as an approximation to the full spectrum. The larger the TRM, the better this approximation is to the full spectrum.

This section shows derivations of the TRM starting from the adjoint equations without approximations: the weighting function in these formulations is adjoint importance. The following section discusses the estimation of the TRM elements using forward-weighted quantities during the Monte Carlo random walk.

Up to this point, formulations provided are general forms of the forward and adjoint $\alpha$-eigenvalue problems considering space and direction. Applications of the TRMM to problems with simplified geometries show the methodology behind defining the state space and the nature of the Markov TRM in Eq. (3.1). Use of simplistic media also allows for studying the $\alpha$-eigenvalue spectrum, convergence behavior, and the effect of state definition, among other things. Infinite media are the simplest of these problems.

\subsubsection{Infinite Medium}

Infinite medium problems are position and direction independent: the state space includes only energy. Define $G+J$ states of the Markov process, where $G$ states define neutrons in energy space and $J$ states define delayed neutron precursor groups. Specifically, if a particle is a neutron of energy $E$ meeting the condition $E_{g+1}<E<$ $E_{g}$, the Markov process is in state $g$. This is akin to discretizing the energy space. Likewise, if a particle is a delayed neutron precursor in group $j$, then the Markov process is in state $G+j$. This results in a TRM of size $(G+J) \times(G+J)$, with $G+J$ eigenpairs. The number of non-zero entries of this matrix is often much less than $(G+J)^{2}$, especially for large $G$. This is not very memory intensive even if considering a hyperfine definition of the energy states, such as $G=2000$. For cases with ${ }^{235} \mathrm{U}$, $J=6$ to reflect the number of precursor groups available in the ENDF database $[7,8]$.

The following is a rigorous derivation of the TRM for the continuous-energy prob- 
lem. The adjoint equation for an infinite medium is

$$
\begin{aligned}
-\frac{1}{v} \frac{\partial \psi^{\dagger}}{\partial t}+\Sigma \psi^{\dagger}(E, t)= & \int \Sigma_{s}\left(E \rightarrow E^{\prime}\right) \psi^{\dagger}\left(E^{\prime}, t\right) d E^{\prime} \\
& +\int(1-\beta) \bar{\nu} \Sigma_{f} \chi_{p}\left(E^{\prime}\right) \psi^{\dagger}\left(E^{\prime}, t\right) d E^{\prime}+\sum_{j=1}^{J} \beta_{j} \bar{\nu} \Sigma_{f} C_{j}^{\dagger} \\
-\frac{\partial C_{j}^{\dagger}}{\partial t}+\lambda_{j} C_{j}^{\dagger}(t)= & \int \chi_{j}\left(E^{\prime}\right) \lambda_{j} \psi^{\dagger}\left(E^{\prime}, t\right) d E^{\prime}
\end{aligned}
$$

Introducing the $\alpha$-eigenvalue, multiplying by the velocity, and rearranging terms yields the adjoint $\alpha$-eigenvalue problem for an infinite medium

$$
\begin{gathered}
-v \Sigma \psi^{\dagger}(E)+\int v \Sigma_{s}\left(E \rightarrow E^{\prime}\right) \psi^{\dagger}\left(E^{\prime}\right) d E^{\prime}+\int(1-\beta) \bar{\nu} v \Sigma_{f} \chi_{p}\left(E^{\prime}\right) \psi^{\dagger}\left(E^{\prime}\right) d E^{\prime} \\
+\sum_{j=1}^{J} \beta_{j} \bar{\nu} v \Sigma_{f} C_{j}^{\dagger}=\alpha \psi^{\dagger} \\
-\lambda_{j} C_{j}^{\dagger}+\int \chi_{j}\left(E^{\prime}\right) \lambda_{j} \psi^{\dagger}\left(E^{\prime}\right) d E^{\prime}=\alpha C_{j}^{\dagger}
\end{gathered}
$$

Now, discretize the energy space into $G$ energy intervals between $E_{G+1}$ and $E_{1}$ where the $g$ th energy interval is defined as $E_{g+1}<E<E_{g}$. Integrate the neutron importance over the $g$ th energy interval, defining the importance in interval $g$ as

$$
\psi_{g}^{\dagger}=\int_{E_{g+1}}^{E_{g}} d E \psi^{\dagger}(E)
$$

and $\vec{\psi}^{\dagger}$ as the vector containing these importances

$$
\vec{\psi}^{\dagger}=\psi_{1}^{\dagger} \ldots \psi_{G}^{\dagger}
$$

Integrate Eq. (3.68) over the $g$ th energy interval and multiply and divide by $\psi_{g}^{\dagger}$. The collision term becomes

$$
\int_{E_{g+1}}^{E_{g}} d E v \Sigma(E) \psi^{\dagger}(E)=\left[\left(v \Sigma_{t}\right)_{g}^{\dagger}\right] \psi_{g}^{\dagger}
$$

where

$$
\left(v \Sigma_{t}\right)_{g}^{\dagger}=\frac{1}{\psi_{g}^{\dagger}} \int_{E_{g+1}}^{E_{g}} d E v \Sigma(E) \psi^{\dagger}(E) .
$$

The integral over all energies in the scattering term must be rewritten in the terms 
of a sum

$$
\begin{aligned}
\int_{E_{g+1}}^{E_{g}} v d E \int_{0}^{\infty} d E^{\prime} \Sigma_{s}(E & \left.\rightarrow E^{\prime}\right) \psi^{\dagger}\left(E^{\prime}\right) \\
= & \sum_{g^{\prime}=1}^{G} \int_{E_{g+1}}^{E_{g}} v d E \int_{E_{g^{\prime}+1}}^{E_{g^{\prime}}} d E^{\prime} \Sigma_{s}\left(E \rightarrow E^{\prime}\right) \psi^{\dagger}\left(E^{\prime}\right),
\end{aligned}
$$

and the interval-transfer rate from interval $g \rightarrow g^{\prime}$ is defined as

$$
\left(v_{g} \Sigma_{s g \rightarrow g^{\prime}}\right)^{\dagger}=\frac{1}{\psi_{g^{\prime}}^{\dagger}} \int_{E_{g+1}}^{E_{g}} v d E \int_{E_{g^{\prime}+1}}^{E_{g^{\prime}}} d E^{\prime} \Sigma_{s}\left(E \rightarrow E^{\prime}\right) \psi^{\dagger}\left(E^{\prime}\right)
$$

The fission term is rewritten similarly as

$$
\begin{aligned}
\int_{E_{g+1}}^{E_{g}} d E(1-\beta) \bar{\nu}(E) v \Sigma_{f}(E) \int_{0}^{\infty} d E^{\prime} \chi_{p}\left(E^{\prime}\right) \psi^{\dagger}\left(E^{\prime}\right) \\
=\sum_{g^{\prime}=1}^{G} \int_{E_{g+1}}^{E_{g}} d E(1-\beta) \bar{\nu}(E) v \Sigma_{f}(E) \int_{E_{g^{\prime}+1}}^{E_{g^{\prime}}} d E^{\prime} \chi_{p}\left(E^{\prime}\right) \psi^{\dagger}\left(E^{\prime}\right) .
\end{aligned}
$$

The number of prompt neutrons emitted from interval $g$ multiplied by the fission rate in interval $g$ is defined as

$$
\left(\bar{\nu}_{p} v \Sigma_{f}\right)_{g}^{\dagger}=\int_{E_{g+1}}^{E_{g}} d E(1-\beta) \bar{\nu}(E) v \Sigma_{f}(E)
$$

and the fission-emission probability for interval $g^{\prime}$ is defined as

$$
\chi_{p g^{\prime}}^{\dagger}=\frac{1}{\psi_{g^{\prime}}^{\dagger}} \int_{E_{g^{\prime}+1}}^{E_{g^{\prime}}} d E^{\prime} \chi_{p}\left(E^{\prime}\right) \psi^{\dagger}\left(E^{\prime}\right)
$$

The $j$ th precursor term becomes

$$
\int_{E_{g+1}}^{E_{g}} d E \beta_{j} \bar{\nu}(E) v \Sigma_{f}(E) C_{j}^{\dagger}=\beta_{j}\left(\bar{\nu}_{d} v \Sigma_{f}\right)_{g}^{\dagger} C_{j}^{\dagger}
$$

where the adjoint precursor concentration is independent of energy. Define the inverse speed in interval $g$ as

$$
\frac{1}{v_{g}^{\dagger}}=\frac{1}{\psi_{g}^{\dagger}} \int_{E_{g+1}}^{E_{g}} d E \frac{1}{v} \psi^{\dagger}(E)
$$


Now, define the integral in Eq. (3.69) as a sum

$$
\int_{0}^{\infty} \chi_{j}\left(E^{\prime}\right) \lambda_{j} \psi^{\dagger}\left(E^{\prime}\right) d E^{\prime}=\sum_{g=1}^{G} \int_{E_{g+1}}^{E_{g}} d E^{\prime} \chi_{j}\left(E^{\prime}\right) \lambda_{j} \psi^{\dagger}\left(E^{\prime}\right)
$$

and define the $j$ th delayed precursor emission probability for interval $g$ as

$$
\chi_{j g}^{\dagger}=\frac{1}{\psi_{g}^{\dagger}} \int_{E_{g+1}}^{E_{g}} d E^{\prime} \chi_{j}\left(E^{\prime}\right) \psi^{\dagger}\left(E^{\prime}\right)
$$

With these definitions, the infinite medium adjoint $\alpha$-eigenvalue problem is written in a discretized form as

$$
\begin{aligned}
&-\left(v \Sigma_{t}\right)_{g}^{\dagger} \psi_{g}^{\dagger}+\sum_{g^{\prime}=1}^{G}\left(v_{g} \Sigma_{s g \rightarrow g^{\prime}}\right)^{\dagger} \psi_{g^{\prime}}^{\dagger}+\sum_{g^{\prime}=1}^{G}\left(\bar{\nu}_{p} v \Sigma_{f}\right)_{g}^{\dagger} \chi_{p g^{\prime}}^{\dagger} \psi_{g^{\prime}}^{\dagger} \\
&+\sum_{j=1}^{J} \beta_{j}\left(\bar{\nu}_{d} v \Sigma_{f}\right)_{g}^{\dagger} C_{j}^{\dagger}=\alpha \psi_{g}^{\dagger}, \\
&-\lambda_{j} C_{j}^{\dagger}+\sum_{g^{\prime}=1}^{G} \chi_{j g^{\prime}}^{\dagger} \lambda_{j} \psi_{g^{\prime}}^{\dagger}=\alpha C_{j}^{\dagger},
\end{aligned}
$$

To set the equations up in matrix form, rearrange terms and make some definitions. Define the removal rate to exclude the self scattering rate

$$
\left(v \Sigma_{r}\right)_{g}^{\dagger}=\left(v \Sigma_{t}\right)_{g}^{\dagger}-\left(v_{g} \Sigma_{s g \rightarrow g}\right)^{\dagger}
$$

and insert it into the equations, yielding

$$
\begin{aligned}
{\left[-\left(v \Sigma_{r}\right)_{g}^{\dagger}+\chi_{p g}^{\dagger}\left(\bar{\nu}_{p} v \Sigma_{f}\right)_{g}^{\dagger}\right] \psi_{g}^{\dagger}+\sum_{g^{\prime} \neq g}^{G}\left[\left(v_{g} \Sigma_{s g \rightarrow g^{\prime}}\right)^{\dagger}+\chi_{p g^{\prime}}^{\dagger}\left(\bar{\nu}_{p} v \Sigma_{f}\right)_{g}^{\dagger}\right] \psi_{g^{\prime}}^{\dagger} } & \\
& +\sum_{j=1}^{J} \beta_{j}\left(\bar{\nu}_{d} v \Sigma_{f}\right)_{g}^{\dagger} C_{j}^{\dagger}=\alpha \psi_{g}^{\dagger}, \\
& \sum_{g^{\prime}=1}^{G} \chi_{j g^{\prime}}^{\dagger} \lambda_{j} \psi_{g^{\prime}}^{\dagger}-\lambda_{j} C_{j}^{\dagger}=\alpha C_{j}^{\dagger} .
\end{aligned}
$$

The result is a matrix problem with four partitions describing particles (neutrons or precursors) moving within and between energy intervals and delayed neutron pre- 
cursor decay groups

$$
\mathbf{Q}=\left[\begin{array}{ll}
\mathbf{Q}_{g \rightarrow g^{\prime}} & \mathbf{Q}_{g \rightarrow j} \\
\mathbf{Q}_{j \rightarrow g} & \mathbf{Q}_{j \rightarrow j^{\prime}}
\end{array}\right]
$$

where $\mathbf{Q}_{g \rightarrow g^{\prime}}$ describes the transition of neutrons between energy intervals, $\mathbf{Q}_{g \rightarrow j}$ pertains to neutrons inducing fission resulting in the production of delayed neutron precursors, $\mathbf{Q}_{j \rightarrow g}$ represents the emission of neutrons from precursors, and $\mathbf{Q}_{j \rightarrow j^{\prime}}$ represents the decay (removal) of the precursors. These sections relate directly to the Q matrix in Eq. (3.48), where $\mathbf{Q}_{g \rightarrow g^{\prime}}$ is

$$
v\left(-\mathbf{M}+\mathbf{F}_{\mathbf{p}}\right)^{\dagger}=\left[\begin{array}{ccc}
-\left(v \Sigma_{r}\right)_{1}^{\dagger}+\chi_{p 1}^{\dagger}\left(\bar{\nu}_{p} v \Sigma_{f}\right)_{1}^{\dagger} & \left(v_{1} \Sigma_{s 12}\right)^{\dagger}+\chi_{p 2}^{\dagger}\left(\bar{\nu}_{p} v \Sigma_{f}\right)_{1}^{\dagger} & \cdots \\
\left(v_{2} \Sigma_{s 21}\right)^{\dagger}+\chi_{p 1}^{\dagger}\left(\bar{\nu}_{p} v \Sigma_{f}\right)_{2}^{\dagger} & -\left(v \Sigma_{r}\right)_{2}^{\dagger}+\chi_{p 2}^{\dagger}\left(\bar{\nu}_{p} v \Sigma_{f}\right)_{2}^{\dagger} & \cdots \\
\vdots & \vdots & \ddots
\end{array}\right],
$$

where $\Sigma_{s i j}=\Sigma_{s i \rightarrow j}$. This has two distinct parts: the diagonal and off-diagonal elements. The diagonal element of row $g$ of the matrix is

$$
\begin{array}{r}
-\left(v \Sigma_{r}\right)_{g}^{\dagger}+\chi_{p g}^{\dagger}\left(\bar{\nu}_{p} v \Sigma_{f}\right)_{g}^{\dagger}=-(\text { removal rate from energy interval } g) \\
+(\text { fission-emission rate from energy interval } g \rightarrow g),
\end{array}
$$

which is the negative net removal rate of neutrons from energy interval $g$. The fission-emission rate is defined as the fission rate multiplied by the average number of neutrons released per fission. In the diagonal elements, this rate is also known as the self fission-emission rate because the fission event occurs and releases a neutron in the same energy interval. The off-diagonal element of row $g$ and column $g^{\prime} \neq g$ of the matrix is

$$
\begin{array}{r}
\left(v_{g} \Sigma_{s g g^{\prime}}\right)^{\dagger}+\chi_{p g^{\prime}}^{\dagger}\left(\bar{\nu}_{p} v \Sigma_{f}\right)_{g}^{\dagger}=(\text { scatter rate from energy interval } g \\
+(\text { fission-emission rate from energy interval } g
\end{array}
$$

which is the net transition rate from energy interval $g \rightarrow g^{\prime}$. In the context of the TRM interpretation described in Eq. (3.1), $q_{g g}$ is the transition rate out of energy interval $g$ and $q_{g g^{\prime}}$ is the transition rate from energy interval $g$ to $g^{\prime}$. In an infinite medium, a neutron leaves an energy interval due to collision, and reenters due to self fission and self scatter, as shown in Eq. (3.90). A neutron transitions between energy intervals by scattering or fission, the rates for which are shown in Eq. (3.91). The top 
right section $\mathbf{Q}_{g \rightarrow j}$ is

$$
v \mathbf{F}_{\mathbf{d}}^{\dagger}=\left[\begin{array}{lll}
v \mathbf{F}_{\mathbf{d} 1}^{\dagger} & \cdots & v \mathbf{F}_{\mathbf{d} J}^{\dagger}
\end{array}\right]=\left[\begin{array}{ccc}
\beta_{1}\left(\bar{\nu}_{d} v \Sigma_{f}\right)_{1}^{\dagger} & \cdots & \beta_{J}\left(\bar{\nu}_{d} v \Sigma_{f}\right)_{1}^{\dagger} \\
\vdots & \ddots & \vdots \\
\beta_{1}\left(\bar{\nu}_{d} v \Sigma_{f}\right)_{G}^{\dagger} & \cdots & \beta_{J}\left(\bar{\nu}_{d} v \Sigma_{f}\right)_{G}^{\dagger}
\end{array}\right]
$$

where the entries represent precursor-emission rates. The bottom left section $\mathbf{Q}_{j \rightarrow g}$ is

$$
(\boldsymbol{\chi} \boldsymbol{\lambda})^{\dagger}=\left[\begin{array}{c}
\left(\chi_{1} \lambda_{1}\right)^{\dagger} \\
\vdots \\
\left(\chi_{J} \lambda_{J}\right)^{\dagger}
\end{array}\right]=\left[\begin{array}{ccc}
\chi_{11}^{\dagger} \lambda_{1} & \cdots & \chi_{1 G}^{\dagger} \lambda_{1} \\
\vdots & \ddots & \vdots \\
\chi_{J 1}^{\dagger} \lambda_{J} & \cdots & \chi_{J G}^{\dagger} \lambda_{J}
\end{array}\right]
$$

where the entries represent delayed neutron emission rates. The bottom right partition is simply the diagonal matrix of decay constants $-\lambda_{j}$ in Eq. (3.50). Extending the TRM interpretation, $q_{g, G+j}$ is the transition rate from energy interval $g$ to precursor group $j$. A neutron transitions to a precursor group by delayed fission, the rates for which are shown in Eq. (3.92). This interpretation applies to the precursor section at the bottom of the TRM, where $q_{G+j, G+j}$ is the transition rate out of precursor group $j$, and $q_{G+j, g^{\prime}}$ is the transition rate from precursor group $j$ to energy interval $g^{\prime}$. A precursor from group $j$ decays at a rate of $\lambda_{j}$ and transitions to energy interval $g^{\prime}$ according to the delayed neutron emission spectrum, $\chi_{j g^{\prime}}$. These rates are shown in Eqs. (3.93) and (3.50). Upon absorption, neutrons transition to an absorbing state that is undefined within the matrix formulation. Because of this, the diagonal elements do not follow the requirement in Eq. (3.3).

\subsubsection{One-Dimensional Media}

For one-dimension, or slab geometry, the TRM formulation extends to include position and direction as well as energy. Treatment of the energy space is similar to that in the infinite medium case. Define $N G M+N J$ states of the Markov process, where $N G M$ states define neutrons distributed in the position-energy-direction phase space and $N J$ states define precursors distributed in the position-group space. A neutron with direction $\mu$ meeting the condition $\mu_{m-1}<\mu<\mu_{m}$ is said to be in direction interval $m$. Likewise, a neutron or precursor with position $x$ meeting the condition $x_{n-1}<x<x_{n}$ is said to be in position interval $n$. Together with the precursor groups and energy intervals, state $i$ of the Markov process is defined as a neutron within intervals $m, n$, and $g$ or a group $j$ precursor within interval $n$. The simplest one-dimensional problems are one-speed, with only one energy interval. 


\section{One-Speed Slabs}

The following is a rigorous derivation of the TRM for the one-speed slab problem, without delayed neutron precursors. Consider the one-dimensional, one-speed adjoint equation

$$
\begin{aligned}
-\frac{1}{v} \frac{\partial}{\partial t} \psi^{\dagger} & -\mu \frac{\partial}{\partial x} \psi^{\dagger}+\Sigma_{t}(x) \psi^{\dagger}(x, \mu, t) \\
& =\int \Sigma_{s}\left(x, \mu \rightarrow \mu^{\prime}\right) \psi^{\dagger}\left(x, \mu^{\prime}, t\right) d \mu^{\prime}+\int \bar{\nu} \Sigma_{f}(x) \chi\left(\mu^{\prime}\right) \psi^{\dagger}\left(x, \mu^{\prime}, t\right) d \mu^{\prime}
\end{aligned}
$$

where $\mu$ is the direction cosine, $x$ is the one-dimensional position, and delayed neutron precursor emission is not considered. Inserting the $\alpha$ eigenvalue, multiplying by velocity, and rearranging terms yields the one-dimensional adjoint $\alpha$-eigenvalue problem

$$
\begin{aligned}
v \mu \frac{\partial}{\partial x} \psi^{\dagger}-v \Sigma_{t}(x) \psi^{\dagger}(x, \mu)+\int v \Sigma_{s}\left(x, \mu \rightarrow \mu^{\prime}\right) \psi^{\dagger}\left(x, \mu^{\prime}\right) d \mu^{\prime} \\
+\int \bar{\nu} v \Sigma_{f}(x) \chi\left(\mu^{\prime}\right) \psi^{\dagger}\left(x, \mu^{\prime}\right) d \mu^{\prime}=\alpha \psi^{\dagger}
\end{aligned}
$$

Now, discretize the direction space into $M$ intervals between $\mu=-1$ and $\mu=+1$, where the $m$ th interval is defined as $\mu_{m-1}<\mu<\mu_{m}$. Discretize the position space into $N$ intervals between $x=x_{0}$ and $x=x_{N}$, where the $n$th interval is defined as $x_{n-1}<x<x_{n}$. Integrate the neutron importance over the $m$ th direction interval and the $n$th slab, defining this integrated importance as

$$
\psi_{n m}^{\dagger}=\int_{x_{n-1}}^{x_{n}} d x \int_{\mu_{m-1}}^{\mu_{m}} d \mu \psi^{\dagger}(x, \mu),
$$

and $\vec{\psi}^{\dagger}$ as the vector containing these importances

$$
\vec{\psi}^{\dagger}=\psi_{11}^{\dagger} \ldots \psi_{1 m}^{\dagger} \ldots \psi_{N M}^{\dagger}
$$

where the sum over the $M$ direction intervals is the scalar neutron importance

$$
\phi_{n}^{\dagger}=\sum_{m=1}^{M} \psi_{n m}^{\dagger}
$$

Integrate Eq. (3.95) over the $m$ th direction interval and $n$th slab, and multiply and divide by the integrated adjoint importance. The resulting terms are similar to the 
infinite medium problem. The collision term becomes

$$
\int_{x_{n-1}}^{x_{n}} d x \int_{\mu_{m-1}}^{\mu_{m}} d \mu v \Sigma_{t}(x) \psi^{\dagger}(x, \mu)=\left[v \Sigma_{t}(x)\right]_{n m}^{\dagger} \psi_{n m}^{\dagger}
$$

where

$$
\left[v \Sigma_{t}\right]_{n m}^{\dagger}=\frac{1}{\psi_{n m}^{\dagger}} \int_{x_{n-1}}^{x_{n}} d x \int_{\mu_{m-1}}^{\mu_{m}} d \mu v \Sigma_{t}(x) \psi^{\dagger}(x, \mu)
$$

The integral over all angles in the scattering term must be rewritten in terms of a sum

$$
\begin{aligned}
\int_{x_{n-1}}^{x_{n}} d x & \int_{\mu_{m-1}}^{\mu_{m}} d \mu \int_{-1}^{+1} d \mu^{\prime} v \Sigma_{s}\left(x, \mu \rightarrow \mu^{\prime}\right) \psi^{\dagger}\left(x, \mu^{\prime}\right) \\
& =\sum_{m^{\prime}=1}^{M} \int_{x_{n-1}}^{x_{n}} d x \int_{\mu_{m-1}}^{\mu_{m}} d \mu \int_{\mu_{m^{\prime}-1}}^{\mu_{m^{\prime}}} d \mu^{\prime} v \Sigma_{s}\left(x, \mu \rightarrow \mu^{\prime}\right) \psi^{\dagger}\left(x, \mu^{\prime}\right)
\end{aligned}
$$

and the interval-transfer rate from direction interval $m \rightarrow m^{\prime}$ of slab $n$ is defined as

$$
\left[v \Sigma_{s m \rightarrow m^{\prime}}\right]_{n}^{\dagger}=\frac{1}{\psi_{n m^{\prime}}^{\dagger}} \int_{x_{n-1}}^{x_{n}} d x \int_{\mu_{m-1}}^{\mu_{m}} d \mu \int_{\mu_{m^{\prime}-1}}^{\mu_{m^{\prime}}} d \mu^{\prime} v \Sigma_{s}\left(x, \mu \rightarrow \mu^{\prime}\right) \psi^{\dagger}\left(x, \mu^{\prime}\right) .
$$

The fission term is rewritten similarly as

$$
\begin{aligned}
\int_{x_{n-1}}^{x_{n}} d x & \int_{\mu_{m-1}}^{\mu_{m}} d \mu \int_{-1}^{+1} d \mu^{\prime} \bar{\nu} v \Sigma_{f}(x) \chi\left(\mu^{\prime}\right) \psi^{\dagger}\left(x, \mu^{\prime}\right) \\
& =\sum_{m^{\prime}=1}^{M} \int_{x_{n-1}}^{x_{n}} d x \int_{\mu_{m-1}}^{\mu_{m}} d \mu \int_{\mu_{m^{\prime}-1}}^{\mu_{m^{\prime}}} d \mu^{\prime} \bar{\nu} v \Sigma_{f}(x) \chi\left(\mu^{\prime}\right) \psi^{\dagger}\left(x, \mu^{\prime}\right)
\end{aligned}
$$

The fission-emission rate from interval $m$ to interval $m^{\prime}$ is defined as

$$
\left[\left(\bar{\nu} v \Sigma_{f}\right)_{m}(\chi)_{m^{\prime}}\right]_{n}^{\dagger}=\frac{1}{\psi_{n m^{\prime}}^{\dagger}} \int_{x_{n-1}}^{x_{n}} d x \int_{\mu_{m-1}}^{\mu_{m}} d \mu \bar{\nu} v \Sigma_{f}(x) \int_{\mu_{m^{\prime}-1}}^{\mu_{m^{\prime}}} d \mu^{\prime} \chi\left(\mu^{\prime}\right) \psi^{\dagger}\left(x, \mu^{\prime}\right)
$$

The above terms describe interactions for the same position interval: these are neutrons moving between direction intervals $m=1 \ldots M$ in slab $n$. The streaming term describes interactions between adjacent slabs. This new term in the one-dimensional adjoint problem is difficult to quantify mathematically: it is the integrated streaming term. Using Green's Theorem to redefine the integral yields

$$
\int_{x_{n-1}}^{x_{n}} d x \int_{\mu_{m-1}}^{\mu_{m}} d \mu v \mu \frac{\partial}{\partial x} \psi^{\dagger}(x, \mu)=\int_{\mu_{m-1}}^{\mu_{m}} d \mu \int_{\partial S} d S v(\mathbf{n} \cdot \mu) \psi^{\dagger}(x, \mu)
$$


where $\partial S$ describes the surface bounding the slab and $\mathbf{n}$ denotes the direction normal of the surface. In the one-dimensional problem, there are two surfaces bounding each slab at $x_{n-1}$ and $x_{n}$, with direction normals $\mathbf{n}=-1$ and $\mathbf{n}=+1$, respectively. The integral is split into

$$
-\int_{\mu_{m-1}}^{\mu_{m}} d \mu \int_{\partial S} d S v \mu \psi^{\dagger}\left(x_{n-1}, \mu\right)+\int_{\mu_{m-1}}^{\mu_{m}} d \mu \int_{\partial S} d S v \mu \psi^{\dagger}\left(x_{n}, \mu\right)
$$

a surface integral for each of the bounding surfaces. Neutrons move along the direction $\mu$ through slab $n$ (Figure 3.1): if $\mu>0$, neutrons stream in the $+x$ direction, moving from slab $n-1$ into slab $n$ at $x_{n}$, and from slab $n$ into slab $n+1$ at $x_{n+1}$; if $\mu<0$, neutrons stream in the $-x$ direction, moving from slab $n+1$ into slab $n$ at $x_{n+1}$, and from slab $n$ into slab $n-1$ at $x_{n}$. Note that there is an $m$ for which $\mu_{m}=0$ : $M$ is always even. This avoids a direction interval in which neutrons stream parallel to the faces. Including this direction interval parallel to the faces changes the computed spectrum, particularly within the continuum. To understand the behavior of the terms in the adjoint operator, first examine the term in the forward operator

$$
-\int_{x_{n-1}}^{x_{n}} d x \int_{\mu_{m-1}}^{\mu_{m}} d \mu v \mu \frac{\partial}{\partial x} \psi(x, \mu)
$$

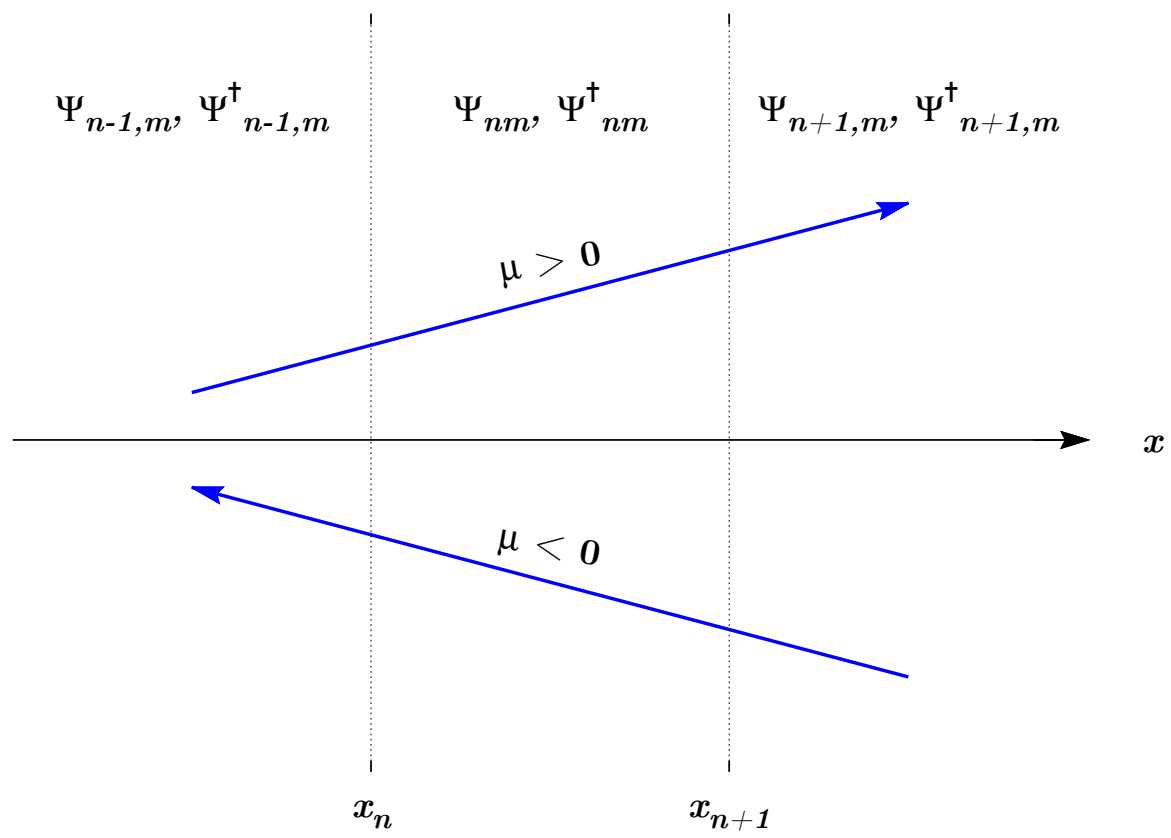

Figure 3.1: A diagram of the slab geometry shows neutrons streaming through slab $n$ with direction $\mu$. 
where the negative sign switches because of the sign of the adjoint streaming term. This integrated term looks like the net neutron current with an extra $v$ : this quantity has units of $\left[\mathrm{cm} / \mathrm{s}^{2}\right]$. Conversely, the net leakage rate $\left[\mathrm{s}^{-1}\right]$ at which neutrons flow out of slab $n$ in direction interval $m$ is

$$
\int_{x_{n-1}}^{x_{n}} d x \int_{\mu_{m-1}}^{\mu_{m}} d \mu \mu \frac{\partial}{\partial x} \psi(x, \mu) .
$$

Splitting up Eq. (3.107) with Green's Theorem yields

$$
\int_{\mu_{m-1}}^{\mu_{m}} d \mu \int_{\partial S} d S v \mu \psi\left(x_{n-1}, \mu\right)-\int_{\mu_{m-1}}^{\mu_{m}} d \mu \int_{\partial S} d S v \mu \psi\left(x_{n}, \mu\right)
$$

An extra term is necessary to obtain rates: multiplying and dividing by the incident integrated flux $\psi_{n m}$ serves this purpose. Choosing the spatial range of integration of this flux comes from the observation of the physical problem. If $\mu>0$, operate the integral at $x_{n}$ by the flux in slab $n-1$, and operate the integral at $x_{n+1}$ by the flux in slab $n$. This yields

$$
\left[v \Sigma_{L}\right]_{n-1, m} \psi_{n-1, m}-\left[v \Sigma_{L}\right]_{n^{+} m} \psi_{n m}
$$

where

$$
\begin{aligned}
{\left[v \Sigma_{L}\right]_{n-1, m} } & =\frac{1}{\psi_{n-1, m}} \int_{\mu_{m-1}}^{\mu_{m}} d \mu \int_{\partial S} d S v \mu \psi\left(x_{n-1}, \mu\right) \\
{\left[v \Sigma_{L}\right]_{n^{+} m} } & =\frac{1}{\psi_{n m}} \int_{\mu_{m-1}}^{\mu_{m}} d \mu \int_{\partial S} d S v \mu \psi\left(x_{n}, \mu\right)
\end{aligned}
$$

If $\mu<0$, operate the integral at $x_{n+1}$ by the flux in slab $n+1$, and operate the integral at $x_{n}$ by the flux in slab $n$. This yields

$$
-\left[v \Sigma_{L}\right]_{n^{-} m} \psi_{n, m}+\left[v \Sigma_{L}\right]_{n+1, m} \psi_{n+1, m}
$$

where

$$
\begin{aligned}
{\left[v \Sigma_{L}\right]_{n^{-} m} } & =\frac{1}{\psi_{n m}} \int_{\mu_{m-1}}^{\mu_{m}} d \mu \int_{\partial S} d S v \mu \psi\left(x_{n-1}, \mu\right) \\
{\left[v \Sigma_{L}\right]_{n+1, m} } & =\frac{1}{\psi_{n+1, m}} \int_{\mu_{m-1}}^{\mu_{m}} d \mu \int_{\partial S} d S v \mu \psi\left(x_{n}, \mu\right) .
\end{aligned}
$$

Now return to the adjoint problem. With a similar approach, handle the cases $\mu>0$ 
and $\mu<0$ separately. In this case operate by the exiting adjoint angular flux, because importance behaves backwards in time. This yields

$$
\begin{array}{ccc}
-\left[v \Sigma_{L}\right]_{n^{-} m}^{\dagger} \psi_{n m}^{\dagger}+\left[v \Sigma_{L}\right]_{n+1, m}^{\dagger} \psi_{n+1, m}^{\dagger}, & \text { for } & \mu>0, \\
{\left[v \Sigma_{L}\right]_{n-1, m}^{\dagger} \psi_{n-1, m}^{\dagger}-\left[v \Sigma_{L}\right]_{n^{+}, m}^{\dagger} \psi_{n^{+} m}^{\dagger},} & \text { for } \quad \mu<0,
\end{array}
$$

where

$$
\begin{aligned}
{\left[v \Sigma_{L}\right]_{n^{-} m}^{\dagger} } & =\frac{1}{\psi_{n m}^{\dagger}} \int_{\mu_{m-1}}^{\mu_{m}} d \mu \int_{\partial S} d S v \mu \psi^{\dagger}\left(x_{n-1}, \mu\right), \\
{\left[v \Sigma_{L}\right]_{n+1, m}^{\dagger} } & =\frac{1}{\psi_{n+1, m}^{\dagger}} \int_{\mu_{m-1}}^{\mu_{m}} d \mu \int_{\partial S} d S v \mu \psi^{\dagger}\left(x_{n}, \mu\right), \\
{\left[v \Sigma_{L}\right]_{n-1, m}^{\dagger} } & =\frac{1}{\psi_{n-1, m}^{\dagger}} \int_{\mu_{m-1}}^{\mu_{m}} d \mu \int_{\partial S} d S v \mu \psi^{\dagger}\left(x_{n-1}, \mu\right), \\
{\left[v \Sigma_{L}\right]_{n^{+} m}^{\dagger} } & =\frac{1}{\psi_{n m}^{\dagger}} \int_{\mu_{m-1}}^{\mu_{m}} d \mu \int_{\partial S} d S v \mu \psi^{\dagger}\left(x_{n}, \mu\right) .
\end{aligned}
$$

With these definitions, the one-speed, one-dimensional adjoint $\alpha$-eigenvalue problem is written in discretized form as

$$
\begin{aligned}
-\left[v \Sigma_{L}\right]_{n^{*} m}^{\dagger} \psi_{n m}^{\dagger}+ & {\left[v \Sigma_{L}\right]_{n^{\prime} m}^{\dagger} \psi_{n^{\prime} m}^{\dagger}-\left[v \Sigma_{t}\right]_{n m}^{\dagger} \psi_{n m}^{\dagger}+\sum_{m^{\prime}=1}^{M}\left[v \Sigma_{s m \rightarrow m^{\prime}}\right]_{n}^{\dagger} \psi_{n m^{\prime}}^{\dagger} } \\
& +\sum_{m^{\prime}=1}^{M}\left[\left(\bar{\nu} v \Sigma_{f}\right)_{m}(\chi)_{m^{\prime}}\right]_{n}^{\dagger} \psi_{n m^{\prime}}^{\dagger}=\alpha \psi_{n m}^{\dagger} .
\end{aligned}
$$

where $n^{*}$ and $n^{\prime}$ are different for the two cases defined in Eqs. (3.116) and (3.117)

The result is a matrix with two types of sections describing neutrons moving within slab $n$ between direction intervals and moving to slab $n^{\prime}$ within the same direction interval. Without delayed neutron precursors in the formulation, the matrix only encompasses prompt neutron effects described by $v\left(-\mathbf{M}+\mathbf{F}_{\mathbf{p}}\right)^{\dagger}$. Ordering the discretized phase space such that $M$ consecutive elements describe the direction intervals in the $n$th slab yields a structured matrix. Define a portion of the TRM $\mathbf{Q}^{(n)}$ as the matrix characterizing neutrons within slab $n$. The diagonal element of row $m$ 
in $\mathbf{Q}^{(n)}$ is

$$
\begin{aligned}
-\left[v \Sigma_{L}\right]_{n^{*} m}^{\dagger}- & {\left[v \Sigma_{t}\right]_{n m}^{\dagger}+\left[v \Sigma_{s m \rightarrow m}\right]_{n}^{\dagger}+\left[\left(\bar{\nu} v \Sigma_{f}\right)_{m}(\chi)_{m}\right]_{n}^{\dagger} } \\
=- & (\text { leakage rate out of slab } n)-(\text { collision rate in slab } n) \\
& +(\text { self scatter rate })+(\text { self fission-emission rate })
\end{aligned}
$$

the negative net removal rate of neutrons from direction interval $m$. The off-diagonal element of row $m$ and column $m^{\prime} \neq m$ is

$$
\begin{aligned}
{\left[v \Sigma_{s m \rightarrow m^{\prime}}\right]_{n}^{\dagger} } & +\left[\left(\bar{\nu} v \Sigma_{f}\right)_{m}(\chi)_{m^{\prime}}\right]_{n}^{\dagger} \\
& =\left(\text { scatter rate } m \rightarrow m^{\prime}\right)+\left(\text { fission-emission rate } m \rightarrow m^{\prime}\right)
\end{aligned}
$$

which is the total transition rate from direction interval $m$ to $m^{\prime}$. The remainder of the matrix is rates describing transitions to adjacent slabs. Define the matrix $\mathbf{T}^{\left(n \rightarrow n^{\prime}\right)}$, sparsely populated on the diagonal by the transition rates

$$
\left[v \Sigma_{L}\right]_{n^{\prime} m}^{\dagger}=\left(\text { leakage rate into slab } n^{\prime}\right)
$$

Because neutrons moving in the $+x$ direction do not exit to the adjacent slab to the left, and vice versa, the matrix $\mathbf{T}$ is populated with zeros on half of its diagonals. As in the infinite-medium formulation, these rates fit into the TRM interpretation, where the $i$ th state is defined as a neutron within position interval $n$ moving in direction interval $m$, where $i=M(n-1)+m$. Then, $q_{i i}$ is the net transition rate out of state $i$ and $q_{i i^{\prime}}$ is the transition rate from state $i$ to $i^{\prime}$. Neutrons transition out of state $i$ via the processes shown in Eq. (3.123). Neutrons transition into state $i^{\prime}$ of the same slab $n$ and adjacent slab $n^{\prime}$ via the processes shown in Eq. (3.124) and Eq. (3.125), respectively. The full TRM for the one-dimensional geometry with vacuum boundaries takes the form

$$
\mathbf{Q}=\left[\begin{array}{ccc}
\mathbf{Q}^{(1)} & \mathbf{T}^{(1 \rightarrow 2)} & \\
\mathbf{T}^{(2 \rightarrow 1)} & \ddots & \ddots \\
& \ddots & \mathbf{Q}^{(N)}
\end{array}\right]
$$

where $N$ is the total number of slabs. The TRM is considerably sparse because neutrons only transition to the adjacent slabs, in either the positive or negative direction. In total, the size of the matrix is $N M \times N M$. However, due to the structure mentioned, the absolute maximum number of non-zero elements of the $N^{2} M^{2}$ of the 
TRM is $N M^{2}+M(N-1)$. Upon leakage or absorption, neutrons transition to a absorbing state undefined within the matrix formulation. As for the infinite-medium formulation, the diagonal elements of the matrix do not satisfy Eq. (3.3).

\section{Continuous-Energy Slabs}

Including the energy space in multigroup or continuous-energy one-dimensional problems is a combination of the one-speed and the infinite-medium continuous-energy formulations: the complete derivation is not shown here, but some comments about the structure of the matrix and the difference in the transition rates are worth mentioning. The maximum number of non-zero elements of the $N^{2}(G M+J)^{2}$ is $N(G M+J)^{2}+G M(N-1)$, though depending on the energy discretization, this is likely significantly less.

The rates describing neutrons transferring between the $M$ direction intervals within slab $n$ expand to include energy. In both fission and scattering events, neutrons enter the event at $E, \hat{\boldsymbol{\Omega}}$ and exit at $E^{\prime}, \hat{\boldsymbol{\Omega}}^{\prime}$. Thus, the $\mathbf{Q}^{(n)}$ matrix for slab $n$ is of size $G M \times G M$ where the diagonal elements are net removal rates from interval $g$, $m$ and the off-diagonal elements are transition rates from interval $g, m$ to $g^{\prime}, m^{\prime}$. If delayed fission is included, the $\mathbf{Q}^{(n)}$ matrix extends to include $J$ more states describing delayed neutron precursor behavior. The $\mathbf{T}^{\left(n \rightarrow n^{\prime}\right)}$ matrices have similar structure as for the one-speed case. For stationary fuel, the portion of the $\mathbf{T}$ matrix describing precursors transitioning to adjacent slabs is zero. This maintains the TRM form in Eq. (3.126).

\subsubsection{Multi-Dimensional Media}

For two- and three-dimensional media, the TRM does not change significantly. It includes more matrices $\mathbf{T}^{\left(n \rightarrow n^{\prime}\right)}$ dependent on the discretized position space. The number of these matrices is equal to the number of position intervals to which a neutron possibly leaks, or the number of adjacent position intervals. In two dimensions, a triangle, square, and hexagon have three, four, and six adjacent neighbors, respectively. In three dimensions, a triangular prism, hexahedron, and hexagonal prism have five, six, and eight adjacent neighbors, respectively. Because the direction phase space is also discretized, the number of non-zero matrices $\mathbf{T}^{\left(n \rightarrow n^{\prime}\right)}$ is likely fewer than the number of adjacent neighbors, e.g., neutrons that transfer to an adjacent position state remain in the same direction interval.

The transition rates change very little from the previous cases, but it is still useful 
to define them in the general sense. First, define the integrated flux

$$
\psi_{n g m}^{\dagger}=\left\langle\psi^{\dagger}(\mathbf{r}, E, \hat{\mathbf{\Omega}})\right\rangle_{n g m}=\int_{\partial V_{n}} d \mathbf{r} \int_{E_{g+1}}^{E_{g}} d E \int_{\hat{\boldsymbol{\Omega}}_{m-1}}^{\hat{\boldsymbol{\Omega}}_{m}} d \boldsymbol{\Omega} \psi^{\dagger}(\mathbf{r}, E, \hat{\boldsymbol{\Omega}}) .
$$

Then, the transition rates become

$$
\begin{aligned}
{\left[v \Sigma_{t}\right]_{n g m}^{\dagger} \psi_{n g m}^{\dagger} } & =\left\langle v \Sigma_{t}(\mathbf{r}, E) \psi^{\dagger}(\mathbf{r}, E, \hat{\mathbf{\Omega}})\right\rangle_{n g m}, \\
{\left[v_{g} \Sigma_{s g m \rightarrow g^{\prime} m^{\prime}}\right]_{n}^{\dagger} \psi_{n g^{\prime} m^{\prime}}^{\dagger} } & \left\langle v\left\langle\Sigma_{s}\left(\mathbf{r} ; E, \hat{\mathbf{\Omega}} \rightarrow E^{\prime}, \hat{\boldsymbol{\Omega}}^{\prime}\right) \psi^{\dagger}\left(\mathbf{r}, E^{\prime}, \hat{\mathbf{\Omega}}^{\prime}\right)\right\rangle_{g^{\prime} m^{\prime}}\right\rangle_{n g m}, \\
{\left[\left(\bar{\nu} v \Sigma_{f}\right)_{g m}(\chi)_{g^{\prime} m^{\prime}}\right]_{n}^{\dagger} \psi_{n g^{\prime} m^{\prime}}^{\dagger} } & \\
= & \left\langle\bar{\nu}(E) v \Sigma_{f}(\mathbf{r}, E)\left\langle\frac{\chi\left(E^{\prime}\right)}{4 \pi} \psi^{\dagger}\left(\mathbf{r}, E^{\prime}, \hat{\mathbf{\Omega}}^{\prime}\right)\right\rangle_{g^{\prime} m^{\prime}}\right\rangle_{n g m}, \\
{\left[v \Sigma_{L}\right]_{n g m}^{\dagger} \psi_{n g m}^{\dagger} } & =\left\langle\int_{\partial S_{\text {in }}} d S_{\text {in }}(\hat{\mathbf{\Omega}} \cdot \mathbf{n}) v \psi^{\dagger}(\mathbf{r}, E, \hat{\mathbf{\Omega}})\right\rangle_{g m}, \\
{\left[v \Sigma_{L}\right]_{n^{\prime} g m}^{\dagger} \psi_{n^{\prime} g m}^{\dagger} } & =\left\langle\int_{\partial S_{\text {out }}} d S_{\text {out }}(\hat{\mathbf{\Omega}} \cdot \mathbf{n}) v \psi^{\dagger}(\mathbf{r}, E, \hat{\mathbf{\Omega}})\right\rangle_{g m},
\end{aligned}
$$

where $S_{\text {in }}$ and $S_{\text {out }}$ specify the surfaces at which neutrons in direction $m$ stream into and out of position interval $n$. The TRM for the multi-dimensional case maintains the general format of the one-dimensional case in Eq. (3.126), with the additional $\mathbf{T}^{\left(n \rightarrow n^{\prime}\right)}$ matrices

$$
\mathbf{Q}=\left[\begin{array}{cccc}
\mathbf{Q}^{(1)} & \mathbf{T}^{(1 \rightarrow 2)} & \mathbf{T}^{(1 \rightarrow 3)} & \ldots \\
\mathbf{T}^{(2 \rightarrow 1)} & \mathbf{Q}^{(2)} & \ddots & \\
\mathbf{T}^{(3 \rightarrow 1)} & \ddots & \ddots & \\
\vdots & & &
\end{array}\right]
$$

\subsection{Transition Rate Calculations}

There are no approximations in the formulation of the discretized problem, but quantities are weighted by the adjoint importance, as denoted by the ${ }^{\dagger}$ notation, which is unavailable in most Monte Carlo codes. An introduced approximation results from weighting the rates with forward quantities instead of the adjoint importance, $\psi^{\dagger}(\mathbf{r}, \hat{\Omega}, E)$. This enables the rates to be calculated during a forward Monte Carlo calculation and is beneficial because the main interest is in the forward problem, as its eigenfunctions determine the neutron flux. Also, the elements of the discretized 
forward $\alpha$-eigenvalue problem are weighted by the forward flux. This also helps maintain the relationship in Eq. (3.49) for a discretized phase space. This section shows the relationship between the forward- and adjoint-weighted rates and the forwardweighted approximations using the infinite-medium continuous-energy problem as an example.

\subsubsection{Rate Approximations}

Consider the forward infinite-medium $\alpha$-eigenvalue problem, in contrast to the adjoint problem in Eqs. (3.68) and (3.69),

$$
\begin{gathered}
-v \Sigma \psi(E)+\int v \Sigma_{s}\left(E^{\prime} \rightarrow E\right) \psi\left(E^{\prime}\right) d E^{\prime}+\int(1-\beta) \bar{\nu}\left(E^{\prime}\right) v \Sigma_{f}\left(E^{\prime}\right) \chi_{p}(E) \psi\left(E^{\prime}\right) d E^{\prime} \\
+\sum_{j=1}^{J} v \chi_{j}(E) \lambda_{j} C_{j}=\alpha \psi \\
-\lambda_{j} C_{j}+\int \beta_{j} \bar{\nu}\left(E^{\prime}\right) \Sigma_{f}\left(E^{\prime}\right) \psi\left(E^{\prime}\right) d E^{\prime}=\alpha C_{j}
\end{gathered}
$$

Using the same approach in discretizing the energy space in Eqs. (3.70) through (3.82) for the adjoint problem, the discretized forward problem is written as

$$
\begin{aligned}
&-\left(v \Sigma_{t}\right)_{g} \psi_{g}+\sum_{g^{\prime}=1}^{G}\left(v_{g} \Sigma_{s g^{\prime} \rightarrow g}\right) \psi_{g^{\prime}}+\sum_{g^{\prime}=1}^{G}\left(\bar{\nu}_{p} \Sigma_{f}\right)_{g^{\prime}}\left(v \chi_{p}\right)_{g} \psi_{g^{\prime}} \\
&+\sum_{j=1}^{J}\left(v \chi_{j}\right)_{g} \lambda_{j} C_{j}=\alpha \psi_{g}, \\
&-\lambda_{j} C_{j}+\sum_{g^{\prime}=1}^{G} \beta_{j}\left(\bar{\nu}_{d} \Sigma_{f}\right)_{g^{\prime}} \psi_{g^{\prime}}=\alpha C_{j},
\end{aligned}
$$

where the loss of the ${ }^{\dagger}$ notation on the constants is due to the weighting with the forward flux. A comparison of these forward constants with those of the adjoint problem (Table 3.1) shows the incentive to obtain the adjoint constants: they are more physical quantities, e.g., the emission spectra for the forward problem includes the neutron speed $v$, while most of the adjoint quantities are rates. But, if the TRMM weights the forward constants with the forward flux and the adjoint constants with the adjoint flux, the resulting discretized operator matrices are not related by Eq. (3.49), and their respective eigenfunctions are not bi-orthogonal.

Without knowledge of the adjoint importance, the forward neutron concentrations 
Table 3.1: True weighted constants of the discretized forward and adjoint $\alpha$-eigenvalue infinite medium problems.

\begin{tabular}{lcc}
\hline Parameter & Forward & Adjoint \\
\hline Collision & $\frac{\langle v \Sigma(E) \psi(E)\rangle_{g}}{\langle\psi(E)\rangle_{g}}$ & $\frac{\left\langle v \Sigma(E) \psi^{\dagger}(E)\right\rangle_{g}}{\left\langle\psi^{\dagger}(E)\right\rangle_{g}}$ \\
Inscatter & $\frac{\left\langle v\left\langle\Sigma_{s}\left(E^{\prime} \rightarrow E\right) \psi\left(E^{\prime}\right)\right\rangle_{g^{\prime}}\right\rangle_{g}}{\left\langle\psi\left(E^{\prime}\right)\right\rangle_{g^{\prime}}}$ & $\frac{\left\langle v\left\langle\Sigma_{s}\left(E \rightarrow E^{\prime}\right) \psi^{\dagger}\left(E^{\prime}\right)\right\rangle_{g^{\prime}}\right\rangle_{g}}{\left\langle\psi^{\dagger}\left(E^{\prime}\right)\right\rangle_{g^{\prime}}}$ \\
Prompt Fission & $\frac{\left\langle(1-\beta) \bar{\nu}\left(E^{\prime}\right) \Sigma_{f}\left(E^{\prime}\right) \psi\left(E^{\prime}\right)\right\rangle_{g^{\prime}}}{\left\langle\psi\left(E^{\prime}\right)\right\rangle_{g^{\prime}}}$ & $\left\langle(1-\beta) \bar{\nu}(E) v \Sigma_{f}(E)\right\rangle_{g}$ \\
Delayed Fission & $\frac{\left\langle\beta \bar{\nu}\left(E^{\prime}\right) \Sigma_{f}\left(E^{\prime}\right) \psi\left(E^{\prime}\right)\right\rangle_{g^{\prime}}}{\left\langle\psi\left(E^{\prime}\right)\right\rangle_{g^{\prime}}}$ & $\left\langle\beta \bar{\nu}(E) v \Sigma_{f}(E)\right\rangle_{g}$ \\
Prompt Fission & $\left\langle v \chi_{p}(E)\right\rangle_{g}$ & $\frac{\left\langle\chi_{p}\left(E^{\prime}\right) \psi^{\dagger}\left(E^{\prime}\right)\right\rangle_{g^{\prime}}}{\left\langle\psi^{\dagger}\left(E^{\prime}\right)\right\rangle_{g^{\prime}}}$ \\
Emission & $\frac{\left\langle\chi_{j}\left(E^{\prime}\right) \psi^{\dagger}\left(E^{\prime}\right)\right\rangle_{g^{\prime}}}{\left\langle\psi^{\dagger}\left(E^{\prime}\right)\right\rangle_{g^{\prime}}}$ \\
Delayed Fission & $\frac{\left\langle\frac{1}{v} \psi \psi^{\dagger}(E)\right\rangle_{g}}{\left\langle\psi_{j}^{\dagger}(E)\right\rangle_{g}}$ \\
Emission & $\langle\psi(E)\rangle_{g}$ & $\frac{\left\langle\psi^{\prime}\right.}{\text { Inverse Speed }}$ \\
\hline
\end{tabular}

serve as the weighting function for the adjoint constants. This selected weighting function facilitates the use of Monte Carlo tallies and matches best to the elements of the adjoint operator. Consider an alternate formulation of the forward $\alpha$-eigenvalue problem in Eqs. (3.134) and (3.135) by separating the neutron angular flux into its speed and neutron concentration $n(E)$ components

$$
\begin{gathered}
-v \sum n(E)+\int v^{\prime} \Sigma_{s}\left(E^{\prime} \rightarrow E\right) n\left(E^{\prime}\right) d E^{\prime}+\int(1-\beta) \bar{\nu}\left(E^{\prime}\right) v^{\prime} \Sigma_{f}\left(E^{\prime}\right) \chi_{p}(E) n\left(E^{\prime}\right) d E^{\prime} \\
+\sum_{j=1}^{J} \chi_{j}(E) \lambda_{j} C_{j}=\alpha n \\
-\lambda_{j} C_{j}+\int \beta_{j} \bar{\nu}\left(E^{\prime}\right) v^{\prime} \Sigma_{f}\left(E^{\prime}\right) n\left(E^{\prime}\right) d E^{\prime}=\alpha C_{j}
\end{gathered}
$$

The general matrix form of this problem is

$$
\left[\begin{array}{cc}
-\mathbf{M}+\mathbf{F}_{\mathbf{p}} & \boldsymbol{\chi} \boldsymbol{\lambda} \\
\mathbf{F}_{\mathbf{d}} & -\boldsymbol{\lambda}
\end{array}\right]\left[\begin{array}{ll}
v & 0 \\
0 & I
\end{array}\right]\left[\begin{array}{l}
n \\
\boldsymbol{C}
\end{array}\right]=\alpha\left[\begin{array}{c}
n(\mathbf{r}, E, \hat{\mathbf{\Omega}}) \\
\boldsymbol{C}(\mathbf{r})
\end{array}\right]
$$


where the product of the left two matrices is the transpose of the TRM. Utilizing this relation, the forward-weighted constants within the matrix are used to approximate the TRM. Following a similar methodology as for the adjoint problem in Eqs. (3.70) through (3.82) yields

$$
\begin{aligned}
&-\left(v \Sigma_{t}\right)_{g}^{n} n_{g}+\sum_{g^{\prime}=1}^{G}\left(v_{g^{\prime}} \Sigma_{s g^{\prime} \rightarrow g}\right)^{n} n_{g^{\prime}}+\sum_{g^{\prime}=1}^{G}\left(\bar{\nu}_{p} v \Sigma_{f}\right)_{g^{\prime}}^{n} \chi_{p g} n_{g^{\prime}} \\
&+\sum_{j=1}^{J} \chi_{j g} \lambda_{j} C_{j}=\alpha n_{g}, \\
&-\lambda_{j} C_{j}+\sum_{g^{\prime}=1}^{G} \beta_{j}\left(\bar{\nu}_{d} v \Sigma_{f}\right)_{g^{\prime}}^{n} n_{g^{\prime}}=\alpha C_{j},
\end{aligned}
$$

where the ${ }^{n}$ notation denotes constants weighted by neutron concentrations. These constants serve as an approximation to the adjoint-weighted constants for the infinitemedium problem (Table 3.2) . For the collision rate, the neutron concentration replaces the importance as the weighting functions. In the adjoint-weighted quantities,

Table 3.2: Approximated forward-weighted constants for the discretized adjoint $\alpha$ eigenvalue infinite medium problems.

\begin{tabular}{ccc}
\hline Parameter & True Adjoint & Forward-weighted Approximation \\
\hline$\left(v \Sigma_{t}\right)_{g}^{\dagger}$ & $\frac{\left\langle v \Sigma(E) \psi^{\dagger}(E)\right\rangle_{g}}{\left\langle\psi^{\dagger}(E)\right\rangle_{g}}$ & $\frac{\langle v \Sigma(E) n(E)\rangle_{g}}{\langle n(E)\rangle_{g}}$ \\
$\left(v_{g} \Sigma_{s g \rightarrow g^{\prime}}\right)^{\dagger}$ & $\frac{\left\langle v\left\langle\Sigma_{s}\left(E \rightarrow E^{\prime}\right) \psi^{\dagger}\left(E^{\prime}\right)\right\rangle_{g^{\prime}}\right\rangle_{g}}{\left\langle\psi^{\dagger}\left(E^{\prime}\right)\right\rangle_{g^{\prime}}}$ & $\frac{\left\langle v\left\langle\Sigma_{s}\left(E \rightarrow E^{\prime}\right) n(E)\right\rangle_{g^{\prime}}\right\rangle_{g}}{\langle n(E)\rangle_{g}}$ \\
$\left(\bar{\nu}_{p} v \Sigma_{f}\right)_{g}^{\dagger}$ & $\left\langle(1-\beta) \bar{\nu}(E) v \Sigma_{f}(E)\right\rangle_{g}$ & $\frac{\left\langle(1-\beta) \bar{\nu}(E) v \Sigma_{f}(E) n(E)\right\rangle_{g}}{\langle n(E)\rangle_{g}}$ \\
$\left(\bar{\nu}_{d} v \Sigma_{f}\right)_{g}^{\dagger}$ & $\left\langle\beta \bar{\nu}(E) v \Sigma_{f}(E)\right\rangle_{g}$ & $\frac{\left\langle\beta \bar{\nu}(E) v \Sigma_{f}(E) n(E)\right\rangle_{g}}{\langle n(E)\rangle_{g}}$ \\
$\chi_{p g^{\prime}}^{\dagger}$ & $\frac{\left\langle\chi_{p}\left(E^{\prime}\right) \psi^{\dagger}\left(E^{\prime}\right)\right\rangle_{g^{\prime}}}{\left\langle\psi^{\dagger}\left(E^{\prime}\right)\right\rangle_{g^{\prime}}}$ & $\left\langle\chi_{p}\left(E^{\prime}\right)\right\rangle_{g^{\prime}}$ \\
$\chi_{j g^{\prime}}^{\dagger}$ & $\frac{\left\langle\chi_{j}\left(E^{\prime}\right) \psi^{\dagger}\left(E^{\prime}\right)\right\rangle_{g^{\prime}}}{\left\langle\psi^{\dagger}\left(E^{\prime}\right)\right\rangle_{g^{\prime}}}$ & $\left\langle\chi_{j}\left(E^{\prime}\right)\right\rangle_{g^{\prime}}$ \\
$\frac{1}{v_{v}^{\dagger}}$ & $\left.\frac{1}{v} \psi^{\dagger}(E)\right\rangle_{g}$ & $\langle n(E)\rangle_{g}$ \\
$\left\langle\psi^{\dagger}(E)\right\rangle_{g}$ & \\
\hline$v n(E)\rangle_{g}$
\end{tabular}


the emission spectra are weighted by the adjoint importance and the fission rates are not weighted. In the forward-weighted approximation, the opposite is true. The emission probabilities are

$$
\begin{aligned}
& \left\langle\chi_{p}\left(E^{\prime}\right)\right\rangle_{g^{\prime}}=\int_{E_{g^{\prime}+1}}^{E_{g^{\prime}}} d E^{\prime} \chi_{p}\left(E^{\prime}\right), \\
& \left\langle\chi_{j}\left(E^{\prime}\right)\right\rangle_{g^{\prime}}=\int_{E_{g^{\prime}+1}}^{E_{g^{\prime}}} d E^{\prime} \chi_{j}\left(E^{\prime}\right),
\end{aligned}
$$

where the $\chi$-spectra are assumed to be normalized. The adjoint-weighted inscattering rate is weighted by the adjoint importance of outgoing neutrons. In the forwardweighted approximation, it is weighted by the neutron concentration of the incoming neutrons. These approximations extend to include the position and direction phase space.

Tallying the inverse velocity among the phase space is necessary to obtain the forward matrix. The TRM is built with the forward-weighted rates. To obtain the linear transport operator, each row $i$ of the TRM is multiplied by the inverse speed in state $i$. The matrix is then transposed and each row $j$ of the TRM is multiplied by the speed in state $j$. This sequence is shown in Eq. (3.59). For sparsely stored matrices, the matrix does not necessarily need to be transposed in memory, as a change in the storage scheme has the same affect. For this case, the appropriate elements are operated on by the velocity.

\subsubsection{Monte Carlo Tallies}

Tallies accumulated during the forward random walk yields transition rates and probabilities that are used to build the TRM. Monte Carlo tracks neutrons in the continuous position-energy-direction phase space, and uses physical definitions to relate tallies to mathematical quantities [9]. For example, the scalar flux is the total path length traversed per unit time per unit volume by all particles [10]. Mathematically, this is

$$
\phi(\vec{r}) d V=\text { rate at which particles generate path length in } d V \text { about } \vec{r}
$$

where $d V$ is a differential unit of volume. Integrating this over some arbitrary volume $V$ and dividing by that volume yields

$$
\bar{\phi}_{V}=\frac{1}{V} \int_{V} \phi(\vec{r}) d V=\frac{1}{V}(\text { total path length by particles in } V)
$$


the volume-averaged flux. During the Monte Carlo random walk, tallies estimate this quantity by summing track lengths in an arbitrary volume and energy interval,

$$
\bar{\phi}_{V}=\frac{1}{V} \frac{1}{N} \sum_{i} l_{i}
$$

where $N$ is the total number of neutron histories and $l_{i}$ is the track length of a single history. A similar approach yields the weighted rates.

Many of the forward-weighted estimates of the adjoint-weighted rates have a common denominator. In terms of the neutron flux, this is

$$
\langle n(\mathbf{r}, E, \hat{\mathbf{\Omega}})\rangle_{n g m}=\left\langle\frac{1}{v} \psi(\mathbf{r}, E, \hat{\mathbf{\Omega}})\right\rangle_{n g m}
$$

In a similar way as for the volume-averaged flux, the Monte Carlo estimate is the sum of track times, i.e., track length divided by $v$, within a given position, energy, and direction interval

$$
\langle n(\mathbf{r}, E, \hat{\Omega})\rangle_{n g m}=\frac{1}{N} \sum_{i} t_{i}
$$

where $t_{i}$ is the time of the individual tracks. An alternate interpretation of this quantity thats fits with the TRMM is that $N$ is the number of events that end an individual track and $\sum_{i} t_{i}$ is the total amount of time spent by neutrons within the $n, g, m$ interval. Events that end a track are collisions or leakage from the volume element. With these definitions, the denominator is the mean time between events and the inverse of this denominator is the frequency with which events occur, or the event rate.

The remaining parts of the tallies are probabilities of specific events ending a neutron track. For example, the numerator of the collision rate is

$$
\langle v \Sigma(\mathbf{r}, E) n(\mathbf{r}, E, \hat{\mathbf{\Omega}})\rangle_{n g m}=\langle\Sigma(\mathbf{r}, E) \psi(\mathbf{r}, E, \hat{\mathbf{\Omega}})\rangle_{n g m}
$$

which is the total number of reactions that occur in the $n, g, m$ interval per particle. Physically, this is the probability that the event that ends a track is a collision, and the Monte Carlo estimate is

$$
\langle v \Sigma(\mathbf{r}, E) n(\mathbf{r}, E, \hat{\mathbf{\Omega}})\rangle_{n g m}=\frac{\text { total number of collisions }}{N},
$$


where $N$ is the total number of events. This is conveniently written as a probability

$$
\langle v \Sigma(\mathbf{r}, E) n(\mathbf{r}, E, \hat{\mathbf{\Omega}})\rangle_{n g m}=p_{\text {collision }}
$$

where $p_{\text {collision }}$ is the probability that an event ends in a collision. Combining this probability with the event rate, the Monte Carlo estimate of the collision rate in the $n, g, m$ interval becomes

$$
\frac{\langle v \Sigma(\mathbf{r}, E) n(\mathbf{r}, E, \hat{\mathbf{\Omega}})\rangle_{n g m}}{\langle n(\mathbf{r}, E, \hat{\mathbf{\Omega}})\rangle_{n g m}}=\frac{p_{\text {collision }}}{\frac{1}{N} \sum_{i} t_{i}} .
$$

This analysis extends to the other reaction rates (Table 3.3) in the TRM elements. The Monte Carlo estimate of leakage probabilities are sums of the number of neutrons crossing a surface

$$
\left\langle\int_{\partial S_{\text {out }}} d S_{\text {out }}(\hat{\mathbf{\Omega}} \cdot \mathbf{n}) n(\mathbf{r}, E, \hat{\mathbf{\Omega}})\right\rangle_{g m}=\frac{1}{N} \sum_{i} n_{i}
$$

Table 3.3: Monte Carlo transition rate estimates for the general multidimensional problem.

\begin{tabular}{cc}
\hline Parameter & Monte Carlo Tally Estimate ${ }^{\mathrm{a}, \mathrm{b}}$ \\
\hline$\left(v \Sigma_{t}\right)_{n g m}^{\dagger}$ & $\left(p_{\text {collision }}\right)_{n g m}\left(\frac{1}{N} \sum_{i} t_{i}\right)_{n g m}^{-1}$ \\
$\left(v_{g} \Sigma_{s g m \rightarrow g^{\prime} m^{\prime}}\right)_{n}^{\dagger}$ & $\left(p_{\text {scatter to } g^{\prime} m^{\prime}}\right)_{n g m}\left(\frac{1}{N} \sum_{i} t_{i}\right)_{n g m}^{-1}$ \\
$\left(\bar{\nu}_{p} v \Sigma_{f}\right)_{n g m}^{\dagger}$ & $\left(\bar{\nu}_{p} p_{\text {fission }}\right)_{n g m}\left(\frac{1}{N} \sum_{i} t_{i}\right)_{n g m}^{-1}$ \\
$\left(\bar{\nu}_{d} v \Sigma_{f}\right)_{n g m}^{\dagger}$ & $\left(\bar{\nu}_{d} p_{\text {fission }}\right)_{n g m}\left(\frac{1}{N} \sum_{i} t_{i}\right)_{n g m}^{-1}$ \\
$\left(v \Sigma_{L}\right)_{n g m}^{\dagger}$ & $\left(p_{\text {leakage to } n^{\prime}}\right)_{n g m}\left(\frac{1}{N} \sum_{i} t_{i}\right)_{n g m}^{-1}$ \\
$\chi_{p n g^{\prime} m^{\prime}}^{\dagger}$ & $\left(p_{\text {emission }}\right)_{n g^{\prime} m^{\prime}}$ \\
$\chi_{j n g^{\prime} m^{\prime}}^{\dagger}$ & $\left(p_{j, \text { emission }}\right)_{n g^{\prime} m^{\prime}}$ \\
$\frac{1}{v_{n g m}^{\dagger}}$ & $\left(\frac{1}{N} \sum_{i} t_{i}\right)_{n g m}\left(\frac{1}{N} \sum_{i} l_{i}\right)_{n g m}^{-1}$ \\
\hline
\end{tabular}

${ }^{\mathrm{a}} p_{x}$ is the probability of event $x$.

b $(\cdot)_{x}$ denotes the position, energy, and direction interval of the tally. 
where $n_{i}$ is the number of passages out through the exiting surface for track $i$. This quantity becomes the probability of leakage

$$
\frac{1}{N} \sum_{i} n_{i}=\frac{\text { total number of leakages }}{N}=p_{\text {leakage to } n^{\prime}}
$$

The integrated probabilities for neutron emission from fission are simple number ratios

$$
\left\langle\frac{\chi\left(E^{\prime}\right)}{4 \pi}\right\rangle_{n g^{\prime} m^{\prime}}=\frac{\text { total number of emissions to } g^{\prime}, m^{\prime}}{N_{\mathrm{f}}}=p_{\text {emission }}
$$

where $N_{\mathrm{f}}$ is the total number of fissions. The average number of prompt and delayed neutrons emitted per fission are tallied when banking fission neutrons. Finally, the track length tally used in the calculation of the neutron speed is

$$
\langle v n(\mathbf{r}, E, \hat{\mathbf{\Omega}})\rangle_{n g m}=\frac{1}{N} \sum_{i} l_{i}
$$

The assertion is that in the limit of continuous position-energy-direction intervals, the forward-weighted rates are equivalent to those obtained with continuous-energy adjoint Monte Carlo.

\subsection{Eigenvalue Algorithms}

Before generating expanded solutions, the TRMM determines the eigenpairs of the TRM and the linear transport operator. Several methods for obtaining eigenvalues are available, each with different results. The power method finds the dominant, or largest, eigenvalue and its associated eigenfunction. The inverse power method determines the smallest eigenvalue or the eigenvalue closest to a given value, with its associated eigenfunction. Deflation methods find sets of eigenvalues by removing components of already-determined eigenvalues [11]. Some of these methods are considered relatively ineffective for general use due to them being very slow. The methods for determining $\alpha$ eigenvalues discussed in $\S 2.5$ use some of these eigenvalue methods.

Algorithms based on eigenvalue-revealing factorizations of matrices are an improvement over these simpler algorithms. In the factorization, eigenvalues and eigenfunctions appear as the entries in some of the factors. For large matrices, these factorizations become too costly to compute, and they often destroy the structure of sparse matrices [12]. In these cases, iterative methods are more useful for determining 
some eigenpairs of the matrix.

Though the TRMM uses routines found in standard linear algebra packages, it is not suitable to use them as generic black boxes, but to have some understanding of the methodology in order to utilize them fully. The methods discussed in this section are not all-inclusive, focusing on the methods that the TRMM uses.

\subsubsection{Eigenvalue-Revealing Factorizations}

The following factorizations of the $m \times m$ square matrix $A$ are based on the idea of introducing zeros to the matrix by applying a sequence of transformations to the original matrix. The eigenvalue decomposition of $A$ is

$$
A=X \Lambda X^{-1}
$$

where $X$ is nonsingular and $\Lambda$ is diagonal. Rewriting this as the eigenvalue problem

$$
A X=X \Lambda
$$

shows that the $i$ th diagonal element of $\Lambda$ and the $i$ th column of $X$ are an associated eigenpair of $A$. If the algebraic multiplicity of an eigenvalue of $A$ exceeds its geometric multiplicity, i.e., if several eigenvalues $\lambda$ share the same eigenfunction, then the eigenvalue is said to be defective. Then, $A$ is a defective matrix and this factorization does not exist because the matrix does not have a complete basis of eigenfunctions [12].

The unitary diagonalization of $A$ is

$$
A=U \Lambda U^{*}
$$

where $U$ is unitary, i.e., $U^{-1}=U^{*}$, and the notation * denotes the conjugate transpose. The elements of $\Lambda$ and $U$ share the same behavior as for the eigenvalue decomposition, and this factorization only exists if $A$ is normal, i.e., $A^{*} A=A A^{*}$.

The Schur factorization of $A$ is

$$
A=U T U^{*}
$$

where $T$ is upper-triangular. Because $A$ and $T$ are similar, the eigenvalues of $A$ appear along the diagonal of $T$. The eigenfunctions appear in $U$, the matrix of Schur vectors. All matrices have a Schur factorization, and for this reason, many methods targeting eigenvalue-revealing factorizations utilize the Schur form. Also, unitary 
transformation algorithms tend to be numerically stable. Both MATLAB [13] and LAPACK [14] use some algorithm to compute the Schur form of a matrix.

Now that the factorization is known, it is left to develop algorithms for computing the Schur form of the matrix $A$. Often the worst and the best algorithms require vastly different numbers of computations. Consider the basic QR algorithm, based on the QR decomposition

$$
A=Q R
$$

where $Q$ is an orthogonal matrix and $R$ is upper-triangular. Then, the $\mathrm{QR}$ algorithm for computing the Schur form of $A$ proceeds as follows:

- Set $A^{(0)}=A, U^{(0)}=I$, and $k=1$.

- Compute the QR decomposition $A^{(k-1)}=Q^{(k)} R^{(k)}$.

- Update the matrix for the next iteration $A^{(k)}=R^{(k)} Q^{(k)}$.

- Update the value of the total transformation matrix $U^{(k)}=U^{(k-1)} Q^{(k)}$.

This algorithm is repeated until $A^{(k)}$ is the upper-triangular matrix $T$, and $U^{(k)}$ is converged to the matrix of Schur vectors. While this theoretically works, is it far too computationally costly to be practical, requiring $O\left(m^{4}\right)$ or more flops [15]. First reducing the matrix $A$ to the Hessenberg form

$$
A=Q H Q^{*}
$$

then performing the $\mathrm{QR}$ algorithm to reduce the matrix to upper-triangular form decreases the computational demand to $O\left(\mathrm{~m}^{3}\right)$ flops. Householder reduction to Hessenberg form has zeros below the first sub-diagonal

$$
\left[\begin{array}{ccccc}
\times & \times & \times & \times & \times \\
\times & \times & \times & \times & \times \\
& \times & \times & \times & \times \\
& & \times & \times & \times \\
& & & \times & \times
\end{array}\right]
$$

A further improvement on the QR algorithm using well-chosen shifts has been the standard method for computing all eigenvalues of a matrix, until more recent divideand-conquer algorithms [12]. This method applies best to dense or full matrices because for sparse matrices, reduction to Hessenberg form fills in elements that were previously zero, destroying the sparsity of the matrix [15]. If the sparse matrix is significantly large, the memory demand of this method becomes prohibitive. 


\subsubsection{Iterative Methods}

Iterative eigenvalue methods address two shortcomings of direct factorization schemes: (i) for large matrices, the $O\left(\mathrm{~m}^{3}\right)$ flops required to factorize the matrix becomes incredibly large, and (ii) the structure of sparse matrices are subject to fill-in during reduction to Hessenberg form. Instead of computing the full spectrum in $O\left(\mathrm{~m}^{3}\right)$ flops, iterative methods converge geometrically to a solution, where the residual is always non-zero. While these methods do not deliver exact answers, consider that even direct methods are inexact when carried out by a computer: they deliver results at no better than machine precision. Iterative methods often deliver results to machine precision in fewer operations, dependent on the spectral properties of the matrix. Ideal iterative methods have speedups from $O\left(\mathrm{~m}^{3}\right)$ to $O(\mathrm{~m})$, but for typical problems, this speedup is more like $O\left(\mathrm{~m}^{3}\right)$ to $O\left(\mathrm{~m}^{2}\right)$ : as problems become larger, the speedup is increased [12]. The Lanczos iteration applies to finding eigenvalues of Hermitian matrices $A=A^{*}$, but the linear transport operator does not exhibit this property, so the TRMM uses the Arnoldi iteration. The linear algebra package ARPACK [16] uses Arnoldi iterative methods to solve for eigenvalues and eigenfunctions of large sparse matrices. Several iterative methods are also available for solving fixed-source problems.

\section{Arnoldi Iteration}

The goal of the Arnoldi iteration is to transform the matrix $A$ into the reduced Hessenberg form

$$
A=Q_{n} H_{n} Q_{n}^{*}
$$

and to relate the eigenpairs of the resulting square matrices $A$ and $H_{n}$ as

$$
A Q_{n} u=Q_{n} H_{n} u=\lambda Q_{n} u
$$

The eigenvalues of $H_{n}$ are known as Arnoldi eigenvalue estimates or Ritz values, with the corresponding Ritz vectors in $Q_{n} u$. In this formulation, it is understood that $m$ is too large to compute the fully reduced Hessenberg form $H$ in Eq. (3.163), and that only a portion of the eigenvalue spectrum $k$ is desired. So, it computes the $n \times n$ matrix $H_{n}$, where the first $n$ columns of $Q$ is the $m \times n$ matrix $Q_{n}$. The Arnoldi iteration has an inner and outer loop and proceeds as follows:

- Set an arbitrary initial vector $b$ and normalize it $q_{1}=b /\|b\|$.

- Set $i=1$. 
- Apply the matrix $A$ to the vector $v=A q_{i}$.

- Compute the $i$ th column of the $H_{n}$ matrix as follows.

- Set $j=1$.

- Compute the $j$ th element of the $i$ th column $h_{j i}=q_{j}^{*} v$

- Subtract this component from the vector $v=v-h_{j i} q_{j}$.

- Set $j=j+1$, and repeat the last two steps until $j=i$.

- Compute the sub-diagonal element of the $i$ th column $h_{i+1, i}=\|v\|$.

- Renormalize the vector for the next column $q_{i+1}=v / h_{i+1, i}$.

- Set $i=i+1$, and repeat starting from step 3 .

In this procedure, the matrix $A$ only appears as the application to the vector $A q_{i}$. To compute the eigenvalues of $A$, at each step $i$ or at occasional steps, perform a direct factorization of the $H_{i}$ matrix to Schur form using techniques discussed in $\S 3.4 .1$ to obtain the Ritz values and vectors. Some of these values converge rapidly, and upon convergence to machine precision, it is assumed that these are the eigenvalues of $A$. This technique generally converges to the extreme eigenvalues of $A$ near the edge of the spectrum, with an increased geometric convergence occurring for relatively isolated eigenvalues [12].

Specifically, ARPACK uses the Implicit Restarted Arnoldi Method (IRAM) to compute eigenvalues. If the Arnoldi iteration is left to run where $n$ becomes large, storage requirements for the vector set $Q_{n}$ increases as well as the computational demand associated with calculating the $H_{n}$ matrix. Implicit restarting addresses these issues, with an additional benefit in that it allows for targeting the $k$ desired eigenvalues of $A$. The basic idea is that after an initial predetermined number of steps $n>k+p$, where $p \geq k$, the Arnoldi process restarts with a new initial vector computed to enhance the components of the desired eigenvalues and suppress the components of the next undesired eigenvalues [16]. Then, $p$ additional steps of the Arnoldi process is able to obtain the $n \times n$ Hessenberg reduction. Unfortunately, there is no way to determine the optimal value for $n$, but there are two factors to consider: as $n$ increases, fewer Arnoldi iterations are necessary to obtain the $k$ desired eigenvalues; with larger $n$, the computational time to reduce the matrix to Schur form increases.

\section{The Fixed-Source Problem}

As implemented in ARPACK, the IRAM returns either the smallest or largest magnitude eigenvalues in the spectrum, or those eigenvalues closest to an applied shift. In practice, the smallest magnitude $\alpha$ eigenvalues are of interest because they are the 
longest-lived. Because it is more convenient to find the largest magnitude eigenvalues with IRAM, a shift of the $\alpha$-eigenvalue spectrum is sometimes useful in increasing efficiency of IRAM. This is performed by finding the eigenvalues $\lambda$ of $A^{-1}$ instead of $A$, where the eigenvalues are related by $\lambda=\alpha^{-1}$. While this is a simple theoretical transformation, difficulties arise in practice due to needing to apply the inverse to a vector in the Arnoldi iteration $A^{-1} q_{i}$. This application takes the form of the fixed-source problem

$$
A v=q_{i}
$$

Several methods and preconditioners are available to efficiently solve such problems. Because these methods are not implemented for this thesis, they are left from the discussion.

\section{References}

[1] W. J. Stewart, Probability, Markov Chains, Queues, and Simulation. Princeton, New Jersey: Princeton University Press, 2009.

[2] G. I. Bell and S. Glasstone, Nuclear Reactor Theory. New York: Van Nostrand Reinhold Company, 1970.

[3] E. Parzen, Stochastic Processes. California: Holden-Day, 1962.

[4] S. M. Ross, Stochastic Processes. New York: John Wiley \& Sons, 1996.

[5] F. B. Brown, S. E. Carney, B. C. Kiedrowski, and W. R. Martin, "Fission Matrix Capability for MCNP, Part I - Theory," Tech. Rep. LA-UR-13-20429, Los Alamos National Laboratory, 2013.

[6] S. E. Carney, F. B. Brown, B. C. Kiedrowski, and W. R. Martin, "Fission Matrix Capability for MCNP, Part II - Applications," Tech. Rep. LA-UR-13-20454, Los Alamos National Laboratory, 2013.

[7] M. B. Chadwick, P. Obložinký, M. Herman, et al., "ENDF/B-VII.0: Next Generation Evaluated Nuclear Data Library for Nuclear Science and Technology,", Nucl. Data Sheets. 107, pp. 2931-3060, 2006.

[8] M. B. Chadwick, P. Obložinký, M. Herman, et al., "ENDF/B-VII.1: Nuclear Data For Science and Technology: Cross Sections, Covariance, Fission Product Yields and Decay Data,", Nucl. Data Sheets. 112, pp. 2887-2996, 2011.

[9] J. J. Duderstadt and W. R. Martin, Transport Theory. New York: John Wiley \& Sons, 1979.

[10] K. Banerjee, Kernel Density Estimator Methods for Monte Carlo Radiaton Transport. PhD thesis, University of Michigan, 2010.

[11] B. Bradie, A Friendly Introduction to Numerical Analysis. Upper Saddle River, New Jersey: Pearson Prentice Hall, 2006.

[12] L. N. Trefethen and D. B. Bau III, Numerical Linear Algebra. Philadelphia, Pennsylvania: Society for Industrial and Applied Mathematics, 1997.

[13] MATLAB, version 7.10.0. Natick, Massachusetts: The Mathworks Inc., 2010. 
[14] E. Anderson, Z. Bai, C. Bischof, S. Balackford, J. Demmel, J. Dongarra, J. Du Croz, A. Greenbaum, S. Hammarling, A. McKenney, and D. Sorensen, LAPACK Users' Guide. Philadelphia, Pennsylvania: Society for Industrial and Applied Mathematics, Third ed., 1999.

[15] P. Arbenz, "Solving Large Scale Eigenvalue Problems." Swiss Federal Institute of Technology Zurich (ETH Zürich), Lecture Notes, Spring, 2012.

[16] R. B. Lehoucq, D. C. Sorensen, and C. Yang, ARPACK Users' Guide: Solution of Large Scale Eigenvalue Problems with Implicitly Restarted Arnoldi Methods. Philadelphia, Pennsylvania: Society for Industrial and Applied Mathematics, 1998. 


\section{CHAPTER 4}

\section{Eigenvalues and Eigenfunctions of Infinite Media}

This chapter discusses the implementation of the TRMM to infinite, i.e., 0-D, media, where the TRM characterizes neutrons moving through the energy phase space. A research Monte Carlo code calculates transition rates and uses linear algebra routines to calculate the eigenpairs of the forward and adjoint matrices. Multigroup problems provide analytic $\alpha$ eigenvalues for verification of the TRMM. For more complex continuous-energy problems, parametric studies show the effect of the discretization of the energy phase space on the calculated $\alpha$ eigenvalues. This includes a discussion on convergence observations of the $\alpha$-eigenvalue spectrum and its effect on expanded time-dependent solutions. A TDMC solution provides the actual flux time behavior for verification of the TRMM eigenfunction expansion and a discussion on the quantification of the residual term $\zeta(E, t)$ ends the chapter.

\subsection{Code (TORTE)}

In the first implementation of the TRMM, a collection of MATLAB [1] functions generates neutron histories in a continuous-energy infinite medium, calculates transition rates, builds the TRM, determines eigenvalues, calculates the eigenfunction expansion, and generates plots and movies. This takes advantage of the convenient linear algebra and visualization tools in MATLAB. The multigroup problems in this chapter use this code version. For continuous-energy problems, computational time becomes more of an issue: the code is rewritten in FORTRAN90 [2], using the LAPACK [3] linear algebra package to calculate eigenvalues and gnuplot [4] scripts for visualization. The remaining problems in this chapter use this code version. In either case, this code is named for its purpose: To Obtain Real Time Eigenvalues (TORTE).

TORTE has approximately 20 functions or subroutines, 4 testing scripts, and another 8 post-processing scripts with visualization tools. It tallies removal rates and probabilities for the TRM during a $k$-eigenvalue power iteration, obtaining $k^{C}$ using 
the collision estimator in Eq. (2.66). The use of this estimator creates a correlation between the calculated $k$ and $\alpha$ eigenvalues: events determine the elements of the TRM, and the only type of event that occurs in an infinite medium is a collision. It handles multigroup media with user-specified cross sections or continuous-energy media using ACE-formatted cross sections. For the continuous-energy physics, TORTE considers elastic scattering isotropic and does not model inelastic scattering for fast neutrons. For thermal neutrons, it uses the free-gas model and continuous-S $(\alpha, \beta)$ scattering tables for graphite. It samples both delayed and prompt fission spectra from the cross section files using ENDF Law 4 [5]. TORTE handles common reac-

tor materials such as carbon, hydrogen, oxygen, graphite, ${ }^{235} \mathrm{U}$, and ${ }^{238} \mathrm{U}$. It treats capture and fission as analog, and lacks variance reduction techniques.

To ensure that the TORTE physics engine models collisions and samples from energy distributions properly, the generated results for elastic scattering, free gas scattering, and delayed and prompt fission spectra are benchmarked to expected results [6]. This agreement provides confidence in the continuous-energy physics treatment in TORTE (Figure 4.1).

\subsubsection{Tallying the Transition Rate Matrix}

TORTE tallies the TRM in Eq. (3.48) using $G$ energy intervals and $J$ precursor groups. It normalizes the probabilities, such that

$$
\begin{aligned}
& \sum_{g=1}^{G} \chi_{p g}=1, \\
& \sum_{g=1}^{G} \chi_{j g}=1, \quad \text { for } j=1, \ldots, J \\
& \sum_{j=1}^{J} \beta_{j}=1 .
\end{aligned}
$$

TORTE calculates all matrix elements from tallies made during the Monte Carlo random walk, e.g., it does not use known quantities for decay constants or group delayed neutron fractions directly in the matrix elements. Because of this, known decay constants and delayed fractions are useful as checks on the estimated values. All elements are combinations of removal rates, probabilities of given events, and 
Free gas scatter; $\mathrm{A}=10 ; \mathrm{E}_{\mathrm{in}}=1 \mathrm{k}_{\mathrm{b}} \mathrm{T}(0.0253 \mathrm{eV})$

Prompt fission spectrum
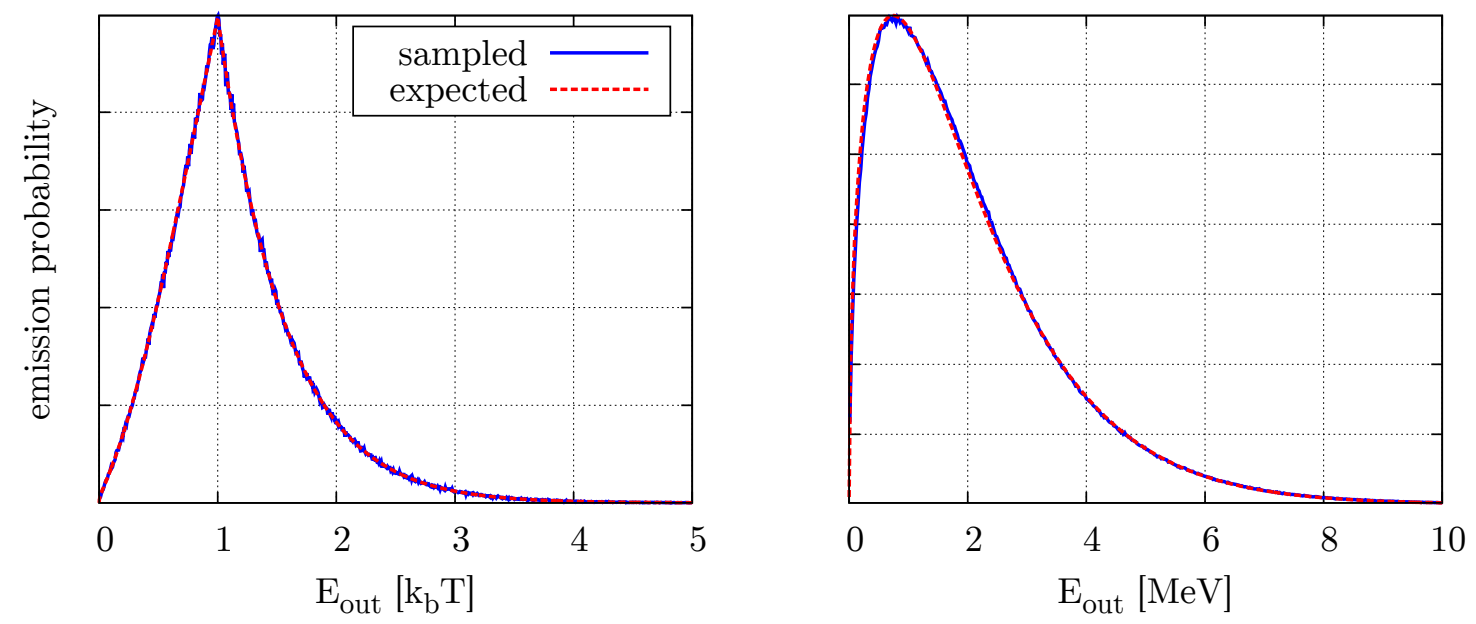

Figure 4.1: The TORTE free-gas scattering outgoing energy spectrum and prompt fission emission spectrum $\chi_{p}(E)$ agree with expected analytic distributions: the piecewise free-gas scattering kernel and Watt fission spectrum for thermal neutron-induced fission.

additional multipliers, such as

$$
\begin{aligned}
\lambda_{j} & =\tau_{r j}^{-1}=(\text { average decay time from precursor group } j)^{-1}, \\
\left(v \Sigma_{r}\right)_{g} & =\tau_{r g}^{-1}=(\text { average removal time from energy interval } g)^{-1}, \\
\left(v \Sigma_{f}\right)_{g} & =\left(v \Sigma_{r}\right)_{g} \frac{\Sigma_{f g}}{\Sigma_{r g}}=\tau_{r g}^{-1}\left(\frac{\text { fissions in energy interval } g}{\text { removals from energy interval } g}\right), \\
\left(v_{g} \Sigma_{s g g^{\prime}}\right) & =\left(v \Sigma_{r}\right)_{g} \frac{\Sigma_{s g g^{\prime}}}{\Sigma_{r g}}=\tau_{r g}^{-1}\left(\frac{\text { scatters from energy interval } g \rightarrow g^{\prime}}{\text { removals from energy interval } g}\right) .
\end{aligned}
$$

The number and size of the energy intervals is arbitrarily set before the first Monte Carlo cycle. For the continuous-energy cases, the discretized energy phase space consists of equal-lethargy intervals.

\subsubsection{Eigenvalues and Eigenfunctions}

TORTE calculates up to $G+J \alpha$ eigenvalues and eigenfunctions for the forward and adjoint matrix equations. The eigenfunctions are vectors of length $G+J$, where the first $G$ entries correspond to the integrated neutron flux in the $g$ th energy interval $\psi_{g}$, and the last $J$ entries correspond to the $j$ th group delayed precursor concentration $C_{j}$,

$$
\left[\begin{array}{ll}
\psi(E) & C
\end{array}\right]=\left[\begin{array}{llllllll}
\psi_{1} & \psi_{2} & \cdots & \psi_{G} & C_{1} & \cdots & C_{J-1} & C_{J}
\end{array}\right] .
$$


In cases where the main interest is in the flux energy spectrum, the eigenfunction expansions exclude the last $J$ entries of the eigenfunction in Eq. (4.8). With this simplified infinite medium, the coefficients in Eq. (2.92) are inner products of the adjoint eigenvectors and desired initial source. Then, the eigenfunction expansion for the neutron flux becomes

$$
\psi(E, t)=\sum_{i=0}^{M} \frac{\psi_{i}^{\dagger}(E) \cdot Q_{0}(E)}{\gamma_{i}} \psi_{i}(E) \exp \left(\alpha_{i} t\right)
$$

where $M$ identifies the desired number of modes from $0 \leq M \leq G+J-1$ used to approximate the flux solution. As $M$ increases, this expansion captures increasingly shorter time dependence. For the infinite medium case, the sum of a pair of complex eigenvalues $\alpha_{i}$ and $\alpha_{j}=\bar{\alpha}_{i}$ from Eq. (2.101) simplifies to

$$
\psi_{i+j}(E, t)=2\left[D_{1} \cos \left(\left|\operatorname{Im}\left(\alpha_{i}\right)\right| t\right)-D_{2} \sin \left(\left|\operatorname{Im}\left(\alpha_{i}\right)\right| t\right)\right] \exp \left(\operatorname{Re}\left(\alpha_{i}\right) t\right)
$$

where the vectors

$$
\begin{aligned}
& D_{1}=\operatorname{Re}\left(A_{i}\right) \operatorname{Re}\left(\psi_{i}\right)-\operatorname{Im}\left(A_{i}\right) \operatorname{Im}\left(\psi_{i}\right), \\
& D_{2}=\operatorname{Re}\left(A_{i}\right) \operatorname{Im}\left(\psi_{i}\right)+\operatorname{Im}\left(A_{i}\right) \operatorname{Re}\left(\psi_{i}\right) .
\end{aligned}
$$

This equation describes an under-damped, all-real solution with damped frequency $\omega_{d}=\left|\operatorname{Im}\left(\alpha_{i}\right)\right|$. An approximation for the number of oscillations that occur before damping is the ratio $\left|\omega_{d}: \operatorname{Re}\left(\alpha_{i}\right)\right|$.

\subsection{Multigroup Verification}

Due to the simplified TORTE physics engine, results do not match MCNP5 [5] or other transport codes. Thus, calculated results are compared to analytic solutions. Five multiplying, multigroup problems test the ability of TORTE to calculate the appropriate $k$ eigenvalue, approximate the TRM, and obtain $\alpha$ eigenvalues. The first two are three-group problems with and without delayed neutron emission. The last three are variations on a simplified 81-group problem with prompt fission only. These variations show the effect of the spectrum on the eigenfunction expansion and the effect of some nuclear parameters on the calculated spectrum. 


\subsubsection{Few-Group Media}

Problem 1: This three-group medium (Table 4.1) has fissions in energy group $g=$ 3 emitting $\bar{\nu}=2.5$ neutrons in energy group $g=1$. There is no upscatter and downscatter is only allowed into the next group.

The analytic $k$ eigenvalue is

$$
k=\frac{\bar{\nu} \Sigma_{f} \Sigma_{s 12} \Sigma_{s 23}}{\Sigma_{r 1} \Sigma_{r 2} \Sigma_{r 3}} \approx 0.83333
$$

where $\Sigma_{r g}=\Sigma_{\gamma g}+\Sigma_{f g}+\Sigma_{s g, g+1}$. The analytic TRM is

$$
\left[\begin{array}{ccc}
-v_{1} \Sigma_{r 1} & v_{1} \Sigma_{s 12} & 0 \\
0 & -v_{2} \Sigma_{r 2} & v_{2} \Sigma_{s 23} \\
v_{3} \chi \bar{\nu} \Sigma_{f} & 0 & -v_{3} \Sigma_{r 3}
\end{array}\right]=\left[\begin{array}{ccc}
-24 & 20 & 0 \\
0 & -10 & 8 \\
5 & 0 & -4
\end{array}\right]
$$

and the fundamental $\alpha$ eigenvalue is $-0.44534 \mathrm{~s}^{-1}$. With $1 \times 10^{6}$ histories, TORTE calculates the $k$ eigenvalue $0.83377 \pm 0.00059$ and the error in the calculated TRM is

$$
\left[\begin{array}{ccc}
0.002 & 0.013 & - \\
- & 0.036 & 0.077 \\
-0.022 & - & 0.025
\end{array}\right] \%
$$

This matrix represents the element-by-element percentage difference between the analytical TRM and that calculated by TORTE. The analytic $k$ eigenvalue is within one standard deviation of the calculated $k$ eigenvalue and the entries of the calculated TRM are within $0.1 \%$ of those of the analytic TRM. The calculated fundamental $\alpha$ eigenvalue is within one thousandth of a percent of the analytic fundamental $\alpha$ eigenvalue.

Table 4.1: Problem 1 nuclear data. Cross sections are in $\mathrm{cm}^{-1}$.

\begin{tabular}{cccccc}
\hline$g$ & $\Sigma_{\gamma g}$ & $\Sigma_{f}$ & $\Sigma_{s g, g+1}$ & $\chi$ & $v_{g}[\mathrm{~cm} / \mathrm{s}]$ \\
\hline 1 & 1.0 & 0 & 5.0 & 1.0 & 4.0 \\
2 & 1.0 & 0 & 4.0 & 0 & 2.0 \\
3 & 2.0 & 2.0 & 0 & 0 & 1.0 \\
\hline
\end{tabular}

Problem 2: This three-group medium uses two precursor groups (Table 4.2) to model the emission of $\bar{\nu}_{d}=0.5$ delayed neutrons per fission. Other nuclear data is the same as Problem 1. To preserve the $k$ eigenvalue from Eq. (4.13), the average total neutrons emitted per fission $\bar{\nu}_{t}$ matches that of Problem 1. All precursors emit 
Table 4.2: Problem 2 precursor data.

\begin{tabular}{ccc}
\hline$j$ & $\lambda_{j}\left[\mathrm{~s}^{-1}\right]$ & $\beta_{j}$ \\
\hline 1 & 3.0 & 0.25 \\
2 & 1.0 & 0.75 \\
\hline
\end{tabular}

neutrons into energy group $g=1$.

The analytic $k$ eigenvalue is the same as in Problem 1 and the analytic TRM is

$$
\begin{gathered}
{\left[\begin{array}{ccccc}
-v_{1} \Sigma_{r 1} & v_{1} \Sigma_{s 12} & 0 & 0 & 0 \\
0 & -v_{2} \Sigma_{r 2} & v_{2} \Sigma_{s 23} & 0 & 0 \\
v_{3} \chi_{p} \bar{\nu}_{p} \Sigma_{f} & 0 & -v_{3} \Sigma_{r 3} & v_{3} \beta_{1} \bar{\nu}_{d} \Sigma_{f} & v_{3} \beta_{2} \bar{\nu}_{d} \Sigma_{f} \\
\chi_{11} \lambda_{1} & 0 & 0 & -\lambda_{1} & 0 \\
\chi_{21} \lambda_{2} & 0 & 0 & 0 & -\lambda_{2}
\end{array}\right]=} \\
{\left[\begin{array}{ccccc}
-24 & 20 & 0 & 0 & 0 \\
0 & -10 & 8 & 0 & 0 \\
4 & 0 & -4 & 0.25 & 0.75 \\
3 & 0 & 0 & -3 & 0 \\
1 & 0 & 0 & 0 & -1
\end{array}\right]}
\end{gathered}
$$

where $\bar{\nu}_{t}=\bar{\nu}_{p}+\bar{\nu}_{d}$, and the analytic fundamental $\alpha$ eigenvalue is $-0.29137 \mathrm{~s}^{-1}$. With $1 \times 10^{6}$ histories, TORTE calculates the $k$ eigenvalue $0.83351 \pm 0.00059$, and the error in the calculated TRM is

$$
\left[\begin{array}{ccccc}
-0.158 & -0.121 & - & - & - \\
- & -0.106 & -0.122 & - & - \\
0.063 & - & -0.073 & -0.052 & 0.101 \\
0.465 & - & - & 0.465 & - \\
-0.374 & - & - & - & -0.374
\end{array}\right] \%
$$

Again, the calculated $k$ eigenvalue and TRM are converging to analytic solutions. The entries of the TRM corresponding to delayed neutrons show worse agreement than the rest of the matrix, even with $\bar{\nu}_{d}$ higher than in a realistic problem. This is due to the sampling of the decay constants $\lambda_{i}$, and the low probability of delayed fission relative to prompt fission. The calculated fundamental $\alpha$ eigenvalue is $-0.29414 \mathrm{~s}^{-1}$, and is within $1 \%$ of the analytic fundamental $\alpha$ eigenvalue.

These two problems verify the ability of TORTE to accurately calculate the $k$ eigenvalue, $\alpha$ eigenvalue, and the TRM for multigroup problems. 


\subsubsection{1-Group Media}

Problem 3: This is the base-case $G=81$ group medium (Table 4.3) for examining features in the $\alpha$-eigenvalue spectrum. Neutrons only downscatter to the next energy group. Prompt fissions in energy group $g=81$ emit an average $\bar{\nu}=2.5$ neutrons per fission into energy group $g=1$. There are no delayed neutron precursors.

The capture cross sections $\Sigma_{\gamma g}$, neutron speeds $v_{g}$, and removal cross sections $\Sigma_{r g}$, are the same for all groups so that this very unphysical medium yields a simple, closed-form solution for the $k$ eigenvalue and $\alpha$-eigenvalue spectrum. The analytic $k$ eigenvalue is

$$
k=\frac{\bar{\nu} \Sigma_{f}\left(\Sigma_{s g, g+1}\right)^{G-1}}{\left(\Sigma_{r g}\right)^{G}}=1.11663
$$

and is within two standard deviations of the TORTE $k$ eigenvalue, $1.1170 \pm$ 0.0012. Algebraic manipulation of the multigroup equations [7] yields the analytic $\alpha$-eigenvalue spectrum

$$
\frac{\alpha_{n}}{v}=-\Sigma_{\gamma g}+\Sigma_{s g, g+1}\left[\bar{\nu}^{-1} \exp \left(2 \pi i n G^{-1}\right)-1\right], \quad \text { for } \quad n=0, \ldots, G-1
$$

The analytic $\alpha$ eigenvalues align along a circle in the complex plane centered on the real axis at $\operatorname{Re}(\alpha)=-\Sigma_{\gamma g}-\Sigma_{s g, g+1}$ with a radius of $r=\bar{\nu}^{G^{-1}} \Sigma_{s g, g+1}$, where the fundamental $\alpha$ eigenvalue is $0.13765 \mathrm{~s}^{-1}$. As the number of groups increases, the $\alpha$ eigenvalue spectrum shrinks because the $k$ eigenvalue changes; however, if the group cross sections are adjusted such that the $k$ eigenvalue remains constant, the additional calculated $\alpha$ eigenvalues fill in along the existing circle. The calculated $\alpha$-eigenvalue spectrum matches this analytic solution (Figure 4.2), where the eigenvalues with the smallest real parts show the best agreement.

For this case, there is only one all-real eigenvalue, which TORTE calculates as $0.13839 \mathrm{~s}^{-1}$ : this is within $1 \%$ of the analytic fundamental $\alpha$ eigenvalue. All higher eigenfunctions are complex and contribute some oscillatory time dependence to the expanded flux solution. There are oscillations that persist for long times, shown by the higher $\alpha$ eigenvalues that have a real part close to that of the fundamental eigenvalue.

Table 4.3: Problem 3 nuclear data. Cross sections are in $\mathrm{cm}^{-1}$.

\begin{tabular}{cccccc}
\hline$g$ & $\Sigma_{\gamma g}$ & $\Sigma_{f}$ & $\Sigma_{s g, g+1}$ & $\chi$ & $v_{g}[\mathrm{~cm} / \mathrm{s}]$ \\
\hline 1 & 1.0 & 0 & 100.0 & 1.0 & 1.0 \\
$2-80$ & 1.0 & 0 & 100.0 & 0 & 1.0 \\
81 & 1.0 & 100.0 & 0 & 0 & 1.0 \\
\hline
\end{tabular}




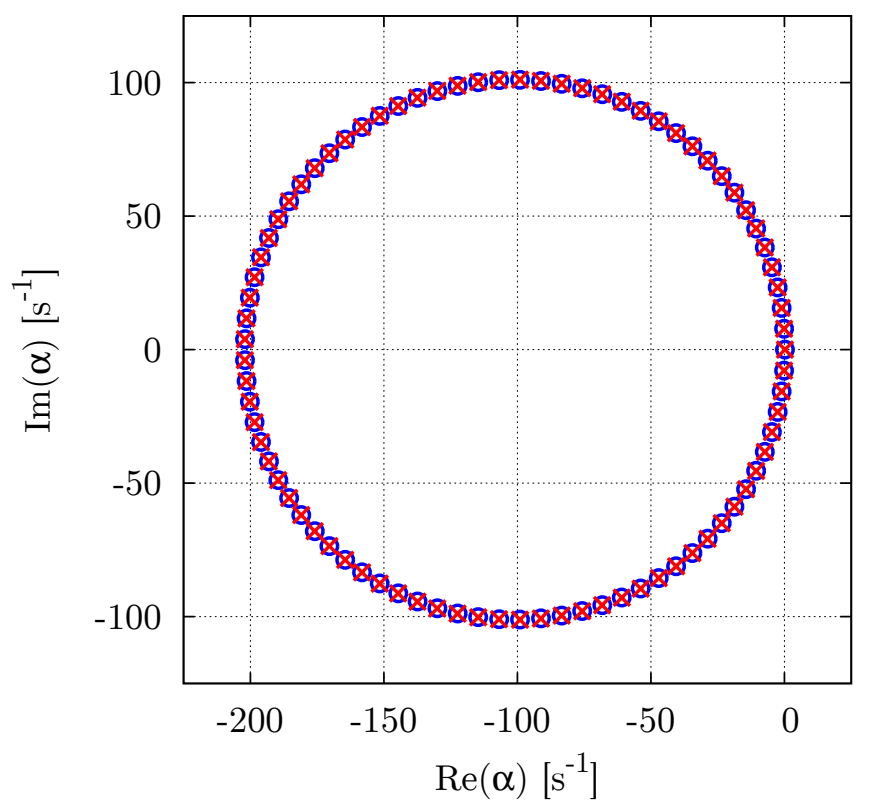

calculated $\odot \quad$ analytic $\times$

Figure 4.2: The $81 \alpha$ eigenvalues of Problem 3 form a circle in the complex plane. The TORTE-calculated spectrum using $1 \times 10^{6}$ histories converges to the equally-spaced eigenvalues of the analytic solution.

These $\alpha$ eigenvalues have a large imaginary part, such that the ratio $|\operatorname{Im}(\alpha): \operatorname{Re}(\alpha)|$ is high. This causes a finite number of oscillations before the kinetic mode decays: this is very unphysical. The eigenvalues with the largest imaginary parts correspond to the fastest oscillating kinetic modes.

The time-dependent flux solution from the eigenfunction expansion using an initial pulse source (Figure 4.3) shows the effect of some of the calculated eigenvalues. This provides for more understanding of the spectrum. At $t=0.10 \mathrm{~s}$, neutrons from the monoenergetic source scatter out of energy group $g=1$ at different times, resulting in a small flux packet that begins to downscatter. At $t=0.70 \mathrm{~s}$, the flux packet continues to widen and decrease due to the different rates at which neutrons downscatter and the small capture cross section. When neutrons reach energy group $g=81$, they induce fission and emit several neutrons in energy group $g=1$. At $t=1.60 \mathrm{~s}$, the flux packet widens to the point where it combines with the neutrons fissioning into energy group $g=1$. At $t=6.00 \mathrm{~s}$, individual flux packets disappear as the flux approaches and follows the rising fundamental mode.

As $t \rightarrow \infty$, the higher modes decay and the expanded flux solution approaches the fundamental mode. This happens at $t \approx 20 \mathrm{~s}$ in this medium, which is long 

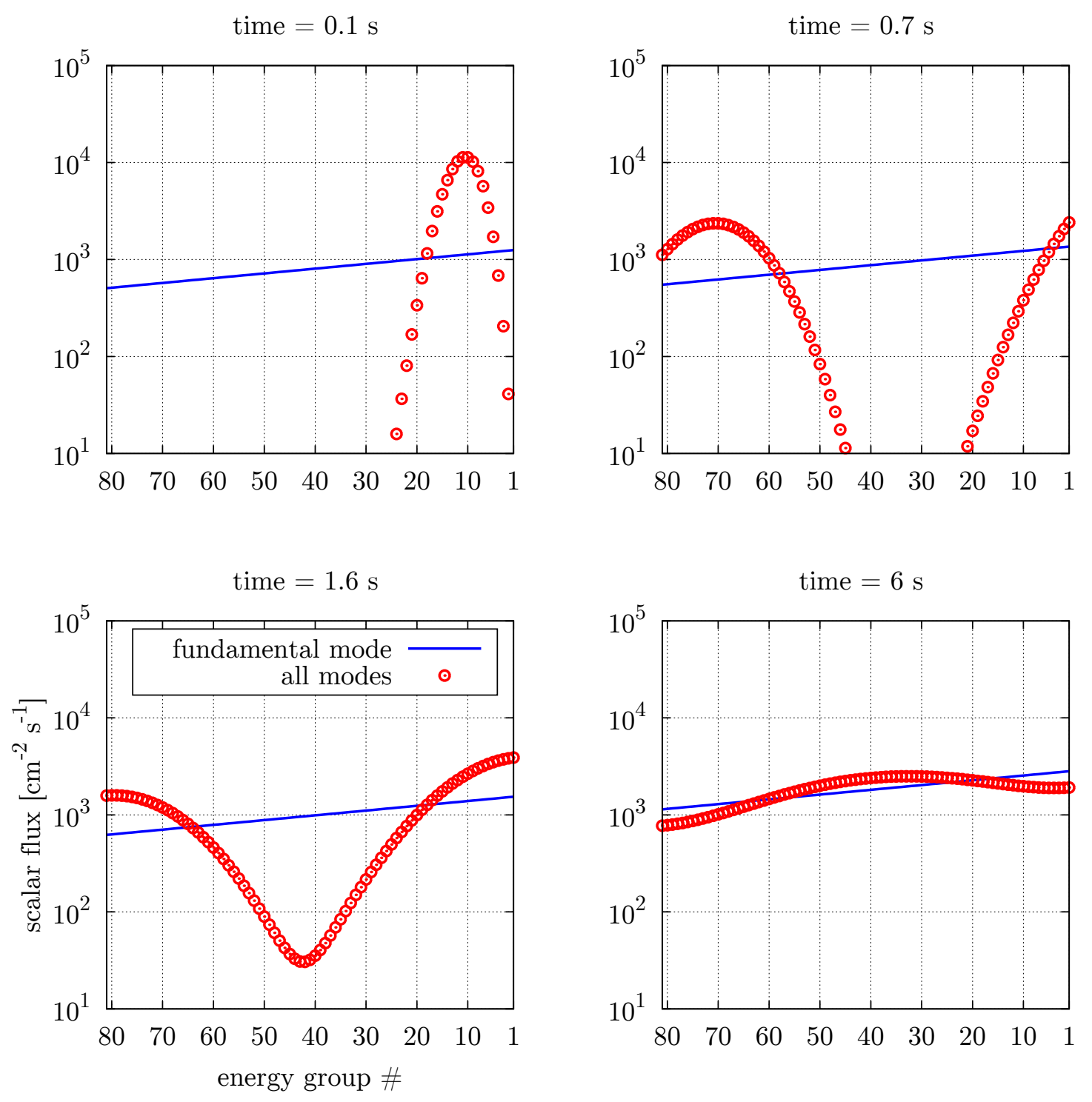

Figure 4.3: Snapshots of the Problem 3 eigenfunction-expanded flux solution demonstrate the difference between the full solution using all 81 modes and the solution using only the fundamental mode. The initial source is a monoenergetic pulse at $t=0$ in energy group $g=1$, with source strength $Q_{0}(1)=5 \times 10^{4} \mathrm{~cm}^{-3} \cdot \mathrm{s}^{-1}$.

relative to the lifetime of the prompt modes. If this medium had delayed precursors, its delayed modes would persist for longer times. Note that the fundamental mode increases considerably as the higher modes decay. All coefficients and eigenfunctions are complex for $i=1, \ldots, G-1$.

Problem 4: This variant of the 81-group medium changes the speeds to be group-dependent, where $v_{g}=82-g$. The $k$ eigenvalue is the same as the base-case, but the $\alpha$-eigenvalue spectrum loses its closed-form analytic solution: the analytic 
TRM provides the $\alpha$ eigenvalues. The analytic fundamental $\alpha$ eigenvalue is 2.2464 $\mathrm{s}^{-1}$. TORTE calculates the $k$ eigenvalue $1.1139 \pm 0.0012$, within three standard deviations of the analytic $k$ eigenvalue. The calculated fundamental $\alpha$ eigenvalue is $2.2008 \mathrm{~s}^{-1}$, and is within $2.5 \%$ of the analytic fundamental $\alpha$ eigenvalue. TORTE has more difficulty converging to the analytic solution in this multiple-speed medium than for the base-case. This is particularly true for the higher complex eigenvalues (Figure 4.4). Also, note that the calculated $k$ eigenvalue and fundamental $\alpha$ eigenvalue are correlated: both calculated quantities under-predict expected values.

With different group speeds, the $\alpha$ eigenvalue spectrum is no longer arranged along a circle, but some elliptical shapes remain. There are two more very negative real eigenvalues and some eigenvalues form lines crossing the real axis. The eigenvalues closest to the fundamental eigenvalue converge faster than higher eigenvalues. The extent of the spectrum increases approximately $25 \times$ along the imaginary axis and $45 \times$ along the real axis. This implies an increase in the rate at which higher modes decay, and an increase in the rate of oscillations. This is due to neutrons moving with a greater speed through each energy group and downscattering faster. Higher modes decay rapidly, and the expanded flux solution using all modes approaches the fundamental mode in less time than in the base-case.

Problem 5: This variant of the 81-group medium allows downscattering from

Problem 4

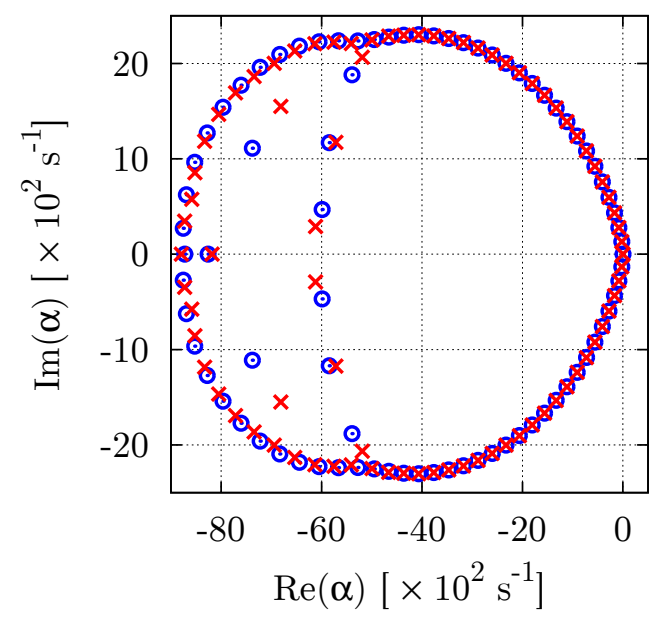

calculated $\odot$
Problem 5

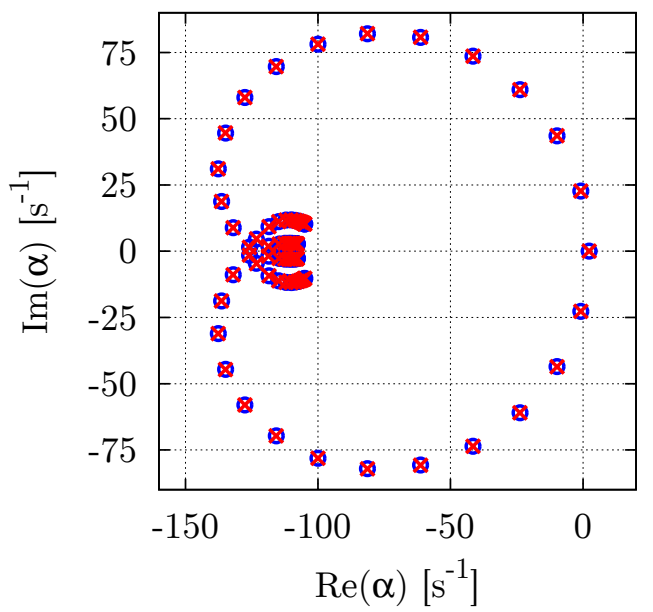

analytic $x$

Figure 4.4: The calculated 81-group variant $\alpha$-eigenvalue spectra with different group speeds (Problem 4) and downscattering over up to 5 groups (Problem 5) converge to analytic solutions at different rates. TORTE calculates both spectra using $1 \times 10^{6}$ histories. 
energy group $g \rightarrow g^{\prime}$ over several groups with equal probability where $g+1 \leq g^{\prime} \leq$ $g+5$. For the the last 5 groups, the downscattering probability is equally distributed to the remaining groups, where $g+1 \leq g^{\prime} \leq G$. The total downscattering cross section is the same, but because the multigroup equations change, the $k$ eigenvalue changes, as does the $\alpha$-eigenvalue spectrum. The analytic fundamental $\alpha$ eigenvalue is $2.2914 \mathrm{~s}^{-1}$. TORTE calculates the $k$ eigenvalue $1.7676 \pm 0.0464$ and the fundamental $\alpha$ eigenvalue $2.2937 \mathrm{~s}^{-1}$.

The calculated $\alpha$-eigenvalue spectrum shows much better agreement to the analytic spectrum than in the multiple-speed medium (Figure 4.4). The spectrum has a cluster of $\alpha$ eigenvalues close to the real axis where $\operatorname{Re}(\alpha)<-100 \mathrm{~s}^{-1}$. These correspond to higher modes that oscillate slowly but decay quickly. The spectrum is skewed such that more eigenvalues have larger negative real parts. This is due to neutrons being able to downscatter quickly by skipping several energy groups, even though the speeds are the same as in the base-case. As with the multiple-speed medium, more higher modes decay quickly, but the extent of the spectrum is very similar to that in the base-case. There are still oscillatory modes that persist for longer times, and the expanded flux solution using all modes approaches the fundamental mode at a time similar to the base-case.

Problems 3, 4, and 5 have simplified $\alpha$-eigenvalue spectra that show the effect of nuclear parameters on the calculated spectrum and the eigenfunction expansion. Some features in these multigroup spectra are present in the more complex spectrum of a continuous-energy medium.

\subsection{Continuous-Energy Media}

Three unique, continuous-energy problems demonstrate the ability of the TRMM to describe neutron behavior in a realistic energy phase space. For all problems, TORTE distributes $G-1$ energy intervals equally in lethargy space between $E_{G}=0.001 \mathrm{eV}$ and $E_{1}=20 \mathrm{MeV}$, where the floor of the $G$ th energy interval is the minimum energy $E_{G+1}=E_{\min }=1 \times 10^{-5} \mathrm{eV}$. The total number of energy intervals $G$ depends on the problem and run, identified by number and letter, respectively. There are $J=6$ precursor groups. Inputs to TORTE are the important nuclear data for each problem: the number densities $N_{d}$, atomic ratios, and atomic percent ${ }^{235} \mathrm{U}$ enrichment (Table 4.4). While the $k$-eigenvalue depends on the atomic ratios, the $\alpha$ eigenvalues are very sensitive to number density in these homogeneous problems: selected values reflect realistic densities. The remaining uranium consists only of ${ }^{238} \mathrm{U}$. All continuous-energy 
Table 4.4: Nuclear data for the continuous-energy problems.

\begin{tabular}{ccccccc}
\hline \multirow{2}{*}{ Problem } & \multirow{2}{*}{$N_{d}\left[\mathrm{barn}^{-1} \cdot \mathrm{cm}^{-1}\right]$} & \multicolumn{6}{c}{ Number Ratios } & \multirow{2}{*}{${ }^{235} \mathrm{U}$ [atom \%] } \\
& & $\mathrm{H}$ & $\mathrm{C}$ & $\mathrm{O}$ & $\mathrm{U}$ & \\
\hline $6 \mathrm{~A}-\mathrm{P}$ & 0.072997 & 1 & 0 & 1 & 1 & 100. \\
$7 \mathrm{~A}-\mathrm{D}$ & 0.072997 & 6 & 0 & 5 & 1 & 1.17 \\
$8 \mathrm{~A}-\mathrm{H}$ & 0.078547 & 0 & 80 & 2 & 1 & 4.25 \\
\hline
\end{tabular}

cross sections are at room temperature. Unless otherwise noted, TORTE uses 100 active cycles with $1 \times 10^{6}$ particles per cycle. The rest of the analysis in this chapter uses variations of these three problems.

Problems 6A-P: This medium consists of equal parts of hydrogen, oxygen, and ${ }^{235} \mathrm{U}$. There is little downscattering due to the small amount of hydrogen, so fast fission is the dominant driver of this medium and the energy spectrum is hard. Each of the sixteen different runs, A through $\mathrm{P}$, uses a specified number of energy intervals: run A uses $G=40$, run $\mathrm{P}$ uses $G=640$, and runs B through $\mathrm{O}$ use $G$ in between with increments of 40 . The TORTE-calculated $k$ eigenvalue of this prompt supercritical problem is $2.08057 \pm 12 \mathrm{pcm}$.

Problems 7A-D: This medium consists of hydrogen, oxygen, and LEU in quantities similar to a typical LWR. With the lower enrichment and additional hydrogen, downscattering and thermal fission drive the medium, and resonance absorption becomes more prevalent: the energy spectrum is softer than for the prompt supercritical medium. The TORTE-calculated $k$ eigenvalue is $1.00177 \pm 12 \mathrm{pcm}$. Because there is no leakage, $\beta_{\text {eff }} \approx \beta=0.0065$, making this a delayed supercritical medium.

Problems 8A-H: This medium consists of carbon, oxygen, and LEU in quantities similar to a typical graphite-moderated, gas-cooled reactor. Like the delayed supercritical medium, this is a thermal medium. The TORTE-calculated $k$ eigenvalue of this very subcritical problem is $0.86946 \pm 10 \mathrm{pcm}$.

\subsubsection{Eigenvalue Spectrum}

For each of the three media, TORTE calculates $G$ prompt and $J=6$ delayed $\alpha$ eigenvalues. To test the ability of the TRMM to reproduce the same results, TORTE starts two runs for the prompt supercritical medium with identical energy interval structure and material parameters, but with a different random number seed. Most of the $\alpha$ eigenvalues in the two resulting spectra (Figure 4.5) agree within 1\%, including the two highest eigenvalues calculated, $-5.115 \times 10^{8} \mathrm{~s}^{-1}$ and $-5.152 \times 10^{8} \mathrm{~s}^{-1}$. The fundamental $\alpha$ eigenvalues agree well within $0.01 \%$ at $4.661 \times 10^{7} \mathrm{~s}^{-1}$. Visually, the 


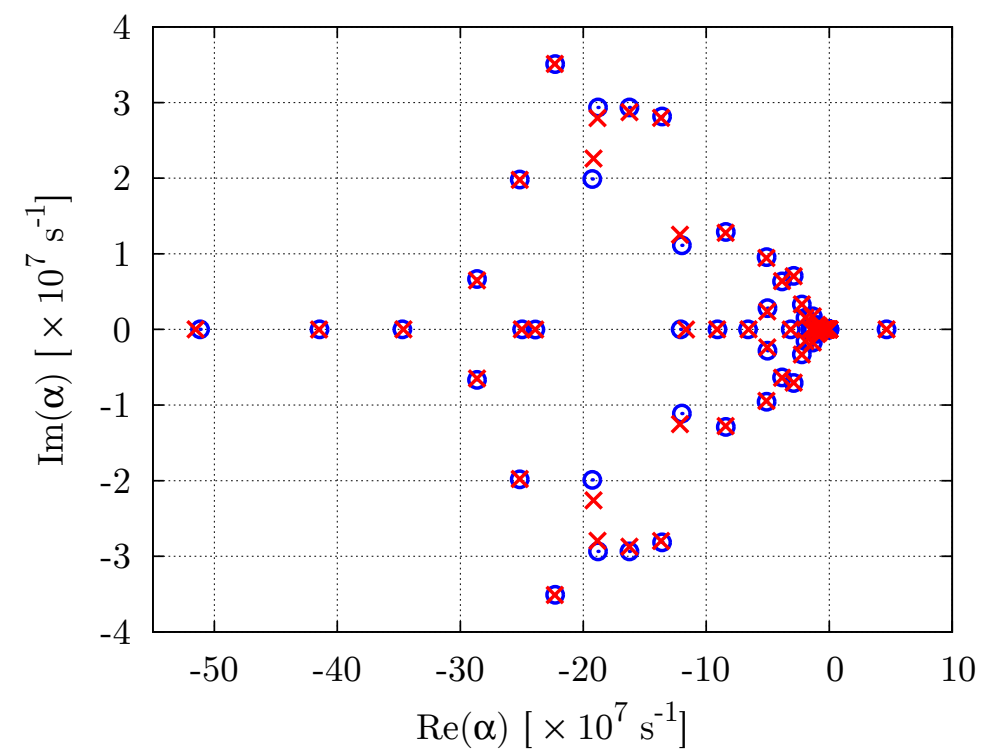

Seed $1 \quad \odot \quad$ Seed $2 \times$

Figure 4.5: The $\alpha$ eigenvalue spectra of the 80 energy interval Problem 6 calculated using two random number seeds only differs for some higher complex eigenvalues. Calculated eigenvalues from both runs are converging to the same spectrum.

worst agreement occurs for a few complex eigenvalues in the interior of the spectrum, e.g., $(-19.27+1.988 i) \times 10^{7} \mathrm{~s}^{-1}$ compared to $(-19.17+2.259 i) \times 10^{7} \mathrm{~s}^{-1}$. Both the real parts and the complex modulus of these two eigenvalues agree within $1 \%$, but the complex part of the eigenvalues show poor agreement: this represents a difference in the oscillation frequency of the corresponding kinetic mode.

With $G=600$ energy intervals, the $\alpha$-eigenvalue spectrum of this prompt supercritical medium (Figure 4.6) has additional features absent from the smaller spectrum. There is one large, positive eigenvalue and all other eigenvalues have negative real parts: all higher kinetic modes decay as $t \rightarrow \infty$. A large number of eigenvalues form a cluster at the right end of the spectrum close to the real axis: oscillations of their corresponding kinetic modes are relatively slow. There are still elliptical and circular shapes within the spectrum, similar to those seen for the 81-group media (Figure 4.4). The magnitude of the scale is different than the 81-group media due to the realistic neutron speeds, but the relative scaling between the real and imaginary axes have more similarities to the multiple-speed medium. The imaginary extent of the spectrum spans approximately a tenth of the real extent: higher kinetic modes corresponding to complex eigenvalues oscillate less than once before decaying. Some eigenvalues begin to trace out short, irregularly-spaced lines. 


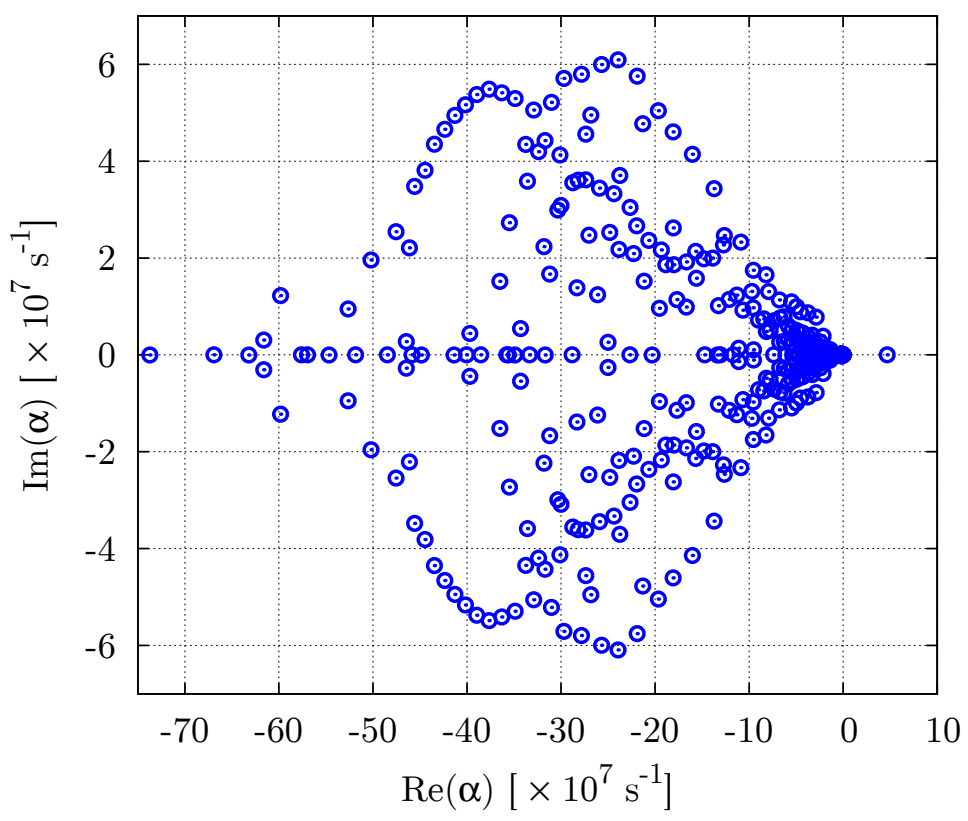

Figure 4.6: The $\alpha$-eigenvalue spectrum of the 600 energy interval Problem 6 has some similar spectral behavior as the 81-group problems, with some eigenvalues aligning along ellipses and curves.

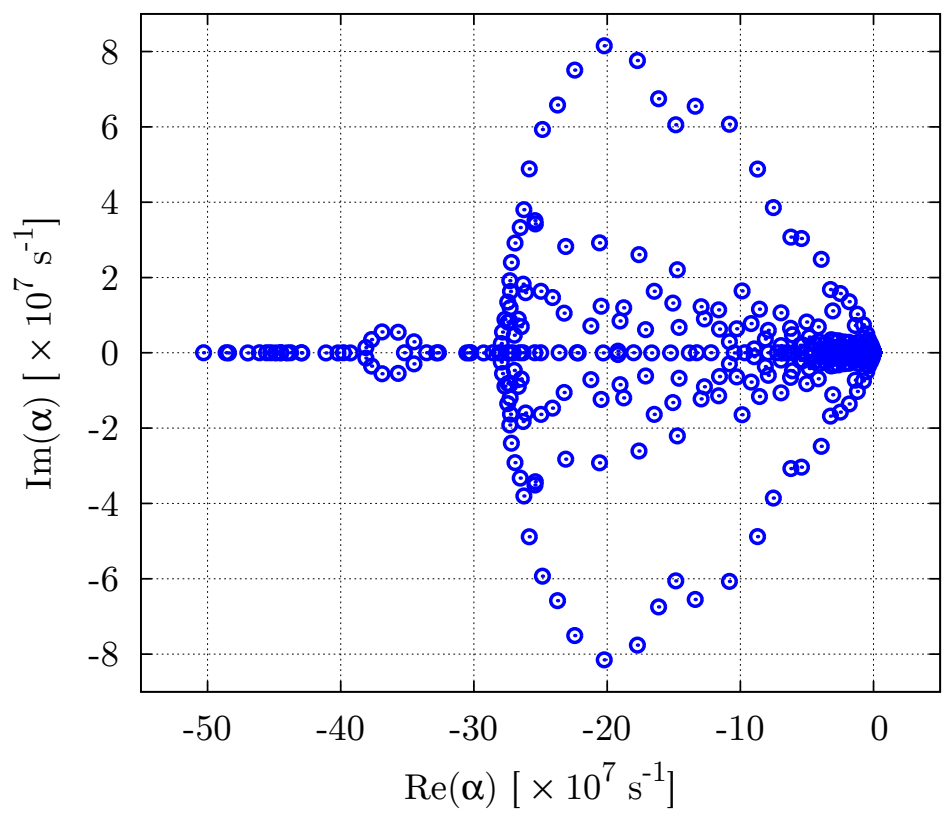

Figure 4.7: The $\alpha$-eigenvalue spectrum of the 640 energy interval Problem 8 is completely negative and has a similar extent as the prompt supercritical problem but with fewer spectral features and more eigenvalues shifted towards the imaginary axis. 
The $\alpha$-eigenvalue spectrum of the thermal, graphite-moderated, subcritical medium (Figure 4.7) has a similar extent and shape as the fast, prompt supercritical spectrum, even though the material compositions are very different. There are fewer complex eigenvalues, and those that exist are closer towards the imaginary axis: the corresponding kinetic modes oscillate more before decaying. While the prompt supercritical medium has many complex eigenvalues located where $\operatorname{Re}(\alpha)<-30 \times 10^{7} \mathrm{~s}^{-1}$, this subcritical medium has very few. All eigenvalues are negative, so the flux decays as $t \rightarrow \infty$, as expected. The prompt fundamental eigenvalue is $-940.1 \mathrm{~s}^{-1}$. The $\alpha-$ eigenvalue spectrum of the delayed supercritical medium is similar to this spectrum (Figure 4.7). The primary difference is that complex eigenvalues in the spectrum of the delayed supercritical medium have very small imaginary parts, $|\operatorname{Im}(\alpha)|<10^{7}$ $\mathrm{s}^{-1}$, for eigenvalues toward the front of the spectrum, where $\operatorname{Re}(\alpha)>-10 \times 10^{7} \mathrm{~s}^{-1}$. Also, the imaginary extent of the spectrum reduces close to $30 \%$.

The features seen in these spectra (Figures 4.6 and 4.7) are solely from prompt $\alpha$ eigenvalues. Due to the extent of the spectra, the six delayed eigenvalues are lost in the cluster close to the imaginary axis. While the fundamental eigenvalue in the prompt supercritical medium is a prompt eigenvalue, those of the delayed supercritical and subcritical media are delayed eigenvalues. For the prompt supercritical and subcritical media, the delayed eigenvalues (Table 4.5) nearly match the precursor decay constants (Table 2.1). In the delayed supercritical medium, the fundamental eigenvalue is positive and on the order of the decay constants, and it shifts the remaining delayed eigenvalues. This medium also has a discrete prompt fundamental eigenvalue of $-78.55 \mathrm{~s}^{-1}$.

Table 4.5: A comparison of calculated delayed $\alpha$ eigenvalues. Units are $\mathrm{s}^{-1}$.

\begin{tabular}{ccccccc}
\hline Run & $\alpha_{d 0}$ & $\alpha_{d 1}$ & $\alpha_{d 2}$ & $\alpha_{d 3}$ & $\alpha_{d 4}$ & $\alpha_{d 5}$ \\
\hline $6 \mathrm{P}$ & -0.01237 & -0.03183 & -0.1096 & -0.3195 & -1.359 & -8.645 \\
$7 \mathrm{D}$ & 0.06957 & -0.01347 & -0.04899 & -0.1575 & -1.138 & -8.044 \\
$8 \mathrm{H}$ & -0.01243 & -0.03160 & -0.1080 & -0.3109 & -1.351 & -8.619 \\
\hline
\end{tabular}

\subsubsection{Energy Intervals}

To investigate the effect of discretizing the energy phase space, TORTE calculates the spectra of the three continuous-energy media with different numbers of energy intervals $G$. The entire $\alpha$-eigenvalue spectrum continues to evolve with increasing $G$ (Figure 4.8). Most importantly, regardless of $G$, all runs for the prompt supercritical medium converge to the same positive fundamental eigenvalue. Some of the higher 


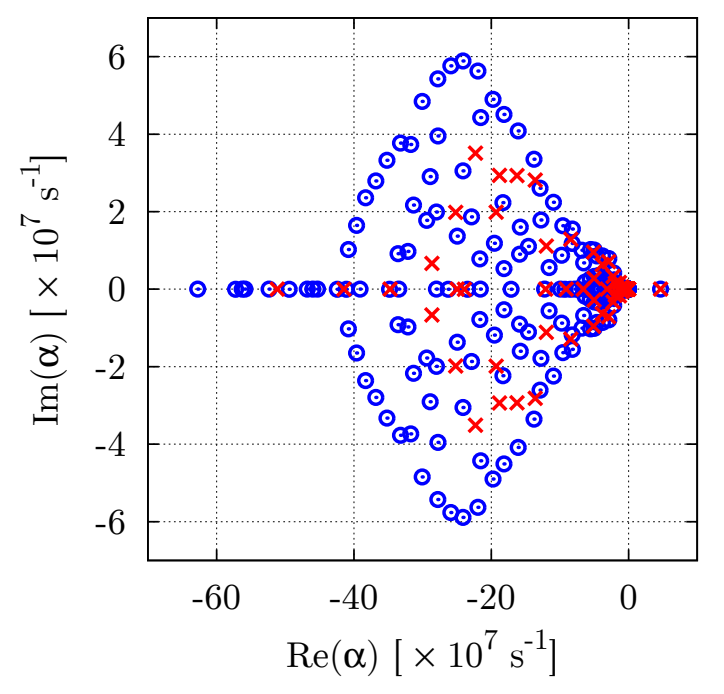

$\mathrm{G}=320(\mathrm{H}) \quad \odot \quad \mathrm{G}=80(\mathrm{~B}) \quad \times$

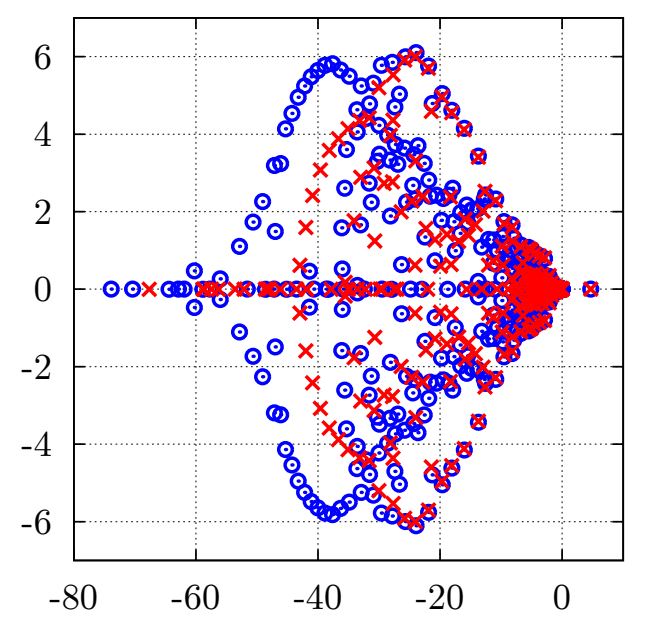

$\mathrm{G}=640(\mathrm{P}) \quad \odot \quad \mathrm{G}=400(\mathrm{~J}) \quad \times$

Figure 4.8: The $\alpha$-eigenvalue spectra of Problem 6 calculated with different numbers of energy intervals agree for the first few eigenvalues but differ on the shape of the higher $\alpha$-eigenvalue spectrum.

$\alpha$ eigenvalues agree, but many do not: the higher eigenvalues of run B fall between those of run $\mathrm{H}$. There is a similar trend seen in the comparison of runs $\mathrm{J}$ and $\mathrm{P}$, although many more eigenvalues seem to agree, especially at the front ridges of the spectra. Increasing $G$ does not increase the extent of the real spectrum in the negative direction, but adds many eigenvalues between existing ones.

Because TORTE uses a finite number of energy intervals and precursor groups, its calculated eigenvalues serve as an approximation to the true $\alpha$-eigenvalue spectrum. This is the reason that many higher eigenvalues of run $B$ fail to match those of run $\mathrm{H}$. These higher run B eigenvalues attempt to capture the nature of the true higher $\alpha$-eigenvalue spectrum, approximating it and falling between the eigenvalues of run $\mathrm{H}$, which serves as a better approximation of the true spectrum. As $G$ increases, TORTE begins to match the true $\alpha$-eigenvalue spectrum, and converges to the true eigenvalues in order, from $i=0,1,2, \ldots, m$ : in the comparison of runs $\mathrm{J}$ and $\mathrm{P}$, eigenvalues approach similar complex values along the front ridges of the spectra.

If TORTE uses very few energy intervals, such as 2 or 3 , it yields an incorrect fundamental eigenvalue because the few calculated eigenvalues attempt to approximate the entire spectrum. This often results in a fundamental eigenvalue that is too low, because the majority of the spectrum exists in the negative real direction. As $G$ increases, the fundamental eigenvalue increases to asymptotically approach the 
true fundamental eigenvalue of the medium (Figure 4.9). As long as $G$ is sufficiently large, TORTE obtains a good approximation of the fundamental eigenvalue: for the prompt supercritical medium, it is within $1 \%$ for the worst case $G=40$, and varies well within $0.5 \%$ for the rest of the runs. While the calculated fundamental eigenvalue has a distinct trend with increasing $G$, the calculated delayed eigenvalues show little effect from this increase. This is expected because nothing about the delayed neutron precursors changes in the different runs.

The behavior of a complex eigenvalue as $G$ increases is more difficult to measure because it moves in two-dimensional complex space (Figure 4.10). For the first two runs of the prompt supercritical medium, TORTE calculates a real eigenvalue because of the small $G$. As the $G$ continues to increase, this quickly becomes a complex eigenvalue and begins to converge to a final point in the spectrum, as evidenced by a decrease in the change of the eigenvalue between runs for large $G$.

The eigenvalue spectra of the delayed supercritical and subcritical media exhibit similar behavior as $G$ increases. For all runs of the subcritical medium, the calculated prompt fundamental eigenvalues vary well within $0.5 \%$, with the best agreement over the last four with the largest $G$, runs E through $\mathrm{H}$ (Table 4.6). For all runs of the delayed supercritical medium, the fundamental and prompt fundamental eigenvalues

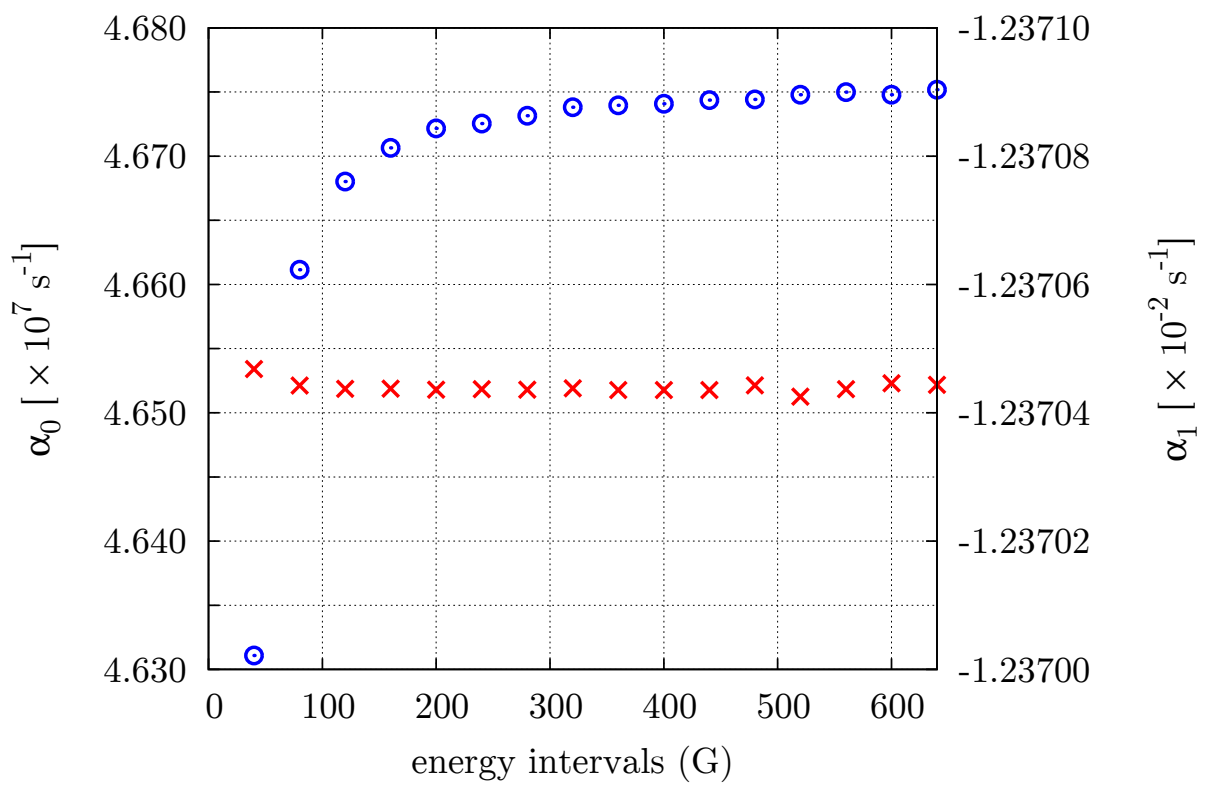

prompt fundamental $\odot \quad$ first delayed $\times$

Figure 4.9: Two eigenvalues calculated with increasing numbers of energy intervals for Problem 6 shows the prompt eigenvalues asymptotically approaching a value, while the delayed eigenvalues remain the same. 


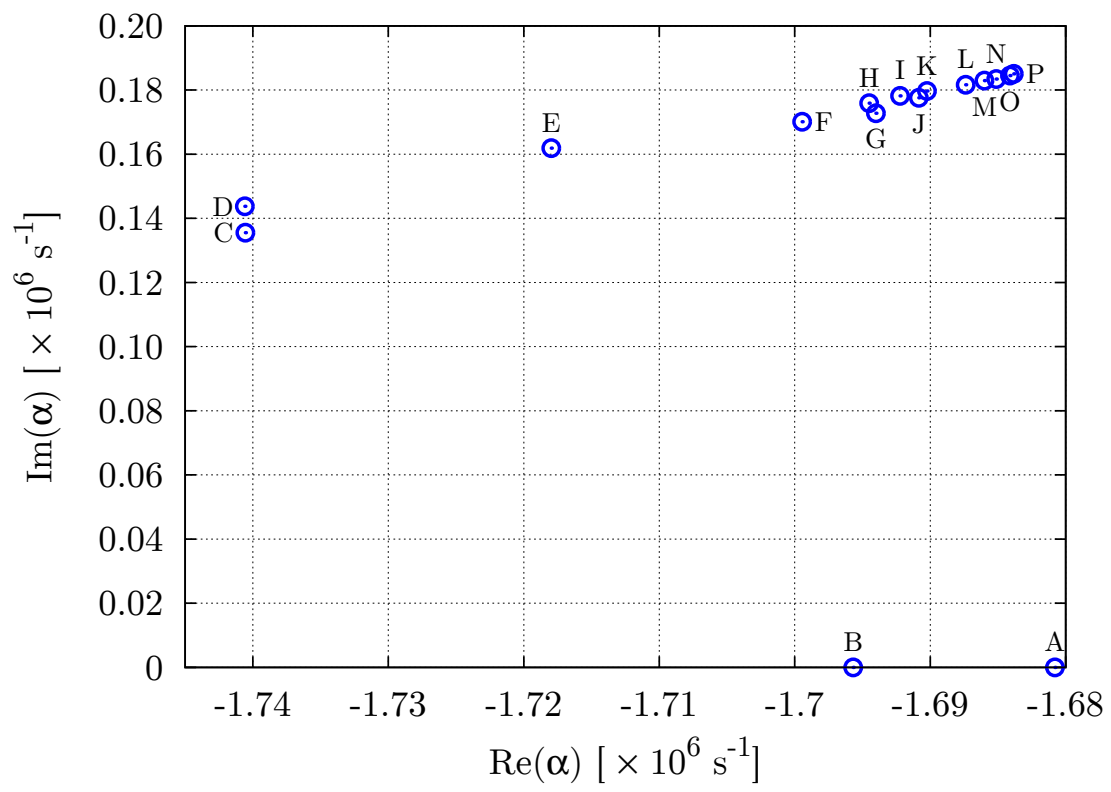

Figure 4.10: The first calculated complex eigenvalue of Problem 6 approaches a value as the number of energy intervals increases. With 640 energy intervals, the final calculation of this prompt eigenvalue yields $\alpha_{p 1}=\alpha_{7}=(16.838+1.8508 i) \times 10^{5} \mathrm{~s}^{-1}$.

vary less than $1 \%$, with the prompt fundamental eigenvalue showing better agreement (Table 4.7). This is due to the very small magnitude of the fundamental eigenvalue: it is three orders of magnitude smaller than the prompt fundamental eigenvalue. The next four prompt eigenvalues show less agreement but look to be converging to a single value.

Measuring the trend of these higher eigenvalues as $G$ increases is difficult due to the existence of both discrete points and lines in the true $\alpha$-eigenvalue spectrum: it

Table 4.6: Problem 8 energy intervals and calculated prompt fundamental $\alpha$ eigenvalues. Units are $\mathrm{s}^{-1}$.

\begin{tabular}{ccc}
\hline Run & $G$ & $\alpha_{p 0}=\alpha_{6}$ \\
\hline A & 80 & -942.11 \\
B & 320 & -940.48 \\
C & 640 & -940.10 \\
D & 1000 & -939.71 \\
E & 1500 & -940.60 \\
F & 2000 & -940.72 \\
G & 2500 & -940.53 \\
H & 3000 & -940.67 \\
\hline
\end{tabular}


Table 4.7: Problem 7 energy intervals, calculated fundamental $\alpha$ eigenvalue, and first five prompt $\alpha$ eigenvalues. Units are $\mathrm{s}^{-1}$.

\begin{tabular}{cccccccc}
\hline Run $^{\mathrm{a}}$ & $G$ & $\alpha_{0}$ & $\alpha_{p 0}=\alpha_{6}$ & $\alpha_{7}$ & $\alpha_{8}$ & $\alpha_{9}$ & $\alpha_{10}$ \\
\hline $\mathrm{A}$ & 40 & 0.068954 & -78.734 & -177030 & -208060 & -215110 & -219780 \\
$\mathrm{~B}$ & 80 & 0.069413 & -78.606 & -175030 & -207160 & -214540 & -217570 \\
$\mathrm{C}$ & 160 & 0.069540 & -78.572 & -174490 & -206890 & -214370 & -217870 \\
$\mathrm{D}$ & 320 & 0.069568 & -78.554 & -174360 & -206810 & -214330 & -216890 \\
\hline
\end{tabular}

a TORTE uses the same random number seed in calculations of these eigenvalues.

is first necessary to determine whether a higher calculated eigenvalue is discrete or part of a line. It is known that some eigenvalues are discrete: the delayed eigenvalues and in most cases the prompt fundamental eigenvalue. Other discrete eigenvalues are identified empirically, e.g., regardless of $G$, the complex eigenvalues on the edges of the spectrum of the prompt supercritical medium (Figure 4.8) converge to the same point, and as TORTE calculates more eigenvalues of the spectrum, none fall between these existing complex eigenvalues. Other calculated higher eigenvalues shift and trace out lines as TORTE calculates more eigenvalues in the spectrum, especially in cases for very large $G$. This is the case for the subcritical medium, where the next highest prompt eigenvalues shift with increasing $G$. With the existence of lines in the spectrum, it is presumed that $G$ never exceeds the number of eigenvalues in the true spectrum.

While the fundamental eigenvalue of the delayed supercritical medium is also the fundamental delayed eigenvalue, it follows a trend similar to a prompt fundamental eigenvalue as $G$ increases (Figure 4.9). The other five calculated delayed eigenvalues for this problem show no trend related to $G$ (Table 4.8), despite the fact that they are different than the decay constants. The trend in these eigenvalues is like that seen for the delayed eigenvalue (Figure 4.9).

Table 4.8: Problem 7 calculated delayed $\alpha$ eigenvalues. Units are $\mathrm{s}^{-1}$.

\begin{tabular}{cccccc}
\hline Run & $\alpha_{d 1}=\alpha_{1}$ & $\alpha_{2}$ & $\alpha_{3}$ & $\alpha_{4}$ & $\alpha_{5}$ \\
\hline A & -0.013468 & -0.049047 & -0.15763 & -1.1387 & -8.0454 \\
B & -0.013466 & -0.049006 & -0.15756 & -1.1385 & -8.0445 \\
C & -0.013465 & -0.048995 & -0.15754 & -1.1384 & -8.0442 \\
D & -0.013465 & -0.048993 & -0.15753 & -1.1384 & -8.0442 \\
\hline
\end{tabular}




\subsubsection{Eigenfunctions}

Along with the $\alpha$ eigenvalues, TORTE calculates $G$ prompt and $J$ delayed eigenfunctions. The most important of these for the expanded flux solution correspond to the first few longest-lived eigenvalues: a few prompt and the delayed eigenfunctions. An adjoint eigenfunction represents the importance of a neutron in establishing the corresponding kinetic mode. For the prompt supercritical medium, the energy spectrum of the asymptotic solution is hard and it peaks near $2 \mathrm{MeV}$ (Figure 4.11). Because few neutrons reach very low energies, noise is present in eigenfunctions where $E<0.1 \mathrm{eV}$. Compared to the prompt eigenfunction, the energy spectrum of the delayed eigenfunction is softer, with an increase in the thermal energy range due to the emission spectrum of delayed neutrons: the difference between these eigenfunctions is kinetic distortion. All delayed eigenfunctions have a similar shape. Because fast fission dominates the medium, the neutrons highest in energy are the most important to the asymptotic solution. The importance varies at some energies due to resonance absorption: a neutron at an energy within a resonance is likely to be absorbed, so it has little importance. Due to the large variation in the prompt fundamental eigenfunction, it is difficult to see the effect that the ${ }^{235} \mathrm{U}$ absorption resonances have on

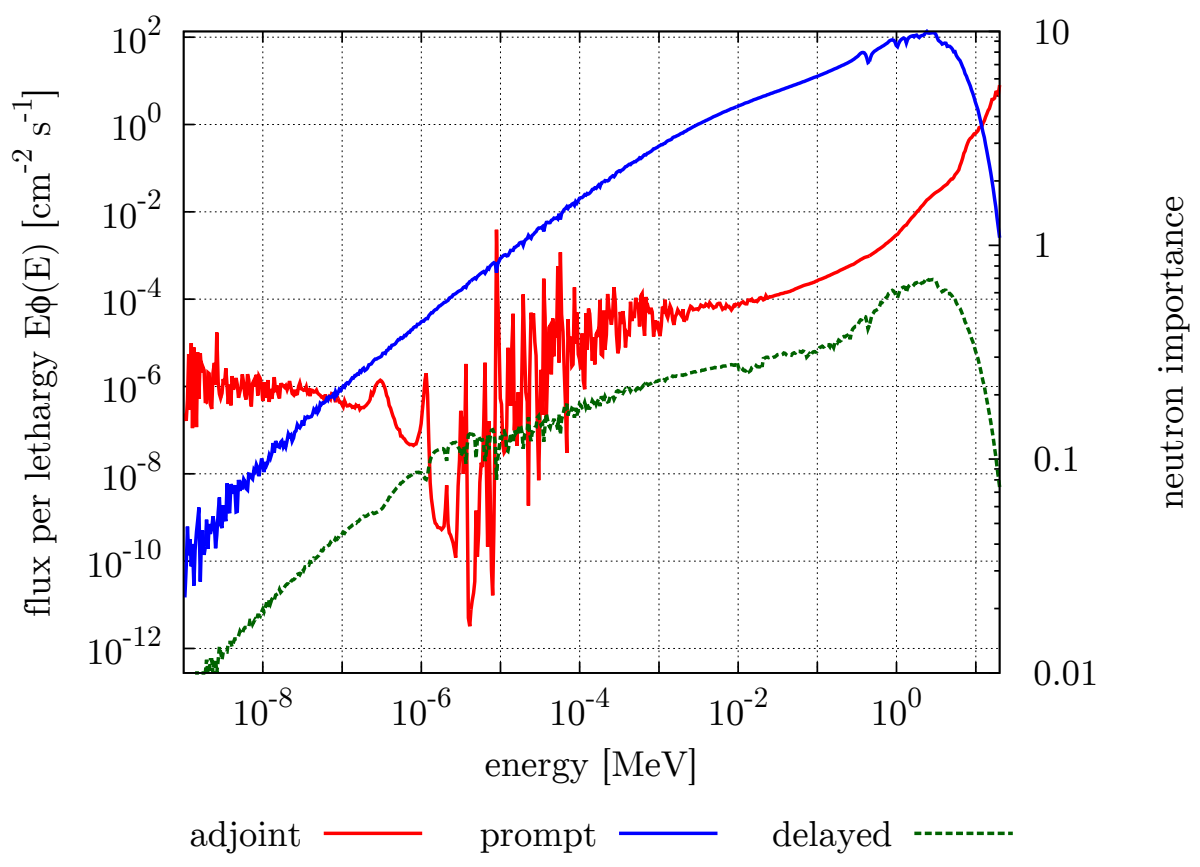

Figure 4.11: Selected eigenfunctions of the 640 energy interval Problem 6 show the hard energy spectrum of the asymptotic solution, kinetic distortion of the delayed eigenfunction, and the importance of high-energy neutrons in a fast, prompt supercritical, infinite medium. 
the forward eigenfunctions (Figure 4.12).

The prompt fundamental forward and adjoint eigenfunctions of the subcritical, graphite-moderated medium (Figure 4.13) show the effect of neutron thermalization on the asymptotic solutions. In a thermal medium, the energy spectrum is much softer and the most important neutrons are those that induce the thermal fissions that drive the medium. High-energy neutrons have low importance due to the likelihood of absorption in the resonance region during thermalization in this subcritical medium. The eigenfunctions also have flux depressions from the ${ }^{238} \mathrm{U}$ absorption resonances, and there is little noise at lower energies due to neutrons populating these energies during the Monte Carlo random walk. All delayed eigenfunctions are nearly identical to that of the prompt fundamental mode: the effect of kinetic distortion is negligible in this far subcritical medium. The eigenfunctions of the delayed supercritical medium have some similar characteristics.

Eigenfunctions of higher $\alpha$ eigenvalues have positive and negative parts, and some are complex. The next two highest eigenfunctions of the subcritical medium (Figure 4.14) correspond to the eigenvalues $\alpha_{7}=-8504.7 \mathrm{~s}^{-1}$ and $\alpha_{8}=-8807.6 \mathrm{~s}^{-1}$, and cross the energy-axis multiple times. The highest eigenfunctions vary wildly and are sharply peaked in only a few energy intervals. Complex eigenfunctions show similar behavior to the other higher eigenfunctions when plotting the real and imaginary

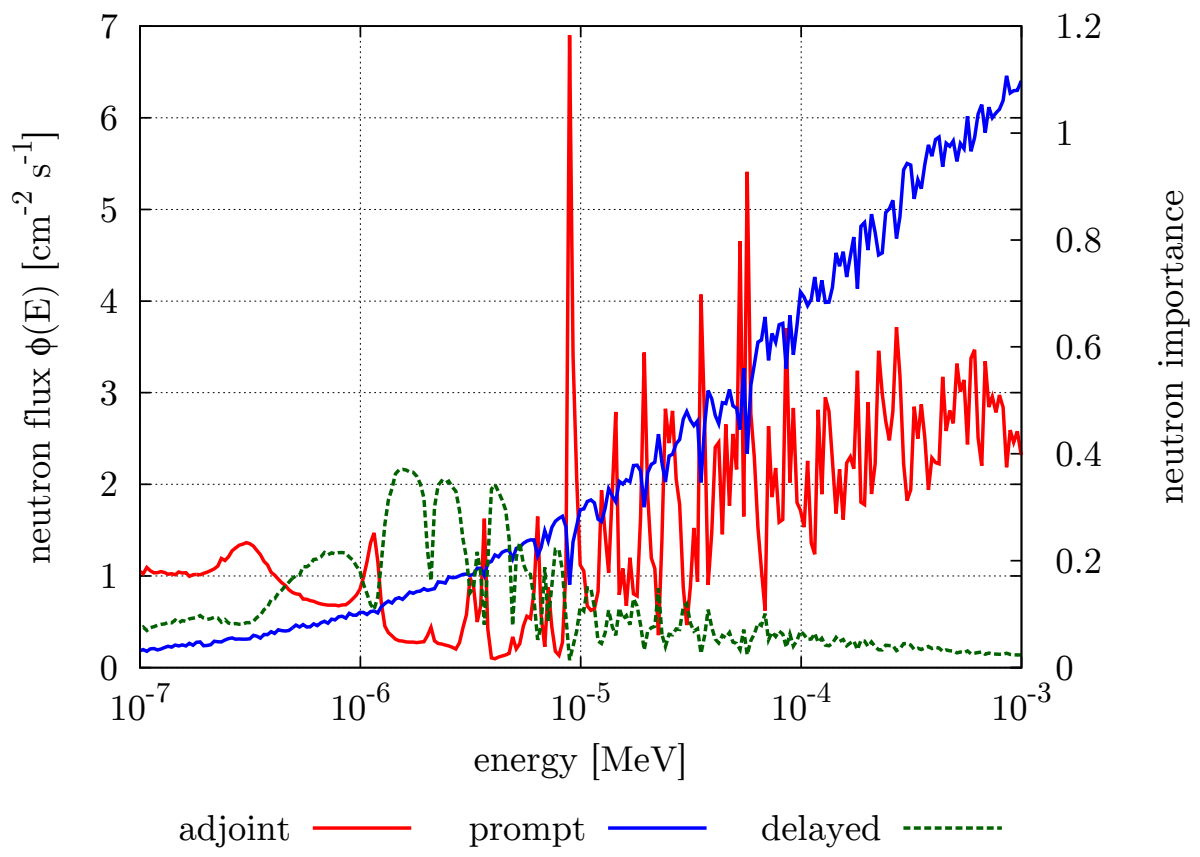

Figure 4.12: An alternate visualization of the eigenfunctions of the 640 energy interval Problem 6 show the effect of the resonances on the forward and adjoint eigenfunctions. 


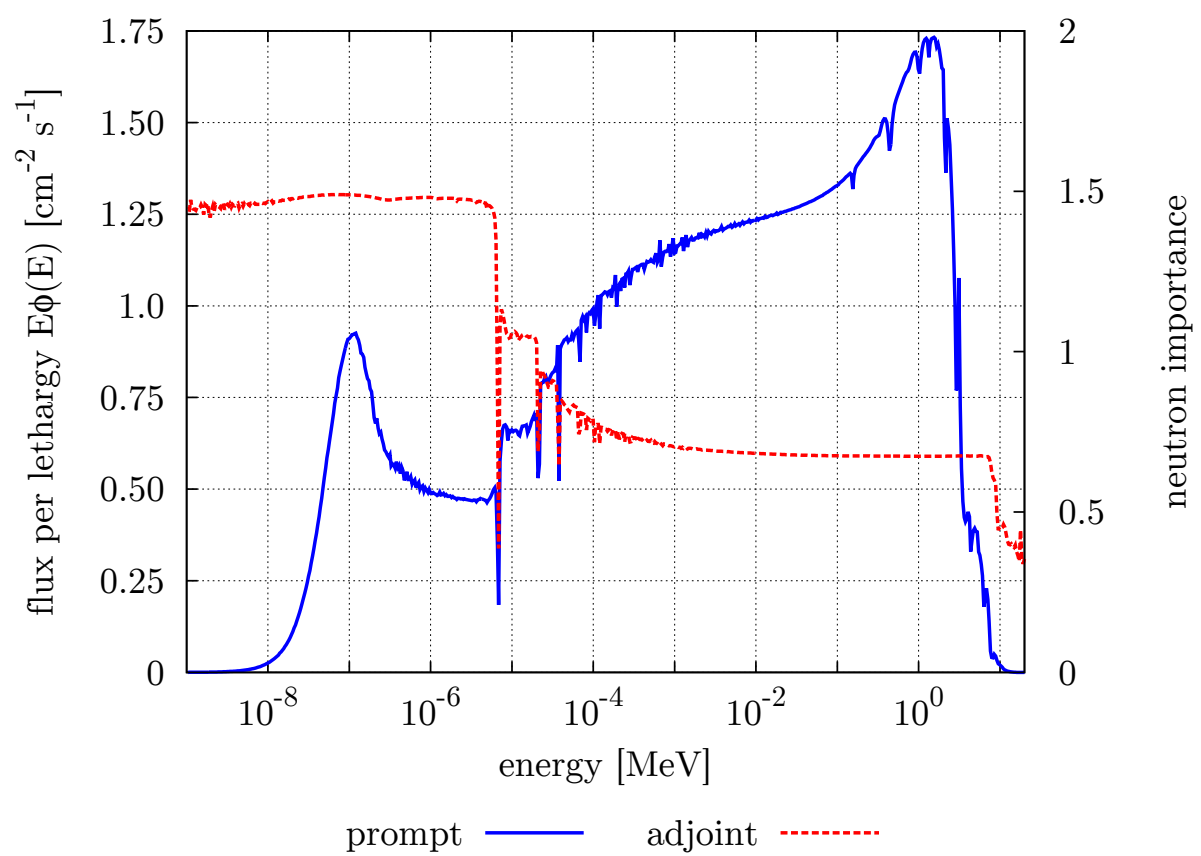

Figure 4.13: The fundamental forward and adjoint eigenfunctions of the 3000 energy interval Problem 8 show the soft spectrum of the asymptotic solution and the importance of thermal neutrons in a thermal, graphite-moderated, subcritical infinite medium.

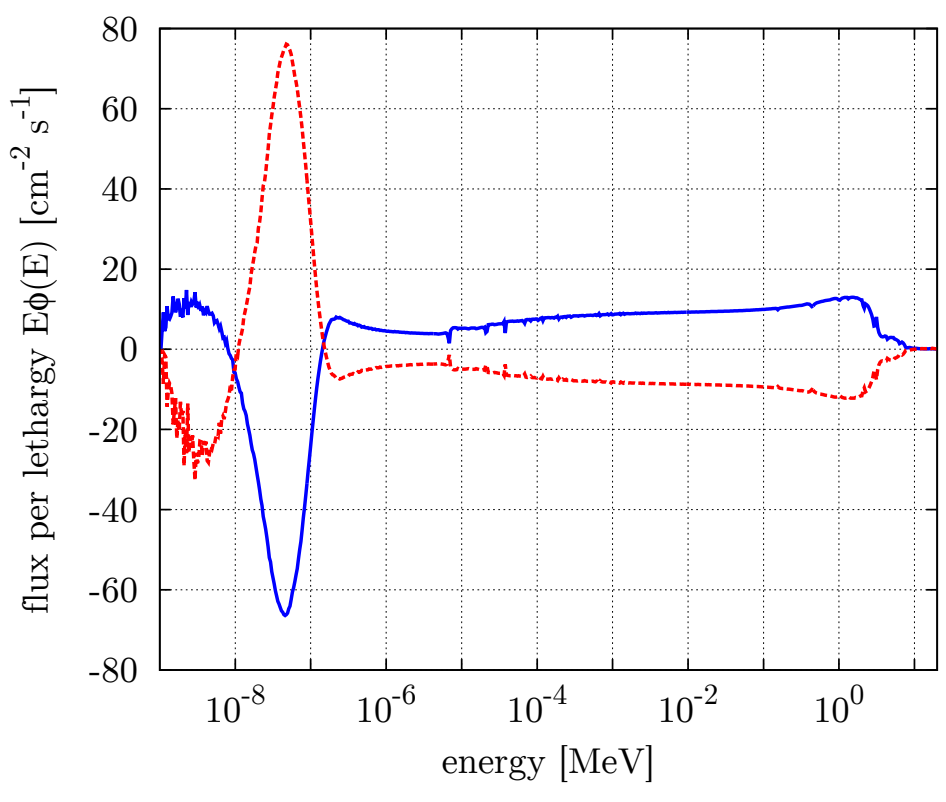

first prompt $\longrightarrow$ second prompt

Figure 4.14: The next highest all-real, prompt forward eigenfunctions of the 3000 energy interval Problem 8 have similar shapes with positive and negative parts. 
parts individually in the energy space.

\subsubsection{Eigenvalue Convergence}

Using the prompt supercritical medium, it was shown that the TRMM calculates the same eigenvalue spectrum for a given material composition and $G$, and that these calculated eigenvalues converge to the true spectrum, in order, as $G$ becomes large. It is now left to discuss the effect of Monte Carlo tally statistical errors on calculated results and the effect that the energy phase space discretization has on these results. For the $G=320$ energy interval run of the delayed supercritical medium, TORTE builds the TRM and calculates the eigenvalues after each of 200 active cycles, which run $5 \times 10^{5}$ particles per cycle. This medium is unique because the $k$ eigenvalue is so close to unity: it is more important than in the prompt supercritical or subcritical media to have the $k$ eigenvalue converged within a few pcm. This has a large effect on the calculated $\alpha$ eigenvalue, because for a near-critical medium, it is very close to zero. Thus, the delayed supercritical medium presents a more challenging problem to the TRMM than the other two media.

The elements $q_{i j}$ of the TRM are tallied quantities that have some associated statistical error. With more histories, the statistical uncertainty of the tallied removal rates and probabilities, and thus the $q_{i j}$ 's, decreases. With more energy intervals, fewer events occur within each interval for the same number of histories, so the resulting $q_{i j}$ 's have a greater statistical uncertainty.

For the delayed supercritical medium, the fundamental and prompt $\alpha$ eigenvalues converge in approximately $25 \times 10^{6}$ active histories, after which statistical variations are relatively minimal (Figure 4.15). The L2-norms of the eigenfunctions

$$
|\vec{\psi}|_{2}=\sqrt{\psi_{1}^{2}+\psi_{2}^{2}+\cdots+\psi_{G}^{2}}
$$

follow a similar trend. Calculated delayed eigenvalues change little over the active histories as they mostly depend on the calculation of the decay constant: this is the sampling of the decay time of the precursors. The fundamental and prompt

eigenvalues depend on prompt neutron effects. The calculated fundamental eigenvalue varies close to $7 \%$ over the last half of the active histories, but because it is so close to zero, this is a poor measure of convergence. In absolute terms, it varies less than $5 \times 10^{-3} \mathrm{~s}^{-1}$ over the last 100 cycles. The next higher prompt eigenvalues only vary close to $0.1 \%$ over the same range, but the absolute variation is five orders of magnitude greater than that of the fundamental eigenvalue. The prompt fundamental 


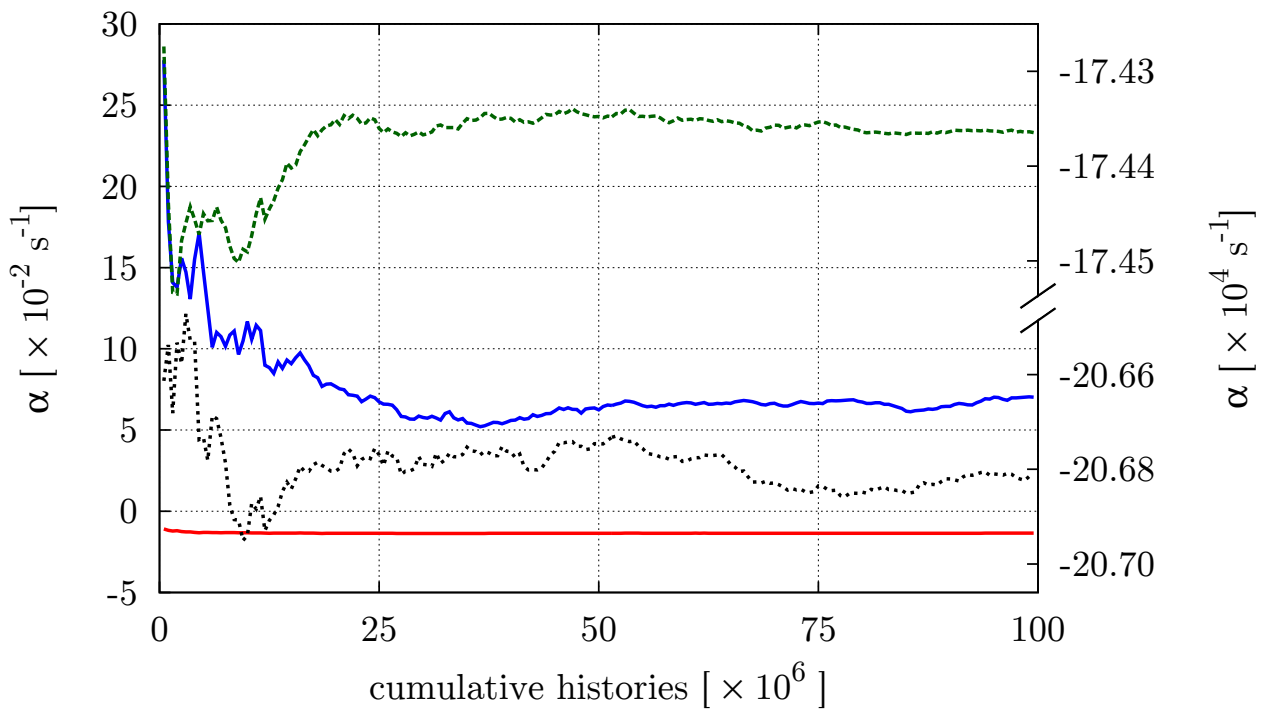

Fundamental $\longrightarrow$ First Prompt
Largest Delayed $\quad \begin{array}{r}--\cdot-\cdots \cdot . \\ \text { Second Prompt }\end{array}$

Figure 4.15: Four of the first ten eigenvalues calculated after each active cycle converge as the number of active histories increases for the 320 energy interval Problem 7 . The prompt eigenvalues shown are seven orders of magnitude larger than the fundamental eigenvalue.

eigenvalue also has a similar trend. Other than the prompt fundamental and delayed eigenvalues, all higher $\alpha$ eigenvalues are greater than $10^{5} \mathrm{~s}^{-1}$.

Examination of the static reactivity of the medium helps in understanding the large relative variation in the converging fundamental $\alpha$ eigenvalue. Using Eq. (2.83), the reactivity is $0.00177 \pm 6.8 \%$. This is a measure of the deviation from critical, and for a delayed supercritical medium, there is a large uncertainty because the $k$ eigenvalue is only on the order of a hundred pcm above critical. In the prompt supercritical medium, this same uncertainty in the $k$ eigenvalue only leads to a $0.01 \%$ uncertainty in the calculated reactivity. These similarities between the statistical uncertainty of the reactivity and the variation of the calculated fundamental $\alpha$ eigenvalue are not surprising: examine the relationship in the inhour equation in Eq. (2.104). Thus, the fundamental eigenvalue in a prompt supercritical or far subcritical medium has a smaller relative variation over the last half of the active histories than the eigenvalue of this near-critical medium.

These assertions are supported with additional calculations using problem 7, where ten runs begin with different random number seeds (Figure 4.16). In all cases, the calculation of the eigenvalues varies greatly for the first few cycles, followed by less erratic behavior as the calculated values of each run draw closer together as the 


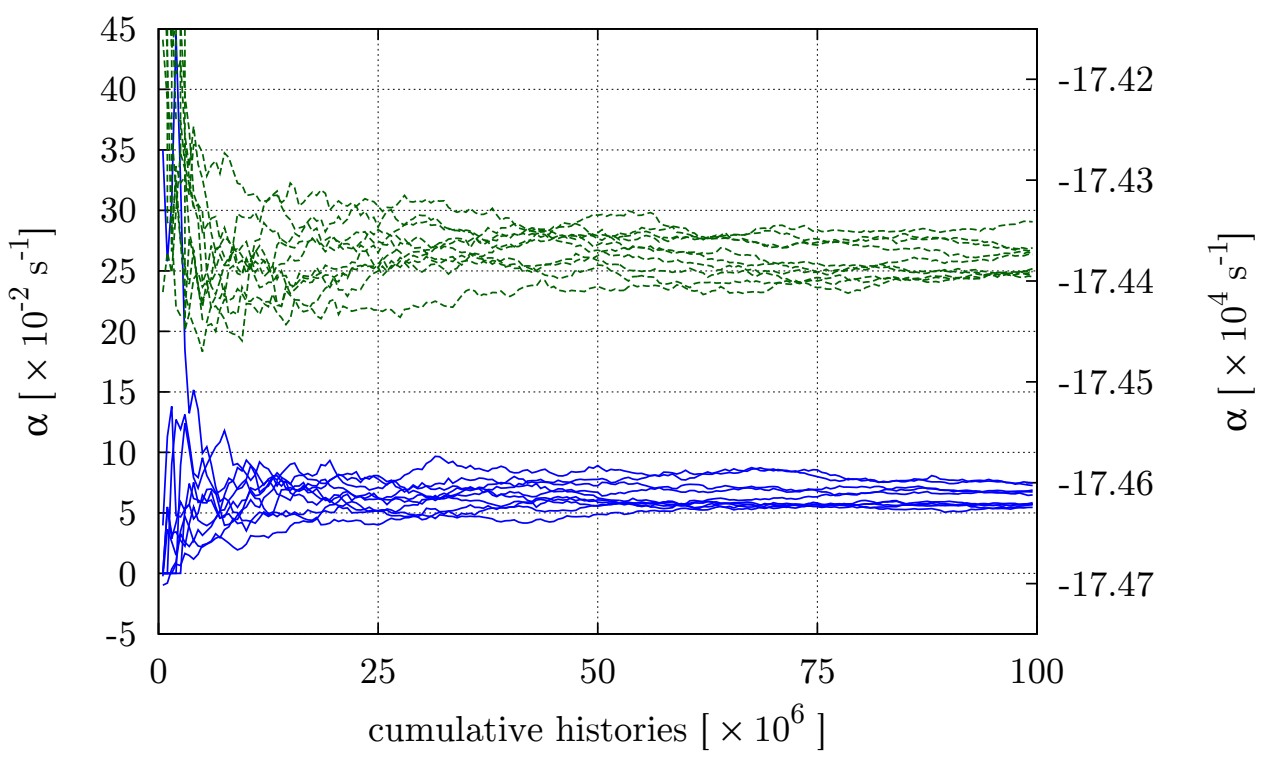

Fundamental — First Prompt

Figure 4.16: The convergence behavior of two eigenvalues calculated with ten different random number seeds shows the range of individual realizations caused by the random walk. These are two of the first ten eigenvalues of the 320 energy interval Problem 7 . The prompt eigenvalue is seven orders of magnitude larger than the fundamental eigenvalue.

number of active histories increases. As mentioned, the calculated fundamental $\alpha$ eigenvalues from these runs have a larger relative range, as they are correlated to the calculation of the reactivity. For the first prompt eigenvalue, the largest and smallest calculated values vary less than $0.05 \%$. This type of analysis is useful in establishing the degree of confidence in calculated results.

To demonstrate the effect of $G$ on the convergence rate, TORTE calculates spectra of the delayed supercritical medium with the same energy phase space discretizations (Table 4.7) using the same random number seed: physically, the same sequence of events occur in all runs. The only differences are the tallies and the size of the TRM. As $G$ decreases, the statistical uncertainty on the $q_{i j}$ 's decrease as well, and though the calculated eigenvalue spectra are different for all runs, the first few eigenvalues converge to similar quantities. For these similar eigenvalues, the convergence rate is nearly identical: the convergence trends of the calculated fundamental eigenvalues are so similar for all $G$, that on a plot, they line up on one another. This is counterintuitive because with less statistical uncertainties in the elements of the TRM, it is reasonable to expect a faster convergence. But, characterization of the TRM is more about the global behavior of the problem as a whole than any individual part. 
One important measure is how these statistical variations or different spectra affect the expanded flux solutions. The effect of a statistical variation on a given $\alpha$ eigenvalue is different for two cases: $\alpha>0$ and $\alpha<0$. A positive $\alpha$ eigenvalue is often the fundamental eigenvalue of a supercritical medium. Consider a medium with the true positive fundamental eigenvalue $\alpha_{0}$ and a calculated eigenvalue $\alpha_{0}-\epsilon$, where $\epsilon>0$. Assuming equal coefficients $A$, the absolute difference $f^{+}(t)$ between using these two eigenvalues in the eigenfunction expansion is

$$
f^{+}(t)=A \exp \left(\alpha_{0} t\right)[1-\exp (-\epsilon t)]
$$

As time increases, $f^{+}(t)$ grows exponentially. For example, the fundamental eigenvalues of the delayed supercritical medium calculated after 100 active cycles and all 200 active cycles are $0.00644 \mathrm{~s}^{-1}$ and $0.00695 \mathrm{~s}^{-1}$, respectively. At $t=10 \mathrm{~s}, f^{+}(t)$ for these eigenvalues is $0.5 \%$. The fundamental eigenvalues from the prompt supercritical medium calculated with $G=600$ and $G=640$ energy intervals are $4.6747 \times 10^{7}$ $\mathrm{s}^{-1}$ and $4.6751 \times 10^{7} \mathrm{~s}^{-1}$, respectively. At $t=103 \mathrm{~ns}, f^{+}(t)$ for these eigenvalues $0.5 \%$. This is because, even though the prompt supercritical medium fundamental eigenvalues have a better relative agreement than those for the delayed supercritical medium, the absolute difference is more important to the eigenfunction expansion. Still, the majority of the short-time behavior in the flux solution occur during which $f^{+}(t)$ is small.

Most eigenvalues in the spectra have negative real parts. Consider a medium with the true negative eigenvalue $\alpha$ and a calculated eigenvalue $\alpha+\epsilon$, where $\epsilon>0$. Assuming equal coefficients $A$, the absolute difference $f^{-}(t)$ between using these two eigenvalues in the eigenfunction expansion is

$$
f^{-}(t)=A \exp (\alpha t)[\exp (\epsilon t)-1]
$$

In contrast to when $\alpha$ is positive, in the limit as $t \rightarrow \infty, f^{-}(t)$ approaches zero. There is a finite time $t>0$ where $f^{-}(t)$ is maximized:

$$
t=\frac{1}{\epsilon} \ln \left(\frac{\alpha}{\alpha+\epsilon}\right) .
$$

Thus, $f^{-}(t)$ for any error in a calculated negative $\alpha$ eigenvalue is bounded.

The differences in the $\alpha$-eigenvalue spectra due to $G$ and statistical errors has a lesser effect on the expanded flux solution than the stark difference in individual eigenvalues suggests. For this delayed supercritical medium, TORTE expanded flux 
solutions using different $G$ agree on the short-time evolution of the energy spectrum from the slowing down of neutrons from a pulse source (Figure 4.17). At $t=0.015 \mu \mathrm{s}$, neutrons in all runs downscatter at equal rates and align along the same line. Flux depressions form in the high-energy range as neutrons downscatter past resonances.
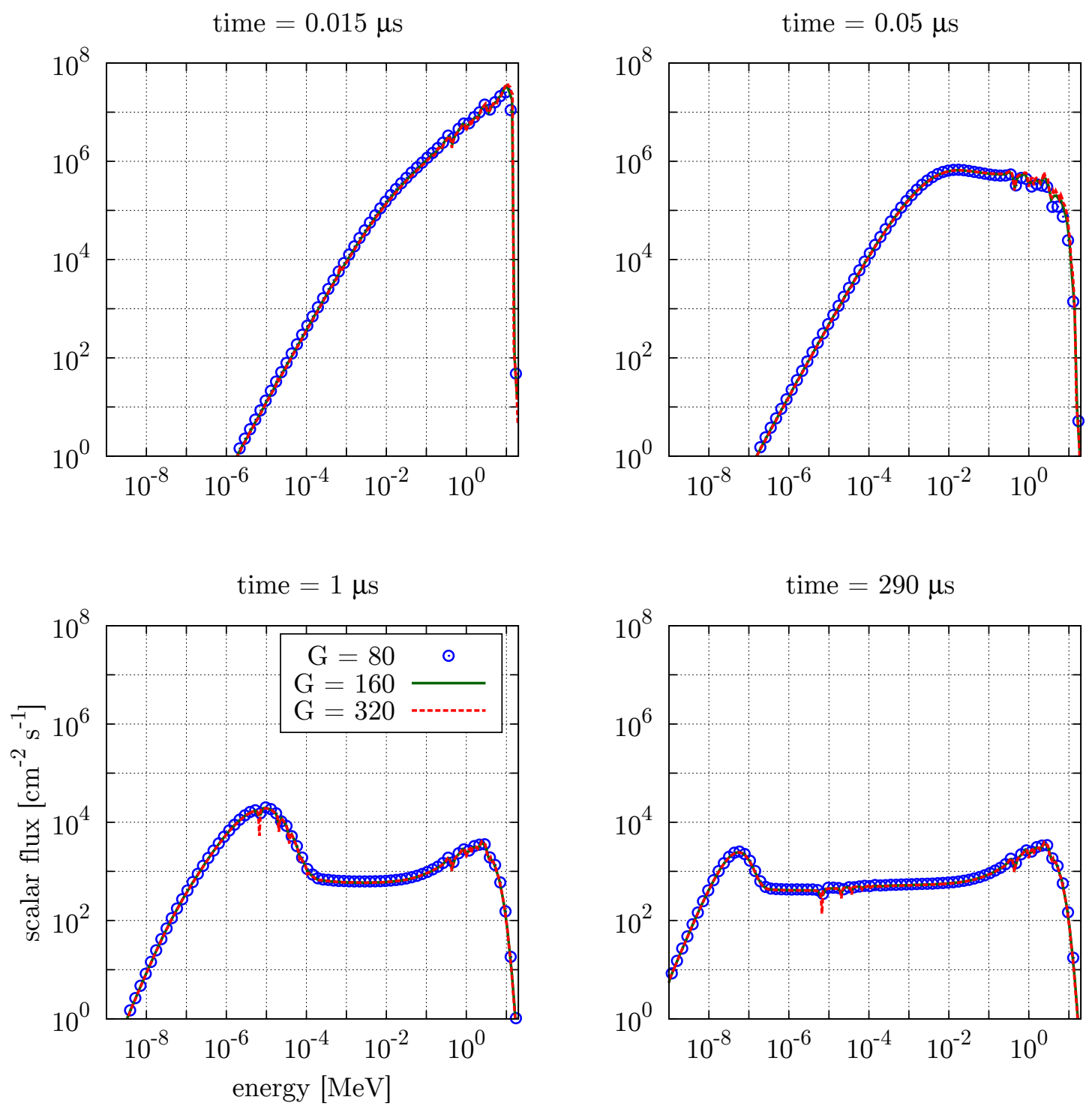

Figure 4.17: Snapshots of the Problem 7 expanded flux solutions using all calculated modes show the agreement of the short-time evolution of the flux using different numbers of energy intervals during the TRM calculation. The initial source is a pulse of strength $Q_{0}=1 \times 10^{15}$ $\mathrm{cm}^{-3} \cdot \mathrm{s}^{-1}$ at $t_{0}=0$ without initial precursor concentrations. TORTE approximates a $E_{0}=$ 14.1 MeV source, defining the source in the $g$ th energy interval for which $E_{g+1} \leq E_{0}<E_{g}$. Due to the interval widths in the energy phase space discretizations, this initial source is slightly different for all three runs, but the effect this has on the expanded solution is insignificant. 
Because neutrons are able to downscatter to near-zero energies in a collision with hydrogen, the flux extends to lower energies at short times. At $t=0.05 \mu \mathrm{s}$, neutrons continue to downscatter as the high-energy flux begins to decrease. A very broad flux packet forms. The effect of resonances is more resolved with more energy intervals. The run B flux still has depressions due to resonances, but they are less pronounced. At $t=1.0 \mu \mathrm{s}$, despite the fact that this is a supercritical medium, the high-energy flux continues to decrease because neutrons downscatter out of the high energy range faster than they induce fissions that emit neutrons at these energies. Lower energy resonances cause dips in the flux packet as neutrons downscatter. At $t=290.0 \mu \mathrm{s}$, all higher prompt kinetic modes have decayed, and the prompt fundamental mode and eigenvalue dictates the energy spectrum and time behavior of the solution. Only the fundamental and delayed kinetic modes have non-negligible contributions to the expanded flux solution at this time. The prompt fundamental kinetic mode continues to dominate the flux solution until it begins to decay at approximately $1 \mathrm{~ms}$. At this time, the delayed kinetic modes and eventually the positive fundamental kinetic mode dominate the flux solution. In any case, the flux energy spectrum remains the same, as the shape of the delayed and prompt fundamental eigenfunctions is the same.

For most of the time, the expanded solutions using different $G$ are nearly indiscernible. A difference in the solutions is only noticeable after several minutes to an hour, and is caused by the different calculated fundamental eigenvalues of the runs. Realistically, if this medium is left unchanged for an hour, temperature and material density changes render this solution invalid.

\subsubsection{Comparison to Time-Dependent Monte Carlo}

To measure the accuracy of the eigenfunction expansion, a modified TORTE code generates the true TDMC solution for a given source. This code is named after its purpose, To Obtain Real Time Behavior (TORTB).

\section{Code (TORTB)}

TORTB is simply a modified version of TORTE designed to run a fixed-source calculation. Because both codes run on the same set of subroutines, the code physics and energy phase space discretization is the same. This allows for an fair verification of the ability of the eigenfunction expansion to correctly approximate the time-dependence of the flux. TORTB uses censusing to obtain the flux spectrum at specific times. Each census represents a time boundary, at which the neutron history is stopped 
and released in the next cycle. These censuses are chosen such that they match the expanded flux solution.

The initial inputs to TORTB are source definitions: the energy and time distribution, and the number of histories emitted from the source. TORTB builds a source bank sampling from the appropriate distributions. For a monoenergetic pulse source, it starts all source neutrons at the same energy and at $t_{0}=0$. TORTB then follows the censusing technique outlined in $\S 2.2 .4$. With a more complex time-dependent source, TORTB begins tracking a neutron when $t_{n-1}<t_{0}<t_{n}$, where $t_{n}$ are ordered census times. It uses no variance reduction techniques, and does not re-sample from a source distribution at each census. A run ends when neutrons hit the specified last census or if all neutron histories terminate. For a supercritical medium, the last census must be specified. During the run, if a fission occurs, a prompt fission neutron energy is immediately sampled and the neutron track continues instantaneously: delayed fission is not modeled. After each census, TORTB prints the current distribution of neutron energies. The result is a file with the energy distribution of the neutron flux at each time census.

\section{Flux Transient Comparison}

TORTE expanded flux solutions are able to preserve the detail in the energy spectrum and match well to TDMC calculations throughout transients (Figure 4.18). At $t=$ $0.03 \mu \mathrm{s}$, neutrons from the monoenergetic pulse begin to downscatter and form a distinct flux packet, as neutrons colliding with carbon do not scatter to near-zero energies. Some noisy behavior is observed in the eigenfunction expansion for this initial short time period $t<0.05 \mu \mathrm{s}$. The TDMC calculation handles this short time period well and has a smooth solution. At $t=0.15 \mu \mathrm{s}$, the flux packet takes on a smoother form as neutrons collide with the graphite moderator. The flux at higher energies decreases rapidly, as neutrons are not at energies at which fission replenishes the high-energy flux. The TDMC solution has noisy behavior at high energies due to low-probability, high-energy neutrons born from fast fissions. This distinct flux packet maintains its form and continues to propagate to lower energies. At $t=4 \mu \mathrm{s}$, the flux packet reduces in size as the fast neutron flux continues to decrease. The flux packet encounters low energy resonances: flux depressions caused by ${ }^{238} \mathrm{U}$ capture resonances are resolved in both solutions due to large $G$. The depth of the depressions are less pronounced in the TDMC solution. At $t=100 \mu \mathrm{s}$, the flux packet continues to decrease as the spectrum begins to assume the shape of the prompt fundamental kinetic mode. Noise present in the high energy range of 
the TDMC solution is due to the decreased neutron population leading to the fewer fissions that are necessary to replenish the high-energy flux. The energy spectrum approaches the prompt fundamental kinetic mode on the order of a few hundred $\mu$ s. After this time, the flux shape remains the same: only the magnitude changes. The prompt fundamental mode decays after a few ms, after which delayed fission neutrons
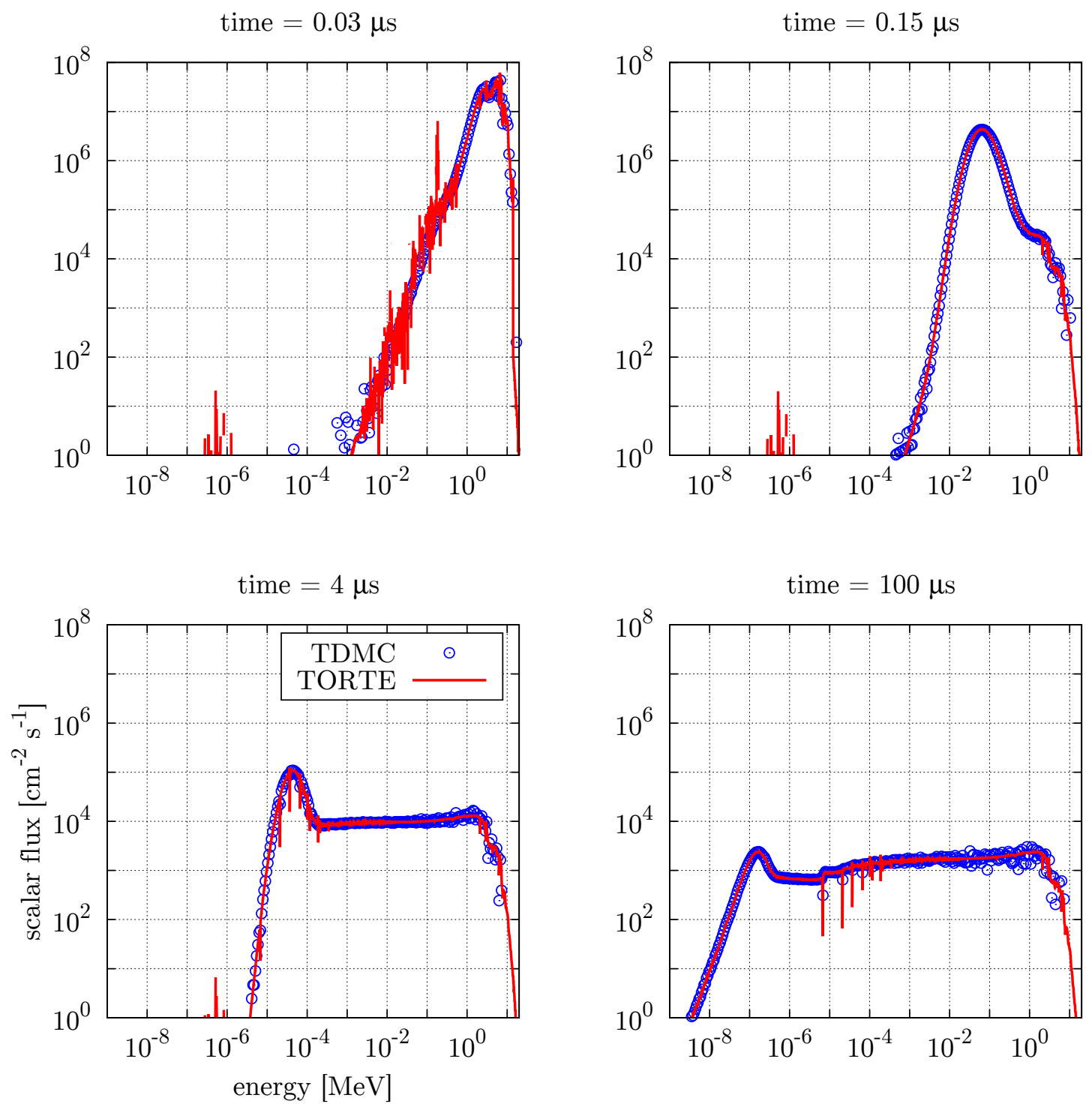

Figure 4.18: Snapshots comparing the expanded flux solution to the TDMC solution for Problem 8 shows agreement throughout the transient for a graphite-moderated, subcritical problem reacting to a $14.1 \mathrm{MeV}$ pulse without initial precursor concentrations. TORTE uses 1000 energy intervals, the source strength is $Q_{0}=1 \times 10^{15} \mathrm{~cm}^{-3} \cdot \mathrm{s}^{-1}$, and the expansion uses all calculated kinetic modes. The TDMC calculation uses 320 energy intervals and starts with $40 \times 10^{6}$ neutron histories at exactly $E=14.1 \mathrm{MeV}$. The effect that the difference in the initial sources has on the time-dependent solutions is negligible. 
and their decay constants dictate the time-dependent change in the flux.

The eigenfunction expansions of the delayed supercritical and subcritical media (Figures 4.17 and 4.18) show the differences caused by the moderating nuclei. The flux packet is far more defined in the graphite-moderated subcritical medium (Figure 4.18) because the neutrons have a minimum, non-zero energy to which they downscatter. This also contributes to the flux at higher energies decreasing at an earlier time. The flux at higher energies in the graphite-moderated medium tends to oscillate more: it decreases as neutrons slow down from high energies, rises as thermal neutrons fission and emit high-energy neutrons, and decreases again to approach the fundamental mode solution. The hydrogenous medium has an increase in the flux in the high-energy range. In both media, the prompt fundamental mode solution remains stationary as higher modes decay. This far more physical solution is in contrast to the 81-group expanded flux solution. Even for the prompt supercritical medium, most of the higher modes decay before the the fundamental mode increases. Most of the interesting energy-dependent flux effects occur within a few milliseconds for the thermal media.

The accuracy of the expanded flux solutions show the potential for their use to approximate the time-dependent behavior of the flux. Higher kinetic modes decay to the fundamental mode solution only after a few milliseconds: during this initial time, the fundamental mode solution is a very inaccurate representation of the flux solution.

\subsubsection{Quantification of the Residual}

Little is known about the residual term in the eigenfunction expansion described by Eq. (2.103) other than it decays rapidly. The assertions gained in this section are from empirical observations of the eigenfunction expansion using the TRMM. While these provide some insight to the behavior of the $\alpha$-eigenvalue spectrum, it is not a rigorous mathematical derivation. All three continuous-energy problems exhibit some residual behavior for very short time periods.

Without an analytic solution for the time-dependent flux, and because the TDMC solutions are subject to statistical noise, it is difficult to determine the full residual $\zeta(E, t)$. But, theoretically, the residual is at its maximum at $t=0$ and because the initial source is known, $\zeta(E, 0)$ is easily obtained by defining Eq. (2.103) at $t=0$,

$$
\psi(E, 0)=\sum_{i=0}^{G+J-1} \frac{\psi_{i}^{\dagger}(E) \cdot Q_{0}(E)}{\gamma_{i}} \psi_{i}(E)+\zeta(E, 0) .
$$


The initial flux $\psi(E, 0)$ calculated by the eigenfunction expansion is affected by this residual regardless of the initial source (Figure 4.19): the residual term is not an artifact of attempting to model a sharp flux shape with the eigenfunction expansion. With a monoenergetic source, the solution peaks in the appropriate energy interval, but has an additional spectrum of noisy behavior, where there are negative parts of the expanded flux solution. Even with a smoother initial source, there is noise in the higher energy range, where $0.1 \mathrm{MeV}<E<2 \mathrm{MeV}$. Subtracting the expected initial flux as calculated from the source definition yields the initial residuals (Figure $4.20)$, which oscillate with negative and positive parts. The overall shapes of these two residuals only differ slightly at very low energies below $E<0.01 \mathrm{eV}$ for the same medium.

From the smoothness of the expanded flux solutions for early times, it is evident that this large residual term decays quickly. It also decays at different times for the three media: for the subcritical medium, there is noise in the expanded flux solution for relatively long times (Figure 4.18) compared to the smooth solution for the delayed supercritical medium at an earlier time (Figure 4.17). In the prompt supercritical medium, the expansion shows no effect of the residual term relatively immediately at $t \ll 10 \mathrm{~ns}$. The residual in the delayed supercritical medium is observed to decay at

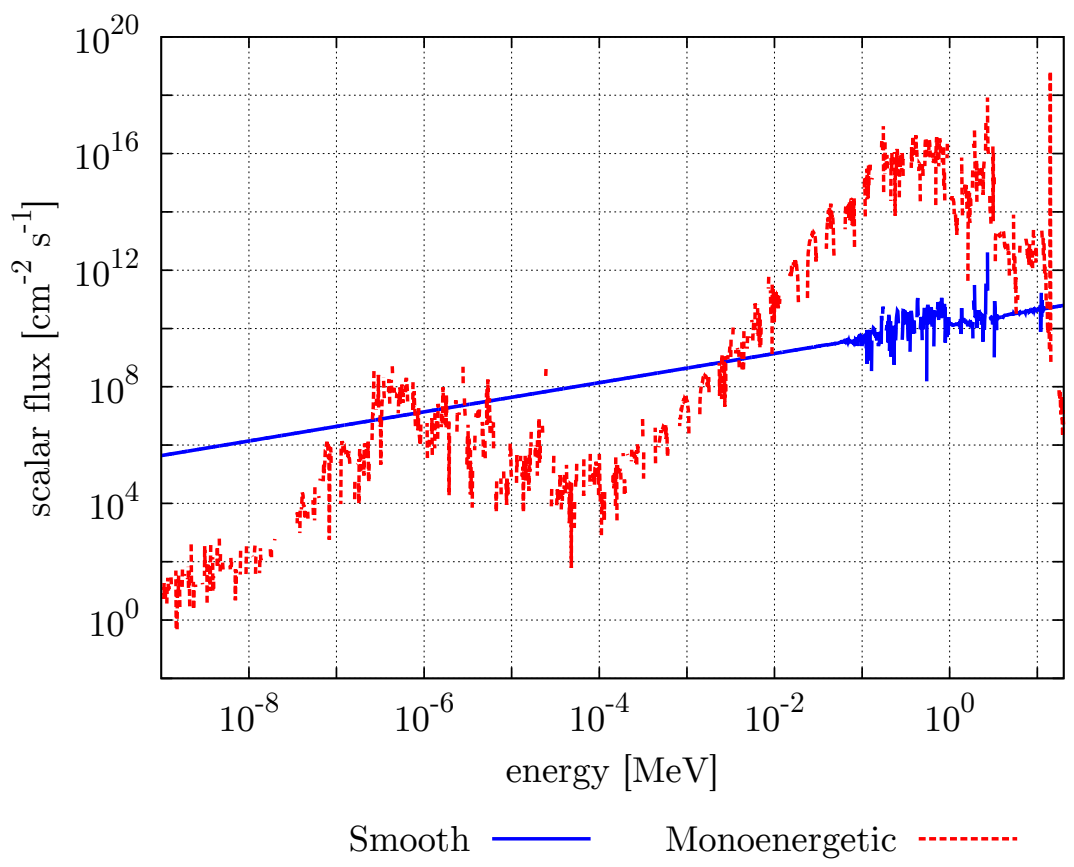

Figure 4.19: Initial $t=0$ fluxes from the 3000 energy interval Problem 8 eigenfunction expansion show the magnitude of the missing residual term. The monoenergetic source is a $14.1 \mathrm{MeV}$ pulse and the smooth source is distributed equally among all energy intervals. 


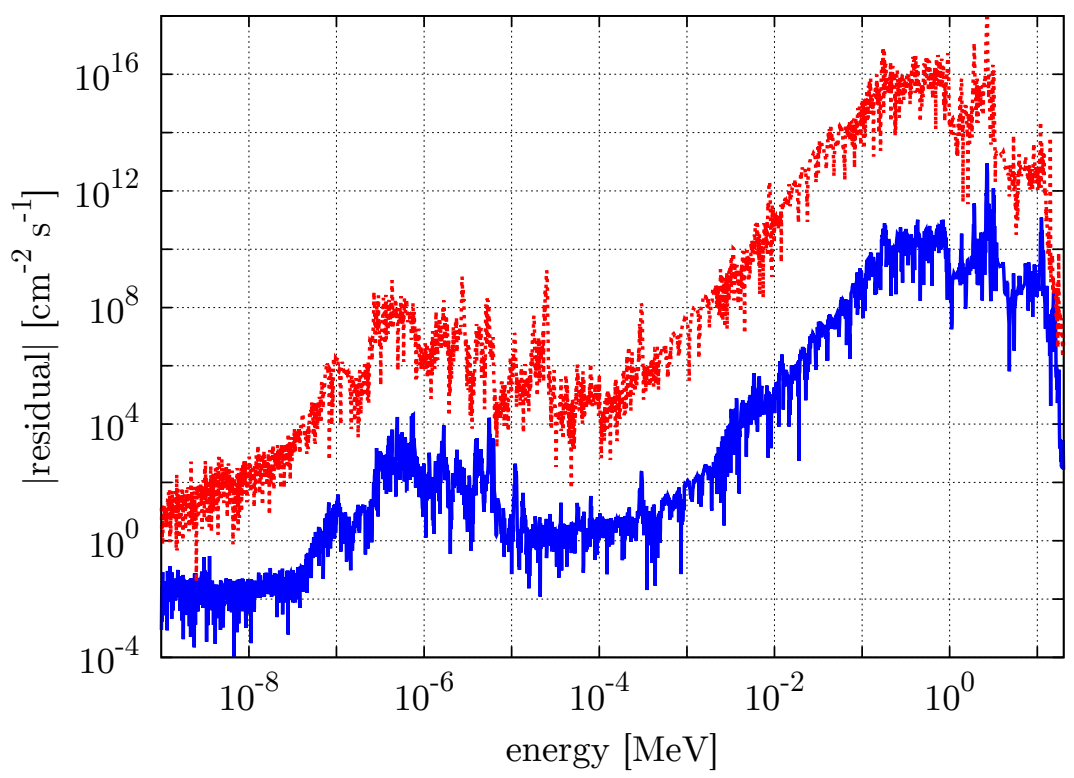

Smooth

Monoenergetic

Figure 4.20: The absolute value of the residual terms of the 3000 energy interval Problem 8 expanded flux solutions using two different initial sources have nearly identical shapes and the same magnitude relative to the initial source.

a time an order of magnitude earlier than for the subcritical medium, as the residual in the prompt supercritical medium is observed to decay at a time several orders of magnitude earlier. Along with these different observed decay times, the residuals for each of the media have different shapes (Figure 4.21).

The relative difference in these observed decay rates are on a similar relative scale to the neutron generation times $\Lambda$ for similar types of media [8]: for a normal LWR, $\Lambda \approx 1 \times 10^{-5} \mathrm{~s}^{-1}$; for a normal graphite-moderated or heavy-water reactor, $\Lambda \approx 10^{-4}$ $\mathrm{s}^{-1}$; and for a fast reactor, such as a mixed-oxide fueled ( $\left.\mathrm{Pu}-\mathrm{U}\right)$ reactor, $\Lambda \approx 4.9 \times 10^{-7}$ $\mathrm{s}^{-1}$. Rearranging the inhour equation in Eq. (2.104) for the neutron generation time yields

$$
\Lambda=\frac{\rho-\beta_{\mathrm{eff}}}{\alpha_{p 0}},
$$

where the static reactivity is defined in Eq. (2.83). Because TORTE calculates the $k$-eigenvalue and there is no leakage in an infinite medium, this equation yields the calculated neutron generation times of these media (Table 4.9). These times match theoretical expectations and the relative scales of each match the difference in the observed decay times.

These observations and some additional calculations lead to some conclusions on 


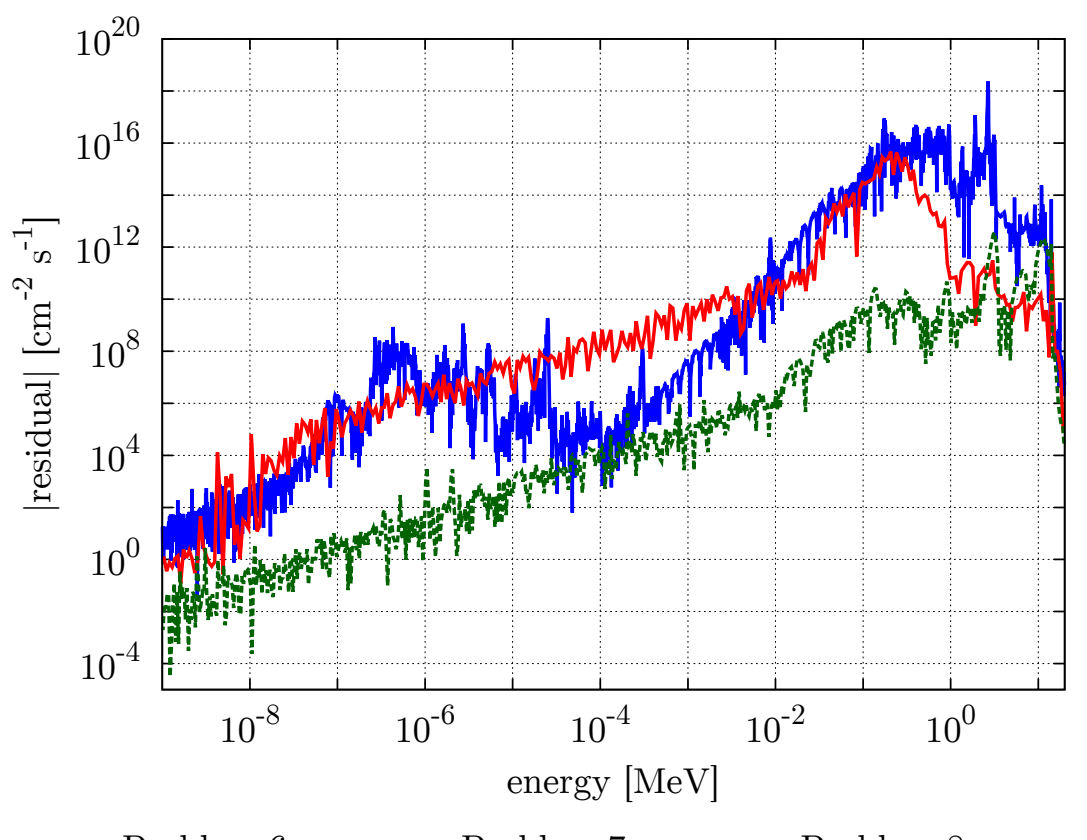

Problem 6 -------.. $\quad$ Problem 7 Problem 8

Figure 4.21: The absolute value of the initial residual terms for the three continuous-energy media have different shapes due to the material definitions.

this observed residual term. In all media, the residual decays well before one neutron generation time, but the relative observed decay time between the three problems is similar to the differences in the neutron generation times. The residual is not a result of the shape of the source, but depends on the material composition of the problem. But, if the fundamental eigenfunction $\left[v^{-1} \psi_{i}(E) C_{i}\right]$ is set to the initial source, the residual term disappears: the forward and adjoint eigenfunctions are bi-orthogonal to machine precision. For any $G$ from the subcritical medium runs, all calculate a residual with the same shape, and even with $G=3000$ kinetic modes, the residual is still present. If this residual is due to an insufficient number of modes calculated, it

Table 4.9: A comparison of calculated neutron generation times of the three continuousenergy media.

\begin{tabular}{clcccc}
\hline Problem & Description & $\Lambda[\mu \mathrm{s}]$ & $\rho$ & $\alpha_{p 0}\left[\mathrm{~s}^{-1}\right]$ & $\beta_{\text {eff }}[\%]$ \\
\hline 6 & Fast, Very & 0.01097 & 0.51936 & $4.675 \times 10^{7}$ & 0.64 \\
& Supercritical & & & & \\
7 & Thermal, Delayed & 60.27 & 0.001766 & -78.55 & 0.65 \\
& Supercritical & & & & \\
8 & Thermal, Very & 166.5 & -0.15011 & -940.7 & 0.65 \\
& Subcritical & & & & \\
\hline
\end{tabular}


would at least change as $G$ gets large. Also, the effect of the use of forward weighting for the adjoint-weighted elements diminishes as $G$ gets large. Statistical uncertainties in the calculated TRM do not contribute to the residual. There is no residual in the 81-group medium expanded flux solution (Figure 4.3), even though Monte Carlo methods obtain the rates in the TRM. Also, for the $G=3000$ run of the subcritical medium, TORTE recalculates the eigenfunction expansion with and increased number of neutron histories with no change in the initial shape of the residual.

\subsection{Discussion}

The research Monte Carlo code TORTE implements the TRMM for infinite media to demonstrate its ability to accurately calculate $\alpha$ eigenvalues and use the entire spectrum to obtain expanded flux solutions. For five multigroup problems, TORTE is able to calculate the $k$ eigenvalue, TRM, and $\alpha$ eigenvalue spectrum. These calculated results match analytic solutions, and demonstrate the applications of eigenfunction expansion. For three continuous-energy problems, TORTE converges to an $\alpha$ eigenvalue spectrum with an increase in the number of active histories. It calculates discrete delayed and prompt fundamental eigenvalues in line with theoretical expectations. TORTE converges to a fundamental $\alpha$ eigenvalue with an increase in the number of energy intervals $G$. Some eigenvalues begin to trace out lines in the complex plane for very large $G$. Regardless of $G$, expanded flux solutions agree on the time-dependent shape of the flux until long after the fundamental modes dominate the spectrum. These flux solutions match TDMC calculations. A discussion of some characteristics of the initial residual term $\zeta(E, 0)$ shows that it appears at very early times in the expanded flux solutions, suggesting incompleteness of the calculated eigenfunctions.

\section{References}

[1] MATLAB, version 7.10.0. Natick, Massachusetts: The Mathworks Inc., 2010.

[2] FORTRAN90. American National Standards Institute, 1991.

[3] E. Anderson, Z. Bai, C. Bischof, S. Balackford, J. Demmel, J. Dongarra, J. Du Croz, A. Greenbaum, S. Hammarling, A. McKenney, and D. Sorensen, LAPACK Users' Guide. Philadelphia, Pennsylvania: Society for Industrial and Applied Mathematics, Third ed., 1999.

[4] T. Williams and C. Kelley, "gnuplot 4.6: An Interactive Plotting Program," 2012.

[5] X-5 Monte Carlo Team, "MCNP - A General N-Particle Transport Code, Version 
5, Volume I: Overview and Theory," Tech. Rep. LA-UR-03-1987, Los Alamos National Laboratory, 2003.

[6] E. W. Larsen, "NERS 543: Advanced Nuclear Reactor Theory." University of Michigan, Lecture Notes, Fall, 2008.

[7] E. W. Larsen, Personal Correspondence, 2012.

[8] K. O. Ott and D. A. Menely, Introductory Nuclear Reactor Dynamics. La Grange Park, Illinois: American Nuclear Society, 1985. 


\section{CHAPTER 5}

\section{Eigenvalues and Eigenfunctions of Slabs}

This chapter discusses the implementation of the TRMM to one-dimensional media, i.e., slabs, where the TRM characterizes neutrons moving through a simplified position-direction-energy phase space. With the single position variable $x$ and angular variable $\mu$ defined as the $x$-direction cosine, slab geometry is the simplest way of implementing spatial variables to the TRMM and benchmarking results. Methods discussed in this chapter extend directly to two- or three- dimensional media, with a requirement of more memory. Problems consist of slabs of thicknesses $\Delta$ with vacuum boundary conditions at the left and right boundaries. A reflecting, white, or periodic boundary condition eliminates higher shape eigenfunctions and eigenvalues from the solution. This is not desirable, except in very specific cases where the configuration, desired initial source, and detectors, i.e., the desired information to be received, all reflect the effect of the special boundary condition. In any other case, all higher shape eigenfunctions are necessary to obtain accurate time-dependent solutions.

A research Monte Carlo code calculates the TRM and determines eigenpairs of the forward and adjoint matrices, handling both multigroup and continuous-energy problems. The Green's Function Method (GFM) provides five one-speed problems for verification of TRMM-calculated eigenvalues and eigenfunctions. It forms a matrix problem from equations describing the interactions of the boundary angular fluxes, as outlined in $\S$ 2.5.3. Parametric studies using these problems show the effect of the discretization of the position and direction phase spaces on the calculated $\alpha$ eigenvalues in the absence of the treatment of the energy phase space. TDMC solutions for the flux in these one-speed slabs verify the TRMM eigenfunction expansion and show the effect the continuum spectrum has on the residual term $\zeta(x, t)$ from Eq. (2.103). Applications to continuous-energy slabs demonstrate the ability of the TRMM to obtain

$\alpha$ eigenvalues and deliver time-dependent solutions for more realistic problems. This includes discussions on the dependence of continuous-energy $\alpha$ eigenvalue spectra on 
selections of the phase space discretizations.

\subsection{Codes (TORTE-1D and TORTB-1D)}

The infinite-medium FORTRAN90 [1] code version of TORTE is modified to include the spatial variables and routines necessary for one-dimensional Monte Carlo transport, and is appropriately renamed TORTE-1D. For multiplying media, the code still performs a $k$-eigenvalue power iteration to tally removal rates and events for the TRM. In purely-scattering media, the $k$-eigenvalue is zero: the code uses the $c$-eigenvalue power iteration to obtain a source for the active Monte Carlo cycles. TORTE-1D calculates the collision estimates of the $k$ and $c$ eigenvalues, but with the additional leakage in slab geometry, these calculated eigenvalues have a weaker correlation to the calculated fundamental $\alpha$ eigenvalue. For mixed media, e.g., media with fissile fuel regions and scattering reflectors, TORTE-1D calculates the $k$-eigenvalue.

For continuous-energy problems, the energy after collision determines the scattering direction of neutrons for free gas and elastic scattering [2]. For continuous-S $(\alpha, \beta)$, TORTE-1D samples a direction isotropically instead of sampling from the tables. It assumes isotropic prompt and delayed fission emission and still uses LAPACK [3] routines for finding eigenpairs of the forward and adjoint matrices. The code only treats one-dimensional heterogeneous or homogeneous finite slabs of thickness $\Delta$ with vacuum boundary conditions at $x=0$ and $x=\Delta$. For the one-speed verification, TORTE-1D further simplifies to include only position and direction variables. All scattering and fission emission of these problems is isotropic. The physics of the simplified code TORTE is described in $\S 4.1$.

Like TORTE-1D, TORTB-1D is derived from the infinite medium FORTRAN90 code TORTB to provide TDMC solutions for verification. TORTB-1D runs a fixedsource calculation, obtaining the time-dependent flux distribution with a census technique. At each census, TORTB-1D prints the scalar neutron flux for each position and energy interval: it essentially integrates the angular flux over the direction phase space. To reduce memory demands in handling very supercritical slabs, TORTB-1D runs separate batches with a smaller number of neutron histories, storing the flux at censuses and aggregating them at the end of all batches. Other information on the code TORTB is described in $\S$ 4.3.5. The results refer to TORTE-1D and TORTB-1D by their shortened names. 


\subsubsection{Tallying the Transition Rate Matrix}

TORTE-1D tallies removal rates and probabilities for the TRM entries in much the same way for continuous-energy media, but considers the $N$ slabs and $M$ direction intervals of the problems. It defines states as a neutron moving in direction interval $m$, energy interval $g$, and position interval (slab) $n$ or a precursor in position interval (slab) $n$. All tallies are based on removal rates

$$
\tau_{i}^{-1}=(\text { average removal time from state } i)
$$

and most entries are combinations of these rates, probabilities, and multipliers, such as

$$
\begin{aligned}
v_{i} \Sigma_{f i} & =\tau_{i}^{-1}\left(\frac{\# \text { of removals due to fission of neutrons in state } i}{\# \text { of removals from state } i}\right), \\
v_{i} \Sigma_{s i j} & =\tau_{i}^{-1}\left(\frac{\# \text { of removals due to scatters from state } i \text { into state } j}{\# \text { of removals from state } i}\right) .
\end{aligned}
$$

TORTE-1D calculates all entries of the TRM from tallied quantities during the Monte Carlo random walk except for the delayed neutron emission direction. Because delayed neutron emissions are already rare events, for any particular precursor group, TORTE-1D only tallies $\chi_{d}(E)$ during the random walk and distributes the angular emission probabilities isotropically using the knowledge of number of direction intervals. While it is possible to treat other quantities of the TRM similarly, the rest of the TRM uses tallied distributions, e.g., for prompt fission, it tallies the angular dependence $\chi_{p}(\mu, E)$ instead of assuming that prompt fission is isotropic. This provides for checks of the tallied $\chi_{p}(\mu, E)$ that it is isotropic within some statistical uncertainty.

For the slab problems, the discretized position and direction phase spaces consist of equal-sized intervals. TORTE-1D defines the slab boundaries such that they match the material boundaries of each problem, instead of defining slabs that contain different materials. These selections are unnecessary limitations, but they simplify much of the analysis. The number of direction intervals is always even to avoid an angular interval in the direction parallel to the slab faces. TORTE-1D maintains the equal-lethargy interval discretization for the energy phase space.

As the TRM grows in size, an issue with empty states arises, i.e., when the size of the phase space intervals decrease, sometimes a neutron never exists in a given state during the Monte Carlo random walk. This is not an issue in continuousenergy infinite media and one-speed slabs, but with continuous-energy slabs and larger 
systems, several states are empty, especially with mixed media where the existence of high-energy neutrons from fission is improbable at the regions near the edge of the reactor. The simple solution is to eliminate the state from the TRM entirely: because a neutron does not enter the state during a Monte Carlo random walk, it is unlikely a neutron enters the state during a transient. The only time this treatment becomes an issue is if a desired source for the eigenfunction expansion is in one of these empty states. In this case, the adjoint eigenfunctions do not operate on the desired source. Another solution is merging an empty state with an adjacent state, which requires careful bookkeeping.

\subsubsection{Eigenvalues and Eigenfunctions}

TORTE-1D calculates up to $N(M G+J) \alpha$ eigenvalues and shape eigenfunctions for the forward and adjoint matrix equations depending on the number of fissile slabs $N_{f}$. For purely-scattering slabs, which lack precursor concentrations, TORTE-1D calculates only $N M G \alpha$ eigenvalues and shape eigenfunctions. The shape eigenfunctions are vectors of length $N(M G+J)$, where the organization of the states determines the order of the entries of the shape eigenfunctions. These entries are the angular flux dependent on $M$. Summing the entries over the angular intervals yields the scalar flux for a given energy $g$ and slab $n$,

$$
\phi_{n g}=\sum_{m=1}^{M} \psi_{n g m}
$$

This sum yields a vector of length $N G+N_{f} J$ containing the position- and energydependent shape eigenfunctions organized by slab $n$,

$$
\left[\begin{array}{ll}
\phi(E) & C
\end{array}\right]_{n}=\left[\begin{array}{llllllll}
\phi_{1} & \phi_{2} & \cdots & \phi_{G} & C_{1} & \cdots & C_{J-1} & C_{J}
\end{array}\right]_{n}
$$

for $n=1,2, \ldots, N$. If slab $n$ is purely scattering, the last $J$ entries are removed and the vector in Eq. (5.5) is of length $G$. The eigenfunction expansion coefficients $A_{i}$ are dot products of the adjoint shape eigenfunctions and desired initial source

$$
A_{i}=\frac{\psi_{i}^{\dagger}(x, \mu, E) \cdot Q_{0}(x, \mu, E)}{\gamma_{i}}
$$

The ability of the expansion to model sharp monoenergetic or directional sources is tied to the chosen phase space discretizations used to calculate the adjoint shape eigenfunctions. 


\subsubsection{Visualization of Flux Solutions}

With additional phase space variables, solutions depended on the entire positionenergy-direction phase space. Obtaining the scalar flux eliminates the direction phase space dependence, and for one-speed media, there is only one energy interval. The TRM still includes the direction information, but it is unnecessary in many applications. For continuous-energy media, results shown are linear combinations of the flux to deliver a quantity similar to an ideal detector response,

$$
R_{d}=\left\langle\Sigma_{d}(x, E), \phi(x, E)\right\rangle,
$$

where the selection of the simulated cross sections $\Sigma_{d g}$ reflect the detector material, such as a fissile isotope or boron (Table 5.1). In most cases, this response depends mostly on the thermal neutron flux.

Table 5.1: Simulated cross sections for obtaining the ideal detector response.

\begin{tabular}{ccccc}
\hline$g$ & 1 & 2 & 3 & 4 \\
\hline$\Sigma_{d g}$ & 0.01 & 0.02 & 0.06 & 0.91 \\
\hline
\end{tabular}

\subsection{One-Speed Verification}

For five one-speed slabs, calculated $\alpha$-eigenvalue spectra and eigenfunction expansion solutions from the TRMM are benchmarked to the GFM results and TDMC solutions. The GFM [4] described in $\S 2.5 .3$ calculates the real $\alpha$-eigenvalues and shape eigenfunctions of scattering or multiplying one-dimensional media. Unless otherwise noted, TORTE sets the neutron speed to $v=1 \mathrm{~cm} / \mathrm{s}$ and total cross sections to unity $\Sigma_{t}=1 \mathrm{~cm}^{-1}$ to match the GFM specifications. Thus, the resulting calculations are dimensionless and the problem thicknesses are in mean free paths (mfp). With a single energy interval, the TRMM results depend purely on the choice of position and direction intervals.

The TORTE-calculated $\alpha$-eigenvalue spectra have many eigenvalues omitted from the benchmark: most of these are complex and exceed the Corngold limit in the negative direction, $\alpha<\alpha^{*}$, which identifies the start of the continuum as discussed in $\S 2.3 .3$. The GFM searches for the discrete real eigenvalues in the spectrum individually and does not search beyond this limit, which is $\alpha^{*}=-1$ for most problems in the benchmark. For TORTE-calculated results, the real spectrum consists of the real eigenvalues that exist before the start of the calculated complex part of the spectrum. 
A discussion on the calculation of complex points within the continuum follows the benchmark results.

\subsubsection{Non-Multiplying Media}

Problems 9A-E: These are one-speed, purely-scattering, homogeneous slabs of thicknesses ranging from $\Delta=1.0$ to $\Delta=25.0 \mathrm{mfp}$. The TORTE-calculated, real $\alpha$-eigenvalue spectrum agrees with the GFM-calculated eigenvalues (Table 5.2). For slabs of all thicknesses, the fundamental eigenvalues show the best agreement. This agreement tightens with increasing slab thickness, despite the shrinking magnitude of the eigenvalue and the same number of total eigenvalues calculated in all spectra. Also, the difficulty of calculating the highest eigenvalues increases with slab thickness. In the $\Delta=20$ and $\Delta=25 \mathrm{mfp}$ slabs, TORTE has difficulty computing the eigenvalues closest to the Corngold limit before the start of the complex portion of the eigenvalue spectrum. In problems where the highest eigenvalue is much smaller than the others, TORTE has more difficulty computing it: in the $\Delta=5$ and $\Delta=10 \mathrm{mfp}$ slabs, the eigenvalues closest to the Corngold limit show the most difference to the GFM eigenvalues. In the $\Delta=20$ and $\Delta=25 \mathrm{mfp}$ slabs, TORTE does not even calculate these eigenvalues before calculating complex eigenvalues. These complex eigenvalues are shown and discussed in a later section. There is little observed correlation between the accuracy of eigenvalues corresponding to symmetric eigenfunctions, i.e., the fundamental and every other eigenvalue thereafter, and those corresponding to asymmetric eigenfunctions, likely because the effect of the position and direction discretization on the calculated eigenvalues is greater. The eigenfunctions of the real eigenvalues are similar to the cosine shapes expected from basic diffusion theory, which follows $\cos (i \pi x / \Delta)$ where $i=1,2, \ldots$, defines the number of the eigenfunction.

For this scattering slab, the expanded flux solution accurately approximates the TDMC solution resulting from a initial pulse in the center of a slab (Figure 5.1). At $t=1$, neutrons are concentrated in the center of the slab as the move in all directions from the initial source. The expanded solution has a smooth, sharp decrease in the region ahead of the neutron pulse where there should be zero neutrons. This smooth shape is unable to approximate such a sudden change in the neutron flux. At $t=10$, neutrons nearly reach the boundaries of the slab, and the expanded flux solution only differs slightly to the TDMC solution at the front edge of the neutron pulse. At $t=20$, neutrons exit the slab and the smooth shapes of both the expanded flux TDMC solutions match. At $t=50$, the expanded flux solution takes the form of the fundamental mode, and decreases at the rate of the fundamental $\alpha$ eigenvalue. The 
Table 5.2: Comparison of TORTE- and GFM-calculated $\alpha$ eigenvalues for a homogeneous scattering slab.

\begin{tabular}{|c|c|c|c|}
\hline$\Delta$ & TORTE $^{\mathrm{a}, \mathrm{b}}$ & GFM & $\%$ relative error \\
\hline 1 & $-6.09822 \times 10^{-1}$ & $-6.08072 \times 10^{-1}$ & 0.288 \\
\hline 5 & $\begin{array}{l}-8.10462 \times 10^{-2} \\
-3.42167 \times 10^{-1} \\
-8.46262 \times 10^{-1}\end{array}$ & $\begin{array}{l}-8.10933 \times 10^{-2} \\
-3.41216 \times 10^{-1} \\
-8.34837 \times 10^{-1}\end{array}$ & $\begin{array}{l}0.0581 \\
0.279 \\
1.37\end{array}$ \\
\hline 10 & $\begin{array}{l}-2.53438 \times 10^{-2} \\
-1.04415 \times 10^{-1} \\
-2.40933 \times 10^{-1} \\
-4.40323 \times 10^{-1} \\
-7.04672 \times 10^{-1}\end{array}$ & $\begin{array}{l}-2.53500 \times 10^{-2} \\
-1.02978 \times 10^{-1} \\
-2.37942 \times 10^{-1} \\
-4.39813 \times 10^{-1} \\
-7.24185 \times 10^{-1}\end{array}$ & $\begin{array}{l}0.0245 \\
1.40 \\
1.26 \\
0.116 \\
2.69\end{array}$ \\
\hline 20 & $\begin{array}{c}-7.17976 \times 10^{-3} \\
-2.93535 \times 10^{-2} \\
-6.68243 \times 10^{-2} \\
-1.20005 \times 10^{-1} \\
-1.89650 \times 10^{-1} \\
-2.76313 \times 10^{-1} \\
-3.80676 \times 10^{-1} \\
-5.02925 \times 10^{-1} \\
-6.43331 \times 10^{-1} \\
-\end{array}$ & $\begin{array}{l}-7.17962 \times 10^{-3} \\
-2.91532 \times 10^{-2} \\
-6.62597 \times 10^{-2} \\
-1.19050 \times 10^{-1} \\
-1.88258 \times 10^{-1} \\
-2.74781 \times 10^{-1} \\
-3.79559 \times 10^{-1} \\
-5.03525 \times 10^{-1} \\
-6.52293 \times 10^{-1} \\
-8.43141 \times 10^{-1}\end{array}$ & $\begin{array}{l}0.00581 \\
1.07 \\
1.35 \\
1.38 \\
1.22 \\
0.861 \\
0.257 \\
0.661 \\
1.39 \\
-\end{array}$ \\
\hline 25 & $\begin{array}{c}-4.7169 \times 10^{-3} \\
-1.9369 \times 10^{-2} \\
-4.4073 \times 10^{-2} \\
-7.9002 \times 10^{-2} \\
-1.2440 \times 10^{-1} \\
-1.8052 \times 10^{-1} \\
-2.4763 \times 10^{-1} \\
-3.2595 \times 10^{-1} \\
-4.1549 \times 10^{-1} \\
-5.1588 \times 10^{-1} \\
-6.2665 \times 10^{-1} \\
- \\
-\end{array}$ & $\begin{array}{l}-4.71722 \times 10^{-3} \\
-1.92125 \times 10^{-2} \\
-4.36235 \times 10^{-2} \\
-7.81732 \times 10^{-2} \\
-1.23148 \times 10^{-1} \\
-1.78906 \times 10^{-1} \\
-2.45830 \times 10^{-1} \\
-3.24297 \times 10^{-1} \\
-4.14606 \times 10^{-1} \\
-5.17041 \times 10^{-1} \\
-6.34446 \times 10^{-1} \\
-7.91043 \times 10^{-1} \\
-9.55902 \times 10^{-1}\end{array}$ & $\begin{array}{l}0.00675 \\
0.808 \\
1.02 \\
1.05 \\
1.01 \\
0.894 \\
0.727 \\
0.507 \\
0.213 \\
0.225 \\
1.24 \\
- \\
-\end{array}$ \\
\hline
\end{tabular}

${ }^{\mathrm{a}}$ For $\Delta=1,5,10: N=201, M=16,100 \times 10^{6}$ active histories

${ }^{\mathrm{b}}$ For $\Delta=20,25$ : $N=400, M=8,200 \times 10^{6}$ active histories 
slight differences between the TDMC and expanded flux solutions at the edges of the propagating flux shape for early times is due to the difference between the initial sources defined in the TORTE eigenfunction expansion and TDMC calculation.

In this expansion, the initial source is in the center of the slab and angularly
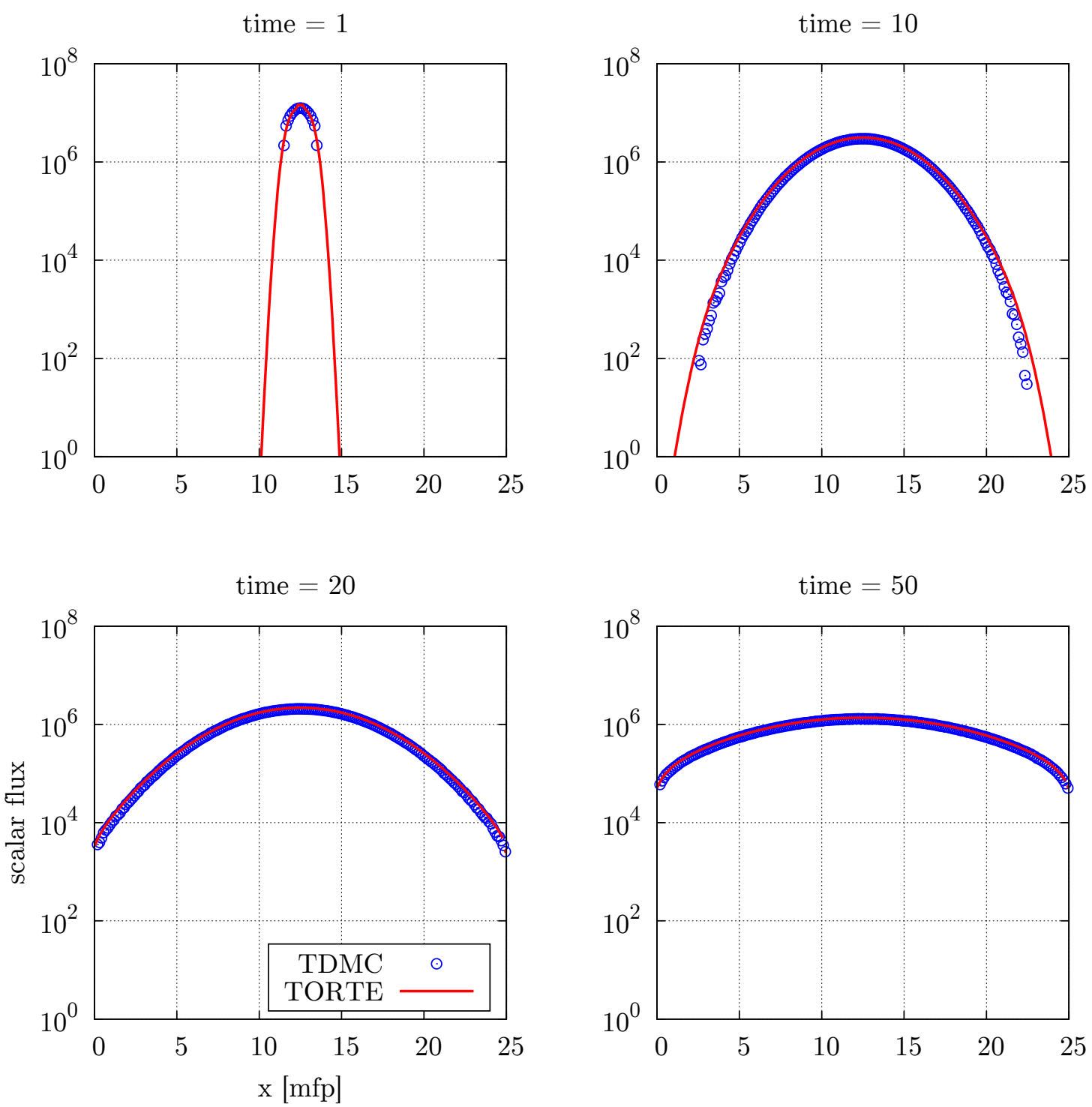

Figure 5.1: Snapshots compare the expanded flux solution to the TDMC solution, showing agreement throughout a transient in a scattering slab of thickness $\Delta=25.0 \mathrm{mfp}$. The TDMC solution is the result of tracking 100 batches of $1 \times 10^{6}$ neutron histories over $N=201$ position intervals. Each neutron history starts at $t=0$ at the exact the center of the slab $x=12.5$ moving in directions distributed equally in all $\mu$. TORTE uses $N=401$ position intervals and $M=8$ angular intervals so that the approximate source is in the center, slab $n=201$. The eigenfunction expansion uses all $N M$ modes, not just those corresponding to the real eigenvalues. 
symmetric, so the asymmetric, i.e., odd-numbered, kinetic modes are unimportant to the solution. This is preserved during the eigenfunction expansion, as the coefficients for the odd-numbered modes are several orders of magnitude smaller than coefficients of even-numbered ones: they contribute negligible amounts to the expanded flux solution.

Problems 10A-E: These are one-speed, multi-region slabs with two different material regions with $\Sigma_{t}=10$. Material 1 is purely-scattering, while material 2 is highly-scattering with $\Sigma_{s}=9$. The total problem thickness is $\Delta=10 \mathrm{mfp}$, the speed is effectively set to $v=10$, and the Corngold limit is $\alpha^{*}=-10$. Different divisions of the slab yields five separate variations, each with a different "grain size" defined as the thickness of a homogeneous subregion (Figure 5.2). In the limit of largest grain size, the problem has two grains, one on the left of material 1 and one on the right of material 2. In the opposite limiting case, the problem is a homogeneous slab with homogenized cross sections, $\Sigma_{s}=9.5$. For intermediate grain sizes, the materials alternate starting with material 1 until reaching the other end of the slab.

In all variations of this multi-region slab, the TORTE-calculated eigenvalues match the GFM eigenvalues within $0.2 \%$ (Table 5.3). The fundamental eigenvalues show the best agreement, within $0.06 \%$, while higher eigenvalues have the most difference. All eigenvalues converge within $0.2 \%$ regardless of the grain size and symmetric or asymmetric eigenfunctions. Like in the homogeneous slab, the shape eigenfunctions of the real eigenvalues behave as diffusion theory suggests, with additional inflections at each material interface.

The expanded flux solution for a pulse source incident on this multi-region slab matches TDMC solutions (Figure 5.3). At $t=0.5$, the neutrons reach the halfway point of the slab. Inflection points that mark the grain boundaries between material

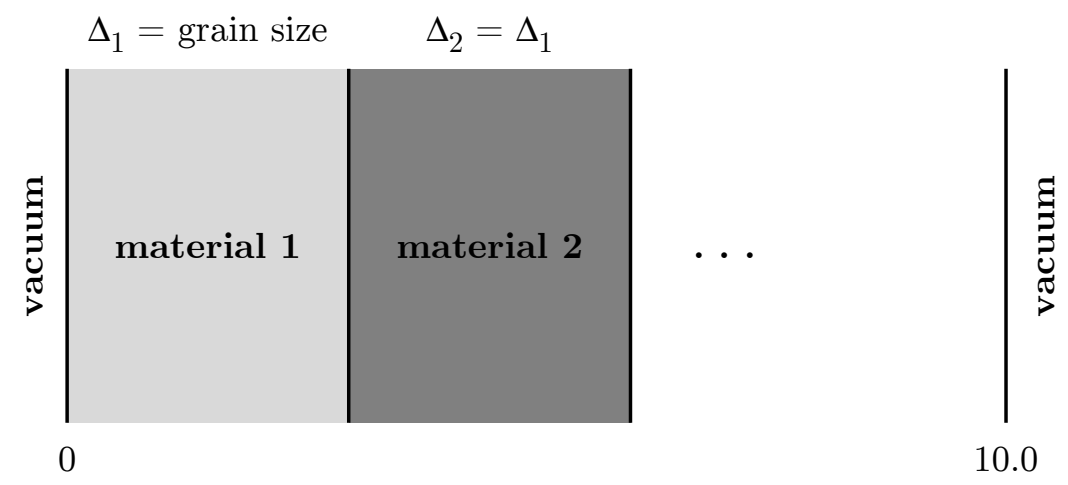

Figure 5.2: The multi-region slab consists of several subregions of alternating material. 
Table 5.3: TORTE- and GFM-calculated $\alpha$ eigenvalues for the multi-region slab.

\begin{tabular}{llll}
\hline Grain Size & TORTE $^{\mathrm{a}}$ & GFM & \% relative error \\
\hline 5.0 (2 slabs) & -0.550522 & -0.550812 & 0.0526 \\
& -1.70897 & -1.70645 & 0.148 \\
& -2.94639 & -2.94231 & 0.139 \\
& -5.15743 & -5.16275 & 0.103 \\
& -0.702916 & -0.703133 & 0.0309 \\
& -1.44948 & -1.44826 & 0.0842 \\
1.0 (10 slabs) & -3.07481 & -3.07065 & 0.135 \\
& -5.14246 & -5.14979 & 0.142 \\
& -0.748572 & -0.748792 & 0.0294 \\
& -1.55628 & -1.55474 & 0.0991 \\
& -2.96333 & -2.96021 & 0.105 \\
& -5.06238 & -5.06991 & 0.149 \\
& -0.756954 & -0.757198 & 0.0322 \\
& -1.56677 & -1.56505 & 0.120 \\
& -2.97862 & -2.97523 & 0.114 \\
& -5.09076 & -5.09813 & 0.145 \\
& -0.763313 & -0.763507 & 0.0254 \\
& -1.57345 & -1.57201 & 0.0916 \\
& -2.98655 & -2.98348 & 0.103 \\
& -5.10018 & -5.10866 & 0.166 \\
& & &
\end{tabular}

${ }^{\mathrm{a}} N=400, M=8,200 \times 10^{6}$ active histories

regions are present throughout the entire transient. The smooth expanded flux solution is not able to predict the steep drop-off at the front of the pulse at the center of the slab where zero neutrons exist. A slight bump in the expanded flux solution is different than the shape of the TDMC solution due to the differences in the initial source for the two cases. This difference is the direction dependence of neutrons within each direction interval: the TDMC solutions distributes source neutrons uniformly in $\mu$ over a given direction interval, but the rates in the TRM are generated with $\mu$ distributions that are likely not uniform across each direction interval. At $t=1.0$, neutrons reach the opposite side of the slab and begin to leak out of the boundary. Both solutions are smooth. At $t=1.5$, the asymmetric flux shape begins to level as more neutrons propagate to the right half of the slab. At $t=4.0$, all higher modes are nearly decayed as the expanded flux solution decreases significantly while approaching the fundamental kinetic mode.

Problems 9 and 10 demonstrate the extension of the TRMM to non-multiplying 
slabs. For large problems, finding the highest eigenvalues near the Corngold limit is the most difficult. The eigenfunction expansion delivers smooth functions which are unable to model very sharp changes in the TDMC solutions. Nevertheless, calculated eigenvalue spectra and expanded flux solutions compare well to benchmark results for both test problems.
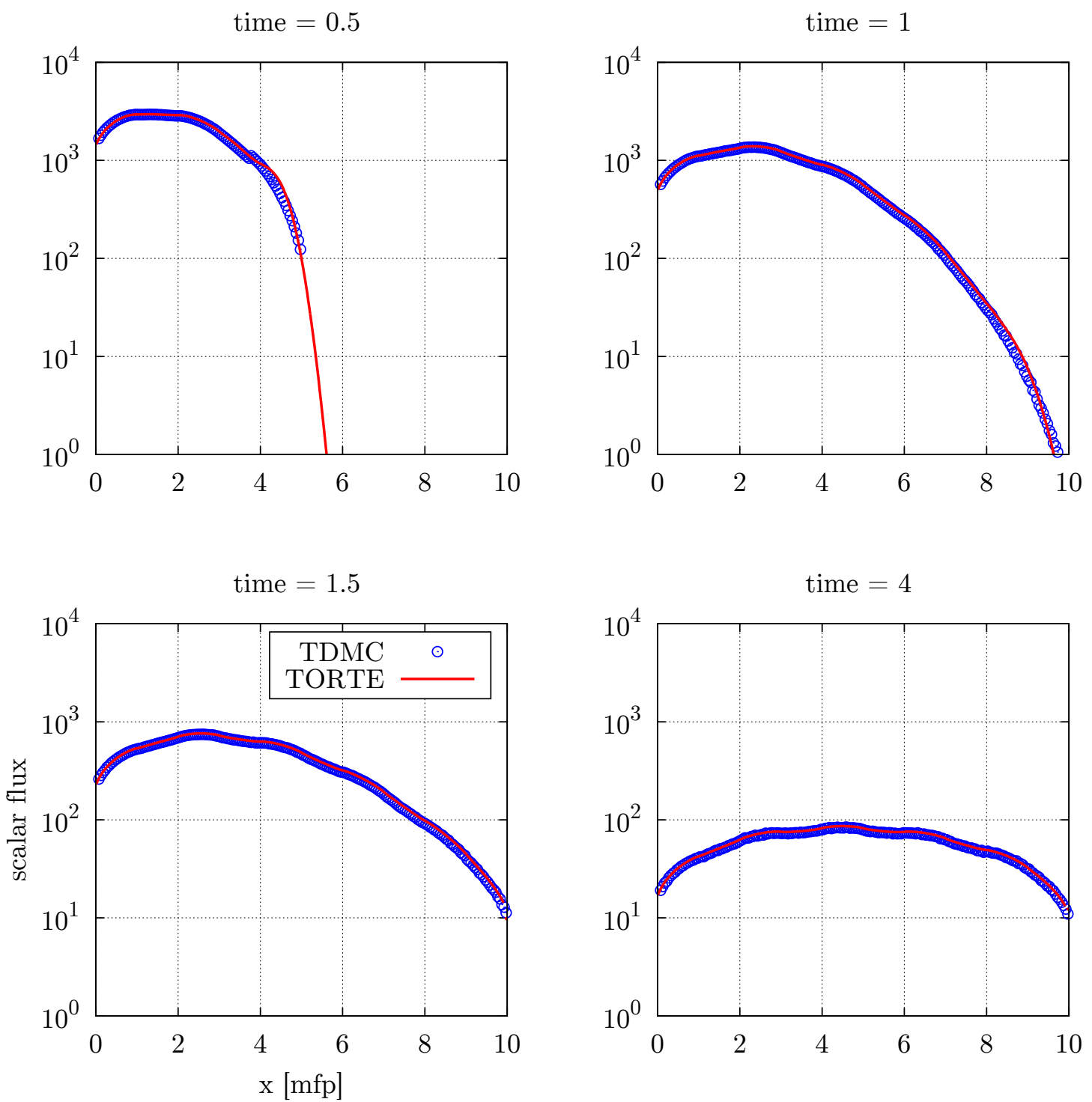

Figure 5.3: Snapshots comparing the expanded flux solution to the TDMC solution agree throughout a transient in a multi-region slab with ten homogeneous 1-mfp subregions of alternating materials. The TDMC solution is the result of tracking 100 batches of $1 \times 10^{6}$ neutron histories over $N=200$ position intervals. Neutron histories start at $t=0$ at the exact left edge of the slab $x=0$ distributed equally in $\mu$ between $0.75<\mu \leq 1.0$. TORTE approximates this source in the left-most slab $n=1$ and in the direction interval corresponding to the TDMC source, and uses all $N M$ modes in the eigenfunction expansion. 


\subsubsection{Multiplying Media}

Problems 11A-O: These are one-speed, homogeneous slabs of thicknesses ranging from $\Delta=1.0$ to $\Delta=50.0 \mathrm{mfp}$. The cross sections are $\Sigma_{s}=0.9$ and $\nu \Sigma_{f}=0.25$, and all absorptions are prompt fissions. The number of real $\alpha$ eigenvalues in the spectrum increases with the size of the slab, where for smaller slabs $\Delta<10.0$, there are no more than six real eigenvalues (Table 5.4). Larger slabs have up to thirty real eigenvalues (Table 5.5), with several positive eigenvalues. This increase is not a consequence of the phase space discretization: it is directly related to the increase in slab thickness. With a larger slab, more discrete eigenvalues are necessary to describe any asymmetry in the neutron flux.

For slab of all thicknesses, TORTE accurately obtains the fundamental and first few eigenvalues. For smaller slabs, the majority of the calculated eigenvalues fall within $1 \%$ of the GFM-calculated eigenvalues. Like the scattering slab problems, the eigenvalues closest to the Corngold limit are the most difficult to obtain, and in some cases, TORTE does not find the most negative eigenvalue in the spectrum. For example, for thicknesses of $\Delta=6,8$, and 10, the smallest GFM-calculated real eigenvalue is less than -0.80 , with the next smallest eigenvalue being nearly half that. TORTE does not calculate these eigenvalues close to the Corngold limit before the start of the complex portion of the eigenvalue spectrum. But, with an increase in the position and direction intervals, $N$ and $M$, TORTE will begin to deliver estimates of this eigenvalue. The TORTE-calculated eigenvalue closest to the limit is $\alpha=$ -0.63343 for the $\Delta=9$ slab, which matches well to the GFM-calculated eigenvalue.

Similar trends continue for the large slabs, for which TORTE uses a larger number of position and direction intervals. The fundamental and first few eigenvalues remain in agreement. But, as TORTE begins to calculate more real eigenvalues in the spectrum, they begin to differ more from the GFM-calculated spectrum. The size of the eigenvalue affects the accuracy, as those eigenvalues of very small magnitude, i.e., near zero, tend to differ more greatly from the GFM-calculated results than eigenvalues of larger magnitude. This is because the eigenvalues of the TRM are estimates of the true $\alpha$-eigenvalue spectrum, causing a larger relative shift for the smaller interior

eigenvalues. Again, TORTE is unable to calculate the highest eigenvalues close to the Corngold limit before the onset of the complex portion of the spectrum. For all slabs, there is little correlation between symmetric modes and accuracy of the calculated eigenvalue.

Problem 12: This is a two-region multiplying slab of thickness $\Delta=2.5 \mathrm{mfp}$. It consists of a 1.5-mfp region on the left with $\Sigma_{s}=0.9$ and $\nu \Sigma_{f}=0.6$, and a 1.0-mfp 
Table 5.4: Comparison of TORTE- and GFM-calculated $\alpha$ eigenvalues for small homogeneous multiplying slabs.

\begin{tabular}{|c|c|c|c|}
\hline$\Delta$ & TORTE $^{\mathrm{a}, \mathrm{b}}$ & GFM & $\%$ relative error \\
\hline 1 & -0.46979 & -0.46916 & 0.134 \\
\hline 2 & -0.13616 & -0.13631 & 0.110 \\
\hline 3 & $\begin{array}{l}-0.013949 \\
-0.56748\end{array}$ & $\begin{array}{l}-0.013979 \\
-0.56833\end{array}$ & $\begin{array}{l}0.215 \\
0.150\end{array}$ \\
\hline 4 & $\begin{array}{c}0.043690 \\
-0.30134\end{array}$ & $\begin{array}{c}0.043705 \\
-0.30054\end{array}$ & $\begin{array}{l}0.0343 \\
0.266\end{array}$ \\
\hline 5 & 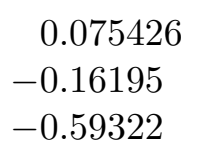 & $\begin{aligned} & 0.075469 \\
- & 0.16035 \\
- & 0.59898\end{aligned}$ & $\begin{array}{l}0.0570 \\
0.998 \\
0.962\end{array}$ \\
\hline 6 & $\begin{array}{c}0.094799 \\
-0.077252 \\
-0.38789 \\
-\end{array}$ & $\begin{aligned} & 0.094831 \\
- & 0.077292 \\
- & 0.38858 \\
- & 0.88268\end{aligned}$ & $\begin{array}{l}0.0336 \\
0.0516 \\
0.178 \\
-\end{array}$ \\
\hline 7 & $\begin{array}{c}0.10749 \\
-0.023985 \\
-0.25671 \\
-0.62459\end{array}$ & $\begin{array}{c}0.10751 \\
-0.023857 \\
-0.25690 \\
-0.61675\end{array}$ & $\begin{array}{l}0.0232 \\
0.537 \\
0.0733 \\
1.27\end{array}$ \\
\hline 8 & $\begin{array}{c}0.11624 \\
0.012395 \\
-0.16913 \\
-0.44186 \\
-\end{array}$ & $\begin{array}{c}0.11626 \\
0.012616 \\
-0.16883 \\
-0.44324 \\
-0.83417\end{array}$ & $\begin{array}{l}0.0191 \\
1.75 \\
0.180 \\
0.310 \\
-\end{array}$ \\
\hline 9 & $\begin{array}{c}0.12254 \\
0.038349 \\
-0.10750 \\
-0.32328 \\
-0.63343\end{array}$ & $\begin{array}{c}0.12256 \\
0.038649 \\
-0.10687 \\
-0.32364 \\
-0.62740\end{array}$ & $\begin{array}{l}0.0127 \\
0.775 \\
0.588 \\
0.111 \\
0.962\end{array}$ \\
\hline 10 & $\begin{array}{c}0.12722 \\
0.57586 \\
-0.062286 \\
-0.23795 \\
-0.47767 \\
-\end{array}$ & $\begin{array}{c}0.12725 \\
0.057894 \\
-0.061541 \\
-0.23748 \\
-0.48031 \\
-0.80528\end{array}$ & $\begin{array}{l}0.0225 \\
0.531 \\
1.21 \\
0.197 \\
0.550 \\
-\end{array}$ \\
\hline
\end{tabular}

${ }^{\mathrm{a}}$ For $\Delta=1,2,3,4,5: N=201, M=16,100 \times 10^{6}$ active histories

${ }^{\mathrm{b}}$ For $\Delta=6,7,8,9,10: N=400, M=8,200 \times 10^{6}$ active histories 
Table 5.5: Comparison of TORTE- and GFM-calculated $\alpha$ eigenvalues for large homogeneous multiplying slabs.

\begin{tabular}{|c|c|c|}
\hline$\Delta$ & TORTE $^{\mathrm{a}}$ & GFM \\
\hline 15 & $\begin{array}{l}0.13911,0.10585,0.049567 \\
-0.030957,-0.13718,-0.27101 \\
-0.43446,-0.64248\end{array}$ & $\begin{array}{l}0.13913,0.10626,0.050626 \\
-0.029113,-0.13499,-0.26990 \\
-0.43784,-0.64414,-0.89490\end{array}$ \\
\hline 20 & $\begin{array}{l}0.14364,0.12414,0.091285 \\
0.044738,-0.015984,-0.091470 \\
-0.18234,-0.28914,-0.41241 \\
-0.55442\end{array}$ & $\begin{array}{l}0.14365,0.12451,0.09233 \\
0.046662,-0.013142,-0.087970 \\
-0.17899,-0.28772,-0.41608 \\
-0.56654,-0.74205,-0.94561\end{array}$ \\
\hline 30 & $\begin{array}{l}0.14705,0.13793,0.12262, \\
0.10103,0.073081,0.038670, \\
0.0022682,-0.049900,-0.10432, \\
-0.16562,-0.23381,-0.30876 \\
-0.39052,-0.47898,-0.57630 \\
-0.71666\end{array}$ & $\begin{array}{l}0.14707,0.13825,0.12349, \\
0.10270,0.075746,0.042455, \\
0.0026071,-0.044071,-0.097908, \\
-0.15930,-0.22872,-0.30673, \\
-0.39400,-0.49130,-0.59956, \\
-0.71983,-0.85323\end{array}$ \\
\hline 40 & $\begin{array}{l}0.14829,0.14300,0.13410, \\
0.12153,0.10533,0.085480 \\
0.061859,0.034532,0.0034576, \\
-0.031412,-0.070027,-0.11252, \\
-0.15871,-0.20869,-0.26224 \\
-0.31925,-0.37966,-0.44327 \\
-0.51026,-0.58202,-0.66838\end{array}$ & $\begin{array}{l}0.14832,0.14326,0.13482, \\
0.12295,0.10763,0.088785, \\
0.066355,0.040254,0.010383, \\
-0.023376,-0.061157,-0.10312, \\
-0.14943,-0.20032,-0.25599, \\
-0.31673,-0.38283,-0.45465, \\
-0.53256,-0.61701,-0.70849, \\
-0.80753\end{array}$ \\
\hline 50 & $\begin{array}{l}0.14889,0.14541,0.13954, \\
0.13128,0.12065,0.10760, \\
0.092150,0.074257,0.053972, \\
0.031267,0.0061054,-0.021449 \\
-0.051437,-0.083819,-0.11851 \\
-0.15557,-0.19493,-0.23649 \\
-0.28012,-0.32571,-0.37322 \\
-0.42228,-0.47317,-0.52578 \\
-0.58126,-0.64332\end{array}$ & $\begin{array}{l}0.14891,0.14564,0.14018, \\
0.13251,0.12263,0.11050, \\
0.096098,0.079394,0.060343, \\
0.038900,0.015012,-0.011382, \\
-0.040349,-0.071968,-0.10632, \\
-0.14351,-0.18363,-0.22682, \\
-0.27319,-0.32289,-0.37610, \\
-0.43298,-0.49374,-0.55860, \\
-0.62780,-0.70162,-0.78033, \\
-0.86424,-0.95354\end{array}$ \\
\hline
\end{tabular}

${ }^{\mathrm{a}} N=400, M=8,200 \times 10^{6}$ active histories

region on the right with $\Sigma_{s}=0.2$ and $\nu \Sigma_{f}=0.3$. All absorptions are fissions.

The problem is supercritical with a TORTE-calculated $k$ eigenvalue of 1.286719 $\pm 11 \mathrm{pcm}$ and there is one real $\alpha$-eigenvalue in the spectrum. TORTE calculates this fundamental $\alpha$ eigenvalue as 0.14250191 , within $0.02 \%$ of the GFM-calculated eigenvalue, 0.14247481. Also, the GFM method provides normalized values of the 
fundamental shape eigenfunction calculated at specific points within the slab. This solution aligns with the TORTE-calculated fundamental shape eigenfunction (Figure 5.4 ), which has an inflection point at the material boundary $x=1.5$. TORTE calculates only this one real eigenvalue before the onset of the complex spectrum. Higher complex eigenfunctions have a similar behavior to the fundamental eigenfunction when plotting the real and imaginary parts separately in the position phase space.

\subsubsection{Mixed Media}

Problems 13A-D: These are variations of a five-region slab consisting of fuel, a moderator, and an absorber. The nuclear parameters for the fuel are either $\nu \Sigma_{f}=$ 0.3 or $=0.7$, and $\Sigma_{s}=0.8$, where all absorptions are fissions. The moderator is highly scattering with $\Sigma_{s}=0.8$ and the absorber has a small scattering cross section of $\Sigma_{s}=0.1$. The outer regions on left and right are fuel, the center region is a large absorber, and the regions in between are moderator (Figure 5.5). The thickness of the right fuel region $\Delta_{5}$ is either 1.0 or $1.1 \mathrm{mfp}$, creating symmetric and asymmetric

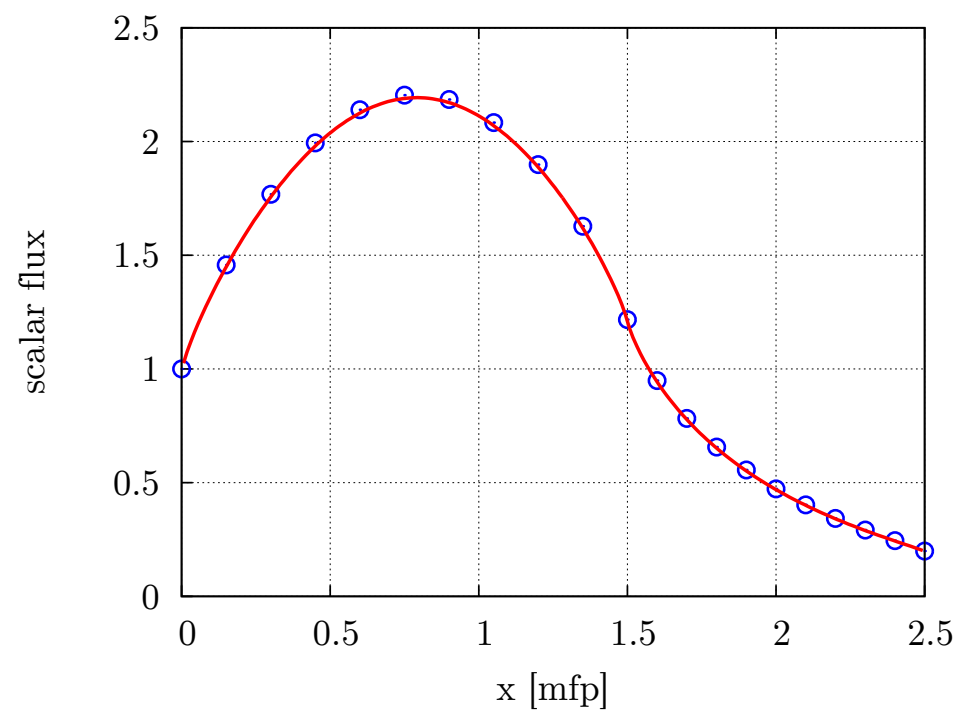

GFM $\odot$ TORTE

Figure 5.4: The TORTE-calculated fundamental shape eigenfunction of the two-region problem matches that calculated at exact points by the GFM. TORTE uses $N=200$ position and $M=8$ direction intervals, and runs $200 \times 10^{6}$ active histories. Note that the GFM calculates the scalar flux at $x=0$, while TORTE calculates the scalar flux within the small slab from $0 \leq x<\Delta_{n}$, where $\Delta_{n}$ is the thickness of the slab. This only causes a slight difference in the normalization procedure for the shape eigenfunction. 


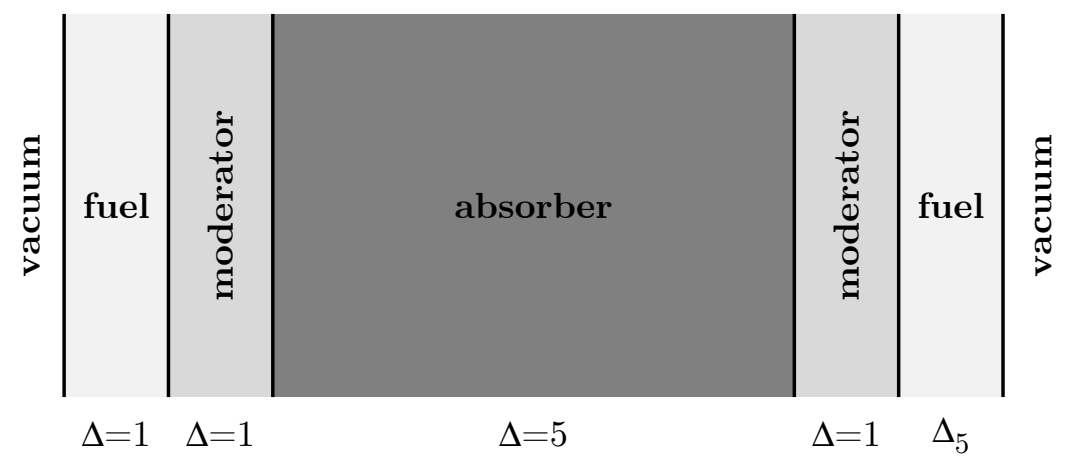

Figure 5.5: The five-region slab has loosely coupled fuel regions due to the large central absorber subregion.

configurations. With the variation on the fuel $\nu \Sigma_{f}$, there are four variants of this slab. These are the final problems in the GFM benchmark.

All variations of this slab have only two real $\alpha$ eigenvalues. The two fuel regions are loosely coupled due to the large absorber in between; very few neutrons born in one fuel region reach the other. Also, the first two $k$ eigenvalues and $\alpha$ eigenvalues are very close to one another. These factors slow the convergence rate, particularly in the configuration where the first two $\alpha$ eigenvalues are very small. TORTE calculates the fundamental $k$ eigenvalue (it does not calculate higher $k$ eigenvalues), and uses different numbers of position and direction intervals depending on the configuration.

Compared to the GFM-calculated eigenvalues, the TORTE-calculated $k$ eigenvalues are within one standard deviation and the $\alpha$ eigenvalues match to several significant digits (Table 5.6). The worst agreement is for the first $\alpha$ eigenvalue of the asymmetric $\nu \Sigma_{f}=0.7$ configuration, where the first two $\alpha$ eigenvalues differ more than in the other three configurations. TORTE has difficulty accurately obtaining this first eigenvalue, as it is an order of magnitude smaller than the fundamental. In all configurations, TORTE calculates only the two real eigenvalues before calculating complex spectra at and beyond the Corngold limit. Thus, TORTE obtains many more eigenvalues and shape eigenfunctions than the GFM.

The shape eigenfunctions corresponding to the two real eigenvalues of the $\nu \Sigma_{f}=$ 0.3 configurations (Figure 5.6) peak in the outlying fuel regions. The fundamental shape eigenfunctions are all positive and all real, while the first shape eigenfunctions have positive and negative parts. For the symmetric configuration, the absorber region causes an order of magnitude decrease in the center of the fundamental eigenfunction, while the first shape eigenfunction has two-fold rotational symmetry. With only a small increase in the size of the right fuel region, the fundamental shape 
Table 5.6: Summary of the TORTE and GFM results for the five-region slab.

\begin{tabular}{|c|c|c|c|c|c|c|}
\hline \multirow[b]{2}{*}{$n$} & \multirow[b]{2}{*}{$\nu \Sigma_{f}$} & \multirow[b]{2}{*}{$\Delta_{5}$} & \multirow{2}{*}{\multicolumn{2}{|c|}{$\begin{array}{c}k_{n} \\
\text { GFM }\end{array}$}} & \multicolumn{2}{|c|}{$\alpha_{n}$} \\
\hline & & & & & TORTE $^{\mathrm{b}, \mathrm{c}, \mathrm{d}, \mathrm{e}}$ & GFM \\
\hline 0 & 0.3 & 1.0 & 0.4243228 & 0.4243163 & -0.3196225 & -0.3196537 \\
\hline 1 & & & - & 0.4241317 & -0.3229678 & -0.3229855 \\
\hline 0 & & 1.1 & 0.4556890 & 0.4556758 & -0.2930021 & -0.2932468 \\
\hline 1 & & & - & 0.4242237 & -0.3211711 & -0.3213939 \\
\hline 0 & 0.7 & 1.0 & 0.9900525 & 0.9900716 & -0.006153130 & -0.006156369 \\
\hline 1 & & & - & 0.9896407 & -0.006442946 & -0.006440766 \\
\hline 0 & & 1.1 & 1.063237 & 1.063244 & 0.03753544 & 0.03759991 \\
\hline 1 & & & - & 0.9898554 & -0.006969051 & -0.006298843 \\
\hline & $\mathrm{a}$ & 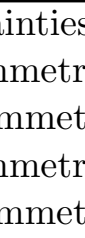 & verged to & $\begin{array}{l}\text { s than a f } \\
=180, M \\
\mathrm{~V}=182, M \\
=270, M \\
\mathrm{~V}=273, M\end{array}$ & $\begin{array}{l}\text { m. } \\
2 \times 10^{9} \text { active } \\
2 \times 10^{9} \text { active } \\
10 \times 10^{9} \text { active } \\
2 \times 10^{9} \text { active }\end{array}$ & $\begin{array}{l}\text { stories } \\
\text { iistories } \\
\text { iistories } \\
\text { iistories }\end{array}$ \\
\hline
\end{tabular}

eigenfunction increases an order of magnitude in the larger region. The first shape eigenfunction in the asymmetric configuration is only slightly negative in the larger fuel region. Features seen in the shape eigenfunctions of the $\nu \Sigma_{f}=0.7$ configurations are similar, but with the additional multiplication in the fuel regions, the behavior is amplified. No GFM shape eigenfunction data is available for these configurations.

For the $\nu \Sigma_{f}=0.7$ configuration, the TORTE-calculated shape eigenfunctions for the symmetric and asymmetric configurations match results from the GFM (Figure 5.7). TORTE has the most difficulty converging to the shape eigenfunctions for the symmetric configuration due to the very small absolute difference between the fundamental and first $\alpha$ eigenvalues, on the order of $10^{-4}$. Though TORTE converges to these eigenvalues after running a similar number of particles as the other configurations, it must run many more particles to obtain the appropriate shape eigenfunctions. While the fundamental and first $\alpha$ eigenvalues of the $\nu \Sigma_{f}=0.3$ symmetric configurations have a smaller relative difference, TORTE has much less difficulty converging to the appropriate symmetric eigenfunctions. There are two physical reasons for this slow convergence: the $\nu \Sigma_{f}=0.7$ symmetric configuration is very near critical, so the flux within the system decays slowly; and the two fuel regions are nearly decoupled. Thus, an asymmetric perturbation of the flux in this slab persists for long times before decaying to the symmetric solution: this happens only after the flux decreases several orders of magnitude. While the $\nu \Sigma_{f}=0.3$ configuration is similarly decoupled, it is very subcritical, so the flux decays quickly. The shape eigenfunctions of both sym- 


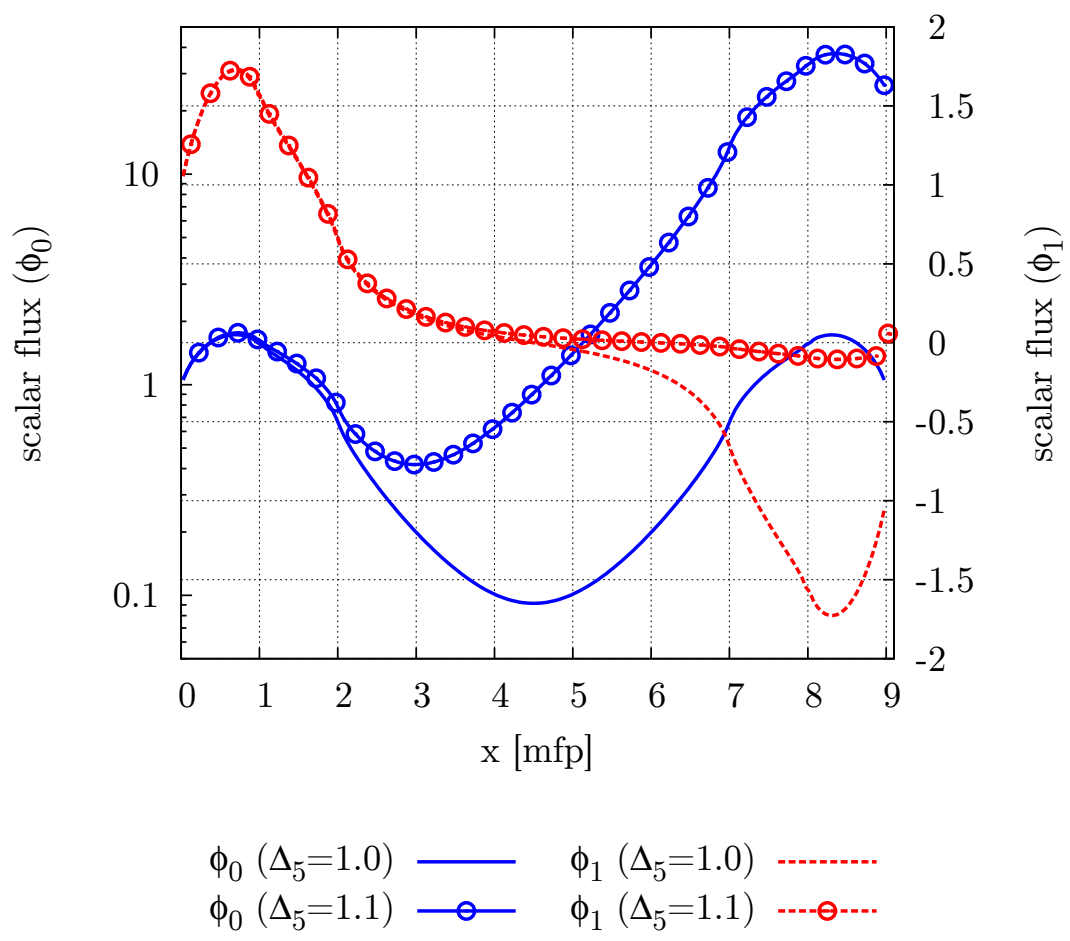

Figure 5.6: The TORTE-calculated first two real shape eigenfunctions of the $\nu \Sigma_{f}=0.3$ five-region slab show the different scales of the fundamental and first shape eigenfunctions and the large effect of a small increase in the right fuel region thickness.

metric configurations are similar, but the fundamental shape eigenfunction in this $\nu \Sigma_{f}=0.7$ configuration decreases two orders of magnitude in the central absorber region instead of only one.

The fundamental shape eigenfunction for the asymmetric $\nu \Sigma_{f}=0.7$ configuration increases three orders of magnitude in the larger right fuel region, a greater increase than in the $\nu \Sigma_{f}=0.3$ configuration. The first shape eigenfunctions of both configurations are similar. With an increase in size to the right fuel region, the subcritical symmetric slab becomes supercritical. This implies that the larger right fuel region drives the supercriticality of the slab, which is reflected in the increase of the fundamental eigenfunction.

For three configurations of the five-region slab, expanded flux solutions accurately predict the time-dependent evolution of the flux resulting from a rightward-directed initial source from the left vacuum. The eigenfunction expansions use all calculated modes, not only the two real modes. The selection of the source guarantees the excitation of asymmetric modes: a symmetric source is a simpler problem for the eigenfunction expansion. The TDMC solutions start initial neutrons distributed equally 

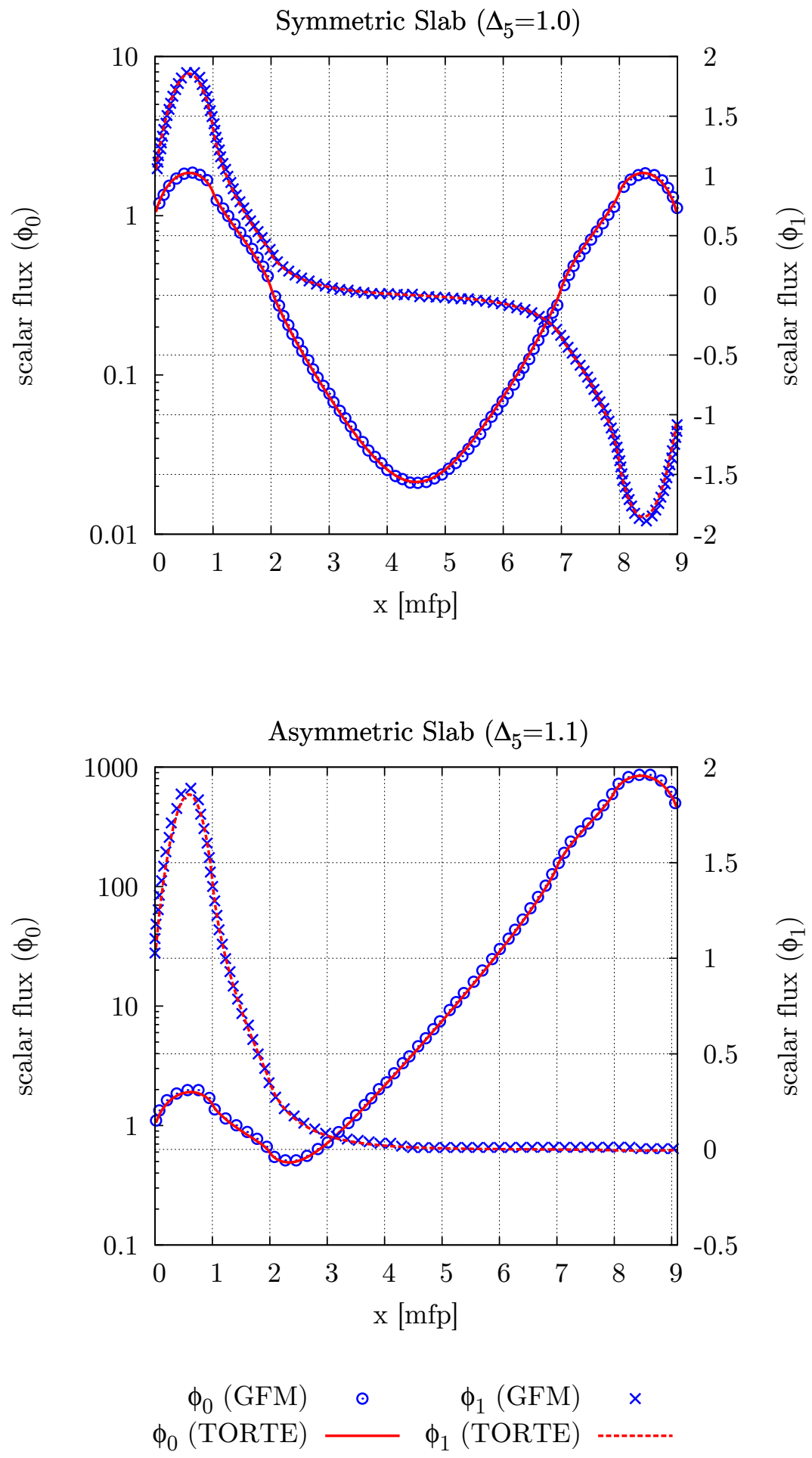

Figure 5.7: The TORTE-calculated first two real shape eigenfunctions of the symmetric and asymmetric $\nu \Sigma_{f}=0.7$ five-region slabs match those calculated by the GFM. 
between $0.75<\mu \leq 1.0$ to reflect the choice of $M=8$ equal-sized angular intervals and uses a coarser spatial resolution for increased statistics.

For an initial pulse on the slightly subcritical, symmetric $\nu \Sigma_{f}=0.7$ configuration, the behavior of the expanded flux solution throughout the transient is very smooth, though there are the usual issues at the edge of the propagating flux shape where the actual flux should drop abruptly to zero (Figure 5.8). At $t=4.5$, neutrons entering
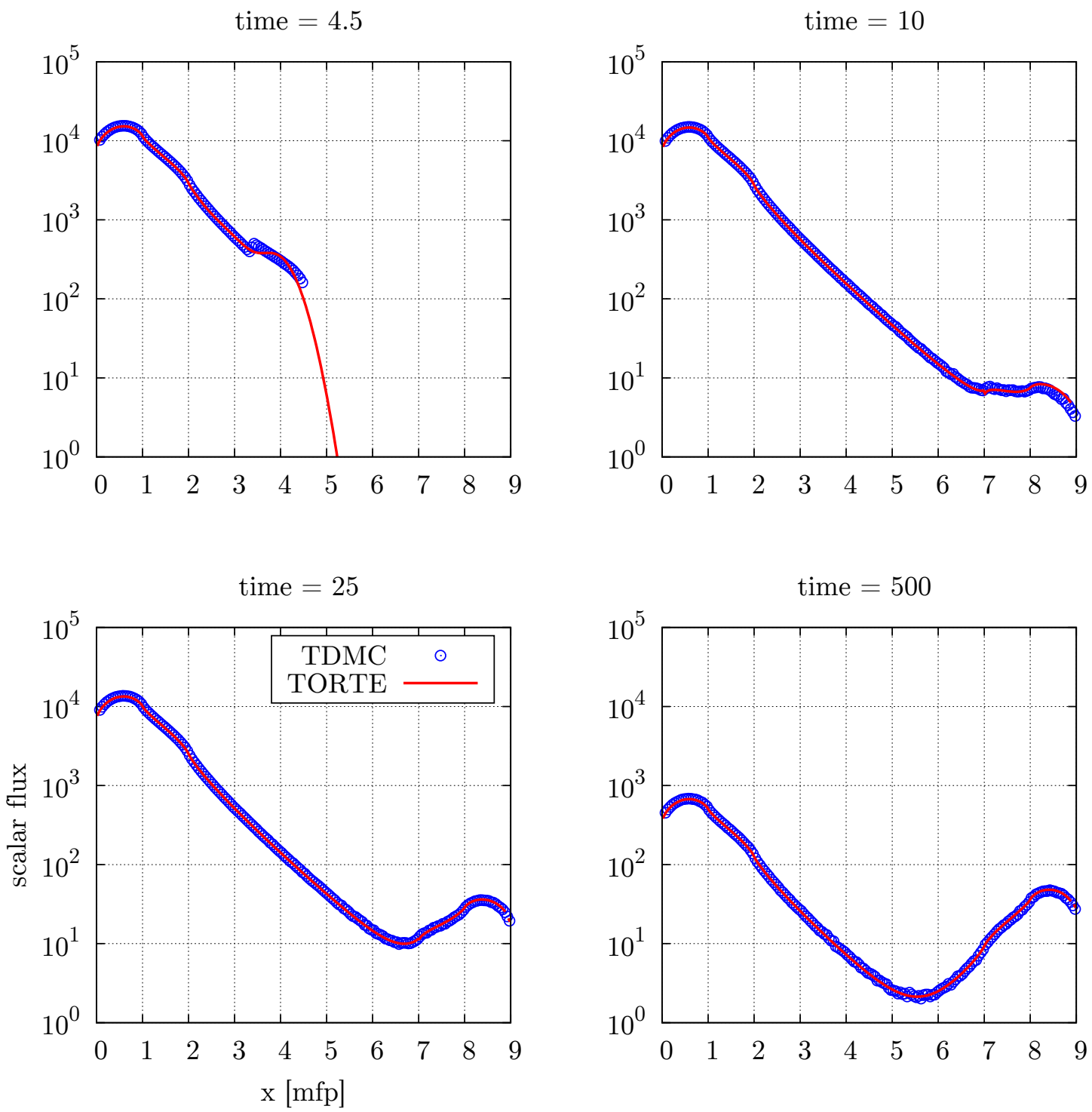

Figure 5.8: Snapshots of the expanded flux and TDMC solutions agree for a incident pulse on the subcritical, symmetric, $\nu \Sigma_{f}=0.7$ five-region slab. The TDMC solution is the result of tracking 100 batches of $1 \times 10^{6}$ neutron histories over $N=180$ position intervals. Each neutron history starts at $t=0$ at the left interface $x=0$. TORTE approximates this initial source as a pulse in the left slab $n=1$ and in the direction interval corresponding to the TDMC source. 
from the left penetrate to the center of the problem, where a small bump forms at the front of the distribution. The difference at this bump between the expanded flux and TDMC solutions is due to the slightly different definition of the sources, i.e., the difference between the uniform distribution of the TDMC source neutrons over each direction interval and the distribution used in calculating the rates of the TRM. Small inflections at $x=1$ and $x=2$ show the change in materials from fuel to moderator to absorber. There is a smooth drop off after $x=4.5$ in the expanded flux solution, where the TDMC solution abruptly ends. At $t=10$, the neutrons arrive at the other end of the slab and begin to fission in the right fuel region. There is a slight difference between the expanded flux and TDMC solutions at the right end of the slab, but the solutions match well despite the change in flux of over three orders of magnitude from the left to the right fuel. Inflection points show the change in materials at the right end of the slab. At $t=25$, the neutrons in the right fuel region continue to fission and the flux there recovers as more neutrons enter the fuel region. The flux at the left side of the slab changes very little during this time. At $t=500$, the flux in the right fuel region remains the same, while the flux on the left side of the slab decreases an order of magnitude. This behavior shows the decay of the first eigenfunction. After this time, the expanded flux solution continues to decrease as the asymmetry persists for a long time before approaching the symmetric fundamental mode.

An alternate way to visualize the expanded flux solution is to examine the timedependent variation of the flux at a certain point within the system. For the symmetric subcritical configuration (Figure 5.8), this is like the simulation of detector response in a pulsed-neutron experiment. Using this analysis, the expanded flux solution matches the TDMC solution for an ideal detector in the center of the right fuel region (Figure 5.9). The TDMC solution shows the effect of Monte Carlo statistics, where the solution disagrees slightly with the expanded flux solution at some times. The expanded flux solution yields values at any time after the pulse $t>0$, while the TDMC solution is limited to the initial definition of the census times.

Using the same initial source, but replacing the subcritical, symmetric slab with the asymmetric, supercritical slab and repeating the analysis delivers a similar expanded time-dependent solution. This solutions is identical to that of the subcritical slab until neutrons reach the right fuel region from the incident pulse at the left vacuum (Figure 5.10). The expanded flux and TDMC solutions show similar trends at $t=4.5$ and $t=9$ to the symmetric configuration. At $t=30$, the flux in the right fuel region recovers faster than in the symmetric configuration. The additional $0.1 \mathrm{mfp}$ provides enough fissile material to make this configuration supercritical. At $t=200$, 


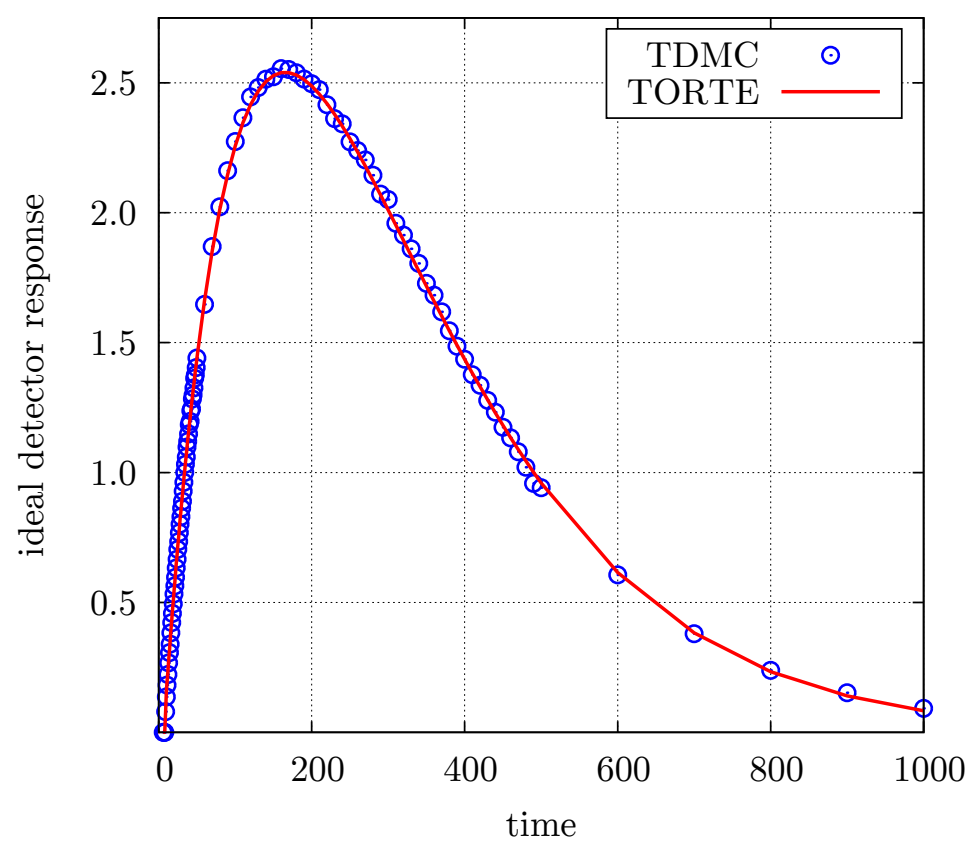

Figure 5.9: An alternate visualization of the expanded flux solution shows agreement with the TDMC solution for the ideal detector response at a point in the right fuel region. Because the TDMC and TORTE solutions use $N=180$ and $N=270$ position intervals, respectively, the slabs $n=169,170$ and $n=253,254$, and 255 represent the same position range. This represents the integrated scalar flux in the range $8.4<x<8.5$. The irregular frequency of the TDMC solution is due to the scaling of census times.

the flux in the right fuel region increases three orders of magnitude, as the flux in the left fuel region actually decreases. This decrease shows the decay of the first kinetic mode: the first eigenfunction is peaked in the left fuel region. Physically, the flux decreases on the left because there are too few fissions in the location to counterbalance the leakage of neutrons: this is the case for the symmetric slab. Thus, the flux in the left fuel region decreases until neutrons emitted from fission in the right fuel region begin to offset the imbalance in the left fuel region. The flux continues to increase rapidly before assuming the very asymmetric shape of the fundamental mode. The expanded flux solution accurately matches the TDMC solution throughout the transient.

The very subcritical, symmetric $\nu \Sigma_{f}=0.3$ configuration has a similar timedependent solution as the $\nu \Sigma_{f}=0.7$ configuration, except the solution decays much faster. But, if the initial source is constant for $t \geq 0$ with no initial neutrons within the problem, the expanded flux and TDMC solutions take on a much different form than the fundamental eigenfunction suggests (Figure 5.11). At $t=4.5$, neutrons reach the center of the problem, where the flux decreases several orders of magni- 
tude. A slight discrepancy appears between the expanded and TDMC solutions at the center. At $t=9$, very few neutrons reach the right edge of the problem. There is more statistical noise in the TDMC solution on the right half of the slab due to the low probability of neutrons traveling through the central absorber. At $t=12$, the flux in the right fuel region begins to increase from fissions in the fuel, but the flux solution is still very asymmetric. At $t=25$, the flux reaches its asymptotic solution:
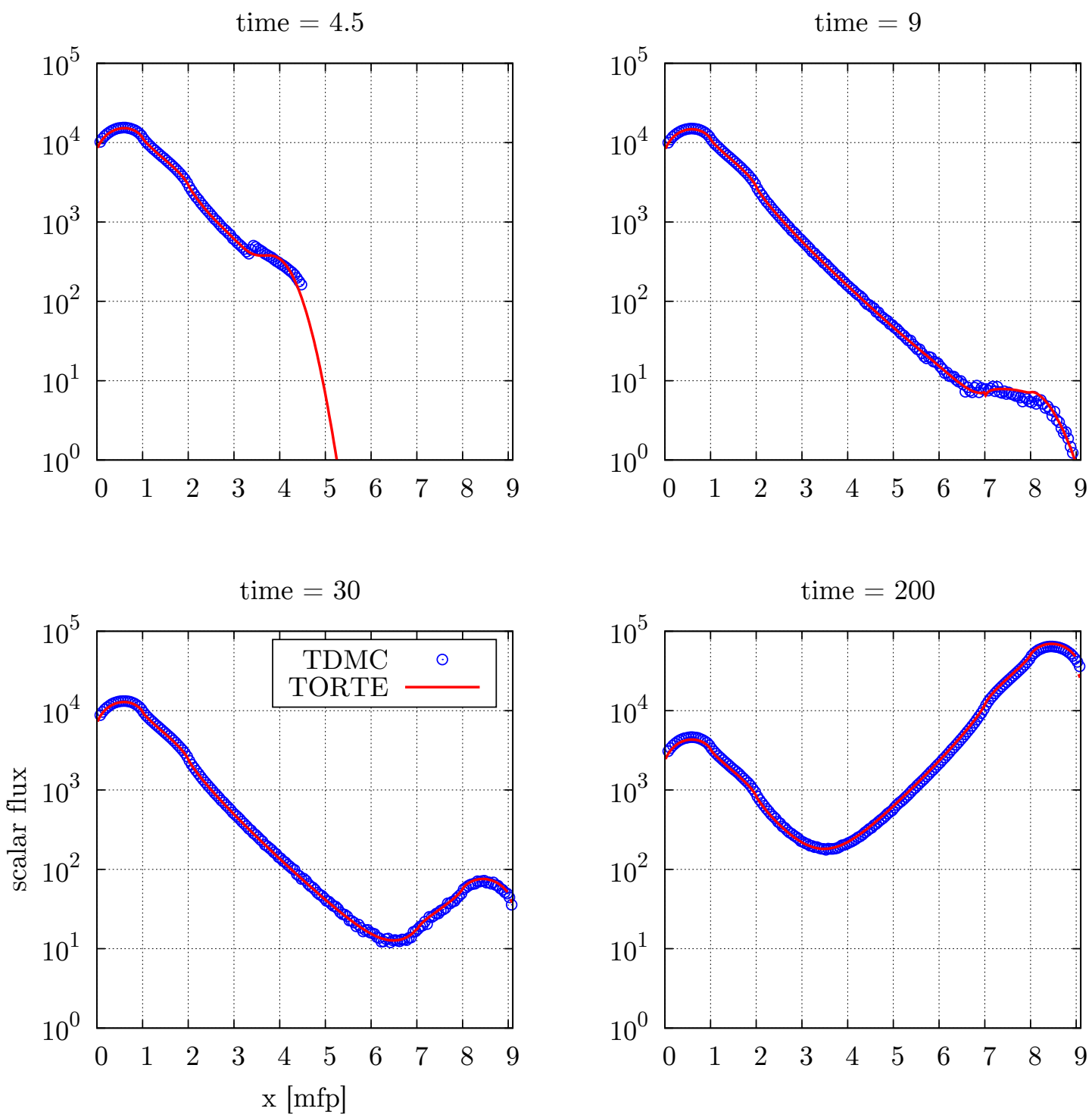

Figure 5.10: Snapshots of the expanded flux and TDMC solutions agree for an incident pulse on the supercritical, asymmetric, $\nu \Sigma_{f}=0.7$ five-region slab. The TDMC solution is the result of tracking 1,000 batches of 10,000 neutron histories over $N=182$ position intervals. Each neutron history starts at $t=0$ at the left interface $x=0$. TORTE approximates this initial source as a pulse in the left slab $n=1$ and in the direction interval corresponding to the TDMC source. 
all higher kinetic modes decayed and the asymmetric shape is due to the constant source entering from the left vacuum. While there is still noise in the TDMC solution in the right fuel region, the two solutions agree on this steady-state shape.

Problems 14A\&B: These are a variation of the symmetric configurations of the five-region slab, where the central absorber is replaced with the moderator material.
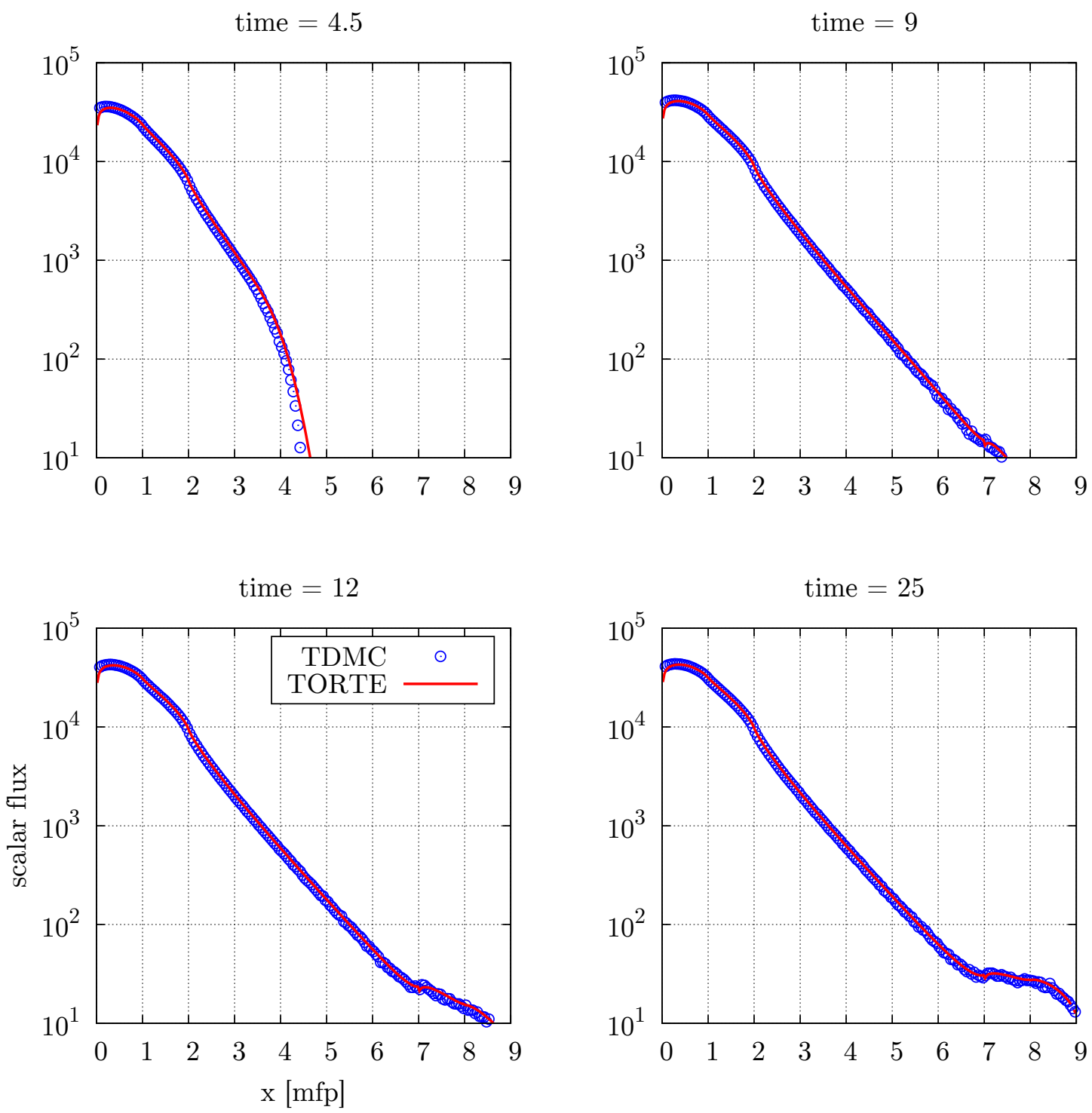

Figure 5.11: Snapshots of the expanded flux and TDMC solutions agree for a constant incident source on the very subcritical, symmetric, $\nu \Sigma_{f}=0.3$ five-region slab. The TDMC solution is the result of tracking 1,000 batches of 10,000 neutron histories over $N=180$ position intervals Neutron histories start at the left interface $x=0$, and initial start times are distributed evenly throughout the time before the last census. TORTE approximates this initial source as a constant source in the left slab $n=1$ and in the direction interval corresponding to the TDMC source. 
With this material change, TORTE calculates two additional real $\alpha$ eigenvalues (Table $5.7)$ before the start of the complex spectrum near the Corngold limit. There are no benchmark results for comparisons of these calculated eigenvalues. In the $\nu \Sigma=0.7$ configuration, the replacement of the absorber yields a supercritical configuration with two positive eigenvalues: an asymmetry in the flux affects the time-dependent solutions for long times. The asymptotic solution still follows the fundamental kinetic mode because it dominates the first kinetic mode at long times. The additional higher eigenfunctions cross the $x$-axis $n$ times, behaving similarly to the higher eigenfunctions in the five-region slab.

Table 5.7: The TORTE-calculated eigenvalues of the five-region slab with a central moderator replacing the absorber.

\begin{tabular}{|c|c|c|c|c|}
\hline \multirow[b]{2}{*}{$n$} & \multicolumn{2}{|c|}{$\nu \Sigma_{f}=0.3$} & \multicolumn{2}{|c|}{$\nu \Sigma_{f}=0.7$} \\
\hline & $k_{n}^{\mathrm{a}}$ & $\alpha_{n}^{\mathrm{b}}$ & $k_{n}^{\mathrm{a}}$ & $\alpha_{n}^{\mathrm{b}}$ \\
\hline 0 & 0.43720 & -0.20075 & 1.02013 & 0.010844 \\
\hline 1 & - & -0.25134 & - & 0.0083481 \\
\hline 2 & - & -0.40970 & - & -0.30124 \\
\hline 3 & - & -0.71076 & - & -0.57557 \\
\hline
\end{tabular}

${ }^{a}$ Uncertainties converged to less than a few pcm.

b $N=270, M=8,2 \times 10^{9}$ active histories

Problems 15A\&B: These are another variation of the symmetric configurations of the five-region slab, where the central absorber is replaced with a void: neutrons stream freely to the opposite side of the slab. For the $\nu \Sigma_{f}=0.7$ configuration, the removal of the central absorber makes the configuration supercritical. While TORTE still calculates a real $\alpha$ eigenvalue larger than other eigenvalues in the spectrum (Table 5.8 ), the presence of complex eigenvalues with real parts very near that of the largest eigenvalue makes identifying this as the true fundamental eigenvalue difficult. This is due to the central void region, where $\Sigma_{t}=0.0$ : this effectively moves the Corngold limit to $\alpha^{*}=0$ for the void, and TORTE calculates complex eigenvalues in the entire negative half space. In the $\nu \Sigma_{f}=0.7$ configuration, this is not a problem because the configuration is supercritical: the fundamental eigenvalue is positive and exists above this Corngold limit. But, in the subcritical $\nu \Sigma_{f}=0.3$ configuration, the fundamental $\alpha$ eigenvalue is negative and the calculation of complex spectra in the space around the fundamental eigenvalue makes it difficult to identify, and it moves significantly depending on the position and direction phase space discretizations. Using more position and direction intervals improves the accuracy the expanded time-dependent solution, but the eigenvalues calculated so close to the fundamental will always affect 
Table 5.8: The largest TORTE-calculated $\alpha$ eigenvalues of the five-region slab with a central void replacing the absorber.

\begin{tabular}{ccccc}
\hline \multirow{2}{*}{$M$} & \multicolumn{2}{c}{$\nu \Sigma_{f}=0.3$} & \multicolumn{2}{c}{$\nu \Sigma_{f}=0.7$} \\
& $k_{0}^{\mathrm{a}}$ & $\alpha_{0}^{\mathrm{b}}$ & $k_{0}^{\mathrm{a}}$ & $\alpha_{0}^{\mathrm{b}}$ \\
\hline 8 & \multirow{2}{*}{0.47544} & -0.049392 & \multirow{2}{*}{1.10938} & 0.035506 \\
30 & & -0.026828 & & 0.035605 \\
\hline
\end{tabular}

${ }^{\mathrm{a}}$ Uncertainties converged to less than a few pcm.

b $N=270,2 \times 10^{9}$ active histories, same random number seed.

this eigenvalue and its shape eigenfunction.

Like in the expanded flux solution of the unphysical, 81-group infinite medium, the calculation of complex eigenvalues so close to the fundamental causes long-lived oscillations. But, the expanded flux solution oscillates towards the end of the transient instead of at the beginning (Figure 5.12). Reacting to a pulsed source, the expanded flux and TDMC solutions are in agreement for early times. At $t=4.5$, neutrons reach the center of the void, where the expanded flux solution has some issues obtaining the shape at the edge of the propagating pulse. At $t=10$, neutrons enter the right fuel region and fission, causing a slight recovery of the flux there. The expanded flux solution eventually reaches a smooth, symmetric shape, until neutrons from the right fuel region return to the left fuel region. At $t=45$, unphysical oscillations that are not in the TDMC solution begin to appear in the expanded flux solution. The amplitude of these oscillations increases as the flux solution continues to decay. At $t=100$, the TDMC solution is nearly decayed and the oscillation in the expanded flux solution causes significant deviations. Oscillations in the expanded flux solution persist until $t=1500$, at which point the eigenfunction corresponding to the largest eigenvalue dictates the shape of the solution. The expanded flux solution is nearly zero at this time.

There are no oscillations in the expanded flux solution for the supercritical slab because the increasing fundamental kinetic mode dominates contributions of the higher modes: in the subcritical configuration, oscillations only appear after the solution decays several orders of magnitude. Oscillations only occur in central void, but effect the flux value at the void-moderator interface: the solutions within the fuel and moderator regions match well to the TDMC solution throughout the transient. This extends to the asymptotic flux solution, which does not oscillate, but has an odd, concave shape in the central void region. From these observations, it is easy to conclude that the middle $N=150$ position intervals consisting of void cause problems in the TRMM calculation: the TRMM does not apply well to media with large voids, 
especially when several position intervals are defined over this void. Still, this residual term is very small and only affects the expanded solution after it has decayed several orders of magnitude in the subcritical case. This behavior is empirical evidence that the separability of the time-dependence from the rest of the phase space in the neutron transport equation is invalid for streaming media, as expected.
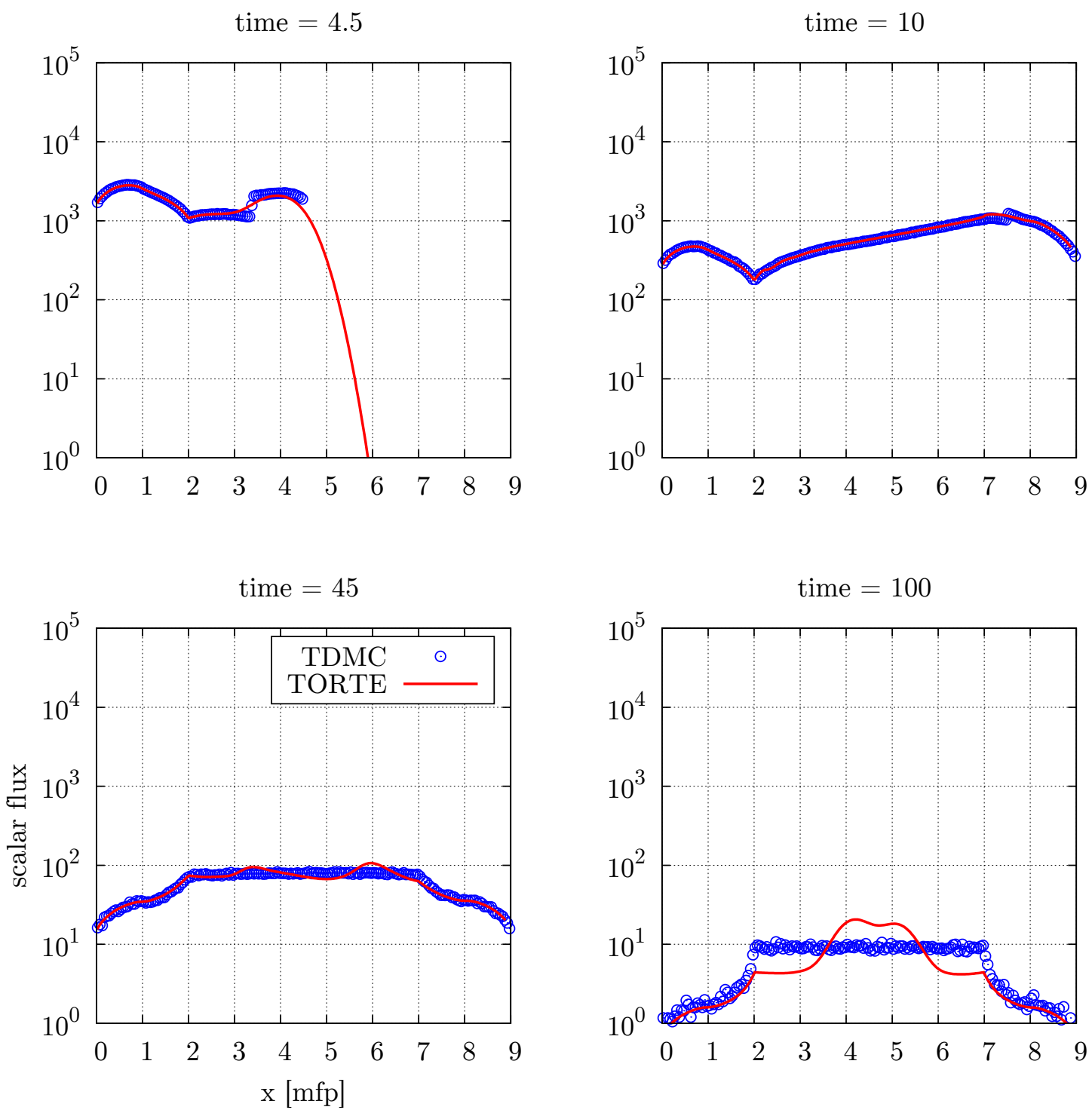

Figure 5.12: Snapshots of the expanded flux and TDMC solutions agree until oscillations appear at later times for a incident pulse on the very subcritical, symmetric, $\nu \Sigma_{f}=0.3$ five-region slab with a central void region. The TDMC solution is the result of tracking 1,000 batches of 10,000 neutron histories over $N=180$ position intervals. Each neutron history starts at $t=0$ at the left interface $x=0$. TORTE uses $M=30$ direction intervals, approximating the initial source as a pulse in the left slab $n=1$, where the source directions are distributed over the four intervals closest to $\mu=+1$. 


\subsubsection{Position and Direction Intervals}

To investigate the effect that discretizing the position-direction phase space has on calculated $\alpha$-eigenvalue spectra, TORTE runs the homogeneous, purely-scattering, $\Delta=5.0 \mathrm{mfp}$ thick slab with different numbers of position and direction intervals $N$ and $M$. Theoretically, the spectrum of this simple slab consists of three real eigenvalues and the continuum beyond the Corngold limit $\alpha^{*}=-1$, where no eigenvalues exist. TORTE calculates these real eigenvalues, but also calculates an additional spectrum of $N M-3$ eigenvalues. Most of these eigenvalues fall into the continuum, making up a large, circular spectrum whose approximate overall radius depends on the number of position intervals TORTE uses (Figure 5.13). The real eigenvalues exist on the right end of the spectrum and are lost in a plot of all calculated eigenvalues. With a two-fold increase in $N$, the approximate radius of the spectrum doubles. The physical reason for this is that events occur more rapidly in a system with large $N$ because the individual position intervals are smaller. The rates in the TRM become smaller, and transfers between states occur more rapidly than with smaller $N$. This necessitates eigenvalues with a greater negative magnitude. This trend is witnessed for position interval numbers ranging from $N=50$ to $N=500$.

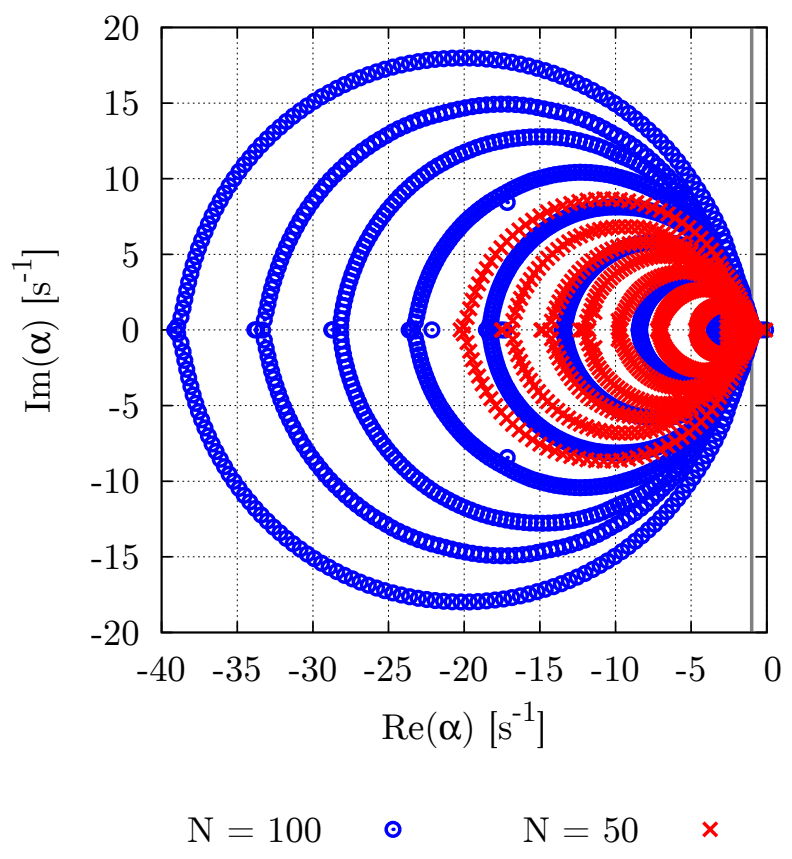

Figure 5.13: The size of the circular shape of the TORTE-calculated $\alpha$-eigenvalue spectrum of the $\Delta=5.0 \mathrm{mfp}$ thick scattering slab increases with the number of position intervals. TORTE uses $M=16$ direction intervals and $200 \times 10^{6}$ active histories, and the bold line denotes the Corngold limit. 
Within the circular eigenvalue spectrum, eigenvalues align along distinct rings of different radii starting near the Corngold limit and extending into the continuum. The number of rings is equal to half the number of direction intervals TORTE uses (Figure 5.14). With only $M=2$ direction intervals, the single calculated ring is only half the size of the total extent of the spectrum. As $M$ increases, the rings fill out the extent of the spectrum. Each ring of eigenvalues corresponds to a pair of direction intervals symmetric across $\mu=0$. The smallest rings correspond to the direction intervals closest to $\mu=0$, the direction parallel to the slab faces. Likewise, the largest rings correspond to the direction intervals closes to $\mu=-1$ and $\mu=+1$. The physical reason for this is that neutrons traveling close to the $\mu=0$ direction stream for long times before experiencing an event. Thus, the rates associated with these neutrons are smaller than neutrons traveling in a direction perpendicular to the slab faces. Using an odd $M$ causes eigenvalues to flood the real axis: these additional all-real eigenvalues form a line extending from the Corngold limit in the negative direction. These eigenvalues are observed to interfere more with the calculated real, discrete $\alpha$ eigenvalues than using even $M$ : this is why the TRMM uses an even $M$ in all calculations. As $M$ continues to increase, the rings of eigenvalues begin to fill the extent of the spectrum entirely (Figure 5.15).

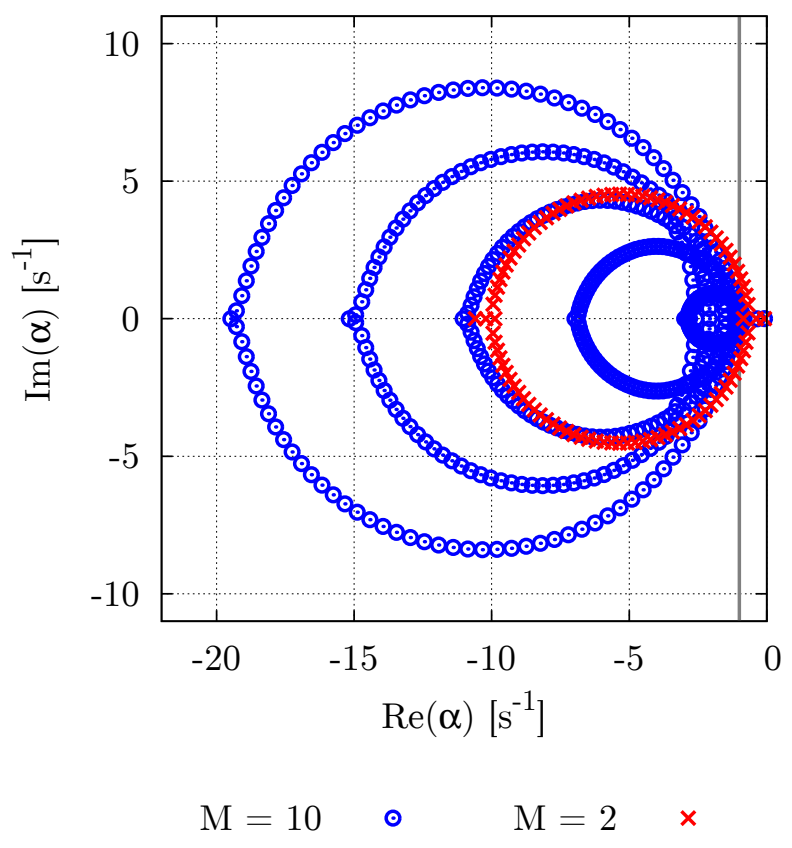

Figure 5.14: There are $M / 2$ rings of eigenvalues in the TORTE-calculated $\alpha$-eigenvalue spectra of the $\Delta=5.0 \mathrm{mfp}$ thick scattering slab. TORTE uses $N=50$ position intervals and $200 \times 10^{6}$ active histories, and the bold line denotes the Corngold limit. 


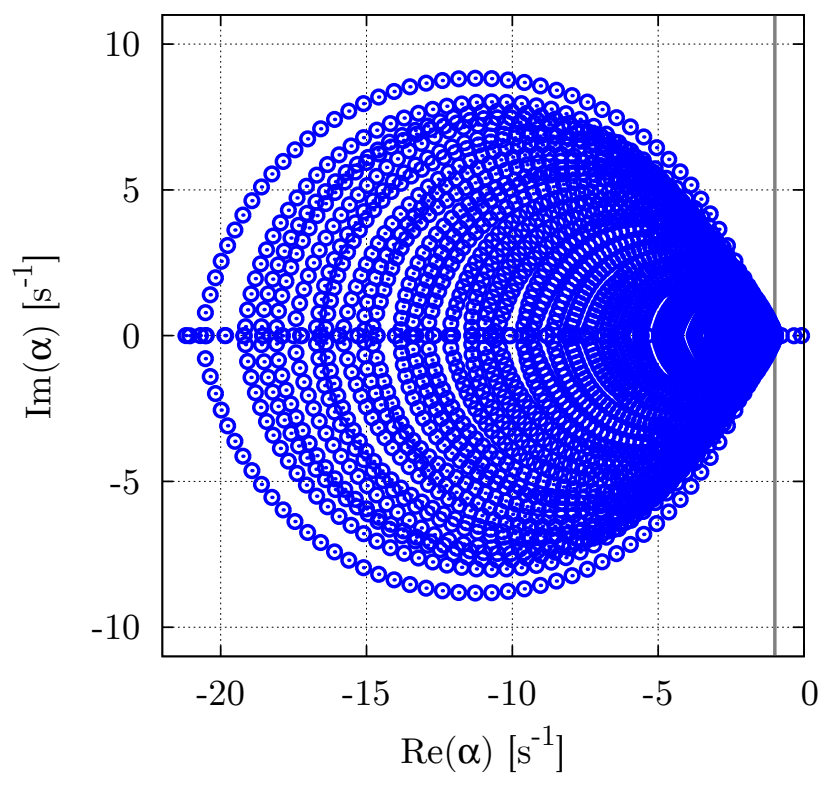

Figure 5.15: As the number of direction intervals increases to $M=64$, TORTE calculates additional rings of $\alpha$ eigenvalues that fill in a circle limited by the number of position intervals. This spectrum contains 32 distinct rings of eigenvalues. TORTE uses $N=50$ position intervals and $200 \times 10^{6}$ active histories for the $\Delta=5.0 \mathrm{mfp}$ thick scattering slab, and the bold line denotes the Corngold limit.

This behavior of the TORTE-calculated $\alpha$ eigenvalue spectrum suggests two trends: (i) in the limit of continuous direction intervals, the circular extent of the spectrum is completely populated with eigenvalues; and (ii) in the limit of continuous position intervals, the extent of the spectrum has an infinite radius. In these limits, the eigenvalues form the continuum, as expected from theory. For finite numbers of position and direction intervals, TORTE approximates the full spectrum, i.e., the discrete eigenvalues plus the continuum, by calculating the real eigenvalues above the Corngold limit and additional eigenvalues beyond the Corngold limit, whose positioning in the complex space is entirely dependent on the discretization of the position-direction phase space. This is similar to the way TORTE approximates continuous-energy infinite-media spectra with $G$ energy intervals.

In approximating the full spectrum, TORTE actually calculates more eigenvalues within the real portion of the spectrum than just the discrete eigenvalues. These complex eigenvalues are very near the Corngold limit, and with an increase in the number of position and direction intervals, the eigenvalues begin to disappear back into the continuum. Similar behavior is seen in some other calculated $\alpha$-eigenvalue 
spectra using matrix methods [5]. This suggests that TORTE has some difficulty approximating the continuum and a few discrete eigenvalues correctly, and has some difficulty limiting excess eigenvalues to the region $\alpha^{*}=-1$. This has two effects on calculated spectra: (i) when there are more discrete eigenvalues in the spectrum, TORTE finds better estimates of the fundamental and first few eigenvalues because they are less affected by the eigenvalues calculated in the continuum; (ii) if the last discrete eigenvalue is near the Corngold limit, the calculated higher spectrum may interfere and make it difficult to find this eigenvalue.

While the number of position and directions intervals greatly affects the overall behavior of the eigenvalue spectrum, it has relatively smaller effects on the discrete, real eigenvalues (Figure 5.16). For the same $M$, the fundamental $\alpha$ eigenvalue changes less than $0.25 \%$ as $N$ increases from $N=50$ to $N=500$, and stops trending near $N=300$. The first eigenvalue changes up to $1.0 \%$ over the same increase, and still shows some trend near $N=500$. An increase in $M$ has a much smaller effect on the calculated discrete eigenvalues than an increase in $N$ (Figure 5.17). For the same $N$, the fundamental $\alpha$ eigenvalues changes less than $0.15 \%$ as $M$ increases from $M=4$ to $M=64$, and stops trending near $M=20$. The first eigenvalue changes up to $0.25 \%$ over the same increase, but trends very little after $M=40$.

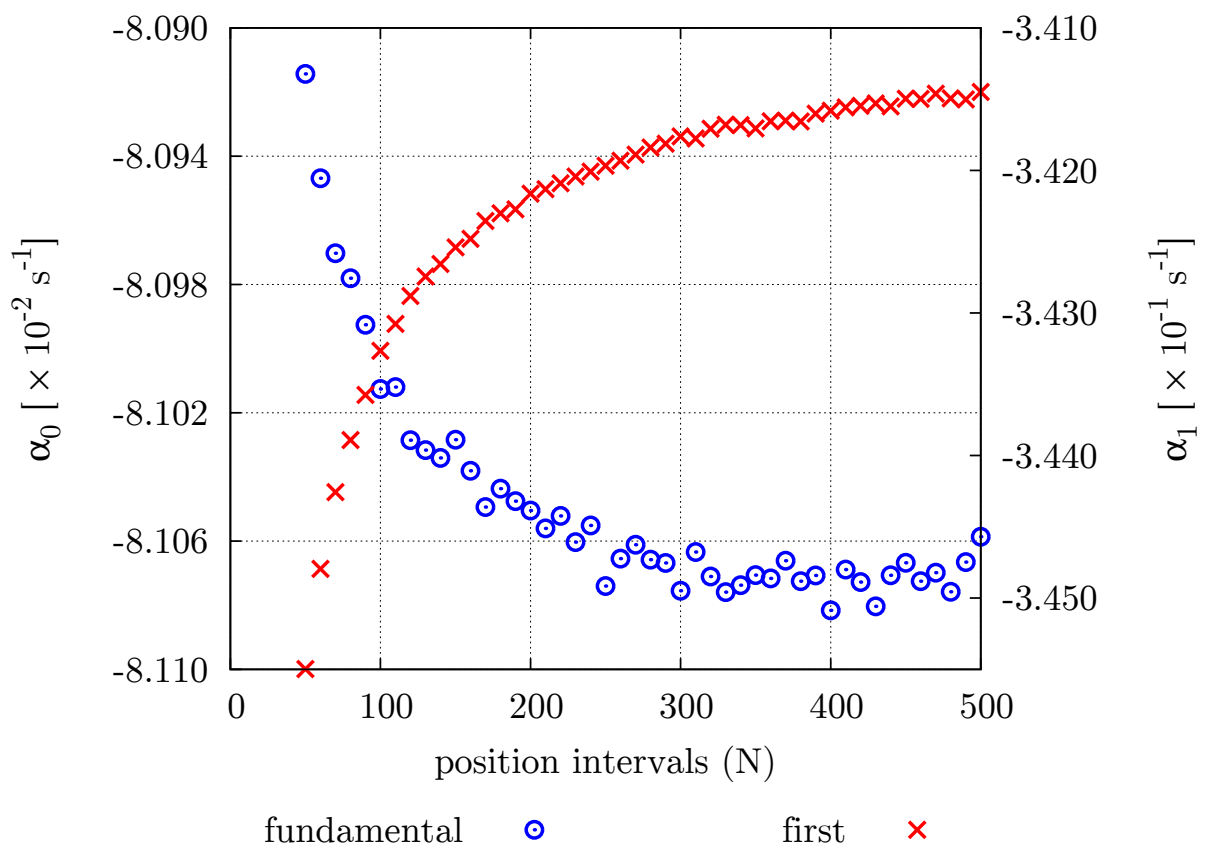

Figure 5.16: The first two TORTE-calculated eigenvalues of the $\Delta=5.0 \mathrm{mfp}$ thick scattering slab approach values as the number of position intervals increases. TORTE uses $M=16$ direction intervals and $200 \times 10^{6}$ active histories for all runs. 


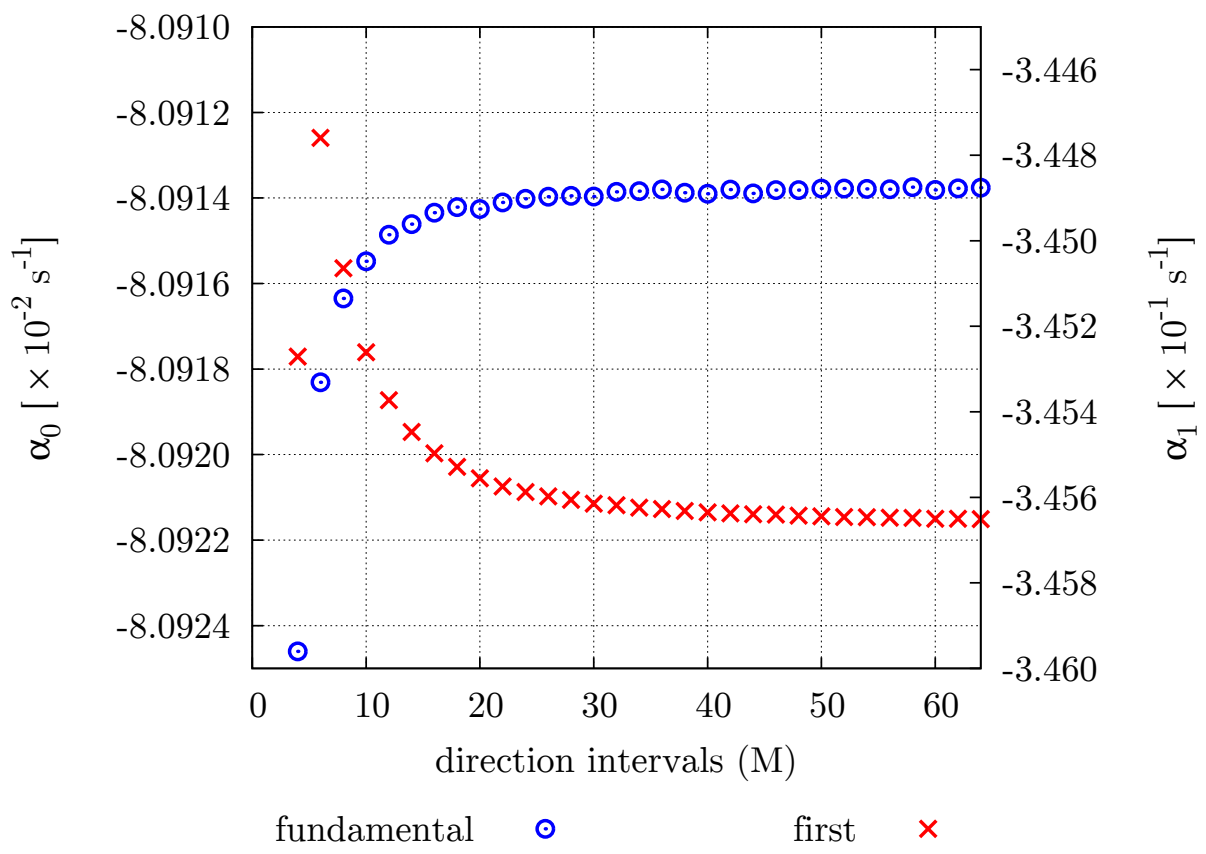

Figure 5.17: The first two TORTE-calculated eigenvalues of the $\Delta=5.0 \mathrm{mfp}$ thick scattering slab converge to values as the number of angular intervals increases. TORTE uses $N=50$ direction intervals and $200 \times 10^{6}$ active histories for all runs, starting with the same random number seed.

There are only three discrete eigenvalues in this slab, and TORTE has more difficulty finding the two higher eigenvalues because of the calculation of the continuum spectra. For larger slabs with more eigenvalues, TORTE does not have this issue and shows tighter agreement with expected eigenvalues. Spectra of heterogeneous onespeed slabs with multiple materials often have similar shapes, but rings of eigenvalues tend to correspond to specific materials. For example, the spectrum of the five-region slab with a central vacuum behaves similarly to the other five-region slabs, except that some rings of eigenvalues extend from the imaginary axis back toward the continuum. These rings of eigenvalues correspond to the central vacuum in the slab: there are still rings in this spectrum that start near the original Corngold limit $\alpha^{*}=-1$.

Increasing the number of position intervals while holding the direction intervals constant is not entirely useful. With an increase in $N$, the thickness of each intervals decreases. With very small intervals, neutrons are more likely to exit in any direction, and are only likely to remain within the interval if they are traveling with very small $\mu$. Thus, a higher direction resolution is desirable. Overall, the eigenvalues trend more with an increase in $N$ compared to an increase in $M$. 


\subsubsection{On the Residual}

The one-speed slab expanded flux solutions show no evidence of a large inseparable part as do the expanded solutions for continuous-energy infinite media. But, the only reason that these expanded flux solutions match TDMC solutions throughout transients is that the eigenfunction expansions include TORTE-calculated eigenvalues that fall within the continuum. Without these additional eigenvalues, the eigenfunction expansions consist of only a few terms, which are unable to describe sudden variations in the flux that is necessary during early times in a solution to a pulsed source. In the interpretation that the only eigenvalues are the discrete ones existing above the Corngold limit, the residual term is large and relatively long-lived. For example, for the multi-region scattering slab there are only four discrete eigenvalues, so the eigenfunction expansion is

$$
\phi(x, t)=\sum_{i=0}^{3} T_{i}(t) \phi_{i}(x)+\zeta(x, t),
$$

where the residual term includes all the higher TORTE-calculated kinetic modes,

$$
\zeta(x, t)=\sum_{i=4}^{N M} T_{i}(t) \phi_{i}(x)+\zeta^{\prime}(x, t)
$$

plus an additional residual term $\zeta^{\prime}(x, t)$ brought about by the smooth expanded flux solution being unable to calculate the abrupt end in the neutron flux at the edges of propagating pulse shapes. If this is the case, the $\zeta(x, t)$ term behaves like those in infinite media: it has large positive and negative parts and decays relatively quickly. For the pulsed transients, this residual term decays by the time neutrons cross the slabs and reach the right boundary.

With a reduction in the overall thickness of the slabs, the fundamental eigenvalue moves closer to the Corngold limit, making it more difficult for the TRMM to resolve this eigenvalue. Calculations with the GFM observe the fundamental eigenvalue retreating into the continuum, in which case there is no expanded flux solution because the neutrons have a very low probability of interacting with a very thin slab. The disappearance of this fundamental eigenvalue into the continuum has not been rigorously proven. As the slabs increase in thickness, more discrete eigenvalues enter the spectrum so that the expanded flux solution is able to describe smooth shapes within the slab. To accurately model the response to pulsed sources, the expanded flux solution always needs the complex portion of the spectrum. In cases where TORTE is 
unable to calculate a discrete eigenvalue close to the Corngold limit, the nearest complex eigenvalues together approximate the contribution to the expanded flux solution that the missing eigenvalue otherwise has.

All expanded flux solutions show the average behavior of the evolution of the flux: it does not show individual realizations resulting from a few particle histories. For example, in the supercritical five-region slab, it is unlikely that neutrons reach the right fuel region because they need to cross the large central absorber. So if a few neutrons start from a source at the left face, it is highly unlikely that they reach the right fuel region and cause a supercritical excursion. But, regardless of the strength of the source used in the expanded flux solution, it still calculates the supercritical result. It is possible to use these expanded solutions to yield probabilities of the occurrence of this supercritical realization.

In the last five-region slab, the expanded flux solution incorrectly oscillates in the central void region but behaves appropriately in the fuel and moderator regions: the eigenfunction expansion does not apply well to large void regions.

\subsection{Continuous-Energy Slabs}

Two problems with geometry and material specifications reflecting thermal reactors (Table 5.9) demonstrate the application of the TRMM combining a continuous-energy phase space with the simplified position-direction phase space of a one-dimensional medium. While the next logical step from one-speed slabs is few-group slabs, there is a lack of benchmark data for multigroup slabs. For all problems, TORTE first divides the energy phase space into $G$ equal-lethargy intervals between $E_{G+1}=0.001 \mathrm{eV}$ and $E_{1}=20 \mathrm{MeV}$. It then sets the floor of the $G$ th energy interval to the minimum energy

Table 5.9: Nuclear data for the continuous-energy slabs.

\begin{tabular}{cccccccc}
\hline \multirow{2}{*}{ Problem } & \multirow{2}{*}{ Material } & \multirow{2}{*}{$N_{d}\left[\mathrm{barn}^{-1} \cdot \mathrm{cm}^{-1}\right]$} & \multicolumn{3}{c}{ Number Ratios } & \multirow{2}{*}{${ }^{235} \mathrm{U}$ [atom \%] } \\
& & & $\mathrm{H}$ & $\mathrm{C}$ & $\mathrm{O}$ & $\mathrm{U}$ & \\
\hline \multirow{3}{*}{ 16A-D } & Fuel 1 & 0.072997 & 4.56 & - & 4.28 & 1 & 1.36 \\
& Fuel 2 & 0.072997 & 4.56 & - & 4.28 & 1 & 1.23 \\
& Fuel 3 & 0.072997 & 4.56 & - & 4.28 & 1 & 1.10 \\
& Reflector & 0.074874 & 2 & - & 1 & 0 & - \\
& Fuel H & 0.078547 & - & 300 & 1.5 & 1 & 2.00 \\
& Fuel M & 0.078547 & - & 300 & 1.5 & 1 & 1.73 \\
& Fuel L & 0.078547 & - & 300 & 1.5 & 1 & 1.46 \\
& Reflector & 0.087341 & - & 1 & 0 & 0 & - \\
\hline
\end{tabular}



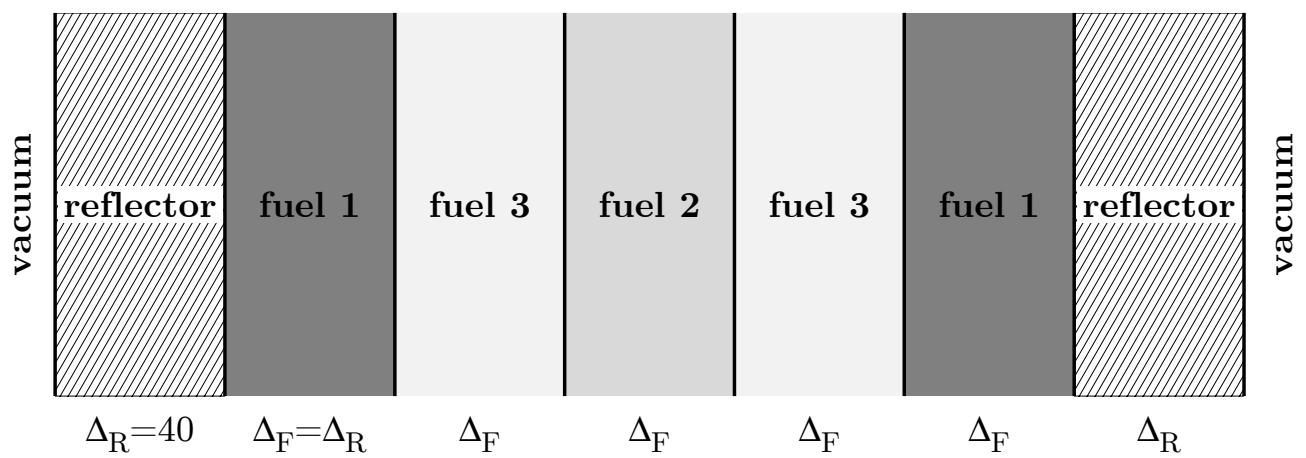

Figure 5.18: The hydrogenous, seven-region slab consists of equal-sized homogeneous subregions of fuel and reflector materials. Measurements are in $\mathrm{cm}$.

$E_{G+1}=E_{\min }=1 \times 10^{-5} \mathrm{eV}$, and sets the floor of the first $(g=1)$ energy interval to $E_{2}=10 \mathrm{MeV}$. There are $J=6$ precursor groups for each slab containing fissile material: there are a total of $J N_{f}$ precursor states. The uranium consists only of ${ }^{238} \mathrm{U}$ and ${ }^{235} \mathrm{U}$, and all continuous-energy cross sections are at room temperature. The rest of the analysis in this chapter uses these two problems.

Problems 16A-D: This is a seven-region slab with five central fuel subregions and two outer reflectors (Figure 5.18). All regions are the same thickness, there are three different fuel enrichments, and the total size of the problem is $\Delta=280.0 \mathrm{~cm}$. The different variations of this problem test the effect of the discretization of the position-energy-direction phase space. TORTE calculates the $k$ eigenvalue of this subcritical slab to be $0.99312 \pm 4 \mathrm{pcm}$.

Problem 17: This is a fifteen-region slab with twelve fuel subregions, and outer and inner reflectors (Figure 5.19). All fuel regions are the same thickness, the re-
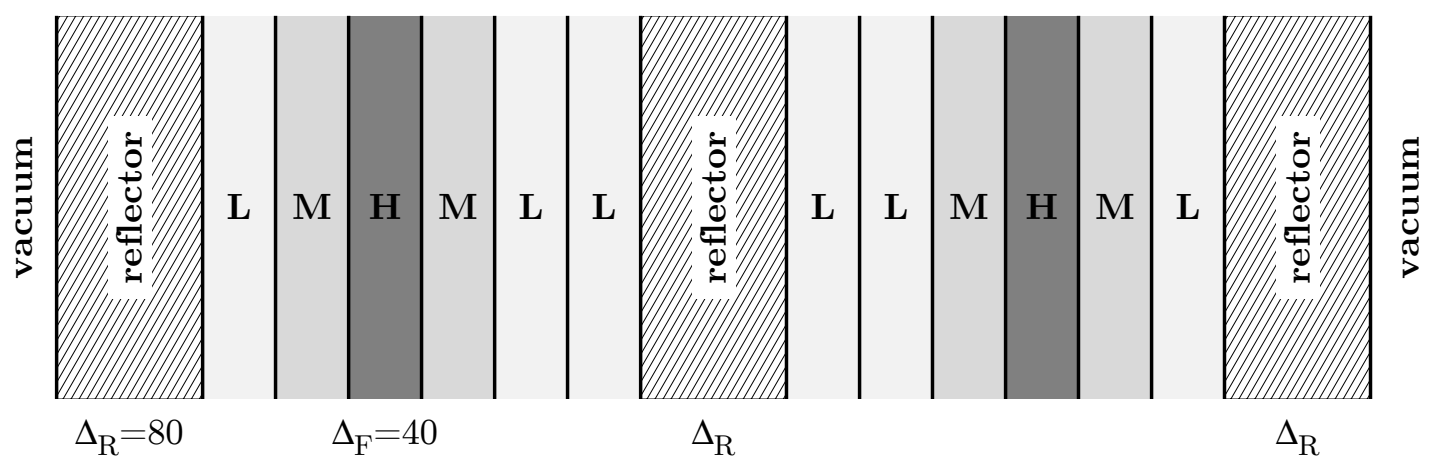

Figure 5.19: The graphite-moderated, fifteen-region slab consists of homogeneous, equalsized fuel subregions and larger outer and inner reflectors. Measurements are in $\mathrm{cm}$. 
flectors are twice as thick as the fuel regions, and the total size of the problem is $\Delta=720.0 \mathrm{~cm}$. The TORTE-calculated $k$ eigenvalue of this slightly supercritical slab is $1.00667 \pm 11 \mathrm{pcm}$.

\subsubsection{Eigenvalue Spectrum}

There is no benchmark data for these continuous-energy slabs, but from theory, the calculated neutron generation times are the order of $10^{-5}$ and $10^{-4}$ seconds for the hydrogenous and graphite-moderated media, respectively. With an effective delayed neutron fraction of $\beta_{\text {eff }}=0.0065$, Eq. (4.25) yields the calculated neutron generations times. These quantities align with theoretical expectations (Table 5.10).

The $\alpha$-eigenvalue spectrum has features similar to those seen for the one-speed slabs, except now these features are repeated throughout the spectrum (Figure 5.20) a number of times correlated to the number of energy intervals TORTE uses. On their own, each section of the spectrum behaves like that of a one-speed slab: there are a few real eigenvalues at the front followed by large circular rings of eigenvalues that for one-speed slabs, fell into the continuum. The largest feature in the spectrum corresponds to the highest energy interval, $10 \mathrm{MeV}<E \leq 20 \mathrm{MeV}$. This feature is so large that the other features in the spectrum are not visible on the same scale. Each successively smaller feature corresponds to the next lowest energy interval. The delayed, prompt fundamental, and first few prompt eigenvalues exist at the very front of the spectrum, $\operatorname{Re}(\alpha)>-1 \times 10^{4} \mathrm{~s}^{-1}$, where all $\alpha$ eigenvalues are all-real. The majority of the eigenvalues fall near the imaginary axis: this is similar to the behavior of continuous-energy infinite-media spectra. Oscillations from kinetic modes corresponding to any of the complex eigenvalues decay quickly.

The reason for the scaling of this spectrum is tied to the large energy ranges over which neutrons interact and the association of the $\alpha$ eigenvalue with neutron speed. At high neutron speeds, events occur rapidly: $\alpha$ eigenvalues with large negative real parts are necessary to describe these transitions. For example, the large real eigenvalues in the feature corresponding to the highest energy interval describe the rapid decay of the fast flux in this thermal medium: with an initial high-energy source,

Table 5.10: Calculated neutron generation times of the two continuous-energy slabs.

\begin{tabular}{clccc}
\hline Problem & Description & $\Lambda[\mu \mathrm{s}]$ & $\rho$ & $\alpha_{p 0}\left[\mathrm{~s}^{-1}\right]$ \\
\hline $16 \mathrm{~A}$ & Hydrogenous & 52.27 & -0.006296 & -244.83 \\
17 & Graphite-Moderated & 135.7 & 0.006671 & 1.2628 \\
\hline
\end{tabular}


the very fast $(E>10 \mathrm{MeV})$ flux decays rapidly in this medium. At thermal neutron speeds, events occur more slowly, and the eigenvalues describing these behaviors fall closer to the imaginary axis.

Due to the discretization of the delayed neutron precursor concentrations over the position phase space, there are $J N_{f}$ delayed eigenvalues and shape eigenfunctions.
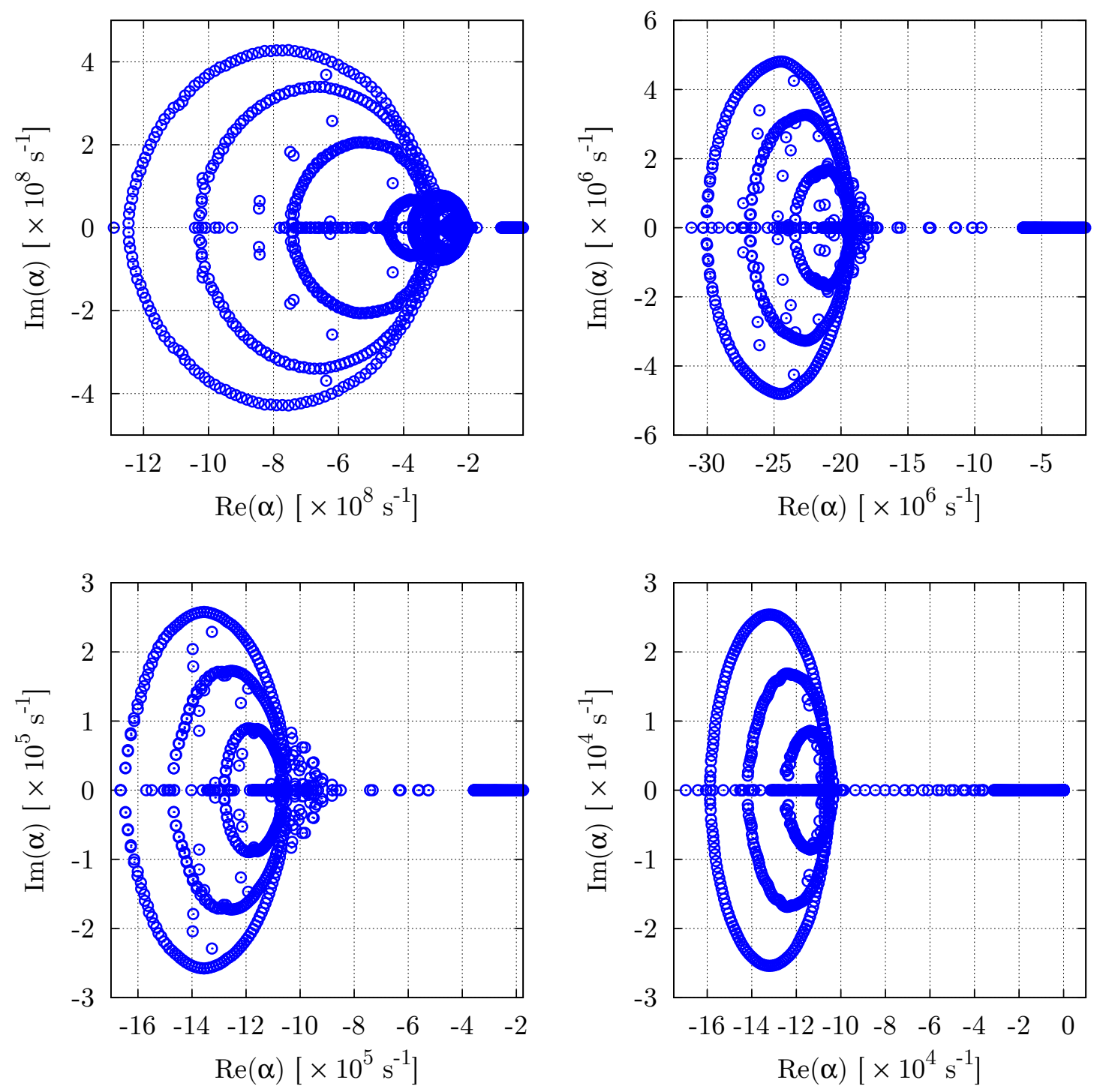

Figure 5.20: The whole $\alpha$-eigenvalue spectrum of the graphite-moderated slab has different features that exist on very different scales. TORTE uses $N=90$ position, $M=8$ direction, and $G=5$ energy intervals, and runs 160 active cycles with $1 \times 10^{6}$ particles per cycle. From top left to bottom right, these features correspond to the five energy intervals: the highest energy interval $g=1, g=2, g=3$, and $g=4$. The short line at the front of the spectrum in the bottom-right plot corresponds to the $g=5$ energy interval. 
The delayed eigenvalues form groups of $N_{f}$ eigenvalues very near a single precursor group decay constant, but with different shape eigenfunctions that peak in one of the $N_{f}$ fissile regions. Summed together, these $N_{f}$ shape eigenfunctions make up a delayed shape eigenfunction $\phi_{d j}$. Because precursor decay occurs on such a different timescale than the prompt neutron effects, it is unnecessary to define the precursor concentrations within the phase space discretization; however, any benefits from having the precursors distributed throughout the position phase space is lost. This includes accounting for mobile fuel or generating constant delayed neutron precursor concentrations distributed in the position phase space.

\subsubsection{Discretization of the Phase Space}

To study the effect that changes to the phase space discretization has on the calculated $\alpha$-eigenvalue spectrum, TORTE runs the subcritical hydrogenous slab several times, in each case, doubling the number of one of the phase space intervals (Table 5.11). In all runs, the calculated prompt fundamental $\alpha$ eigenvalue deviates less than $1.5 \%$ from run A. The eigenvalue trends more negative as TORTE calculates a lower $k$ eigenvalue in the runs with $N=140$ position intervals. A finer energy phase space discretization causes the greatest change in the fundamental eigenvalue, while doubling the direction intervals has little effect. The next few prompt $\alpha$ eigenvalues have similar trends as the fundamental, and the delayed eigenvalues show no trend, other than there being more of them as $N_{f}$ increases.

The whole $\alpha$-eigenvalue spectrum of the hydrogenous slab has five features existing on different scales: there are four features with circular rings of eigenvalues, and a fifth feature near the imaginary axis with just one set of real eigenvalues. As a whole, this

Table 5.11: Phase space discretization and calculated fundamental $\alpha$ eigenvalues of the hydrogenous continuous-energy slabs.

\begin{tabular}{ccccccc}
\hline \multirow{2}{*}{ Run $^{\mathrm{a}, \mathrm{b}}$} & \multirow{2}{*}{$k_{0}^{\mathrm{c}}$} & $\mathrm{N}$ & $\mathrm{G}$ & $\mathrm{M}$ & $\alpha_{p 0}\left[\mathrm{~s}^{-1}\right]$ & \multirow{2}{*}{$\%$ Deviation } \\
\hline $\mathrm{A}$ & 0.99370 & 70 & 5 & 8 & -244.7 & - \\
$\mathrm{B}$ & - & 70 & 5 & 16 & -244.8 & +0.04 \\
$\mathrm{C}$ & - & 70 & 10 & 8 & -240.5 & -1.71 \\
$\mathrm{D}$ & 0.99362 & 140 & 5 & 8 & -247.6 & +1.19 \\
$\mathrm{E}$ & - & 140 & 10 & 8 & -243.3 & -0.55 \\
\hline
\end{tabular}

a TORTE runs 1,000 active cycles with $1 \times 10^{6}$ particles per cycle

b Runs $\mathrm{A} / \mathrm{B} / \mathrm{C}$ and $\mathrm{D} / \mathrm{E}$ use the same random number seeds.

${ }^{c}$ Uncertainties converged to $10 \mathrm{pcm}$. 
undergoes specific changes for each increase in the phase space discretization (Figure 5.21 ). Doubling the position intervals to $N=140$ approximately doubles the radius of the eigenvalue rings in the first four features of the spectrum: the fifth feature just extends further toward the negative region. Doubling the direction intervals to $M=16$ doubles the number of eigenvalue rings in each of the four features, and does not change the set of real eigenvalues near the imaginary axis. Both of these
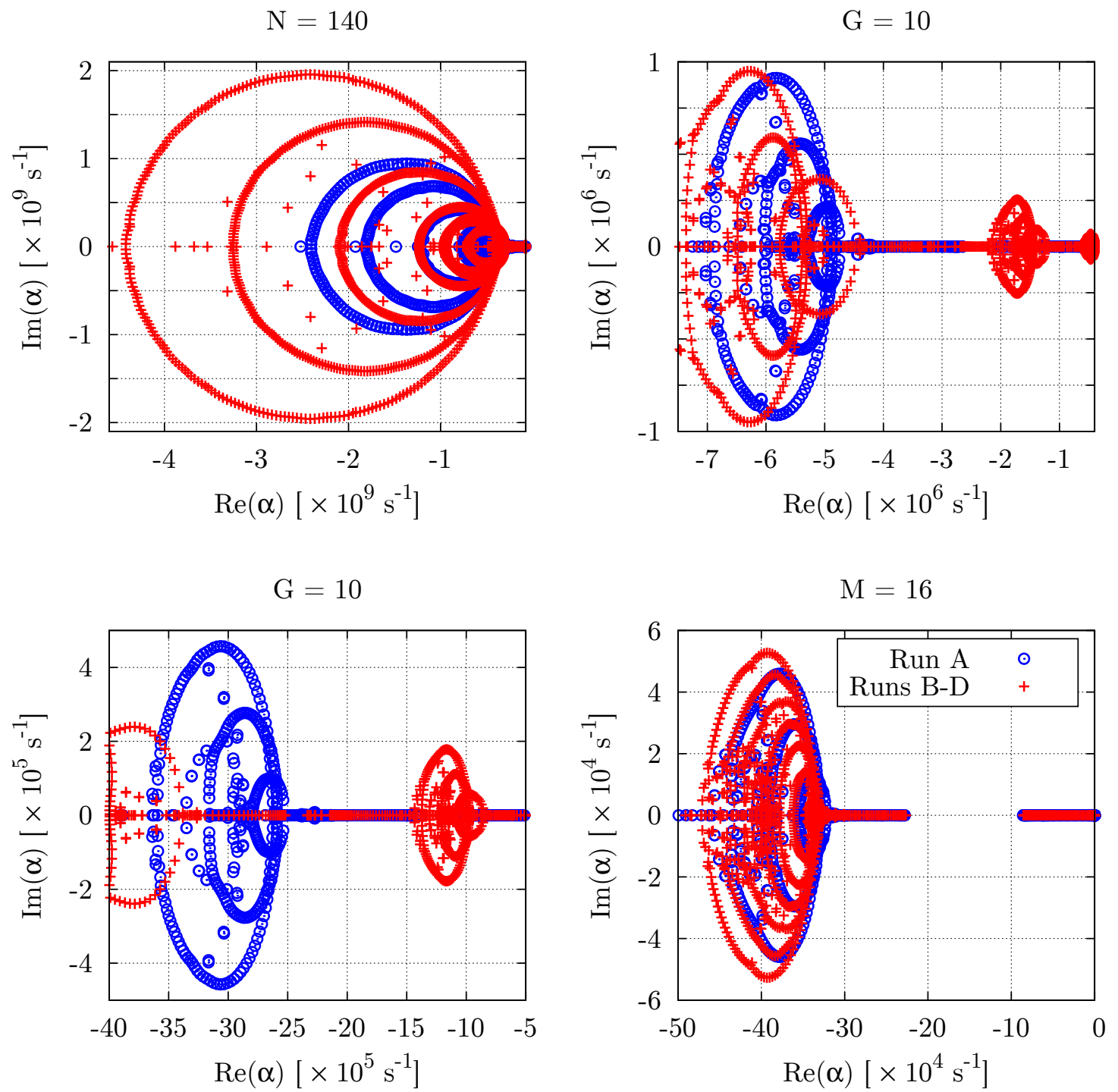

Figure 5.21: Snapshots comparing the spectra of the hydrogenous slab using different phase space discretizations show the effects of a change in the position, energy, or direction intervals on the TORTE-calculated $\alpha$ eigenvalue spectrum. The base-case run A uses $N=70$ position, $M=8$ direction, and $G=5$ energy intervals, and title of each individual snapshot denotes the change to the discretization. 
observations are like those seen for the one-speed slabs. Doubling the energy intervals to $G=10$ increases the number of features in the spectrum, and shifts them due to the different energy intervals. For example, the highest energy interval stays the same in all runs: it corresponds to neutrons in the energy range $E>10 \mathrm{MeV}$. Doubling the energy intervals has little effect on this portion of the spectrum: many eigenvalues remain stationary. But, the energy ranges of the other nine intervals is different, so the neutron speed changes, and with it, the location of the onset of the eigenvalue rings shifts. An increase in the energy intervals has little effect on the set of real eigenvalues towards the imaginary axis.

\subsubsection{Shape Eigenfunctions}

Each shape eigenfunction $\phi_{n}$ corresponding to a calculated $\alpha$ eigenvalue is distributed in the position-energy phase space. For the subcritical hydrogenous slab, the fundamental shape eigenfunction is all-real, all-positive, and peaks in the thermal energy

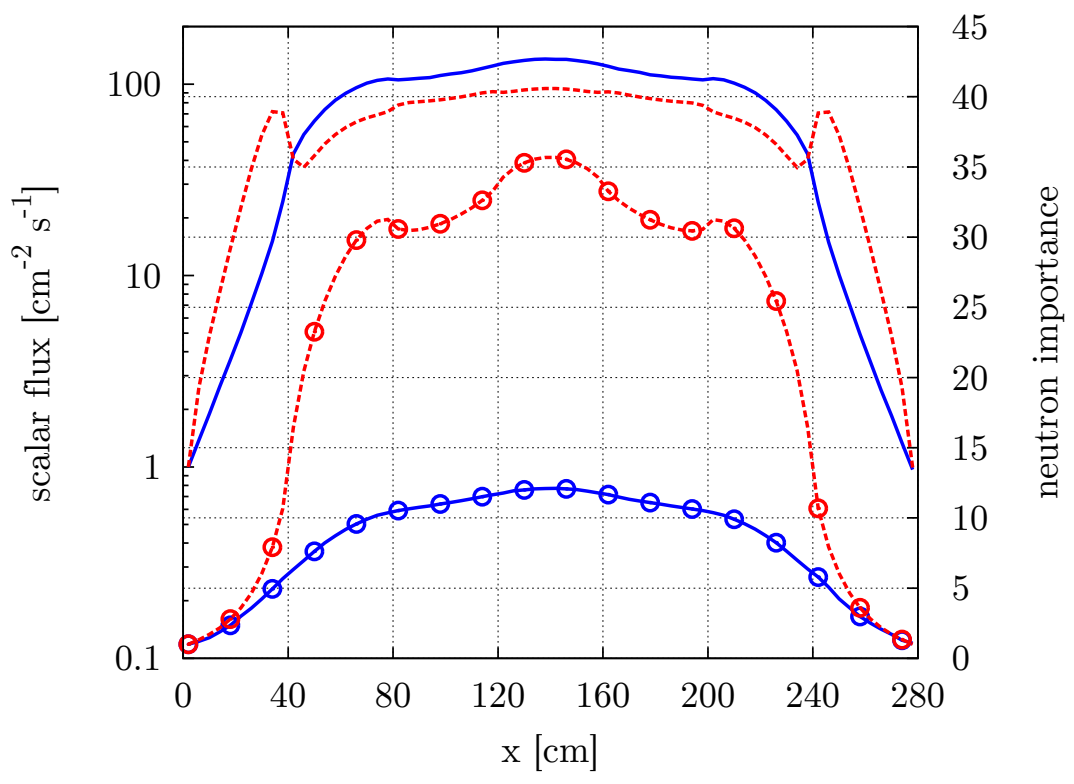

$\begin{array}{rrr}\text { forward (fast) } & \text { adjoint (fast) } & -0- \\ \text { forward (thermal) } & -------\cdot & \text { adjoint (thermal) }\end{array}$

Figure 5.22: The fundamental forward and adjoint shape eigenfunctions of the hydrogenous slab all peak in the center of the fuel. TORTE obtains these eigenfunctions for run A, where the thermal and fast fluxes correspond to energy intervals $g=5$ and $g=1$, respectively. These are normalized for each energy interval: the fast forward and adjoint shape eigenfunctions are actually three orders of magnitude smaller than the fundamental. 
interval $g=5$. Distributed in the position phase space, the shape function corresponding to each energy interval is symmetric and behaves differently depending on the energy of the neutrons (Figure 5.22). The thermal flux has peaks in the reflectors adjacent to the fuel regions, and has a central peak at the middle of the fuel regions. The fast flux peaks towards the center of the fuel regions as well, but has no flux peaks in the reflector regions. The most important thermal neutrons to the asymptotic solution are located at the center of the core: these neutrons are unlikely to escape the fuel regions without fissioning, and thus will contribute significantly to expected detector response. The importance of fast neutrons varies less over the slab. Note that the unnormalized fast flux is three orders of magnitude smaller than the thermal flux: in the asymptotic solution, relatively few neutrons exist in the very high energy range.

In the continuous-energy infinite media, the delayed and prompt fundamental eigenfunctions for thermal media were distributed similarly in the energy phase space. For the subcritical hydrogenous slab, the effect of kinetic distortion is apparent in the shape eigenfunctions distributed in the position phase space (Figure 5.23). The

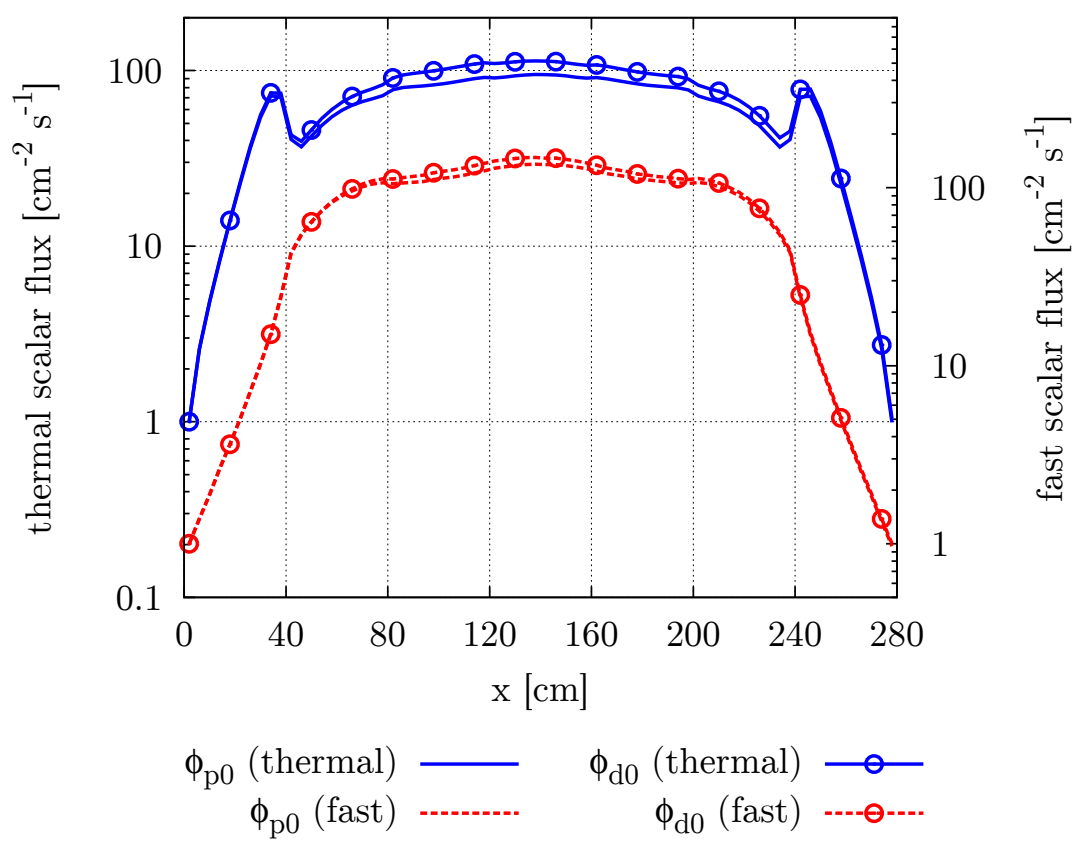

Figure 5.23: A comparison of the prompt fundamental and delayed fundamental shape eigenfunctions shows the small effect of kinetic distortion in the hydrogenous slab. These are the shape eigenfunctions of run A, where the thermal and fast fluxes correspond to the energy intervals $g=5$ and $g=1$, respectively. The delayed shape eigenfunction is the sum of the eigenfunctions corresponding to a group of $N_{f}$ similar eigenvalues. 
prompt fundamental and delayed shape eigenfunctions agree in the reflector regions, but the delayed shape eigenfunctions are slightly more peaked in the fuel regions, where this increase is more pronounced in the thermal than in the fast shape eigenfunction. Physically, the delayed kinetic modes dominate the expanded flux solution after the prompt fundamental kinetic mode decays: the mechanism that drives the delayed modes is the decay of neutron precursors. Precursors are only located in regions containing fuel, so the delayed shape eigenfunctions are greater in those regions. This increase is greater in the thermal flux due to the softer spectrum of delayed neutron emission.

The behavior of higher shape eigenfunctions progresses as expected from theory. For the thermal energy interval of the graphite-moderated slab, successively higher, all-real eigenfunctions have negative and positive parts (Figure 5.24) and cross the $x$-axis and increasing number of times. The fundamental thermal shape eigenfunction peaks in the central reflector, and has peaks in the outer reflectors adjacent to the fuel.

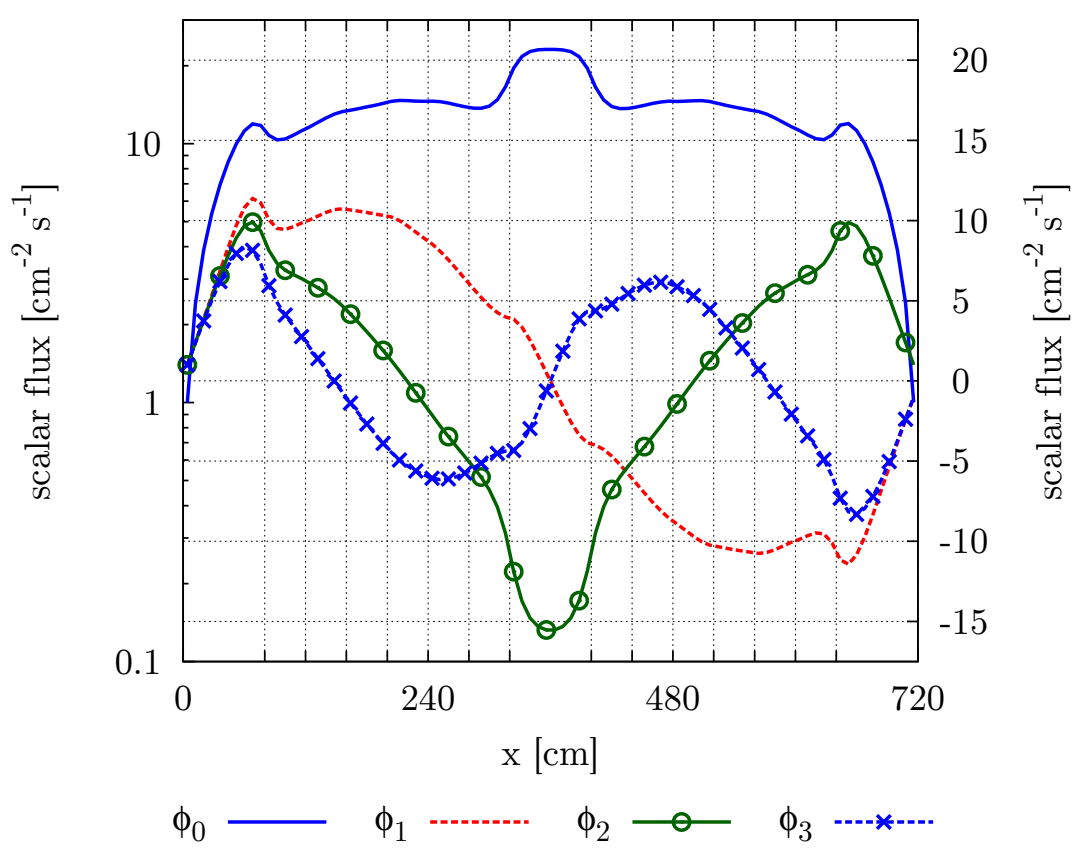

Figure 5.24: The first four thermal shape eigenfunctions $\phi_{n}$ of the graphite-moderated slab cross the $x$-axis $n$ times. These eigenfunctions correspond to the last energy interval, $g=2$. 


\subsubsection{Flux Transients}

Application of the eigenfunction expansion to these continuous-energy slabs delivers solutions distributed in the position-energy phase space. Analysis of these expanded flux solutions shows the effects of individual calculated $\alpha$ eigenvalues in the spectrum.
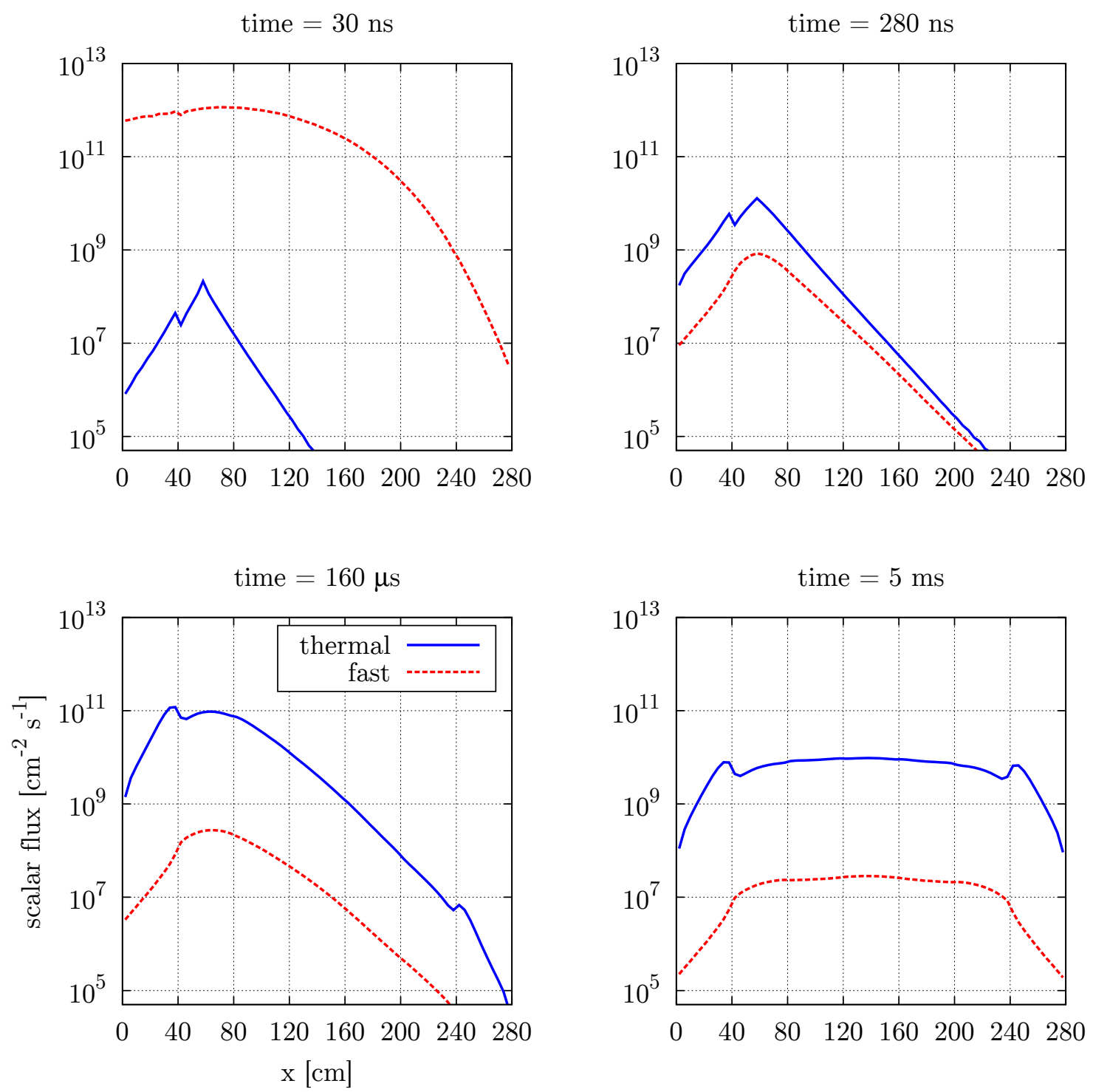

Figure 5.25: Snapshots of the expanded flux solution show the propagation of the initial high-energy pulse and the increase in the thermal flux as these high-energy neutrons slow down in the hydrogenous slab. TORTE uses the run A phase space discretization, and defines the initial source as a pulse at $t=0$ with strength $Q_{0}=1 \times 10^{15} \mathrm{~cm}^{-3} \cdot \mathrm{s}^{-1}$ and no initial precursor concentrations. The source is in the highest energy interval $g=1$ in the $n=15$ slab distributed equally in all $M$ directions. This source design approximates a $E=14.1 \mathrm{MeV}$ pulse in the left-most fuel region at $x=58.0 \mathrm{~cm}$. The fast and thermal fluxes correspond to energy intervals $g=5$ and $g=1$, respectively. 
The expanded flux solution for the hydrogenous slab reacting to a high-energy pulse in the left-most fuel region begins sharply peaked in the highest energy interval (Figure 5.25). At $t=30 \mathrm{~ns}$, high-energy neutrons from the initial pulse reach the right end of the slab. The thermal flux is peaked in the position interval of the initial source, but is several orders of magnitude smaller than the fast flux because neutrons are still slowing down to thermal energies. At $t=280 \mathrm{~ns}$, the fast flux decreases rapidly as high-energy neutrons exit the slab and slow down to thermal energies. The thermal flux overtakes the fast flux in magnitude. At $t=160 \mu \mathrm{s}$, the thermal flux takes on a smooth asymmetric shape as significant numbers of thermal neutrons reach the right end of the slab. The fast flux mimics the asymmetric shape of the thermal flux, without the flux peaks in the reflectors. At $t=5 \mathrm{~ms}$, the expanded flux solution approaches the symmetric prompt fundamental kinetic mode. The solution shows the difference in magnitude between the fast and thermal fluxes. After this time, the expanded flux solutions decrease at a rate dictated by the prompt fundamental eigenvalue, until the prompt kinetic mode decays away and delayed modes dominate the flux solution at approximately $t=60 \mathrm{~ms}$.

The difference in the behavior of the fast and thermal fluxes between $t=30$ and $t=280$ ns shows the reason for the different sets of features in the $\alpha$-eigenvalue spectrum. Over this time, the shape and magnitude of the fast flux changes significantly, while the thermal flux shape remains the same and only increases. This represents the decay of the highest kinetic modes corresponding the the $\alpha$ eigenvalues at the very extent of the spectrum. By $t=280 \mathrm{~ns}$, the kinetic modes of these eigenvalues contribute insignificantly to the expanded flux solution, and the shape eigenfunctions of lower eigenvalues dictate the shape of the fast flux.

An alternate visualization of the expanded flux solution is the response of an ideal detector, like a fission chamber, placed in the right fuel region (Figure 5.26). This detector responds mostly to thermal neutrons (Table 5.12). Plotted on a linear time scale, the detector response shows the three characteristic time intervals sought during a pulsed-neutron experiment. This also shows the short time frame, approximately $t=15 \mathrm{~ms}$, from which measurements possibly yield a fit for the fundamental eigenvalue. Spatially, the detector responses from different position intervals do not vary as much as they do in a three-dimensional model, because only one-dimensional kinetic modes are present. Plotted on a logarithmic time scale, the detector response shows the reaction to the high-energy pulse due to the small probability of detecting high-energy neutrons. 


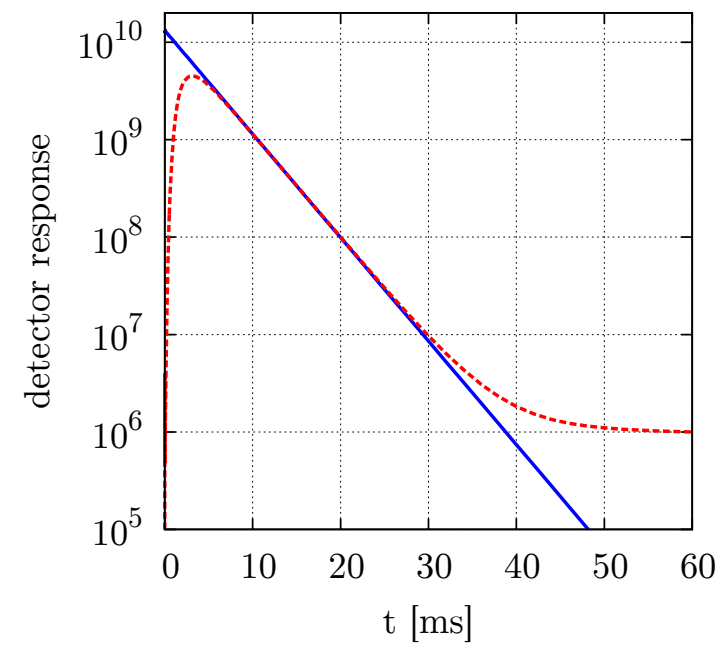

prompt fundamental

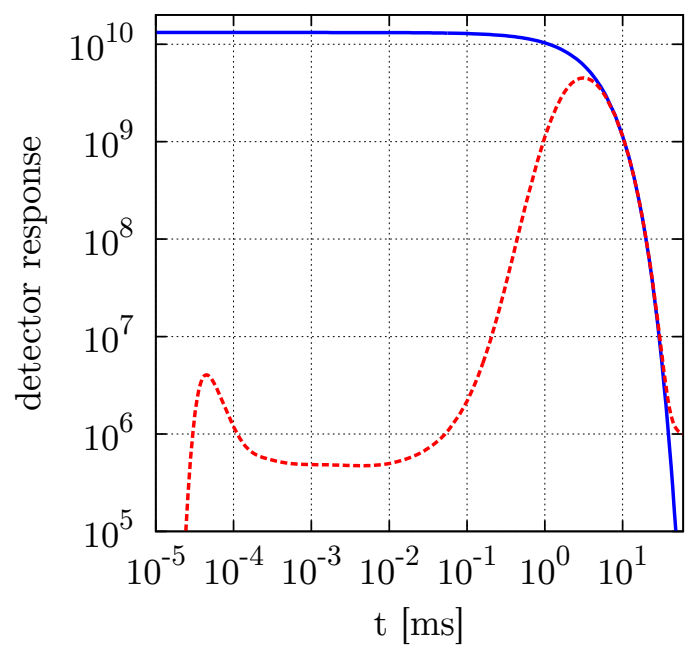

all modes

Figure 5.26: Responses of an ideal detector in the right-most fuel region shows a typical detector response obtained during a pulsed-neutron experiment (left) and one scaled to show the short-time effect of the high-energy pulse (right). These are combinations of the flux in slab $n=60$ and a detector cross section. Also shown is the detector response obtained with the expansion with only the prompt fundamental mode. The selection of this slab approximates a $4 \mathrm{~cm}$ thick detector centered at $x=238.0 \mathrm{~cm}$.

\subsection{Discussion}

The research Monte Carlo code TORTE-1D implements the TRMM for onedimensional media to demonstrate its ability to accurately calculate $\alpha$ eigenvalues and use the entire spectrum to obtain expanded flux solutions. For the five one-speed scattering, multiplying, and mixed material slabs in the GFM benchmark, TORTE$1 \mathrm{D}$ is able to reliably calculate the $k$ eigenvalue and $\alpha$-eigenvalue spectrum. These calculated eigenvalues match GFM results for the real discrete portion of the eigenvalue spectrum, with TORTE-1D calculating excess eigenvalues within the continuum. As the number of position and direction intervals increases, TORTE-1D yields better estimates of the discrete eigenvalues, although the trend in the improvement is small over the observed number of intervals. Increasing the number of position intervals has a much larger effect than direction intervals: the calculated eigenvalues trend

Table 5.12: Simulated cross sections for obtaining the ideal detector response of the hydrogenous slab.

\begin{tabular}{cccccc}
\hline$g$ & 1 & 2 & 3 & 4 & 5 \\
\hline$\Sigma_{d g}$ & 0.0005 & 0.0040 & 0.0069 & 0.0961 & 0.8925 \\
\hline
\end{tabular}


very little after $M=8$ direction intervals. The selection of $N$ and $M$ determines the extent and size of the $\alpha$ eigenvalues calculated within the continuum. Calculated shape eigenfunctions match those provided by the GFM. For these one-speed slabs, the expanded flux solutions match TDMC solutions because of the excess eigenvalues calculated within the continuum. This effectively reduces the computed residual.

For continuous-energy, hydrogenous and graphite-moderated slabs, TORTE-1D calculates a fundamental $\alpha$ eigenvalue in line with theoretical expectations. The $\alpha$ eigenvalue spectrum behaves like those in the one-speed slabs, but with multiple features for the different energy intervals. In the phase space discretization, the number of direction intervals is again observed to have the least effect on the calculated fundamental eigenvalue, while the number of energy intervals has the largest. Still, the trend in this fundamental eigenvalue is small over the runs observed. Calculated shape eigenfunctions are as expected, while the calculated delayed eigenfunctions show the effect of spatial kinetic distortion in the fuel regions. The application of the eigenfunction expansion to describe the reaction of the hydrogenous slab to a pulse source shows the ability of the TRMM to calculate the time-dependence of the neutron flux in the slab. The behavior of the flux in different energy intervals shows the reason behind the multiple features in the $\alpha$-eigenvalue spectrum. The combination of an ideal detector cross section and the neutron flux gives the ideal response at a given position in the slab: this is useful for the analysis of pulsed-neutron experiments.

The expanded flux solutions show the difference between the actual solution and that predicted by only the fundamental mode. Before the higher modes decay, the fundamental mode is an incorrect solution for the time dependent flux. The eigenfunction expansion has difficulties predicting behavior from sources that demand finer angular intervals than are used for tallying the TRM, such as a mono-directional source. Also, desired sources in empty states is a similar issue. As a result of the direction phase space discretization, purely-absorbing media present some problems for the eigenfunction expansion for the shortest times. Also, sharp changes in the neutron flux, such as the front edge of a source pulse, are modeled by a very steep decrease in neutron flux in expanded flux solutions. The TRMM does not apply well to slabs with very large void regions.

\section{References}

[1] FORTRAN90. American National Standards Institute, 1991. 
[2] L. L. Carter and E. D. Cashwell, "Particle-Transport Simulation with the Monte Carlo Method," Tech. Rep. TID-26607, Los Alamos National Laboratory, 1975.

[3] E. Anderson, Z. Bai, C. Bischof, S. Balackford, J. Demmel, J. Dongarra, J. Du Croz, A. Greenbaum, S. Hammarling, A. McKenney, and D. Sorensen, LAPACK Users' Guide. Philadelphia, Pennsylvania: Society for Industrial and Applied Mathematics, Third ed., 1999.

[4] D. E. Kornreich and D. K. Parsons, "Time-eigenvalue Calculations in Multi-region Cartesian Geometry Using Green's Functions," Annals of Nuclear Energy, vol. 32, pp. 964-985, 2005.

[5] R. S. Modak and A. Gupta, "A Simple Scheme for the Direct Evaluation of the Time-Eigenvalues of Neutron Transport Equation," Annals of Nuclear Energy, vol. 30, pp. 211-222, 2003. 


\section{CHAPTER 6}

\section{Three-Dimensional Eigenvalues and Eigenfunctions}

This chapter discusses the implementation of the TRMM to three-dimensional media, where the TRM characterizes neutrons moving through a realistic position-directionenergy phase space. Because considerable work is necessary when including multiple dimensions in a Monte Carlo code, the TRMM is integrated into the open-source Monte Carlo code OpenMC [1]. Benefits of this code are availability, small size, simplicity, and ease of use, though integration into a large production code, e.g., MCNP5/6 [2], is similar. This requires some minor edits to OpenMC because it does not handle time-dependent problems. Problems consist of realistic geometries with vacuum boundary conditions: in this implementation, the TRMM does not handle reflective or periodic boundary conditions.

Three problems demonstrate the capabilities of the TRMM as applied to threedimensional media. The first problem is a homogeneous, multiplying cube to study some effects of discretizing the large position phase space, show the reliability of the TRMM in calculating $\alpha$ eigenvalues, demonstrate convergence of eigenvalues and eigenfunctions, and calculate the expanded flux solutions. Next is the application to the small fast-burst reactor CALIBAN, demonstrating the ability to calculate higher shape eigenfunctions and the behavior of the fundamental eigenvalue as the reactivity increases from a slightly critical state to a prompt supercritical state. Finally, the TRMM application to the FSV reactor demonstrates the calculation of some of the dominant kinetic modes of the reactor as well as the fundamental prompt $\alpha$ eigenvalue. In the first two problems, ratios of calculated $\alpha$ eigenvalues are compared against predictions from diffusion theory. In the latter two problems, some measured data provides a gauge of the accuracy of the TRMM in determining the $\alpha$ eigenvalues. 


\subsection{Code (OpenTRMM)}

The physics engine in OpenMC replaces the simplified physics treatments in TORTE and TORTE-1D, and only the functions necessary to build the TRM carry over: transition rate tallies, matrix building routines, eigenvalue solvers, and post-processing routines. Additionally, edits to portions of the OpenMC code are necessary to make TRM tallies during the neutron random walk, and to sample a decay time from a delayed neutron precursor group. This new code consists of a patch file and three modules with approximately 20 subroutines. The patch file is minimally intrusive, adding approximately 50 new lines to the OpenMC code, with most of the work being completed outside in the module subroutines. The TRM tallies add some computational time because they perform operations after each sampled collision or leakage event. Also, the current state of the neutron must be constantly identified. While OpenMC does not handle time-dependent problems, the TRMM is able to extract time-dependent information during the random walk. The combined code is called OpenTRMM.

For infinite and one-dimensional media, LAPACK directly determines all eigenvalues of the forward and adjoint matrices by reducing the matrix to Schur form. This is possible because the size of the state space is reasonable. With a three-dimensional phase space, the TRM is increasingly large and difficult to work with due to memory limitations. Furthermore, LAPACK neither handles sparse matrix structures nor performs sparse matrix routines. Thus, OpenTRMM uses LAPACK direct solvers only for problems with a smaller defined phase space discretization.

Due to these concerns, OpenTRMM adopts a sparse matrix structure and implements an iterative eigenvalue solver, the Implicitly-Restarted Arnoldi Method (IRAM), through the linear algebra package ARPACK [3]. This method reliably determines a few $\alpha$ eigenvalues in the spectrum without destroying the sparse structure of the TRM as does a direct method. OpenTRMM stores the TRM in a Compressed Row Storage (CRS) format, storing a row pointer, column indices, and values of only those elements that are non-zero. An additional benefit is that transposing the TRM is as simple as changing the storage format to Compressed Column Storage (CCS): no values are moved within memory. These storage formats are $100 \%$ compressed.

Because of the way IRAM determines eigenvalues, it has difficulties distinguishing eigenvalues in clusters [4]. As shown in the previous two chapters, the $\alpha$ eigenvalue spectrum has many features, including sets of clustered eigenvalues: this is especially true for the $J N_{f}$ delayed eigenvalues brought about by discretizing the 
precursor concentrations over the position phase space. Thus, for the implementation of IRAM, OpenTRMM deletes these delayed states, and subsequent calculations yield only prompt $\alpha$ eigenvalues and their shape eigenfunctions. OpenTRMM handles empty states in the TRM the same way.

\subsection{The Hydrogenous Cube}

Problem 18: This is a large, hydrogenous cube with a $H=200 \mathrm{~cm}$ side length. It consists of a homogeneous mixture of water and natural LEU, with some boron poison (Table 6.1). In the position phase space discretization, each position interval runs the whole axial length of the problem: all results of this three-dimensional problem reduce to two dimensions. This is a subcritical configuration.

Table 6.1: Nuclear data for the hydrogenous cube.

\begin{tabular}{ccccc}
\hline$N_{d}\left[\mathrm{barn}^{-1} \cdot \mathrm{cm}^{-1}\right]$ & \multicolumn{4}{c}{ Number Ratios } \\
& $\mathrm{H}_{2} \mathrm{O}$ & $\mathrm{UO}_{2}$ & $\mathrm{~B}[\mathrm{ppm}]$ & ${ }^{235} \mathrm{U}[\%]$ \\
\hline 0.073212 & 3 & 1 & $1300^{\mathrm{a}}$ & 2.619 \\
\hline
\end{tabular}

${ }^{a}$ Natural boron concentration in water.

\subsubsection{Eigenvalue Spectrum}

Three separate runs demonstrate the relative indifference in the calculation of the prompt fundamental $\alpha$ eigenvalue to the position phase space discretization (Table 6.2). In run $B$, the position interval mesh increases from $15 \times 15$ to $25 \times 25$. Runs $B$ and $\mathrm{C}$ use the same phase space discretization, but run $\mathrm{C}$ uses more particle histories. The higher eigenvalues change significantly as the position space discretization increases, suggesting that the $15 \times 15$ spatial mesh is insufficient for calculations of higher modes of this problem. Computationally, the phase space discretizations in Runs B and C generate a TRM of size $18,750 \times 18,750$. This represents nearly the maximum-sized matrix whose eigenvalues are reasonably obtained via direct methods: solving for all 18,750 eigenvalues for these runs takes a little less than a day with LAPACK running on a single core of an Intel Core i7 CPU, and requires $25 \mathrm{~GB}$ of memory.

The majority of the entire $\alpha$-eigenvalue spectrum is all-real, where eigenvalues group on different scales due to the energy phase space discretization, as seen in the one-dimensional continuous-energy spectra. Replacing the distinct rings of eigenvalues are patches of clustered complex eigenvalues with small imaginary parts: the ratio between the imaginary and real parts of the eigenvalues is always less than unity. 
Table 6.2: Position phase space discretization and calculated prompt fundamental $\alpha$ eigenvalues of the hydrogenous cube runs.

\begin{tabular}{ccccccc}
\hline \multirow{2}{*}{ Run $^{\mathrm{a}, \mathrm{b}, \mathrm{c}}$} & \multirow{2}{*}{$k_{0}$} & $\mathrm{Intervals}$ & \multirow{2}{*}{$\alpha_{p 0}\left[\mathrm{~s}^{-1}\right]$} & \multirow{2}{*}{$\%$ Deviation } \\
& & $\mathrm{N}$ & $\mathrm{G}$ & $\mathrm{M}$ & & \\
\hline $\mathrm{A}$ & $0.99693 \pm 9 \mathrm{pcm}$ & 225 & 3 & 8 & -454.3 & - \\
$\mathrm{B}$ & $0.99708 \pm 6 \mathrm{pcm}$ & 625 & 3 & 8 & -448.3 & -1.21 \\
$\mathrm{C}$ & $0.99704 \pm 2 \mathrm{pcm}$ & 625 & 3 & 8 & -451.6 & -0.62 \\
\hline
\end{tabular}

\footnotetext{
${ }^{a}$ Run A uses $190 \times 10^{6}$ active histories.

b Run B uses $480 \times 10^{6}$ active histories.

${ }^{\text {c }}$ Run $\mathrm{C}$ uses $5 \times 10^{9}$ active histories.
}

These clusters are irregular in shape and may contain circular voids containing no eigenvalues.

To test the reliability of the IRAM in calculating the proper desired $\alpha$ eigenvalues, ARPACK determines the 25 smallest-magnitude eigenvalues of the same TRM. After deleting the delayed neutron states to reduce the work of IRAM, this yields the fundamental and next few prompt eigenvalues, as calculated by direct methods. These eigenvalues match to machine precision.

While no calculated or measured data for this hydrogenous cube exists, one-speed diffusion theory yields a back-of-the-envelope calculation of the ratios of the first few expected $\alpha$ eigenvalues [5]. From diffusion theory, the first few prompt eigenvalues follow as

$$
\alpha_{p}=\frac{k_{\infty}-\left(1+L^{2} B_{p}^{2}\right)}{l_{\infty}}
$$

where $L$ is the diffusion length, $k_{\infty}$ is the infinite-medium multiplication factor, $l_{\infty}$ is the infinite-medium neutron generation time, and

$$
B_{p}^{2}=\left(\frac{i_{x} \pi}{\tilde{H}}\right)^{2}+\left(\frac{i_{y} \pi}{\tilde{H}}\right)^{2}+\left(\frac{i_{z} \pi}{\tilde{H}}\right)^{2}
$$

where $\tilde{H}$ is some extrapolated cube side length and $\left(i_{x}, i_{y}, i_{z}\right)$ is the triplet of integers identifying the eigenvalue. While the diffusion length and infinite-medium generation times are unknown, it is possible to approximate the ratios of the first few eigenvalues without these quantities (Table 6.3), assuming that $k_{\infty}=1.03$, the delayed neutron fraction $\beta=0.0065$, and a zero extrapolation distance $\tilde{H}=H$. While these ratios are not exact matches to those calculated by the TRMM, the loose agreement is an indication that OpenTRMM is calculating $\alpha$ eigenvalues of this system. Note that the z-direction triplet $i_{z}$ is unity for the calculation of all ratios: this reflects the 
Table 6.3: The first few calculated prompt $\alpha$ eigenvalues of the hydrogenous cube.

\begin{tabular}{ccrrr}
\hline & \multirow{3}{*}{$\begin{array}{c}\text { OpenTRMM } \\
\left(_{x}, i_{y}, i_{z}\right)\end{array}$} & $\alpha_{p n}\left[\mathrm{~s}^{-1}\right]$ & $\alpha_{p n}: \alpha_{p 0}$ & \multicolumn{1}{c}{$\alpha_{p 0}$} \\
\hline 1 & $(1,2,1)$ & -2788 & 6.17 & 5.12 \\
2 & $(2,1,1)$ & -2788 & 6.17 & 5.12 \\
3 & $(2,2,1)$ & -4969 & 11.00 & 9.23 \\
4 & $(1,3,1)$ & -6627 & 14.67 & 11.98 \\
5 & $(3,1,1)$ & -6630 & 14.68 & 11.98 \\
6 & $(2,3,1)$ & -8568 & 18.97 & 16.10 \\
7 & $(3,2,1)$ & -8571 & 18.98 & 16.10 \\
8 & $(1,4,1)$ & -11440 & 25.32 & 21.59 \\
9 & $(4,1,1)$ & -11440 & 25.32 & 21.59 \\
10 & $(3,3,1)$ & -11810 & 26.15 & 22.97 \\
\hline
\end{tabular}

${ }^{\mathrm{a}}$ Ratios from one-speed diffusion theory.

choice of a single axial position interval, and OpenTRMM calculates dual-multiplicity eigenvalues instead of those with triple-multiplicity. This is different than assuming that there is no axial dimension, in which case $B_{p}^{2}$ contains only two terms and the ratios of higher eigenvalues is much smaller.

\subsubsection{Shape Eigenfunctions}

Along with the ratios of the first few prompt eigenvalues, diffusion theory predicts the general progression of the prompt shape eigenfunctions of this homogeneous medium, which follow as

$$
\phi(x, y, z)=f\left(\frac{i_{x} \pi x}{\tilde{H}}\right) f\left(\frac{i_{y} \pi y}{\tilde{H}}\right) f\left(\frac{i_{z} \pi z}{\tilde{H}}\right)
$$

where

$$
\begin{aligned}
& f(u)=\cos (u) \quad \text { for odd } \quad i_{x}, i_{y}, i_{z} \\
& f(u)=\sin (u) \quad \text { for even } \quad i_{x}, i_{y}, i_{z}
\end{aligned}
$$

The first few shape eigenfunctions from the TRMM are expected to agree to diffusion theory (Figure 6.1). The first shape eigenfunction corresponds to the prompt fundamental $\alpha$ eigenvalue and the triplet $(1,1,1)$, which is an all-positive, smooth cosine shape in all three dimensions (Figure 6.2). The next three shape eigenfunctions correspond to the same eigenvalue and the triplets $(1,1,2),(1,2,1)$, and $(2,1,1)$ : these have the same shape, with a rotation to form a sine shape in one of the three directions. But, with the single axial position interval, the triplet corresponding to $(1,1,2)$ does not exist to the TRMM, and the spectrum has only two repeated eigenvalues. 
Mode $(1,1,1)$

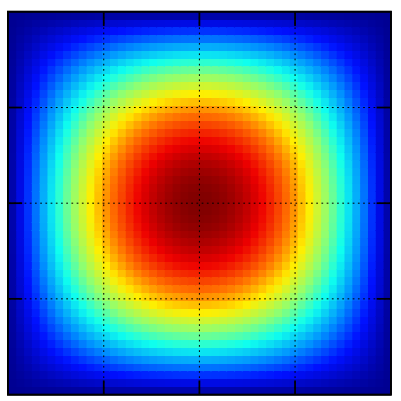

Mode $(2,2,1)$

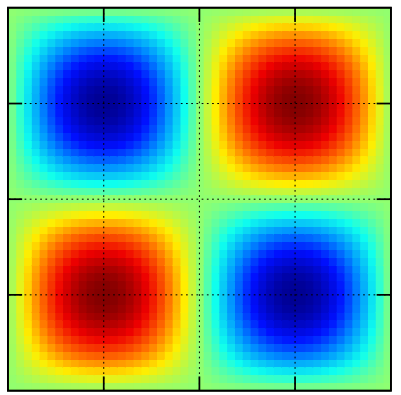

Mode $(1,2,1)$

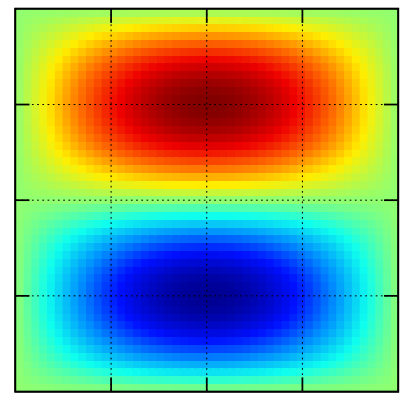

Mode $(3,2,1)$

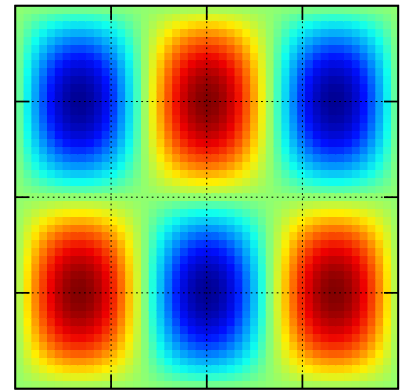

Mode $(2,1,1)$

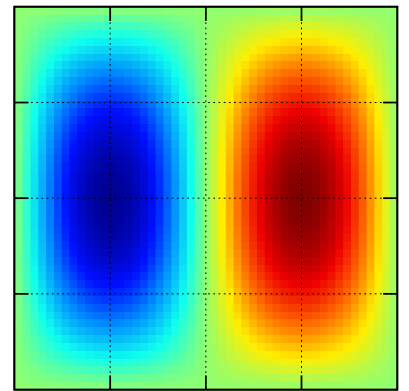

Mode $(3,3,1)$

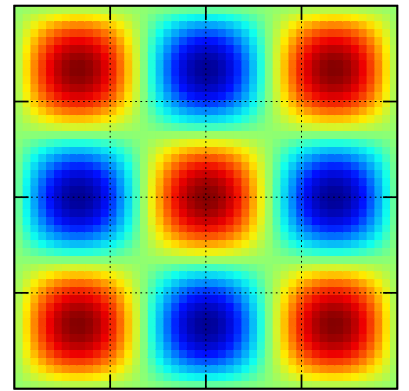

Figure 6.1: Diffusion theory predicts the two-dimensional, prompt fundamental shape eigenfunctions of the hydrogenous cube. Axially, these eigenfunction have an assumed cosine shape. The $x$ and $y$ dimensions are the same as shown for the fundamental mode.

Thus, the shape eigenfunctions of all higher eigenvalues with multiplicity follow as

$$
\phi(x, y, z)=f\left(\frac{i_{x} \pi x}{\tilde{H}}\right) f\left(\frac{i_{y} \pi y}{\tilde{H}}\right) \cos \left(\frac{\pi z}{\tilde{H}}\right)
$$

which is different than simply negating the axial dependence of the shape eigenfunction. The line formed by each higher shape eigenfunction passing through zero is known as a null contour line.

For the two shape eigenfunctions corresponding to the first pair of multiple prompt eigenvalues, the observed convergence to the shapes predicted by one-speed diffusion theory is slow (Figure 6.3). With a tenfold increase in the number of particle histories in run $\mathrm{C}$, this pair of eigenvalues converges to the same value. But, the eigenfunctions predicted by diffusion theory have null contour lines along $x=0$ and $y=0$, not 


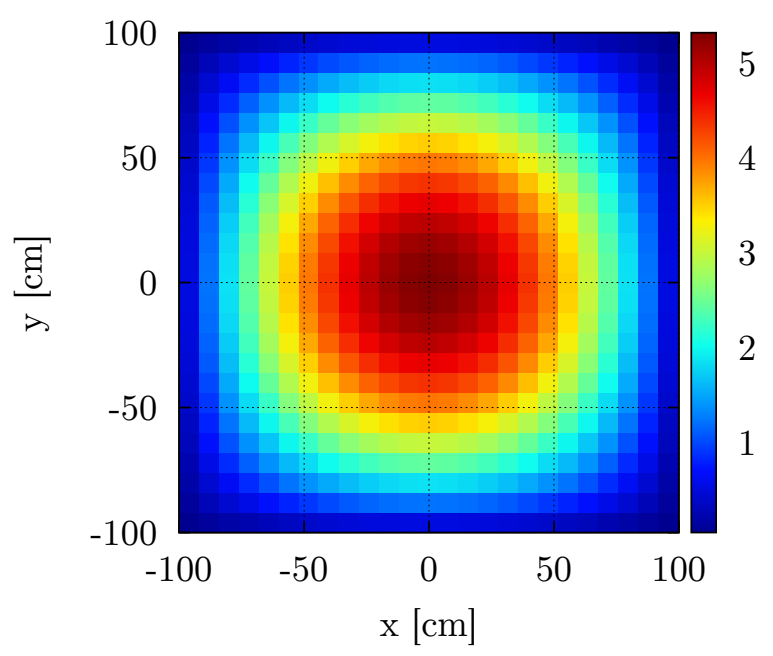

Figure 6.2: The two-dimensional, prompt fundamental shape eigenfunction of the hydrogenous cube has a smooth, symmetric cosine shape. Axially, this eigenfunction has an assumed cosine shape. This normalized eigenfunction corresponds to the thermal energy interval, $g=3$, but the eigenfunctions corresponding to the other energy intervals have the same shape.

the $\theta=30^{\circ}$ and $\theta=120^{\circ}$ null contours delivered by the TRMM-calculated shape eigenfunctions. In fact, the null contour line for the second shape eigenfunction calculated with fewer particle histories is more aligned with $x=0$.

Physically, these two shape eigenfunctions correspond to kinetic modes that capture complementary contributions to the expanded flux solution that decay at the same rate. For example, if an initial source is on the null contour line of the first shape eigenfunction, than the contribution from the corresponding kinetic mode is negligible, but the contribution from the second kinetic mode is non-zero. The contribution from both of these kinetic modes is negligible for a central source. The net contribution of these two kinetic modes is more important to the expanded solution than the individual contribution or behavior of either shape eigenfunction. This leads to the conclusion that it is important that the eigenfunctions have perpendicular null contours: the shape eigenfunctions calculated with more particle histories represent a better estimate than those calculated with fewer particle histories, whose null contours are not perpendicular. Also consider that the alignment of the null contours predicted by diffusion theory along $x=0$ and $y=0$ is a direct result of the rectangular geometry: if the problem boundary is a circle, there is no definitive way of determining where a null contour should cross the geometry, as long as the two shape 
$\alpha=-2785 \mathrm{~s}^{-1}$

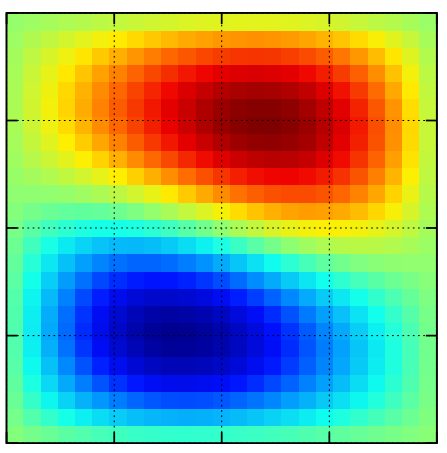

$\alpha=-2787 \mathrm{~s}^{-1}$

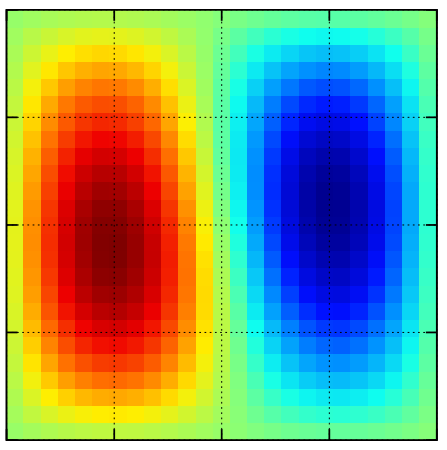

Mode 1

$\alpha=-2788 \mathrm{~s}^{-1}$

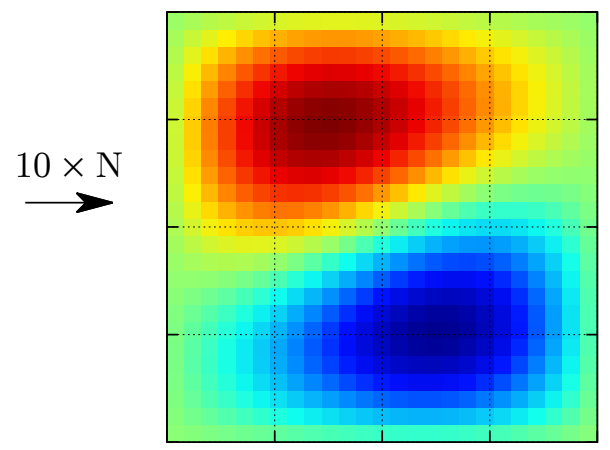

Mode 2

$$
\alpha=-2788 \mathrm{~s}^{-1}
$$

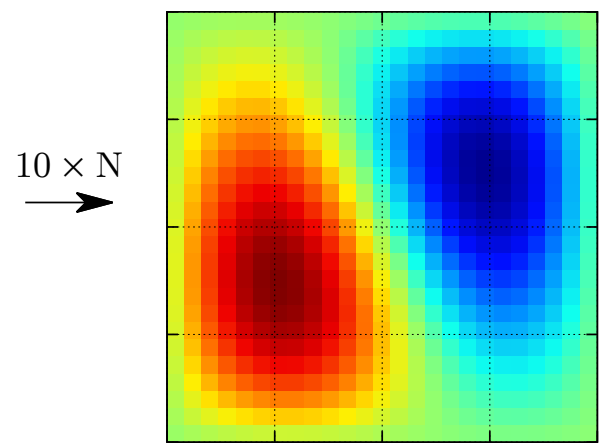

Figure 6.3: As OpenTRMM runs more particle histories for the hydrogenous cube, the first pair of multiple $\alpha$ eigenvalues converges as their corresponding shape eigenfunctions are still approaching the symmetric shapes predicted by diffusion theory. The $x$ and $y$ dimensions are the same as shown for the fundamental mode, the normalized shape eigenfunctions correspond to the thermal energy interval, $g=3$, and the results are from runs B (left) and C (right).

eigenfunctions are perpendicular.

While these skewed shape eigenfunctions have little effect on expanded flux solutions, it is still incorrect to individually present these shape eigenfunctions as true. In the limit as the number of particle histories becomes large, $N \rightarrow \infty$, the first two shape eigenfunctions are expected to have more symmetric shapes. But, the fundamental shape eigenfunctions and many of the first few higher eigenfunctions calculated from runs B and C are nearly identical. One-speed diffusion theory from Eq. (6.6) is unable to predict many of these higher shape eigenfunctions (Figure 6.4). Only a few higher shape eigenfunctions exhibit the slow convergence behavior, primarily a few of the asymmetric shape eigenfunctions. In the application of the fission 
Mode 3

$\alpha=-4969 \mathrm{~s}^{-1}$

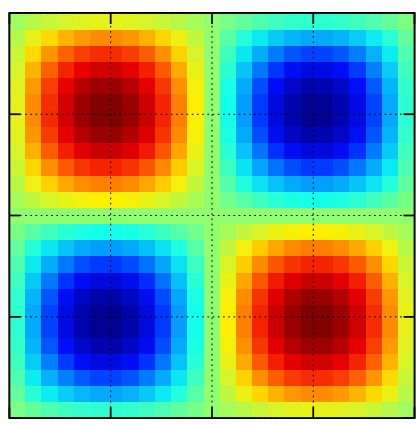

Mode 10

$\alpha=-11809 \mathrm{~s}^{-1}$

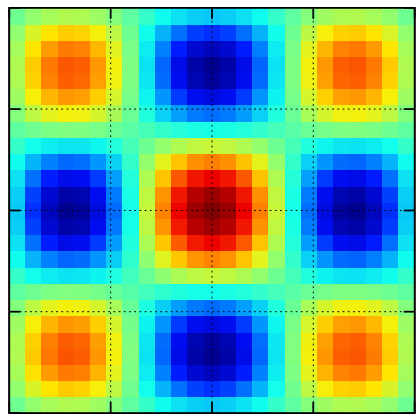

Mode 4

$\alpha=-6627 \mathrm{~s}^{-1}$

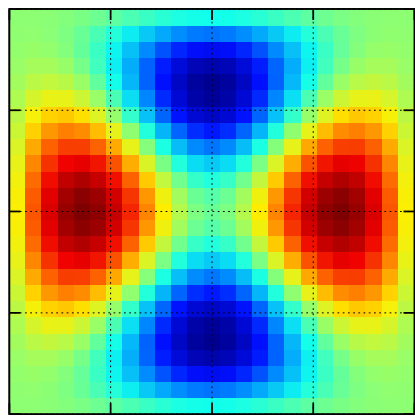

Mode 11

$\alpha=-13108 \mathrm{~s}^{-1}$

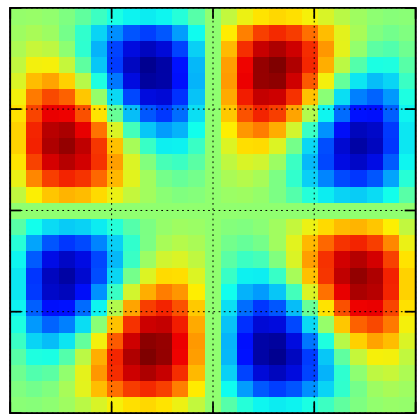

Mode 5

$\alpha=-6631 \mathrm{~s}^{-1}$

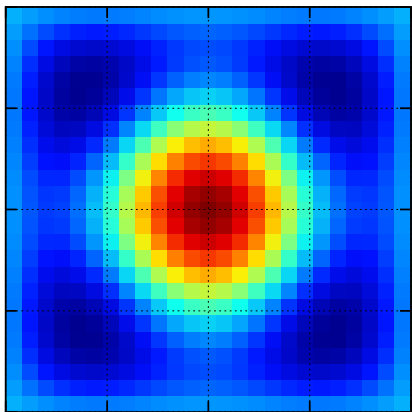

Mode 12

$\alpha=-13114 \mathrm{~s}^{-1}$

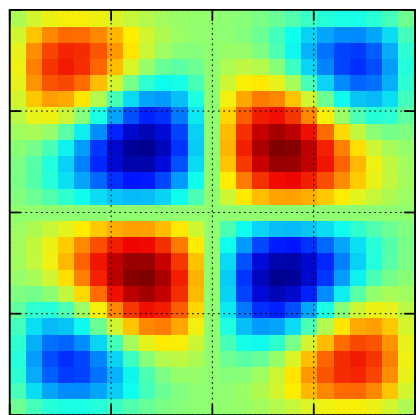

Figure 6.4: Selected shape eigenfunctions of the hydrogenous cube demonstrate the progression of the first few prompt shape eigenfunctions. All eigenfunctions have positive and negative parts, with contour lines identified in light green. The $x$ and $y$ dimensions are the same as shown for the fundamental mode, the normalized shape eigenfunctions correspond to the thermal energy interval, $g=3$, and the results are from run $\mathrm{C}$.

matrix via Monte Carlo methods, a similar slow convergence behavior is observed for a few higher shape eigenfunctions [6], where rotations of the $k$ eigenfunctions occurs as the Monte Carlo uses more particle histories. These observations suggest that Monte Carlo statistics have a larger effect on the calculation of particular shape eigenfunctions. Also, the definition of the position phase space with a single, large axial position interval may contribute to this slow convergence behavior.

\subsubsection{Eigenfunction Expansion}

To demonstrate the application of the eigenfunction expansion, consider detectors within each position interval that respond mostly to thermal neutrons, but also to fast 
neutrons with a smaller fractional probability (Table 6.4). Then, use the expanded flux solution to calculate detector responses from an initial isotropic, high-energy pulse source. This delivers the relative expected two-dimensional response throughout the system at any given time (Figure 6.5). Then, using the expanded flux solution to yield the responses of these detectors from an initial isotropic, high-energy pulse source delivers the relative expected two-dimensional response throughout the system at any given time (Figure 6.5).

At $t=2 \mu \mathrm{s}$, a sharp detector response peak occurs in the position interval at the location of the initial source. High-energy neutrons collide with water in the surrounding position intervals, causing a smooth decrease in the small region surrounding the initial source. Elsewhere, the relative detector response is nearly zero because at this time, only high-energy neutrons from the initial source populate the entire problem. At $t=120 \mu \mathrm{s}$, the relative detector response takes on a much smoother shape as neutrons slow down to thermal energies in many of the regions surrounding the initial source. The peak is still located in the region of the initial source. At $t=520 \mu \mathrm{s}$, the relative detector response shape begins to assume a cosine shape as the peak shifts towards the center of the cube and thermal neutrons populate the entire cube. At $t=50 \mathrm{~ms}$, the relative detector response shows the contribution only from the delayed kinetic modes, as all prompt kinetic modes are decayed. This shape is only slightly different than the prompt kinetic fundamental mode due to kinetic distortion. The emission of neutrons from delayed neutron precursors is the only source remaining at this time. The prompt fundamental kinetic mode dominates the detector response shape from approximately $t=2$ to $t=15 \mathrm{~ms}$.

The relative detector response shape is more convenient for visualization because the actual detector response varies several orders of magnitude over the course of the transient. An alternate visualization shows the time-dependent detector responses of two detectors placed in separate position intervals within the cube (Figure 6.6): one detector close to the initial source and another on the opposite side of the cube. As expected, these detectors experience very different response shapes: the nearby detector peaks above the response curve predicted by the fundamental shape eigenfunction, while the detector on the opposite side of the cube approaches the fundamental ap-

Table 6.4: Simulated cross sections for the hydrogenous cube.

\begin{tabular}{cccc}
\hline$g$ & 1 & 2 & 3 \\
\hline$\Sigma_{d g}$ & 0.0045 & 0.1030 & 0.8925 \\
\hline
\end{tabular}


proximation from below. On a log-log scale, this difference is more noticeable: the short-time response of the nearby detector is over four orders of magnitude greater than that of the detector on the opposite side of the cube reacting to the same pulse

$$
\mathrm{t}=2 \mu \mathrm{s}
$$

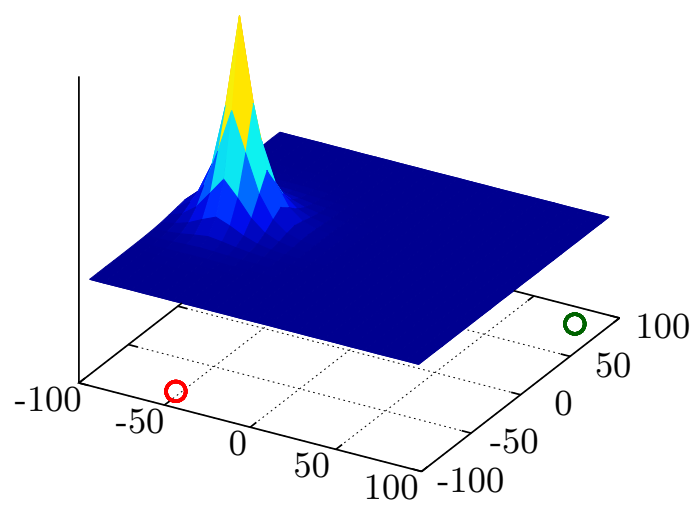

$$
\mathrm{t}=520 \mu \mathrm{s}
$$

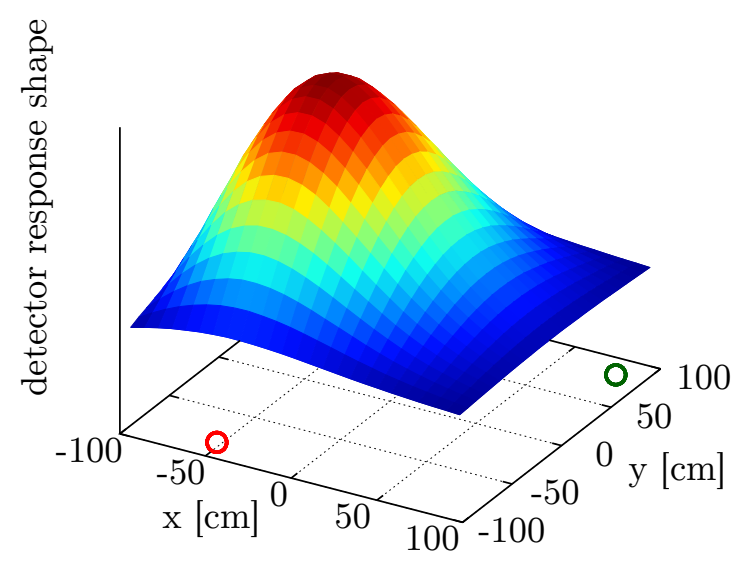

$$
\mathrm{t}=120 \mu \mathrm{s}
$$

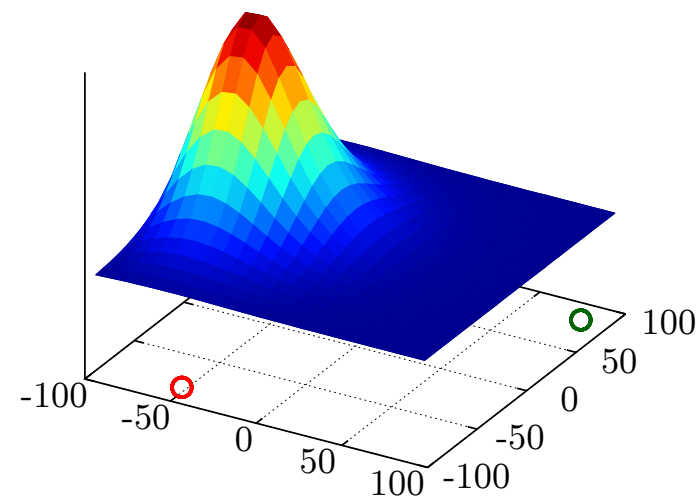

$$
\mathrm{t}=50 \mathrm{~ms}
$$

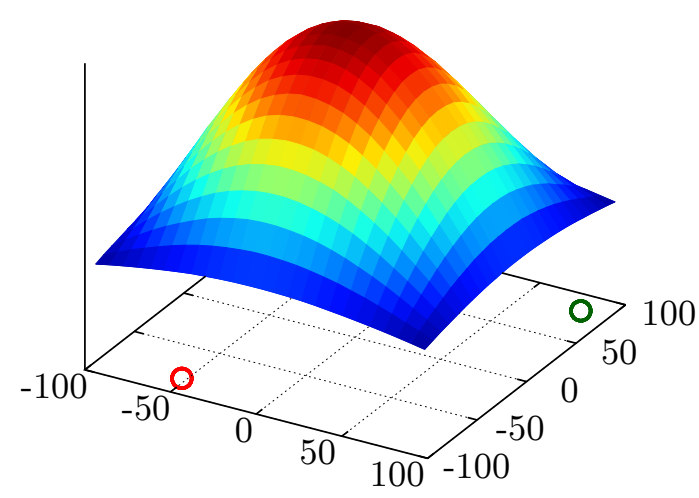

Figure 6.5: Snapshots of the relative detector response shape calculated from the expanded flux solution for the hydrogenous cube demonstrate the application of the TRMM in simulating time-dependent solutions. There is no TDMC solution for verification. OpenTRMM uses the run $\mathrm{C}$ phase space discretization, and defines the initial source as a pulse at $t=0$ with strength $Q_{0}=1 \times 10^{10} \mathrm{~cm}^{-3} \cdot \mathrm{s}^{-1}$ and no initial precursor concentrations. The source is in the highest energy interval $g=1$ in the $n=113$ position interval distributed equally in all $M$ directions. This source design approximates a $E=14.1 \mathrm{MeV}$ pulse in the fuel region at $(x, y)=(-64,0) \mathrm{cm}$. The eigenfunction expansion uses all $N G M+N_{f} J=18,750$ kinetic modes in the expansion. For the detector response plots, the locations of detector 1 and 2 are circled in red and green, respectively. 


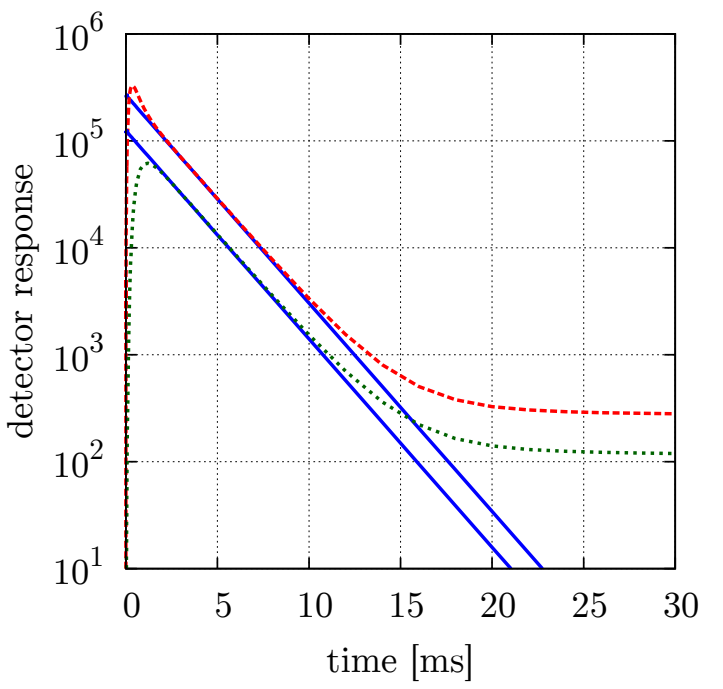

prompt fundamental

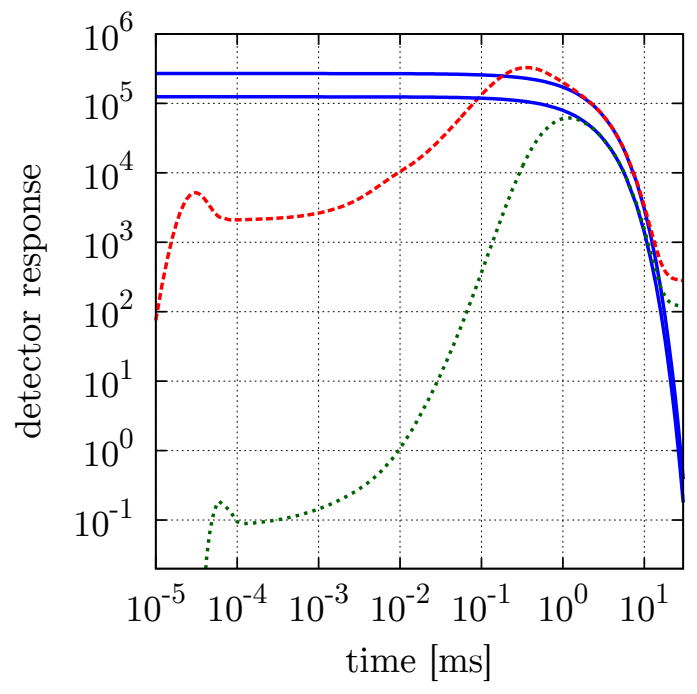

detector 2 …........

Figure 6.6: Responses of ideal detectors in two locations of the hydrogenous cube show the strong spatial dependence of the flux. The left plot is like a typical detector response obtained during a pulsed-neutron experiment and the right plot is scaled to show the shorttime effect of the high-energy pulse. These are combinations of the expanded flux solutions in position intervals $n=153$ and $n=573$ and a simulated detector cross section. Also shown is the ideal detector responses at each location obtained with the expansion using only the prompt fundamental mode (blue lines). The selection of these position intervals approximates detector 1 at $(x, y)=(-48,-80) \mathrm{cm}$ and detector 2 at $(x, y)=(80,80) \mathrm{cm}$.

source. The smaller early response peak from high-energy source neutrons identifies the detector closer to the initial source: the peak occurs at a slightly earlier time in the nearby detector than the detector on the opposite side of the cube. In both detector responses, there is only approximately $10 \mathrm{~ms}$ from which measurements may yield an estimate of the prompt fundamental $\alpha$ eigenvalue, and the prompt fundamental approximation is a very poor representation of the actual detector responses.

\subsection{The Fast Burst Reactor CALIBAN}

Problem 19: This is an approximate OpenMC model of the small, cylindrical, metallic fast burst reactor CALIBAN [7]. The simplified geometry explicitly models the control rods, excursion rod, and binding screws (Figure 6.7) with two material compositions representing the structural and fuel materials [8] (Table 6.5). This problem is the polar opposite of the hydrogenous cube: it has a hard energy spectrum, has little neutron thermalization, is very near critical, and has a large leakage probability. Available measurements for CALIBAN include the prompt fundamental $\alpha$ eigenvalue for the critical and two subcritical configurations, and the inferred effective delayed 


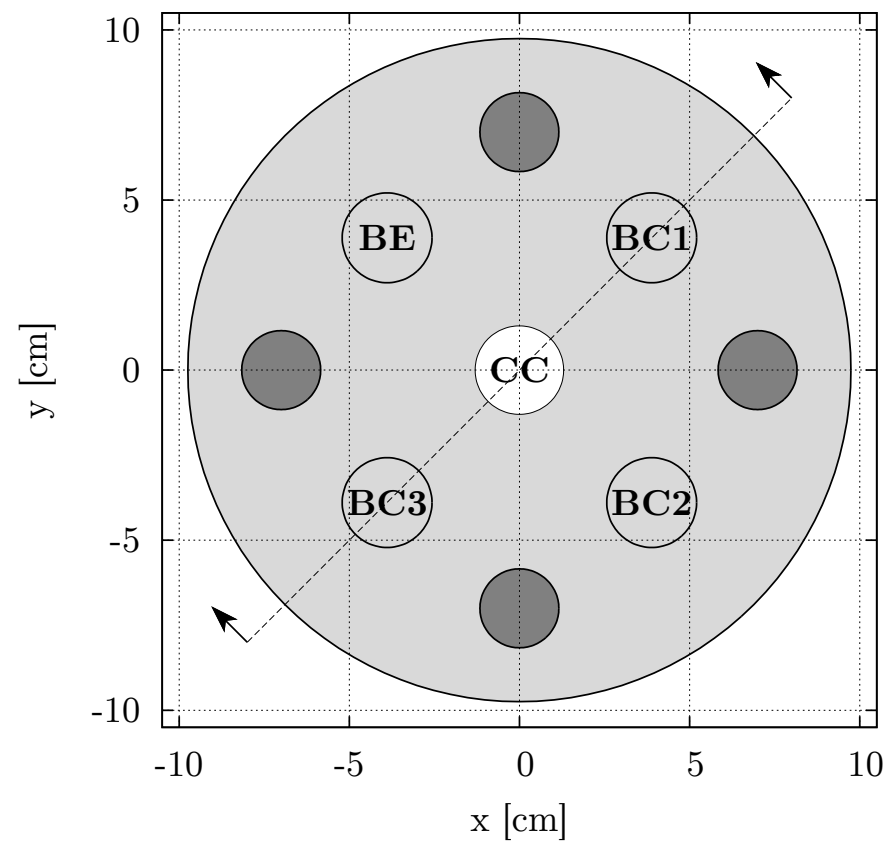

Figure 6.7: A radial slice at the center of the approximate OpenMC model of the fast burst reactor CALIBAN shows the three control and excursion rods (light gray), four metal binding screws (dark gray), and the central irradiation channel. The control rods are labeled $\mathrm{BC} 1, \mathrm{BC} 2$, and $\mathrm{BC} 3$, and the excursion rod is labeled BE. Vacuum boundaries at the extent of the cylinder are at radius $R=9.743 \mathrm{~cm}$ and height $H=25.066 \mathrm{~cm}$. The dashed line marks the section line used for showing the axial dependence of the shape eigenfunctions.

neutron fraction $\beta_{\text {eff }}[5]$.

The control and excursion rods are composed of fuel material and are inserted upward from the bottom of the core to achieve criticality. Experimental procedure inserts the $\mathrm{BE}$ rod up to $12.74 \mathrm{~cm}$, the $\mathrm{BC} 1 \operatorname{rod}$ up to $20.0 \mathrm{~cm}$, the $\mathrm{BC} 2$ rod up to $4.46 \mathrm{~cm}$, and the BC3 rod up to $12.86 \mathrm{~cm}$. This configuration is near delayed critical, at which point additional insertion of the BC3 control rod adds desired reactivity to the assembly [7].

Table 6.5: Nuclear data for CALIBAN.

\begin{tabular}{|c|c|c|c|c|c|c|c|}
\hline \multirow{2}{*}{ Material } & \multirow{2}{*}{$\rho\left[\mathrm{g} / \mathrm{cm}^{-3}\right]$} & \multicolumn{5}{|c|}{ Number Ratios } & \multirow{2}{*}{${ }^{235} \mathrm{U}$ [atom \% } \\
\hline & & $\mathrm{Fe}$ & $\mathrm{Ni}$ & Co & $\mathrm{Mo}^{\mathrm{a}}$ & $\mathrm{U}$ & \\
\hline Fuel & 17.04 & 0 & 0 & 0 & 3.67 & 1 & 93.47 \\
\hline Screws & 7.90 & 15.43 & 4.07 & 1.82 & 1 & 0 & - \\
\hline
\end{tabular}

${ }^{\text {a }}$ Natural molybdenum. 


\subsubsection{Eigenvalue Spectrum}

Initial runs with small discretized phase spaces show discrepancies between the OpenMC-calculated $k$-effective and the sign of the calculated fundamental $\alpha$ eigenvalue from the TRM. While this discrepancy is well within $\beta_{\text {eff }}$, it is significant considering the proximity of the assembly to critical. Some contributing factors to this discrepancy is the higher energy spectrum and large fluctuations due to the sampling of delayed neutron precursors: thermal configurations do not show similar discrepancies.

\section{Approach to Prompt Critical}

For different BC3 insertions, OpenTRMM divides the position phase space in a $r$ $\theta-z$ geometry, instead of the regular Cartesian geometry for the hydrogenous cube. The smaller size of the phase space allows for the use of a direct eigenvalue solver

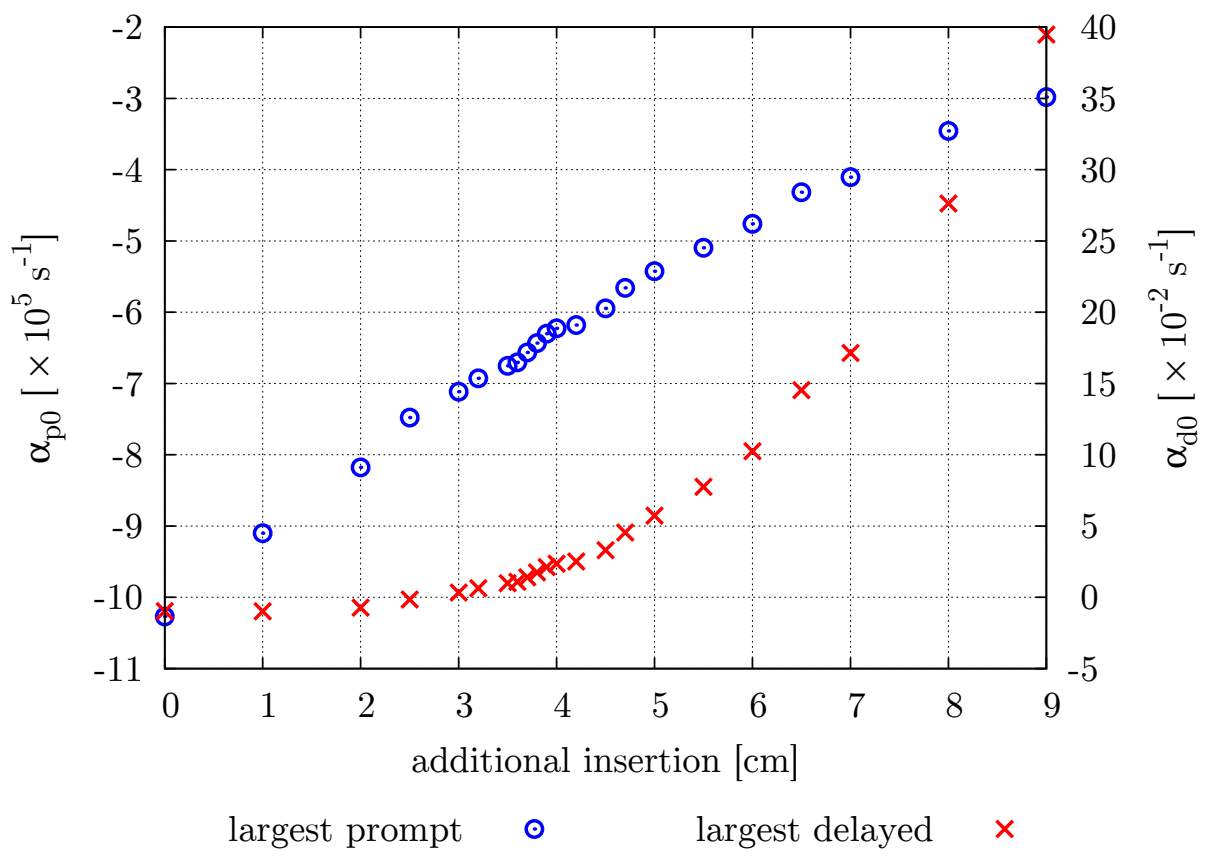

Figure 6.8: The trend of the largest delayed and prompt $\alpha$ eigenvalues with additional insertions of the BC3 control rod show the transition point at which the TRMM predicts the CALIBAN model switching from a subcritical to delayed supercritical state. The BC3 rod is already inserted to $12.86 \mathrm{~cm}$ before the additional insertion, and the model is still in a delayed supercritical state at full insertion of the BC3 rod. OpenTRMM uses $N=256$ position, $G=3$ energy, and $M=8$ direction intervals to tally the TRM. The position intervals distribute among the $r-\theta-z$ geometry: there are eight radial intervals, four azimuthal intervals, and eight axial intervals. 
to determine the entire $\alpha$-eigenvalue spectrum. Tracking the largest prompt and delayed $\alpha$ eigenvalues (Figure 6.8) show the BC3 insertion that causes the system to switch from a subcritical to a delayed supercritical state. This transition occurs at approximately $2.7 \mathrm{~cm}$ of additional insertion. The largest prompt $\alpha$ eigenvalue linearly decreases in magnitude as the control rod is inserted, while the largest delayed $\alpha$ eigenvalue increases rapidly after the model crosses into the delayed supercritical state. Even near full insertion of the rod the magnitude of this delayed $\alpha$ eigenvalue dwarfs that of the largest prompt eigenvalue.

Tracking these two eigenvalues while continuing to add reactivity to the system via insertion of the BC2 control rod shows the insertion that causes the system to switch from delayed supercritical to prompt supercritical (Figure 6.9). This transition occurs at approximately $4 \mathrm{~cm}$ of additional insertion. The prompt $\alpha$ eigenvalue continues to trend linearly through both the delayed and prompt supercritical states, while the delayed $\alpha$ eigenvalue nearly asymptotically approaches the transition point, after which it returns to a constant negative value near the decay constant of the longestlived delayed neutron precursor group. Very near the transition point, OpenTRMM delivers a decreased delayed and prompt $\alpha$ eigenvalue.

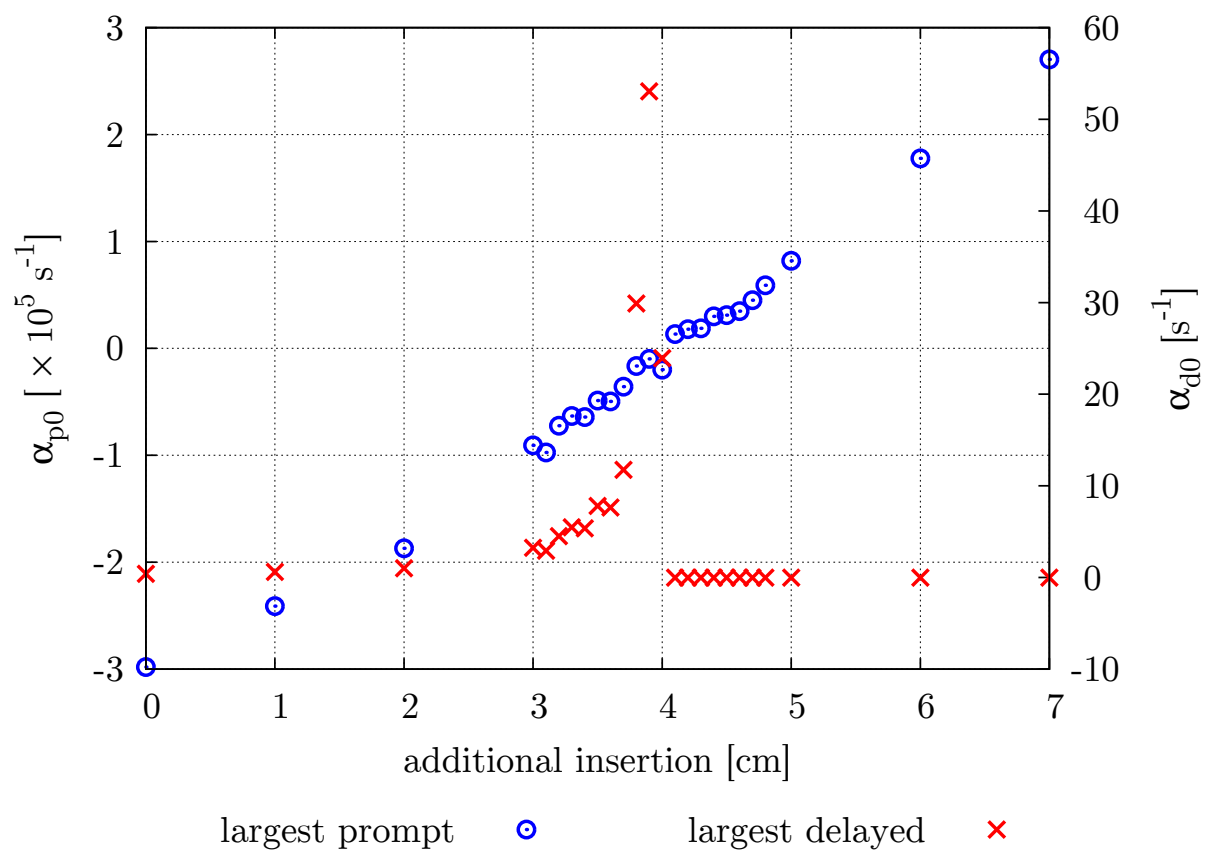

Figure 6.9: The trend of the largest delayed and prompt $\alpha$ eigenvalue with additional insertion of the $\mathrm{BC} 2$ control rod show the transition point at which the TRMM predicts the CALIBAN model switching from a delayed supercritical to prompt supercritical state. The BC3 rod is inserted to $21.86 \mathrm{~cm}$ and the initial position of the $\mathrm{BC} 2 \operatorname{rod}$ is $4.46 \mathrm{~cm}$. 
For all runs with different control rod insertions, a significant change only occurs for the largest prompt and delayed $\alpha$ eigenvalues. The remaining calculated delayed eigenvalues show no trend with control rod insertion. The first few prompt eigenvalues decrease in magnitude, i.e., become less negative, with the additional reactivity insertion. Relative to the calculated eigenvalue, this trend is very small: from the subcritical case with no additional insertion to the most supercritical case, the second, third, and fourth eigenvalues increase by $2.2 \%, 1.2 \%$, and $0.8 \%$, respectively. This decreasing trend applies the the next few eigenvalues, until there is no discernible relative change in the calculated eigenvalues.

\section{Comparisons to Measured Data}

As calculated by OpenTRMM, the effective delayed neutron fraction is $640 \pm 8 \mathrm{pcm}$, close to the $633 \pm 7 \%( \pm 40)$ pcm inferred from measurements with the Nelson number method [9]. However, the prompt fundamental eigenvalue shows more disagreement, underestimating the measured prompt fundamental eigenvalue by nearly $10 \%$ for the critical configuration of CALIBAN [5]. There are no known calculations of the fundamental $\alpha$ eigenvalue of CALIBAN.

\section{Discretizing the Phase Space}

The number of position intervals $N$ is kept large relative to the number of energy and direction intervals. This is because the first few shape eigenfunctions have interesting geometric dependences that are negated with some position interval approximations, as shown in the hydrogenous cube problem. Thus, a larger number of position intervals is more important than energy or direction intervals, and the position phase space is discretized further: divisions along a Cartesian $15 \times 15 \times 15$ grid make up $N=3015$ position intervals within the cylindrical geometry of the CALIBAN model. With the size of the TRM for this phase space discretization, it is necessary to determine the eigenvalues iteratively, and only a few prompt $\alpha$ eigenvalues are obtained. Results yield the same fundamental $\alpha$ eigenvalues as the previous runs within $0.5 \%$, while higher $\alpha$ eigenvalues show less agreement: the coarser chosen spatial discretization is having a negative effect on the higher shape eigenfunctions.

To test the effect of the discretization of the hard energy spectrum, OpenTRMM increases the number of energy intervals to $G=12$, yielding a less than $1 \%$ decrease in magnitude of the prompt fundamental $\alpha$ eigenvalue. Runs with an increased number of direction intervals $M=16$ show a $1.4 \%$ decrease in magnitude of the prompt 
fundamental eigenvalue. OpenTRMM also runs problems with a large number of position intervals in the discretized $r-\theta-z$ phase space, with similar trends. But, with more $\theta$ intervals, the number of direction intervals becomes more important because of the shape of the volumes. Also, this discretization has a greater effect on the higher $\alpha$ eigenvalues, and the regular cubic lattice of position intervals delivers better results.

\section{Comparisons to Diffusion Theory}

As with the hydrogenous cube, one-speed diffusion theory gives some predictions on the progression of the first few prompt $\alpha$ eigenvalues, defined in Eq. (6.1), where

$$
B_{p}^{2}=\left(\frac{j_{i_{\theta}, i_{r}}^{2}}{\tilde{R}}\right)^{2}+\left(\frac{i_{z} \pi}{\tilde{H}}\right)^{2}
$$

where $j_{i_{\theta}, i_{r}}$ is root $i_{r}$ of the $i_{\theta}$ th Bessel function $J_{i_{\theta}}, \tilde{R}$ and $\tilde{H}$ is the extrapolated radius and height, and $\left(i_{z}, i_{\theta}, i_{r}\right)$ is the triplet of integers identifying the eigenvalue. Assuming zero extrapolated boundaries, i.e., $\tilde{H}=H$ and $\tilde{R}=R$, and $k_{\infty}=2.2$ for this critical system, the first few ratios computed align with those calculated by OpenTRMM (Table 6.6). The worst agreement is for the $i_{z}=3$ eigenvalues, where the $(3,0,1)$ eigenvalue is predicted to be smaller in magnitude than the $(2,1,1)$ eigenvalue: this implies that the ratio of $\tilde{H}$ to $\tilde{R}$ used in one-speed diffusion theory is not exact. Other than these eigenvalues, diffusion theory predicts the order of the prompt $\alpha$ eigenvalues.

Table 6.6: The first few calculated prompt $\alpha$ eigenvalues of the OpenMC CALIBAN model.

\begin{tabular}{rrrrr}
\hline$n^{\mathrm{a}}$ & $\left(i_{z}, i_{\theta}, i_{r}\right)$ & \multicolumn{2}{c}{ OpenTRMM $^{\mathrm{b}}$} & ${\frac{\alpha_{p n}}{{ }^{\mathrm{c}}}}^{\alpha_{p 0}}$ \\
\hline 0 & $(1,0,1)$ & -6.453 & 1.0 & 1.0 \\
1 & $(2,0,1)$ & -396.7 & 61.4 & 52.1 \\
2 & $(1,1,1)$ & -683.7 & 105.9 & 102.7 \\
3 & $(1,1,1)$ & -694.8 & 107.7 & 102.7 \\
4 & $(2,1,1)$ & -978.9 & 151.7 & 153.8 \\
5 & $(2,1,1)$ & -994.0 & 154.0 & 153.8 \\
6 & $(3,0,1)$ & -1057.6 & 163.9 & 137.3 \\
7 & $(1,2,1)$ & -1315.8 & 203.9 & 236.2 \\
8 & $(3,1,1)$ & -1459.4 & 226.2 & 238.9 \\
\hline
\end{tabular}

\footnotetext{
${ }^{a}$ As ordered by the calculated ratio.

b $N=3015, G=3$, and $M=8$.

c Ratios from one-speed diffusion theory.
} 


\subsubsection{Shape Eigenfunctions}

As with the hydrogenous cube, one-speed diffusion theory predicts the general progression of the prompt shape eigenfunctions, assuming that this is a homogeneous medium, following as

$$
\phi(x, y, z)=J_{i_{\theta}}\left(j_{i_{\theta}, i_{r}} \frac{r}{\tilde{R}}\right) \sin \left(i_{z} \pi \frac{z}{\tilde{H}}\right) \cos \left(i_{\theta} \theta\right)
$$

These shape eigenfunctions are easily identifiable by examination of the OpenTRMMcalculated eigenfunctions in the three dimensional position phase space. A shape eigenfunction has $i_{z}-1$ axial, $i_{\theta}$ azimuthal, and $i_{r}-1$ null contour lines, outside of those at the edges of the geometry.

\section{The First Few Prompt Eigenfunctions}

For example, the calculated prompt fundamental shape eigenfunction plotted in $r$ $\theta$ geometry is all-positive and has a $J_{0}$ Bessel function shape in the radial position phase space (Figure 6.10), so $i_{r}=1$ and $i_{\theta}=0$ This shape eigenfunction decreases at

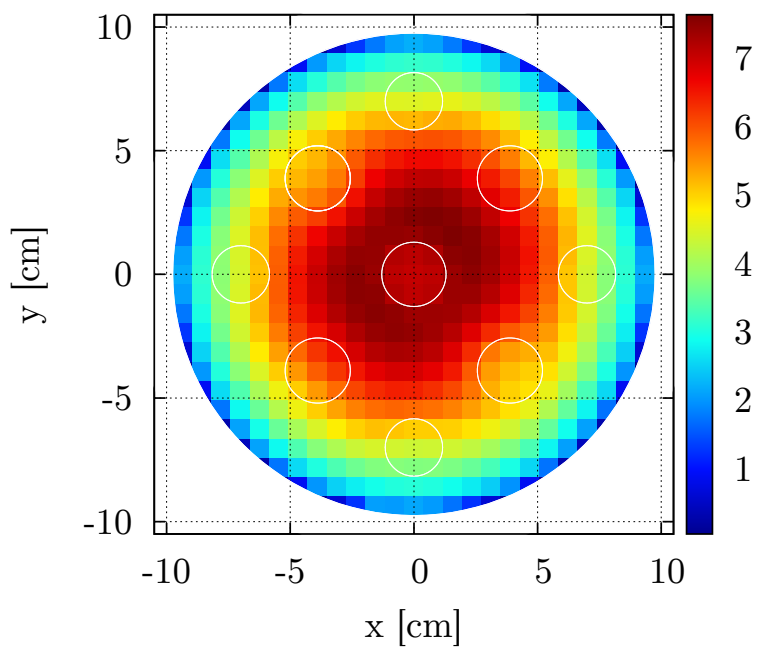

Figure 6.10: An $r-\theta$ plot of the calculated fundamental prompt shape eigenfunction of the OpenMC CALIBAN model shows the Bessel function shape with no azimuthal variation. This is shown at the center of the model, approximately $z=12.5 \mathrm{~cm}$. This normalized eigenfunction corresponds to the lowest energy interval, $g=3$, but the eigenfunctions corresponding to the other energy intervals have the same shape. OpenTRMM uses $N=$ 3198 position and $M=8$ direction intervals and white circles show the physical location of features in the CALIBAN geometry. 
$(x, y)=(0,0)$ due to the central void channel, and is relatively flat across the position intervals containing the four metal screws. Also, the shape is slightly asymmetric, decreasing more in the region containing the $\mathrm{BC} 2$ rod due to the large void left above the inserted rod. The shape eigenfunction is at its maximum at the center of the CALIBAN model. With this limited information, it is unclear if this is the prompt fundamental shape eigenfunction, or any of the eigenfunctions thereafter corresponding to odd $i_{z}$, as all those shape eigenfunctions are also maximized at the center of the model. Examination in $r$ - $z$ geometry shows the calculated prompt fundamental shape eigenfunction has an all-positive sine shape (Figure 6.11), so $i_{z}=1$. This section cuts through the $\mathrm{BC} 3$ and $\mathrm{BC} 1$ control rods: the $\mathrm{BC} 1$ control rod is fully inserted, but there is a void above the BC3 control rod, which has the effect of decreasing the fundamental shape eigenfunction in that region, causing the asymmetric shape.

The next shape eigenfunction corresponds to the triplet $(2,0,1)$, so it has the same

Mode 0

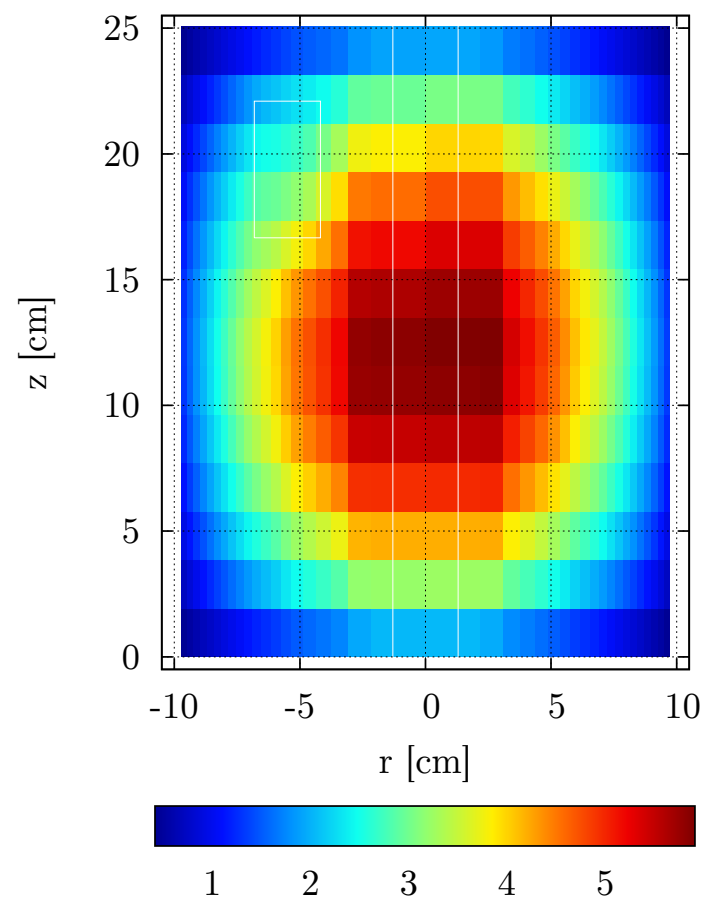

Mode 1

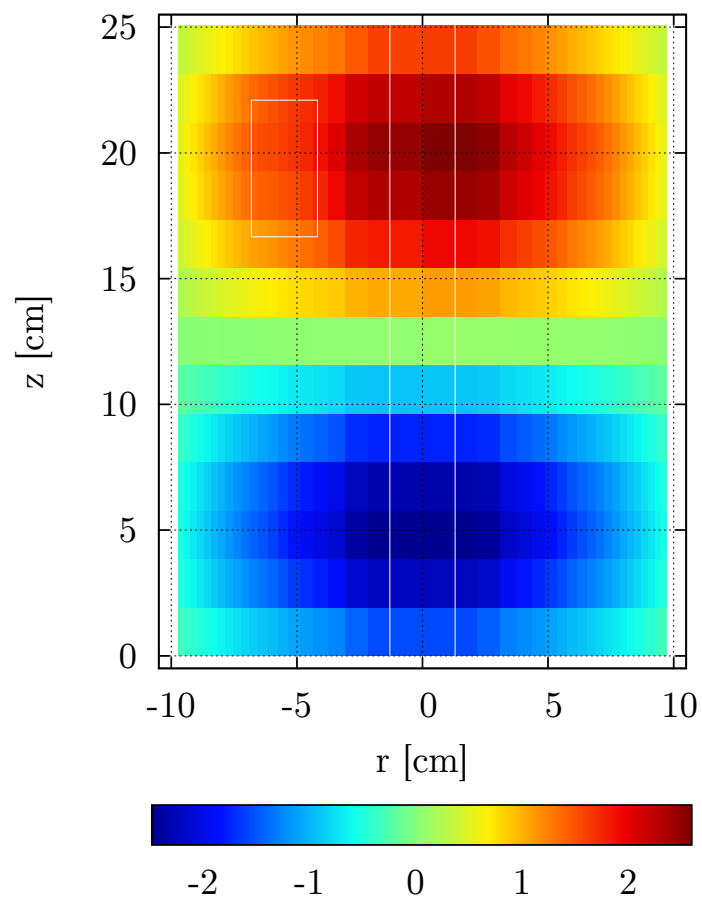

Figure 6.11: Plots in the $r-z$ space of the calculated fundamental and first prompt shape eigenfunctions shows the axial sine shape of the eigenfunctions corresponding to the $i_{z}=1$ an $i_{z}=2$ triplets. This section is taken through the BC3 (left) and BC1 (right) control rods. This normalized eigenfunction corresponds to the lowest energy interval, $g=3$, but the eigenfunctions corresponding to the other energy intervals have the same shape. OpenTRMM uses $N=3120$ position and $M=12$ direction intervals, and white lines show the physical location of voids along this section. 
basic $r-\theta$ dependence of the fundamental shape eigenfunction, but has a null contour at the center of the model near $z=12.5 \mathrm{~cm}$. This shape eigenfunction shows less effect from the void above the BC3 control rod, and like all higher shape eigenfunctions, this has positive and negative parts. Most of the few calculated eigenfunctions have axial shapes like these first two shape eigenfunctions. The shape eigenfunction corresponding to $i_{z}=3$ has two contour lines, dividing the shape eigenfunction into three distinct axial parts. This pattern continues until the number of axial position states becomes unable to describe smaller axial variations, i.e., when $i_{z}$ approaches the number of axial position states defined in the TRM. Once this occurs, confidence in the OpenTRMM-calculated eigenvalues and eigenfunctions is low, though there are not enough calculated eigenvalues to actually see these shape eigenfunctions.

Radially, the TRMM calculates some shape eigenfunctions not predicted by the diffusion theory in Eq. (6.8) associated with the $i_{\theta}$ triplet, i.e., the azimuthal variation of the shape eigenfunction. Instead of the $\cos \left(i_{\theta} \theta\right)$ azimuthal dependence, there are two perpendicular shape eigenfunctions corresponding to the $i_{\theta}=1$ triplet (Figure 6.12). These are like the first two eigenfunctions of the hydrogenous cube, and the $\alpha$ eigenvalues corresponding to these shape eigenfunctions are nearly the same. But, the next $i_{\theta}=2$ triplet has two perpendicular null contours spanning the $r-\theta$ geometry an do not show multiplicity. The shape eigenfunction corresponding to the triplet $(3,0,1)$ is nearly indistinguishable from the fundamental when observed in the $r-\theta$ geometry.

\section{Discussion}

For some experiments, suppressing these higher shape eigenfunctions is important. Minimizing contributions from the first shape eigenfunction $(2,0,1)$ is possible by locating a source axially near the center at $z=12.5 \mathrm{~cm}$ : this is done in practice [5]. But, the higher shape eigenfunctions are not easily minimized: an external source from the outer radius of CALIBAN excites the second and third shape eigenfunctions. Minimizing these two shape eigenfunctions by locating a source along the $z$ axis of CALIBAN only enlarges contributions from the axial shape eigenfunctions. Fortunately for CALIBAN, the higher shape eigenfunctions decay incredibly quickly: as calculated by OpenTRMM, the first, second, and third shape eigenfunctions decay to about $0.01 \%$ of their original contribution in $232 \mathrm{~ns}, 135 \mathrm{~ns}$, and $133 \mathrm{~ns}$, respectively. Thus, simply minimizing the first shape eigenfunction is sufficient for most measurements.

A contributing factor for the large $\alpha$ eigenvalues is the small size of CALIBAN. 
Mode 1

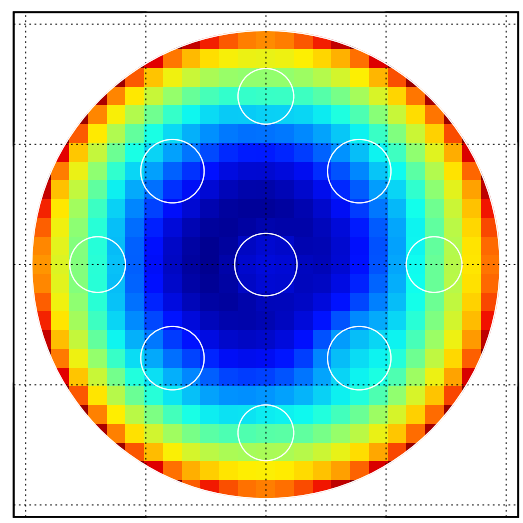

Mode 3

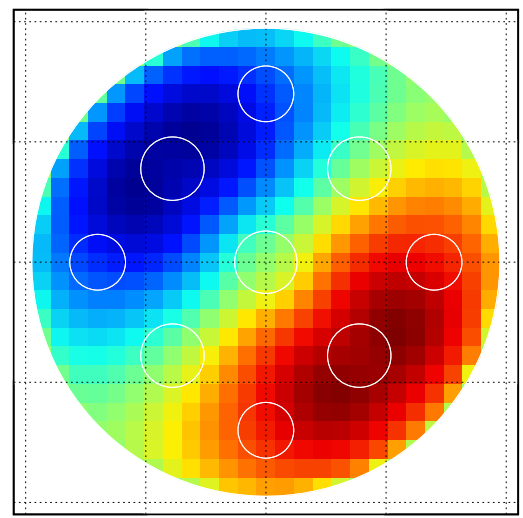

Mode 2

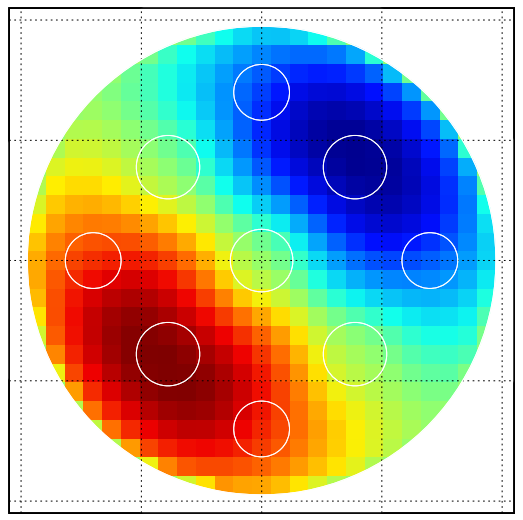

Mode 7

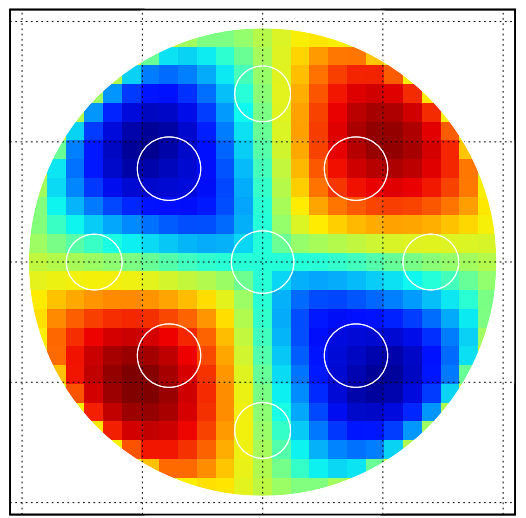

Figure 6.12: Plots in the $r-\theta$ space of selected calculated prompt shape eigenfunctions of the OpenMC CALIBAN model show the different identifiable characteristics. The first shape eigenfunction is at the bottom half of the model, at approximately $z=6.25 \mathrm{~cm}$, while all other normalized eigenfunctions are at the center of the model, approximately $z=12.5 \mathrm{~cm}$, and correspond to the lowest energy interval, $g=3$. OpenTRMM uses $N=3198$ position and $M=8$ direction intervals and the white circles show the physical location of features in the CALIBAN geometry. The dimensions are the same as in the plot of the fundamental shape eigenfunctions.

With a reduction in the enrichment, a small fast burst reactor most likely must increase in size. Assuming the same metallic density of the molybdenum and uranium alloy, and reducing the ${ }^{235} \mathrm{U}$ enrichment to $20.0 \%$ requires a cylinder that is approximately $2.2 \times$ larger in radius and height. In keeping the same height-to-radius ratios, the relative ratios of the prompt eigenvalues does not change significantly, but OpenTRMM calculates a reduction of the first few prompt eigenvalues by a factor of 4.6. Thus, the lifetimes of the first, second, and third shape eigenfunctions increase to $1.08 \mu \mathrm{s}, 560 \mathrm{~ns}$, and $559 \mathrm{~ns}$. For the reliability of pulses from a fast burst reactor 
on the $\mu$ s timescale, it is now even more important to minimize the first few prompt shape eigenfunctions.

\subsection{The Fort St. Vrain Reactor}

Problem 20: The FSV reactor is a high-temperature, gas-cooled reactor fueled with a thorium-HEU blend. The core consists of six layers of prismatic hexagonal graphite blocks, approximately $80 \mathrm{~cm}$ in height and $36 \mathrm{~cm}$ across (flat-to-flat). Thirteen fuel blends (Table 6.7) are distributed asymmetrically into 247 columns throughout the entire active core (Figure 6.13), which is buffered by a replaceable and permanent reflectors. The top and bottom reflectors are approximately 60 and $100 \mathrm{~cm}$ thick, respectively [10]. There are 37 fuel regions.

\subsubsection{OpenMC Model}

Along with the asymmetric fuel loading, uncertainties between the design and asbuilt fuel loadings, graphite boron concentrations, boron poison rods, and control rod geometry and composition makes this a very difficult reactor to model [11]. But, there is a significant amount of measured data, including pulsed neutron experiments, so it is worth pursuing. Furthermore, calculations of the homogeneous reactor modes performed on subcritical configurations of FSV [12, 13] provide some comparisons to shape eigenfunctions of the OpenTRMM calculations.

At the center of each of the fuel regions is a control block with three large holes

Table 6.7: Total weight of uranium and thorium in the FSV fuel compositions.

\begin{tabular}{cccc}
\hline No. & Uranium $[\mathrm{kg}]$ & Thorium $[\mathrm{kg}]$ & $\mathrm{U}: \mathrm{Th} \times 100$ \\
\hline 1 & 105.6 & 2905 & 3.6 \\
2 & 80.5 & 2596 & 3.1 \\
3 & 39.2 & 636 & 6.2 \\
4 & 28.9 & 544 & 5.3 \\
5 & 88.8 & 1324 & 6.7 \\
6 & 65.9 & 1158 & 5.7 \\
7 & 111.6 & 1446 & 7.7 \\
8 & 84.4 & 1287 & 6.6 \\
9 & 36.2 & 720 & 5.0 \\
10 & 25.8 & 599 & 4.3 \\
11 & 32.1 & 549 & 5.8 \\
12 & 23.7 & 474 & 5.0 \\
13 & 50.5 & 1733 & 2.9 \\
\hline
\end{tabular}




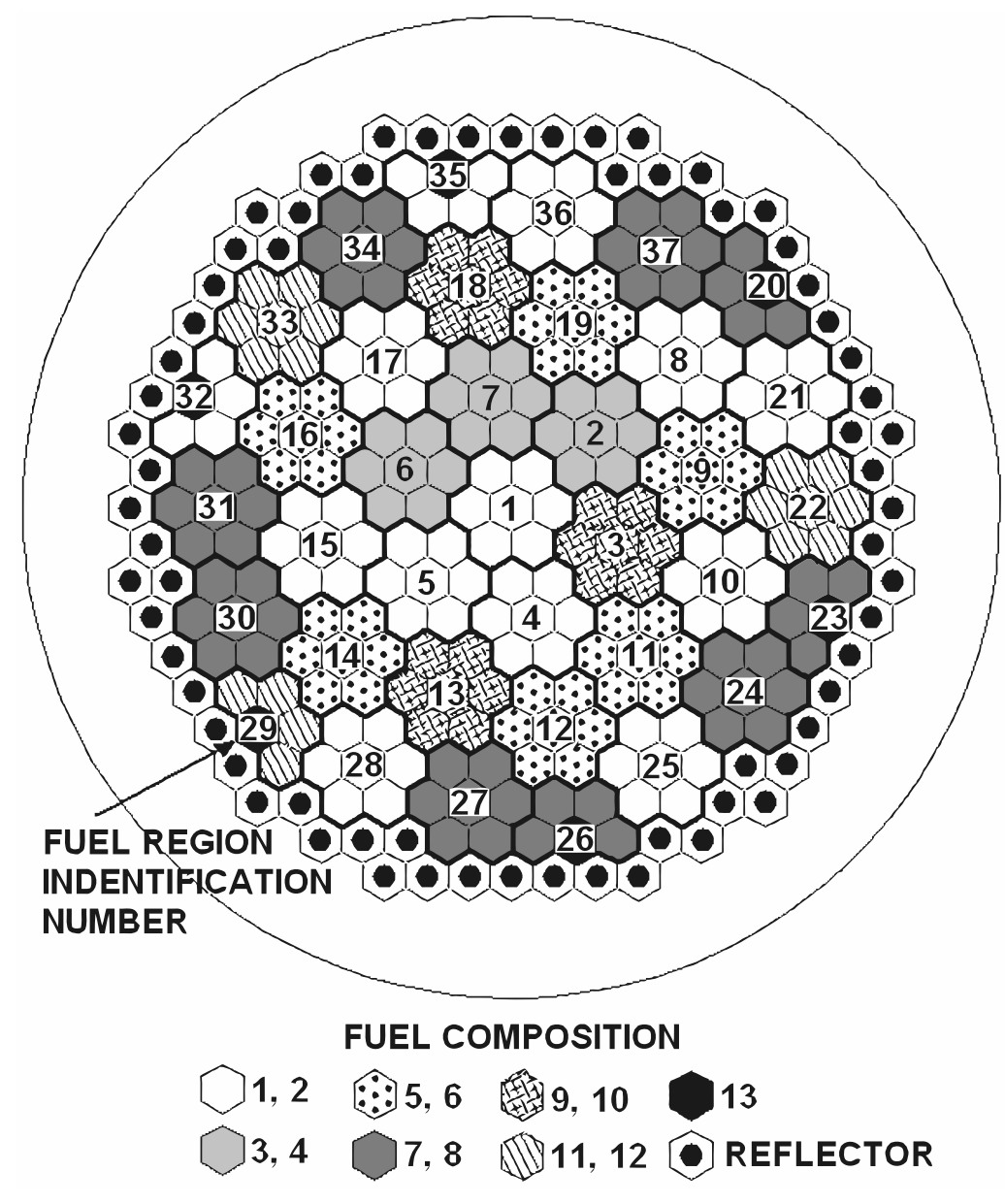

Figure 6.13: Thirteen fuel compositions of the FSV initial core are arranged asymmetrically throughout the 37 fuel regions in an effort to better approximate the equilibrium core. Odd-numbered fuel compositions fill the top three fuel blocks, and even-numbered fuel compositions fill the bottom three fuel blocks. The thirteenth fuel blend fills the central block of the six partial fuel regions at the edge of the core and the first five fuel holes of every block adjacent to the reflector region. The total outer radius of the reactor is approximately $403 \mathrm{~cm}$.

that run the length of the core: one reserve shutdown hole and two control rod channels. A control rod pair consisting of a metal spine with borated graphite runs axially through these channels. These control rods are divided into symmetric groups (Figure 6.14), and FSV operates with some of these control rods inserted.

The remainder of this section shows the OpenTRMM applied to a fully-rodded initial core, configuration number 3 and $3 \mathrm{~A}$ (Table 6.8), where the difference between these configurations is the detector location. To apply the OpenTRMM, a homogenized OpenMC model is constructed directly from as-built fuel loadings, and the position states are distributed among the 499 total individual fuel and reflector blocks of the FSV reactor. OpenTRMM uses $M=8$ direction and $G=4$ energy 


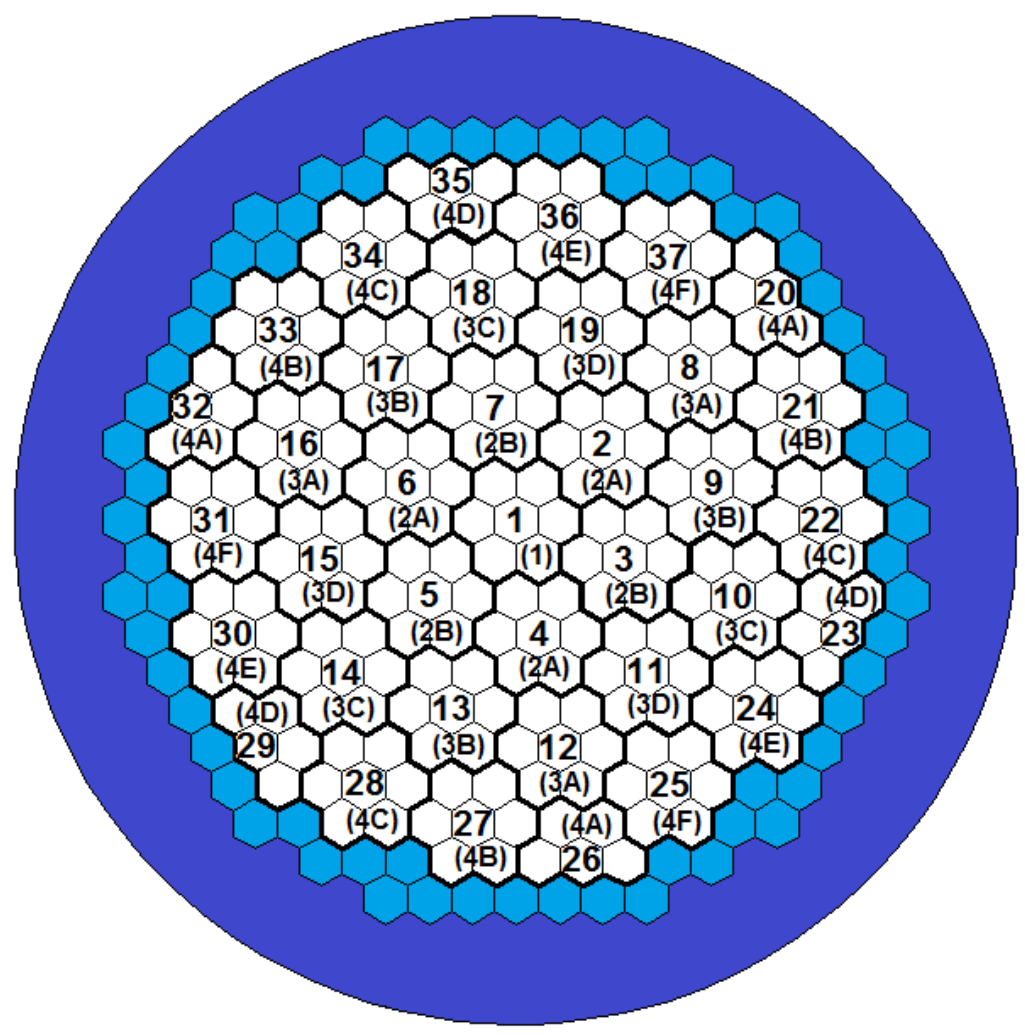

Figure 6.14: The 37 control rod pairs at the center of each fuel regions fall into thirteen symmetric groups: twelve groups each have three control rod pairs, while the control rod pair in the first fuel region is in its own separate group.

intervals.

\subsubsection{OpenTRMM Results}

For the fundamental $\alpha$ eigenvalue, OpenTRMM is able to calculate a fundamental eigenvalue of $-154.8 \mathrm{~s}^{-1}$, underestimating the $-164.6 \pm 4.9 \mathrm{~s}^{-1}$ taken from measurements. This is approximately a $6 \%$ difference from the measured value. The measured eigenvalue is an averaged value: the pulsed-neutron experiments on configurations 3 and $3 \mathrm{~A}$ yield six detector response curves. For each response curve, a least squares fit

Table 6.8: FSV pulsed-neutron experimental parameters.

\begin{tabular}{cccrrrr}
\hline \multirow{2}{*}{ Run } & \multicolumn{2}{c}{ Control Rod Configuration } & \multicolumn{4}{c}{ Axial Locations ${ }^{\text {a }}[\mathrm{cm}]$} \\
& No. & Description & $\begin{array}{r}\text { Source } \\
\text { Reg. 1 }\end{array}$ & $\begin{array}{r}\text { Detector } \\
\text { Reg. 30 }\end{array}$ & $\begin{array}{r}\text { Detector } \\
\text { Reg. 31 }\end{array}$ & $\begin{array}{r}\text { Detector } \\
\text { Reg. 16 }\end{array}$ \\
\hline 5 & 3 & \multirow{2}{*}{ All rods in } & 249 & 99 & 99 & 130 \\
6 & $3 \mathrm{~A}$ & & 249 & 69 & 69 & 99 \\
\hline a & As measured from the top core reflector interface to source/detector.
\end{tabular}

${ }^{\mathrm{a}}$ As measured from the top core reflector interface to source/detector. 
Table 6.9: FSV pulsed-neutron experiment results.

\begin{tabular}{ccccrrr}
\hline \multirow{2}{*}{ Run } & \multicolumn{2}{c}{ Control Rod Configuration } & \multicolumn{3}{c}{ Measured Prompt $\alpha^{\mathrm{a}}[\mathrm{cm}]$} \\
& No. & Description & $\begin{array}{r}\text { Detector } \\
\text { Reg. 30 }\end{array}$ & $\begin{array}{r}\text { Detector } \\
\text { Reg. 31 }\end{array}$ & $\begin{array}{r}\text { Detector } \\
\text { Reg. 16 }\end{array}$ \\
\hline 5 & 3 & \multirow{2}{*}{ All rods in } & -163.5 & -167.7 & -161.3 \\
6 & $3 \mathrm{~A}$ & & -165.0 & -158.6 & -171.7 \\
\hline
\end{tabular}

${ }^{\mathrm{a}}$ From the least squares fit of detector response.

yields an approximation to the $\alpha$ eigenvalue (Table 6.9). The single measured value reported is an average of these six measurements, which by themselves range from $-158.6 \mathrm{~s}^{-1}$ to $-171.7 \mathrm{~s}^{-1}$.

The homogeneous reactor mode expansion yields calculated estimates of the prompt fundamental thermal mode [13] that match very well to the OpenTRMMcalculated prompt fundamental shape eigenfunction (Figure 6.15). As this is a thermal shape eigenfunction, peaks occur in the reflector near the core-reflector interface. These peaks are correlated with the fuel loadings of the adjacent fuel regions: the

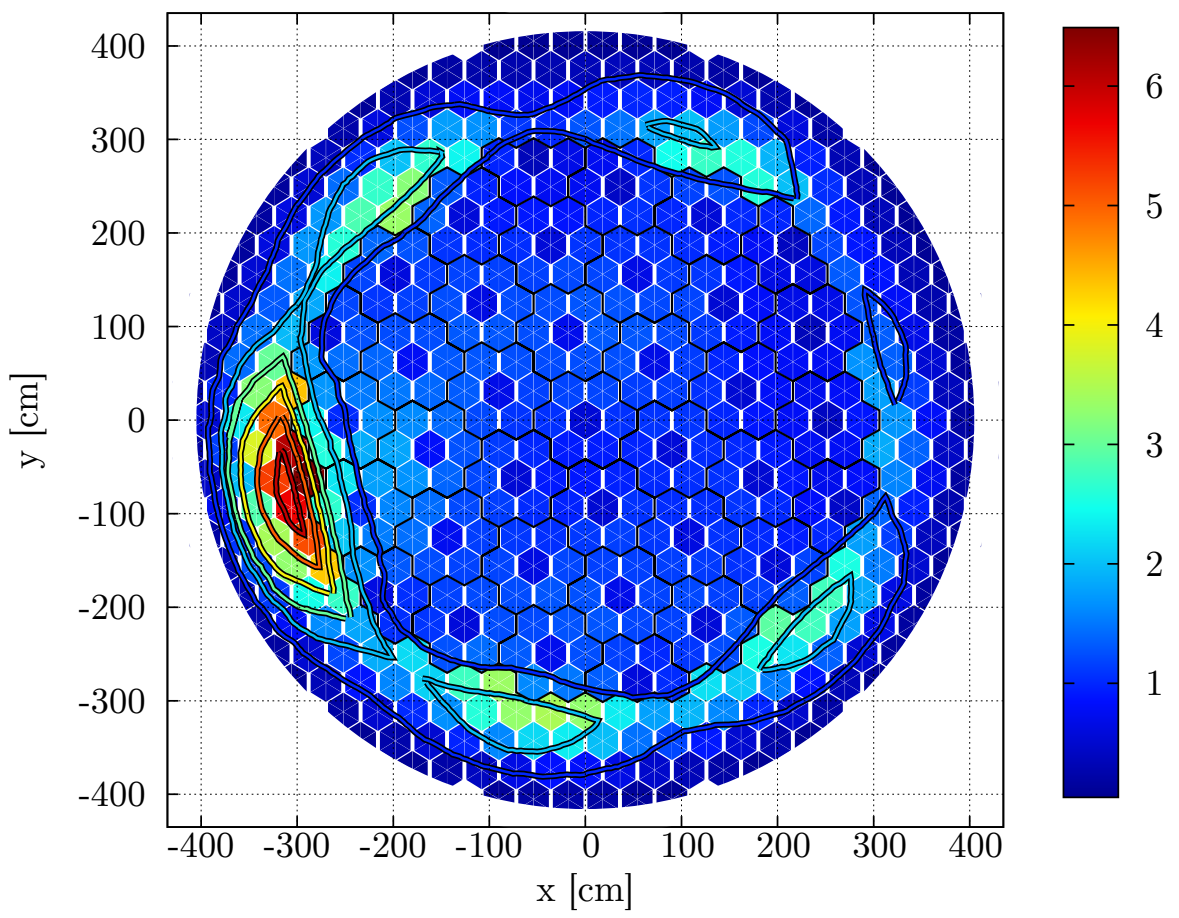

Figure 6.15: The OpenTRMM-calculated fundamental shape eigenfunction of the fullyrodded FSV reactor matches that calculated by General Atomics to help design the experiments. The colored lines designate contour lines matching the value of that given color. This normalized shape eigenfunction corresponds to the thermal energy interval, $g=4$. 
highest peak at the left edge of the core is adjacent to regions 30 and 31 . To obtain the best measured results of the fundamental $\alpha$ eigenvalue, detectors should be placed as close to these peaks as physically possible. These two regions have seven blocks each of blend 7/8 fuel: this is the fuel blend with the largest HEU-to-thorium ratio. Regions containing this fuel blend are at four other locations, causing the four other larger peaks in the fundamental shape eigenfunction. These peaks are smaller in magnitude because there are less blend $7 / 8$ fuel blocks at each of those other locations. The smooth contour lines of the homogeneous reactor mode expansion method are unable to measure the more discrete representation of the OpenTRMM shape eigenfunction.

The next few thermal shape eigenfunctions also agree with the calculated homogeneous reactor modes. Some of these shape eigenfunctions correspond to multiple eigenvalues, and all have positive and negative parts (Figure 6.16). The next two modes have one null contour line through the center of the core, the third and fourth

Mode 2

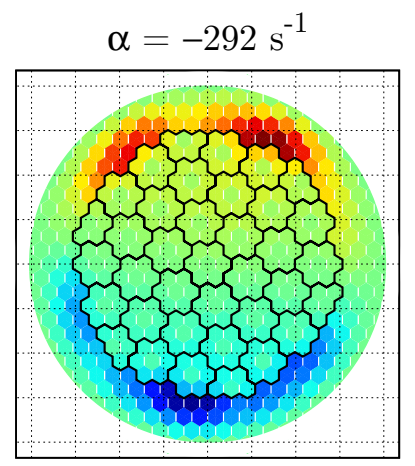

Mode 8

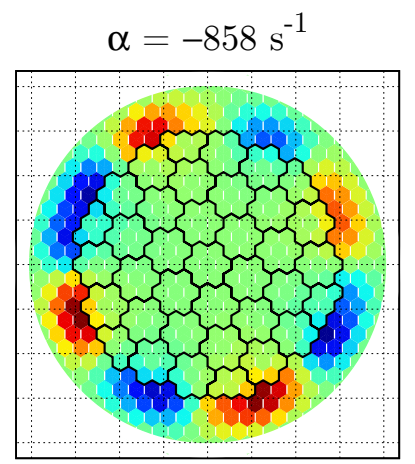

Mode 4

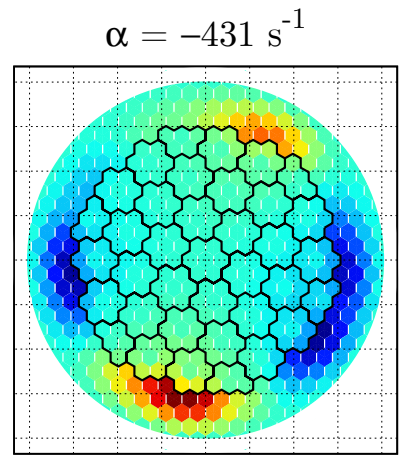

Mode 9

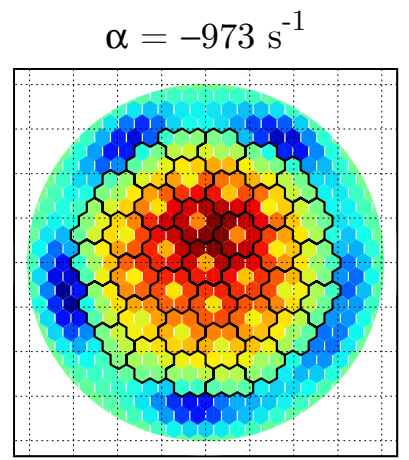

Mode 6

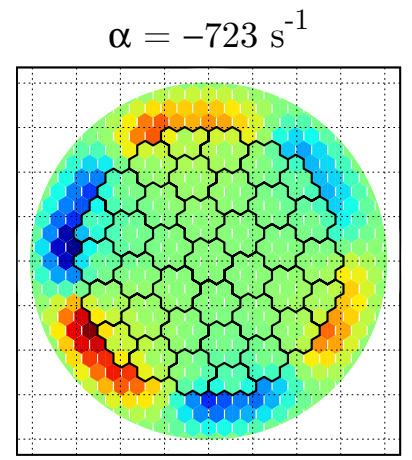

Mode 10

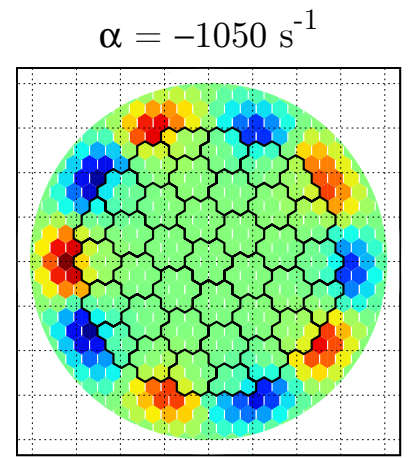

Figure 6.16: Selected OpenTRMM-calculated thermal shape eigenfunctions of the FSV reactor show the progression of the oscillatory shapes as $n$ increases. These normalized shape eigenfunctions correspond to the $g=4$ energy interval. 
higher eigenfunctions have two null contours, and this pattern repeats for many of the calculate $\alpha$ eigenvalues. Most of the thermal shape eigenfunctions are flat over the center of the core, with peaks coming at the core reflector interface: the ninth shape eigenfunction is the only one of the first few to be so sharply peaked in the center of the core. With increasing $n$, more peaks appear within the reflector.

While thermal shape eigenfunctions describe features within the reflectors, the fast shape eigenfunctions are nearly zero within the reflector, and vary within the core (Figure 6.17). The shape eigenfunctions progress similar to thermal shape eigenfunctions, except the features are isolated in the core instead of the reflector. The ninth shape eigenfunction is sharply peaked within the high HEU-to-thorium ratio regions adjacent to the central fuel region.

Application of the eigenfunction expansion with the available $\alpha$ eigenvalues and shape eigenfunctions gives the estimated detector response curve in any region within the position phase space. Examining three regions near the peak in the fundamental

Mode 1

$\alpha=-272 \mathrm{~s}^{-1}$

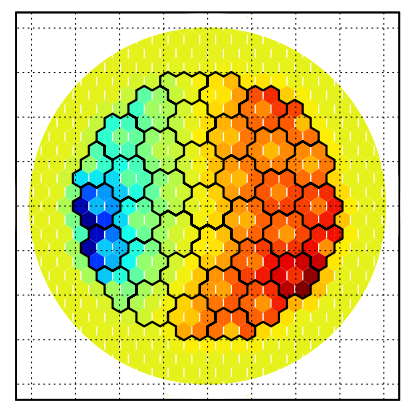

Mode 7

$\alpha=-849 \mathrm{~s}^{-1}$

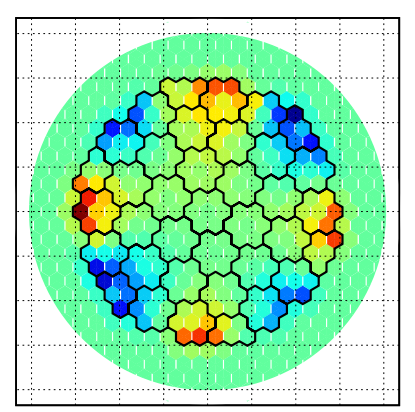

Mode 3

$\alpha=-425 \mathrm{~s}^{-1}$

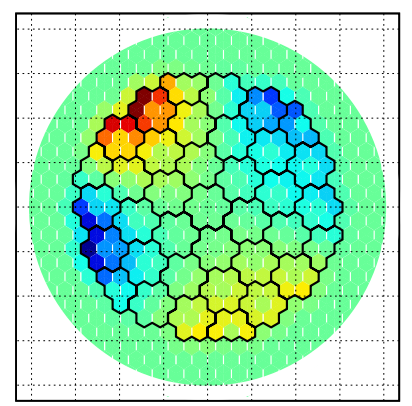

Mode 9

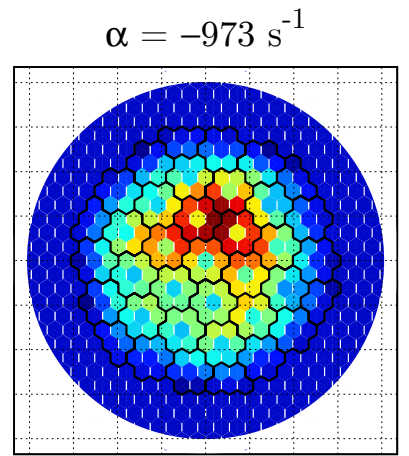

Mode 5

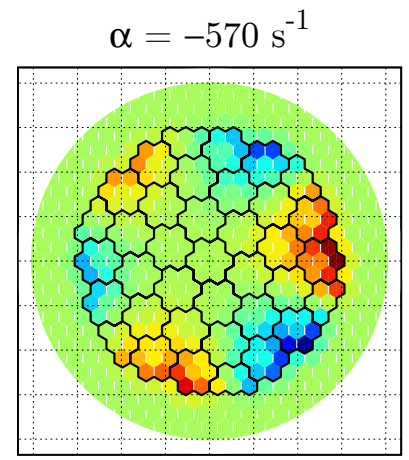

Mode 11

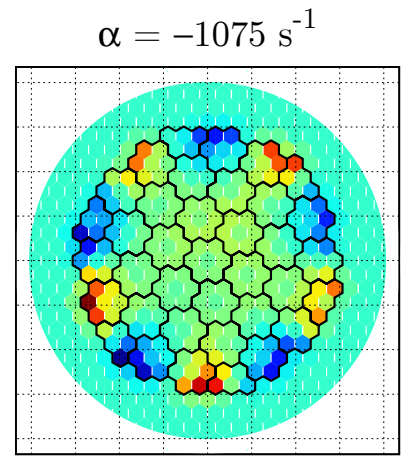

Figure 6.17: Selected OpenTRMM-calculated fast shape eigenfunctions of the FSV reactor show the progression of the oscillatory shapes as $n$ increases within the core. These normalized shape eigenfunctions correspond to the $g=1$ energy interval. 
shape eigenfunction shows the strong spatial dependence in FSV (Figure 6.18). While region 16 is adjacent to regions 30 and 31, it experiences a much more magnified response at a slightly earlier time. The detectors responses in regions 30 and 31 follow closely. While these calculated responses show the appropriate relative variation between the three detectors, OpenTRMM is unable to match the exact measured detector responses due to an insufficient number of position intervals defined in the TRM. Specifically, this is due to the lack of separate axial position intervals. With a single axial interval, all axial information about the time-dependence is lost, and the TRMM underestimates contributions to the expanded solution from higher kinetic modes. The discrepancy in the TRMM calculation is smaller if the sources and detectors were axially aligned during the experiments: the source was placed near the center of the core to minimize the contributions of higher kinetic modes, and the detectors were placed towards the top reflector to maximize the fundamental prompt kinetic mode. As defined in the TRMM, the sources and detectors are essentially aligned, so they closer to one another than in the actual experiment, resulting in the shorter time behavior in the calculated detector response.

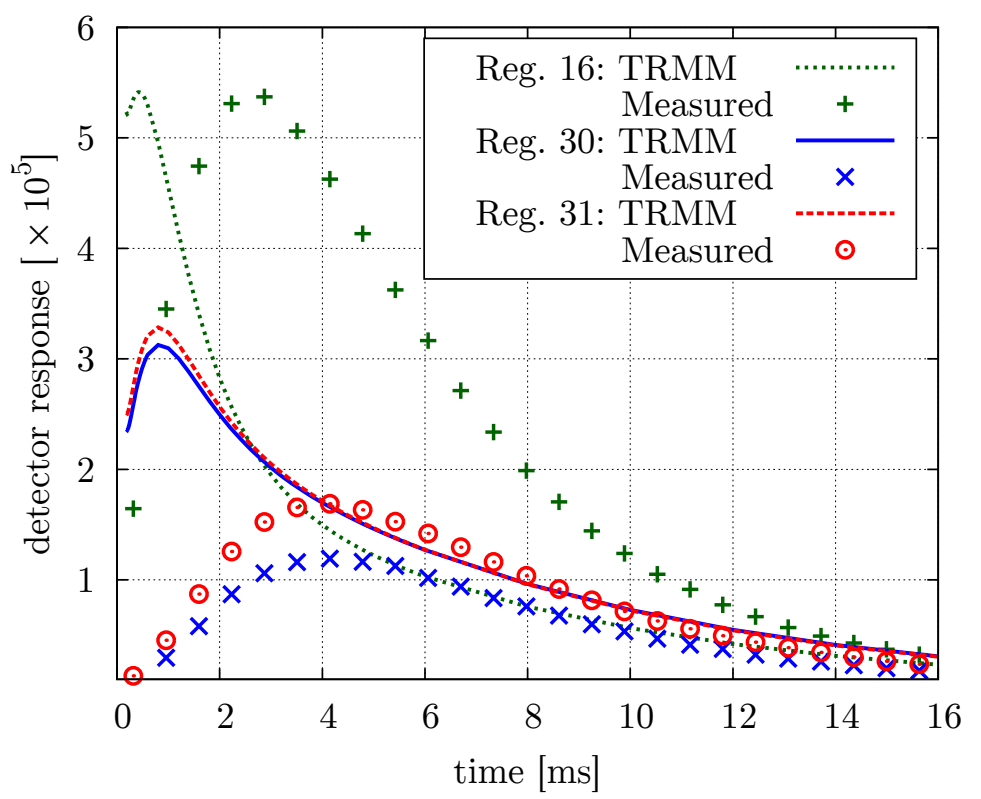

Figure 6.18: The OpenTRMM-calculated detector responses show the strong spatial dependence between the three adjacent regions selected to analyze the pulsed neutron experiments. The initial source defined in the OpenTRMM is in the position interval at the center of the core, is a pulse at $t=0$, and is in the highest energy interval $g=1$. With limited numbers of shape eigenfunctions, the solution before $0.1 \mathrm{~ms}$ is incorrect: the eigenfunction expansion uses only 499 kinetic modes. The measured data is from the FSV pulsed-neutron experiments. 


\subsection{Discussion}

The implementation of the TRMM into the OpenMC Monte Carlo code yields a method for calculating $\alpha$-eigenvalues and three-dimensional shape eigenfunctions of real systems, for which some measured data exists. Difficulties with this application to larger systems are mainly associated with finding an appropriate discretization of the large, multi-dimensional phase space. Accuracy of calculated eigenvalues is dependent on the chosen phase space and it is easy to eliminate certain higher shape eigenfunctions by using a coarse position phase space discretization.

Application to a homogeneous cube shows the stable calculation of the fundamental $\alpha$ eigenvalue and how certain higher shape eigenfunctions are negated by making simplifications in the phase space discretization. The ratios of the first few calculated prompt $\alpha$ eigenvalues follow the trend of the ratios obtained with one-speed diffusion theory. The first few shape eigenfunctions are also predicted by one-speed diffusion theory, though in some cases exhibit some slow convergence behavior for specific shape eigenfunctions. Some higher shape eigenfunctions are not adequately predicted by diffusion theory.

Application to the CALIBAN reactor shows the ability of OpenTRMM to obtain $\alpha$ eigenfunctions for this small fast system. While it is unable to accurately determine the prompt $\alpha$ eigenvalue, it meets expected ratios of the first few prompt eigenvalues from one-speed diffusion theory. An increase in the size of a fast burst reactor causes a reduction in the first few prompt eigenvalues. In this case, the effect of the next few prompt eigenfunctions is important to quantify during experiments.

Finally, application to the FSV high-temperature gas-cooled reactor shows the ability to obtain a fundamental $\alpha$ eigenvalue as well as a prompt eigenfunction that aligns with homogeneous mode calculations made by General Atomics. With a few number of OpenTRMM-calculated kinetic modes, the eigenfunction expansions are able to show the relative differences between detector responses within different regions of the reactor core. But, the overall detector responses do not match measured results because of the insufficient position phase space discretization: with a finer spatial mesh, these discrepancies decrease. Also, the TRMM underestimates the magnitude of the contributions from the higher eigenfunctions: this causes the next few higher kinetic modes to have a much lesser effect on the expanded solution than they should. 


\section{References}

[1] OpenMC Development Team, "The OpenMC Monte Carlo Code." Open Source, Website: mit-crpg.github.io/openmc, 2013.

[2] X-5 Monte Carlo Team, "MCNP - A General N-Particle Transport Code, Version 5, Volume I: Overview and Theory," Tech. Rep. LA-UR-03-1987, Los Alamos National Laboratory, 2003.

[3] R. B. Lehoucq, D. C. Sorensen, and C. Yang, ARPACK Users' Guide: Solution of Large Scale Eigenvalue Problems with Implicitly Restarted Arnoldi Methods. Philadelphia, Pennsylvania: Society for Industrial and Applied Mathematics, 1998.

[4] L. N. Trefethen and D. B. Bau III, Numerical Linear Algebra. Philadelphia, Pennsylvania: Society for Industrial and Applied Mathematics, 1997.

[5] B. Richard, "Neutron Noise Measurements on Fast Burst Reactor CALIBAN Determination of the Core Kinetic Parameters," Nuclear Science and Engineering. Accepted for Publication.

[6] S. E. Carney, F. B. Brown, B. C. Kiedrowski, and W. R. Martin, "Fission Matrix Capability for MCNP, Part II - Applications," Tech. Rep. LA-UR-13-20454, Los Alamos National Laboratory, 2013.

[7] N. Authier and B. Méchitoua, "Bare, Highly Enriched Uranium Fast Burst Reactor CALIBAN," in NEA/NSC/DOC/(95)03/II, vol. II, HEU-MET-FAST-080, ICSBEP, 2007.

[8] P. Humbert and B. Méchitoua, "Numerical Simulation of CALIBAN Reactivity Perturbation Experiments Using Neptunium-237 Samples," in Proc. Nuclear Mathematical and Computational Sciences: A Century in Review, A Century Anew, (Gatlinburg, Tennessee), April 2003.

[9] G. D. Spriggs, "Two Rossi- $\alpha$ Techniques for Measuring the Effective Delayed Neutron Fraction," Nuclear Science and Engineering, vol. 113, pp. 161-172, 1993.

[10] FSAR, "Fort St. Vrain Final Safety Analysis Report." General Atomic Report.

[11] B. R. Betzler, J. C. Lee, and W. R. Martin, "MCNP5 Analysis of Fort St. Vrain High-Temperature Gas-Cooled Reactor," in Trans. Am. Nucl. Soc., vol. 102, p. 515, 2010.

[12] F. W. Todt, "A Computer Program Package for the Fort St. Vrain Pulsed Neutron Measurements," Tech. Rep. Gulf-GA-B12354, Gulf General Atomic, 1973.

[13] W. Pfeiffer, "RTMODE and RZMODE - Two-Dimensional Computer Codes for Simulating Pulsed-Neutron Experiments," Tech. Rep. Gulf-GA-B10877, Gulf General Atomic, 1971. 


\section{CHAPTER 7}

\section{Summary and Future Work}

This thesis describes the theoretical formulation of the Transition Rate Matrix Method (TRMM) and its use to approximate the $\alpha$ eigenvalues and eigenfunctions of nuclear systems. It is dependent on the interpretation of operator of the adjoint $\alpha$ eigenvalue problem as the Transition Rate Matrix (TRM) describing the continuoustime Markov process that is neutron transport. The TRMM uses forward Monte Carlo tallies to obtain rates in a discrete TRM: in the limit of continuous positionenergy-direction intervals, this is equivalent to using adjoint Monte Carlo to obtain the rates. This thesis includes discussions of applications to infinite media and slabs via research Monte Carlo codes, and three-dimensional media via the OpenMC [1] Monte Carlo code.

Currently, methods of obtaining $\alpha$ eigenvalues and shape eigenfunctions are limited to diffusion- and transport-based matrix methods or $k$ - $\alpha$ iterative methods. In the former, deterministic formulations of a matrix equation yields discrete approximations of the $\alpha$ eigenvalues and shape eigenfunctions of the underlying problem: in most cases, these formulations are designed for a unique problem. In the latter, a criticality calculation is re-purposed to calculate the fundamental $\alpha$ eigenvalue of the problem, with additional subtraction methods necessary to obtain higher $\alpha$ eigenvalues. The TRMM is more similar to the matrix methods, except that it uses Monte Carlo methods to characterize the behavior of neutrons within a system: current matrix methods use only deterministic methods. Current Monte Carlo methods for calculating $\alpha$ eigenvalues are limited to the $k$ - $\alpha$ iteration and only yield the fundamental and potentially a few of the first higher $\alpha$ eigenvalues with an additional subtraction method. Thus, the TRMM provides a way for calculating higher $\alpha$ eigenvalues and shape eigenfunctions with Monte Carlo methods, and provides a way to predict the spatial variation of the neutron flux in short-time transients. 


\subsection{Calculated $\alpha$ Eigenvalues and Eigenfunctions}

In the initial implementation of the TRMM, the continuous-energy, infinite-medium, research Monte Carlo code TORTE accurately calculates the $k$ eigenvalue and TRM of hydrogenous and graphite media. Initial multigroup formulations provide analytical verification for the calculated $k$ and $\alpha$ eigenvalues. For continuous-energy media, the calculated eigenvalue spectrum approximates the true $\alpha$-eigenvalue spectrum. With an increasing number of energy intervals, TORTE calculations approach many of the discrete eigenvalues of the true $\alpha$ eigenvalue spectrum, where the highest calculated $\alpha$ eigenvalues are an approximation to the actual higher $\alpha$-eigenvalue spectrum. The TRMM calculates the appropriate fundamental $\alpha$ eigenvalue as predicted by the calculated $k$ eigenvalue of the system. The convergence of this fundamental $\alpha$ eigenvalue relates to the reactivity calculated from the collision estimate of the $k$ eigenvalue: the relative variation in the calculated fundamental $\alpha$ eigenvalue depends on how close the system is to critical. This correlation is because each collision event contributes tallies to the elements of the TRM and to the collision estimate of $k$. An increase in the number of energy intervals used to tally the TRM does not have an effect on the convergence rate of the prompt fundamental, next few prompt, and delayed $\alpha$ eigenvalues: the behavior neutrons within the entire system is more important to the calculation of these quantities that the behavior of neutrons within a single energy interval. But, the number of energy intervals does have an effect on the value of the calculated eigenvalues. Using an initial high-energy pulse source, the eigenfunction expansion and TDMC solutions match throughout transients in continuous-energy media. Noise observed in the eigenfunction expansion solution at early times suggest incompleteness of the set of calculated eigenfunctions: this behavior does not change with different numbers of energy intervals or neutron histories, but depends on the material of the medium and decays very quickly. These time-dependent solutions show that the fundamental mode is a poor approximation of the time-dependent flux for short times.

In the implementation of the TRMM to slabs, the continuous-energy, onedimensional, research Monte Carlo code TORTE-1D calculates the $k$ eigenvalue and TRM of hydrogenous and graphite media. Applications to one-speed slabs verify the TRMM calculation of the $\alpha$ eigenvalues with comparisons to the GFM [2]. For scattering and multiplying one-speed multi-region slabs, the TRMM accurately calculates the fundamental $\alpha$ eigenvalue, and most of the higher eigenvalues, with the only difficulties caused by eigenvalues closest to the Corngold limit. For pulse and 
constant sources, the eigenfunction expansion and TDMC solutions match, capturing the short-time dependence by calculating more eigenvalues than the discrete portion of the eigenvalue spectrum: it calculates complex eigenvalues within the continuum. The one-speed slabs also allow for the analysis of the position and direction interval choice in the absence of energy dependence. With an increase in the number of position or direction intervals, the calculated fundamental $\alpha$ eigenvalues approach those calculated by the GFM. Applications to continuous-energy slabs show the effect of discretizing a position-energy-direction phase space on the calculated $\alpha$ eigenvalues, and the effect of the calculated spectrum on the expanded flux solutions.

In the implementation of the TRMM to three-dimensional media, the OpenMC Monte Carlo code is modified to include the necessary tallies to obtain the TRM elements. While calculations converge to a fundamental $\alpha$ eigenvalue, difficulties with the large position-energy-direction phase space cause slower convergence of higher eigenvalues. For a homogeneous cube problem, the calculated $\alpha$ eigenvalues follow the general trend predicted by one-speed diffusion theory. Some of the asymmetric higher shape eigenfunctions converge only after a large number of neutron histories. This has little effect on the expanded flux solutions, which show the short-time dependence of the neutron flux. For the OpenMC model of the CALIBAN fast-burst reactor, the TRMM under-predicts the fundamental $\alpha$ eigenvalue [3] by about $10 \%$, but is able to calculate the proper $\beta_{\text {eff }}$ and the progression of the next few prompt $\alpha$ eigenvalues. Higher shape eigenfunctions also show the trend predicted by one-speed diffusion theory. For the OpenMC model of the FSV reactor, the TRMM calculates a reasonable estimate of the fundamental $\alpha$ eigenvalue (within $7 \%$ ) and several of the prompt shape eigenfunctions used in the expanded flux solutions. The prompt fundamental shape eigenfunction agrees with the homogeneous mode expansion method used by General Atomics to design the experiment [4]. The expanded flux solution shows the appropriate relative dependence of three detectors, but the TRMM underpredicts the contributions of the first few higher kinetic modes.

\subsection{Future Work}

In the formulations in this thesis, the delayed neutron precursor concentrations are discretized among the position phase space. This allows for the definition of precursor concentrations throughout the position phase space. Some variations on the treatment of these precursor concentrations and their effects on the calculated delayed $\alpha$ shape eigenfunctions are worth studying: this includes the reduction of the 
discretized precursor concentration phase space, and the inclusion of mobile fuel in the formulation. The former variation would simplify IRAM calculations by reducing the number of clustered delayed $\alpha$ eigenvalues. The latter variation requires an additional term in the precursor concentration equation and the assumption that the time-dependence of this term is separable from the remainder of the phase space in the formulation of the $\alpha$-eigenvalue problem. This has interesting connections to the theory of $\alpha$ modes and kinetics.

In calculating the TRM elements, the TRMM uses only sampled quantities. But, there are certain physical processes that are well known, i.e., neutron emission from fission is isotropic. If this is assumed, then only the energy emission spectrum is necessary to tally during the Monte Carlo random walk, and the probabilities may be distributed later over the appropriate direction intervals. This reduces memory requirements of the TRM tallies.

Little measured data of higher $\alpha$ eigenvalues exists, so there is not much to compare results to. The next highest $\alpha$ eigenvalues are important because they may help determine the point at which the position-energy-direction phase space discretization is sufficient for accurate calculations of eigenvalues. Also, finding some mathematical quantification of the statistical uncertainty of the higher eigenvalues is also of interest. This may be useful for the fission matrix method implementation in Monte Carlo, as both methods use tallied quantities to determine higher shape eigenfunctions.

Finally, there are a number of eigenvalue solvers applied to similar matrix methods for $\alpha$ eigenvalue calculations that may be of use when determining a larger portion of the spectrum of larger systems. As the discretized phase spaces become larger, the eigenvalues become more difficult to solve, but, a good amount is known about the

$\alpha$-eigenvalue spectrum. Always, the more calculated shape eigenfunctions that may be extracted form the TRM, the better.

\section{References}

[1] OpenMC Development Team, "The OpenMC Monte Carlo Code." Open Source, Website: mit-crpg.github.io/openmc, 2013.

[2] D. E. Kornreich and D. K. Parsons, "Time-eigenvalue Calculations in Multi-region Cartesian Geometry Using Green's Functions," Annals of Nuclear Energy, vol. 32, pp. 964-985, 2005.

[3] B. Richard, "Neutron Noise Measurements on Fast Burst Reactor CALIBAN Determination of the Core Kinetic Parameters," Nuclear Science and Engineering. Accepted for Publication. 
[4] W. Pfeiffer, "RTMODE and RZMODE - Two-Dimensional Computer Codes for Simulating Pulsed-Neutron Experiments," Tech. Rep. Gulf-GA-B10877, Gulf General Atomic, 1971. 